\title{
Haptic Foot Interactions on a 4-link Planar Rehabilitation Robot
}

by

\section{Kyle Klumper}

\author{
A Thesis submitted to \\ the Faculty of Graduate Studies and Research \\ in partial fulfilment of \\ the requirements for the degree of \\ Master of Applied Science
}

\author{
Ottawa-Carleton Institute for \\ Mechanical and Aerospace Engineering \\ Department of Mechanical and Aerospace Engineering \\ Carleton University \\ Ottawa, Ontario, Canada
}

July 30, 2012

Copyright (C)

2012 - Kyle Klumper 
Library and Archives

Canada

Published Heritage

Branch

395 Wellington Street

Ottawa ON K1A ON4

Canada
Bibliothèque et

Archives Canada

Direction du

Patrimoine de l'édition

395 , rue Wellington

Ottawa ON K1A ON4

Canada
Your file Votre référence

ISBN: 978-0-494-93521-7

Our file Notre référence

ISBN: $978-0-494-93521-7$
NOTICE:

The author has granted a nonexclusive license allowing Library and Archives Canada to reproduce, publish, archive, preserve, conserve, communicate to the public by telecommunication or on the Internet, loan, distrbute and sell theses worldwide, for commercial or noncommercial purposes, in microform, paper, electronic and/or any other formats.

The author retains copyright ownership and moral rights in this thesis. Neither the thesis nor substantial extracts from it may be printed or otherwise reproduced without the author's permission.
AVIS:

L'auteur a accordé une licence non exclusive permettant à la Bibliothèque et Archives Canada de reproduire, publier, archiver, sauvegarder, conserver, transmettre au public par télécommunication ou par l'Internet, prêter, distribuer et vendre des thèses partout dans le monde, à des fins commerciales ou autres, sur support microforme, papier, électronique et/ou autres formats.

L'auteur conserve la propriété du droit d'auteur et des droits moraux qui protege cette thèse. $\mathrm{Ni}$ la thèse ni des extraits substantiels de celle-ci ne doivent être imprimés ou autrement reproduits sans son autorisation.
In compliance with the Canadian Privacy Act some supporting forms may have been removed from this thesis.

While these forms may be included in the document page count, their removal does not represent any loss of content from the thesis.
Conformément à la loi canadienne sur la protection de la vie privée, quelques formulaires secondaires ont été enlevés de cette thèse.

Bien que ces formulaires aient inclus dans la pagination, il n'y aura aucun contenu manquant. 


\section{Abstract}

Preliminary development of the Virtual Gait Rehabilitation Robot (ViGRR) has realized the concept platform into a 4-link planar robot, which has been verified through force controlled experiments involving a user's hand.

This thesis presents the further development of ViGRR into a lower extremity haptic rehabilitation platform. Hardware modifications of the ViGRR platform, which are necessary for human experiments are assessed and completed. These modifications include a patient seat, base platform, electronic interface board, updated kinematic calibration, more reliable limit switches, and a magnetically detachable foot plate for safe human-robot interaction. This thesis then describes the development, implementation, and verification of a real-time compatible 6-DoF haptic rendering technique, which will be used for the subsequent experiments. Experiments are then conducted with a human subject in order to assess the ability of ViGRR to reproduce the swing and stance phase of a gait cycle normally seen through walking. The results from the stance phase experiments prove that a scaled version of the vertical ground reaction force can be produced. The swing phase experiments, however, show that the impedance parameters of the current control scheme prevent the subject from attaining a smooth tracking of a gait trajectory. Finally, a set of virtual reality exercises are designed and evaluated in order to highlight the capabilities of ViGRR with respect to lower extremity physical rehabilitation. 
To my family and friends, whose endless support made this thesis possible. 


\section{Table of Contents}

$\begin{array}{lll}\text { Abstract } & \text { ii }\end{array}$

Table of Contents $\quad$ iv

List of Tables $\quad$ viii

$\begin{array}{ll}\text { List of Figures } & \text { ix }\end{array}$

1 Introduction $\quad 1$

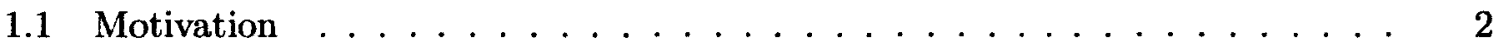

1.2 Approaches to Haptic Rehabilitation Robotics . . . . . . . . . . . . 3

1.2.1 Upper Extremity Haptic Rehabilitation . . . . . . . . . . . . 4

1.2.2 Lower Extremity Haptic Rehabilitation . . . . . . . . . . . . 10

1.3 Platform Introduction $\ldots \ldots \ldots \ldots \ldots \ldots$

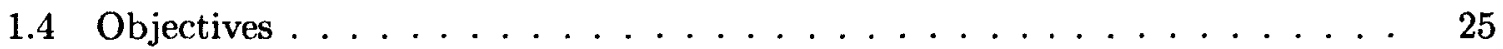

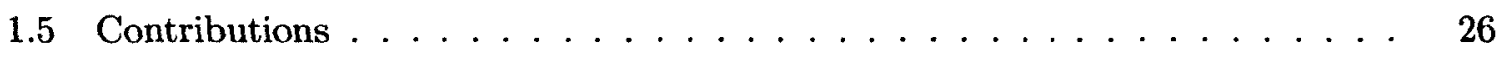

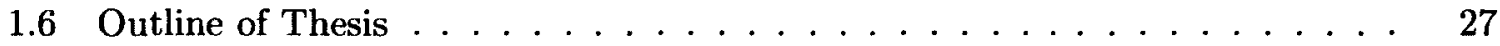

2 Virtual Gait Rehabilitation Robot (ViGRR) 28

2.1 ViGRR Introduction $\ldots \ldots \ldots \ldots \ldots$

2.1 .1 Concept . . . . . . . . . . . . . . . . 28

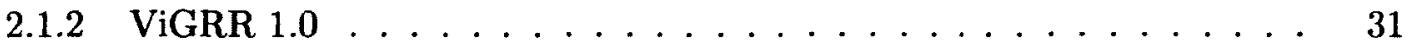

2.1.3 Required Design Enhancements for ViGRR $1.0 \ldots \ldots 36$

2.2 Base Platform . . . . . . . . . . . . . . . . . . . . . 38 
2.3 Patient Seat . . . . . . . . . . . . . . . . 38

2.4 Hard Limit Switches . . . . . . . . . . . . . . . . 38

2.5 Interface Board . . . . . . . . . . . . . . . 41

2.6 Kinematic Calibration . . . . . . . . . . . . . . . 43

2.6.1 Coordinate Frames and the Optotrak Motion Capture System . . . . 43

2.6 .2 Link Length Estimation . . . . . . . . . . . . . . . . 46

2.7 Foot Plate Design . . . . . . . . . . . . . . . . . . 48

2.7 .1 Design Requirements . . . . . . . . . . . . . . 48

2.7.2 Initial Foot Plate Design . . . . . . . . . . . . . . . 49

2.7.3 Modified Foot Plate Design . . . . . . . . . . . . . . . 52

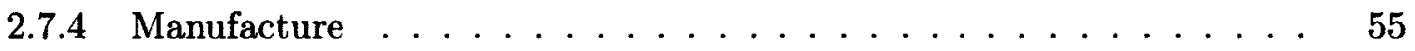

2.7 .5 Verification ...................... 56

2.7 .6 Discussion . . . . . . . . . . . . . . . . 60

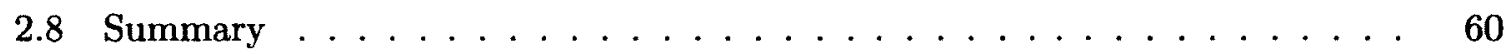

3 Haptic Delivery with ViGRR $\quad 61$

3.1 Admittance Control vs. Impedance Control . . . . . . . . . . . . 61

3.1 .1 ViGRR Admittance Control . . . . . . . . . . . . . . . . 63

3.2 Haptics Theory . . . . . . . . . . . . . . . . . . 65

3.2 .1 Collision Detection . . . . . . . . . . . . . 66

3.2.2 Force Computation . . . . . . . . . . . . . 67

3.2.3 Direct Rendering vs. Virtual Coupling . . . . . . . . . . 67

3.3 Haptic Rendering Requirements . . . . . . . . . . . . . . . . 69

3.4 Haptic Rendering Algorithm . . . . . . . . . . . . . 71

3.4.1 Open Dynamics Engine . . . . . . . . . . . . . 71

3.4 .2 The Algorithm ........................ 75

3.5 Haptic Rendering Implementation $\ldots \ldots \ldots \ldots$

3.6 Haptic Rendering Verification . . . . . . . . . . . . . . 79

3.6.1 Effects of Varying $K$ and $D \ldots \ldots \ldots \ldots \ldots \ldots$ 
3.6 .2 Friction Test ......................... 81

3.6 .3 Box Test $\ldots \ldots \ldots \ldots \ldots \ldots$

3.7 Haptic Device Performance Measures . . . . . . . . . . . . . 85

3.7.1 Degrees of Freedom and Classification $\ldots \ldots \ldots \ldots$

3.7.2 Device-Body Interface . . . . . . . . . . . . . 86

3.7 .3 Range of Motion . . . . . . . . . . . . . . . 86

3.7.4 Manipulability Ellipsoid . . . . . . . . . . . . . . . 88

3.7 .5 Mass and Damping . . . . . . . . . . . . . . . . . 91

3.7 .6 Resolution . . . . . . . . . . . . . . . . . 94

3.7.7 Precision and Repeatability . . . . . . . . . . . . . 96

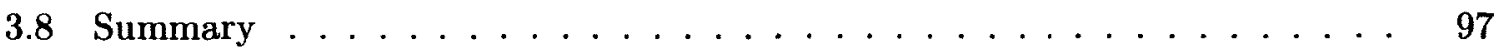

4 Lower Limb Haptic Rehabilitation with ViGRR 98

4.1 Gait Fundamentals . . . . . . . . . . . . . . . . . . . 99

4.1.1 Gait Cycle . . . . . . . . . . . . . . . . . . 99

4.1 .2 Ground Reaction Forces . . . . . . . . . . . . . . . 100

4.2 Gait Experiments . . . . . . . . . . . . . . . . . . . . . . . 104

4.2.1 Swing Phase Experiments . . . . . . . . . . . . 105

4.2.2 Stance Phase Experiments . . . . . . . . . . . . . . . . . . . . . . . . 119

4.2 .3 Virtual Tool Summary . . . . . . . . . . . . . . . 128

4.2.4 Stance Phase General Discussion . . . . . . . . . . . . . . . 129

4.3 Virtual Reality Exercises . . . . . . . . . . . . . . . . . 130

4.3.1 Exercise 1: Leg Press . . . . . . . . . . . . . . 132

4.3.2 Exercise 2: Brickbreaker . . . . . . . . . . . . . 134

4.3 .3 Exercise $3:$ Maze . . . . . . . . . . . . . . . . . 137

4.3.4 Exercise 4: Ball Balance . . . . . . . . . . . . . 139

4.3 .5 Summary ........................... 143

5 Conclusions and Future Work $\quad 145$

5.1 Conclusions . . . . . . . . . . . . . . . . 145 


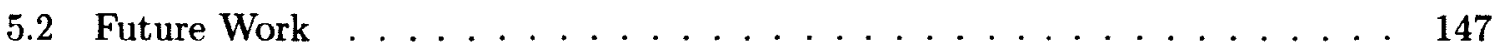

$\begin{array}{ll}\text { List of References } & 150\end{array}$

$\begin{array}{ll}\text { Appendix A Ethics Clearance Form } & 155\end{array}$

$\begin{array}{ll}\text { Appendix B Interface Board Diagrams } & 156\end{array}$

$\begin{array}{ll}\text { Appendix C Foot-Robot Coupling } & 158\end{array}$

C.1 Contact Parameters . . . . . . . . . . . . . . . . 158

C.2 Robot Flexibility . . . . . . . . . . . . . . . . . . 159 


\section{List of Tables}

2.1 ViGRR Drive Components. . . . . . . . . . . . . . . . . 32

2.2 Danaher Motor Specifications. . . . . . . . . . . . . . . . 32

2.3 Harmonic Drive Specifications. . . . . . . . . . . . . . . 33

2.4 Force Torque Sensor Specifications. . . . . . . . . . . . . . . 33

2.5 Safety Features for ViGRR 1.0. . . . . . . . . . . 35

2.6 Base Platform Maximum Loading Condition for FEA. . . . . . . . . . . 38

2.7 Link Parameters from Kinematic Calibration. . . . . . . . . . . . . . . . 47

3.1 Mass and Damping Parameters of ViGRR in Admittance Control for Hand Coupling . . . . . . . . . . . . . . . . . . 94

3.2 ViGRR Resolution $\ldots \ldots \ldots \ldots \ldots$

3.3 Repeatability Range for 4 End-Effector Positions . . . . . . . . . . . 97

4.1 Patient Dimensions. . . . . . . . . . . . . . . . 105

4.2 Settings for the Swing Phase Experiments. . . . . . . . . . . . 107

4.3 Maximum Tracking Errors for a Gait Trajectory. . . . . . . . . . . . . 113

4.4 Maximum Interaction Loads for a Gait Trajectory. . . . . . . . . . . 115

4.5 Settings for the Swing Phase Experiments. . . . . . . . . . . . . . . 122

4.6 Leg Press Exercise Summary. . . . . . . . . . . . . . . . . . . . 134

4.7 Brickbreaker Exercise Summary. . . . . . . . . . . . . . . . . . 137

4.8 Maze Exercise Summary. . . . . . . . . . . . . . . . . . . . . . . 139

4.9 Ball Balance Exercise Summary. . . . . . . . . . . . . . . . . . 143 


\section{List of Figures}

1.1 The HapticKnob $[1] \ldots \ldots \ldots \ldots \ldots$

1.2 The Quanser Haptic Rehabilitation Robot [2] . . . . . . . . . . . . 5

1.3 The PHANTOM OMNI Haptic Device $[3] \ldots \ldots \ldots$

1.4 The PHANTOM haptic device combined with Crystal Eyes CE-2 setup [4] 7

1.5 The Driver's SEAT haptic device $[5] \ldots \ldots \ldots \ldots$

1.6 The Rutgers Master II prototype $[6] \ldots \ldots \ldots \ldots$

1.7 The CyberGrasp ${ }^{T M}$ haptic interface $[7] \ldots \ldots \ldots$

1.8 The HapticWalker $[8] \ldots \ldots \ldots \ldots \ldots \ldots$

1.9 Motion tracking data for the Treadmill mode foot trajectory and CoM mode foot trajectory $[9] \ldots \ldots \ldots \ldots \ldots$

1.10 Vertical and horizontal forces acting on a users foot. $F_{v e r t}$ (Dashed-dotted black) and $F_{\text {horiz }}$ (solid blue). User body weight is indicated by the red

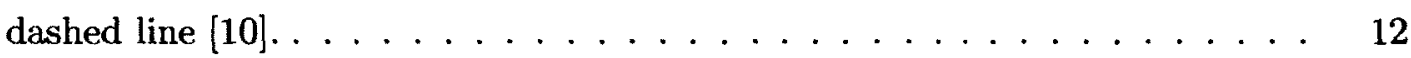

1.11 The Rutgers Ankle haptic platform [11] . . . . . . . . . . . . . . 13

1.12 Two Rutgers Ankles in a rehabilitation setup [12] . . . . . . . . . . . . 14

1.13 The Rutgers Mega Ankle foot plates [13] . . . . . . . . . . . . 15

1.14 The Rutgers Mega Ankle Virtual Crosswalk [14] . . . . . . . . . . . . 15

1.15 A CAD model of the CDLI $[15] \ldots \ldots \ldots \ldots$

1.16 CAD model of the $6-$ DoF rehabilitation robot $[16] \ldots \ldots \ldots$

1.17 The Gait Master prototype [17] . . . . . . . . . . . . . . . . 19

1.18 Pressure profiles of GM and real stair data (a) climbing up stairs (b) climbing down stairs $[17] \ldots \ldots \ldots \ldots \ldots \ldots \ldots \ldots$ 
1.19 Exoskeletal type rehabilitation robots $\ldots \ldots \ldots 22$

1.20 Concept CAD model of the ViGRR platform . . . . . . . . . . . 24

2.1 Block Diagram of the ViGRR platform. . . . . . . . . . . . . . 29

2.2 Concept CAD model of the ViGRR platform. . . . . . . . . . . 30

2.3 ViGRR 1.0 prototype. . . . . . . . . . . . . . . . . . . 31

2.4 The ATI Industrial Automation 6 axis force/torque sensor mounted on the ViGRR end-effector. . . . . . . . . . . . . . . . . 33

2.5 ViGRR interface block diagram. . . . . . . . . . . . . 36

2.6 FEA analysis of the base platform. . . . . . . . . . . . . . 39

2.7 Side view of the patient seat. . . . . . . . . . . . . 39

2.8 D-F9P solid state proximity sensor from SMC . . . . . . . . . . . . 40

2.9 Limit switch configuration on ViGRR. The yellow blocks represent the soft limits and black blocks represent the hard limits. . . . . . . . . . 40

2.10 Interface Board PCB. . . . . . . . . . . . . . . . . 41

2.11 Optotrak Certus Motion Capture System and rigid body marker by NDI. . 44

2.12 Coordinate frames of ViGRR. . . . . . . . . . . . . . . . 45

2.13 Optotrak markers placed on ViGRR. $\{O F P\}$ represents the marker frame located on the footplate and $\{b\}$ represents the marker frame located on the base platform. . . . . . . . . . . . . . . . 46

2.14 Coordinate system of the previous ViGRR end-effector. . . . . . . . . . 49

2.15 Initial design of the foot plate. 1) FT sensor. 2) Electromagnet. 3) Extension Spring. 4) Contraction Spring. 5) Foot attachment plate. 6) Guide rods. 7) Pretension screws. . . . . . . . . . . . . . . . 50

2.16 Schematic diagram of the electromagnet circuit where MCL-24 represents the electromagnet. ............................... 51

2.17 (a) Initial end-effector orientation. (b) Modified end-effector orientation. (FT

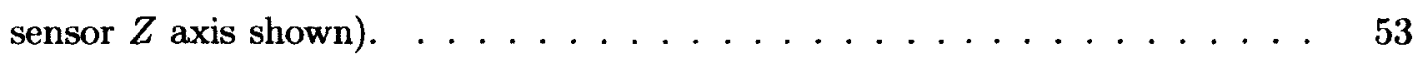

2.18 CAD model of the end-effector with magnet engaged (a) and disengaged (b). 54

2.19 Exploded view of the end-effector illustrating the locking cone mechanism. . 55 
2.20 Manufactured end-effector with magnet engaged (a) and disengaged (b). . . 56

2.21 The 3 main decoupling conditions. . . . . . . . . . . . . . 57

2.22 Break off moment about the $X$ axis for the bottom magnet. . . . . . . 58

2.23 Break off moment about the $Y$ axis for the bottom magnet. . . . . . . 59

2.24 Break off moment about the $Z$ axis for the bottom magnet. . . . . . . . 59

3.1 Block Diagram of the impedance control scheme. . . . . . . . . . . 62

3.2 Block Diagram of the admittance control scheme. . . . . . . . . . . 63

3.3 Block Diagram of the ViGRR control scheme. . . . . . . . . . 65

3.4 Block diagram of direct rendering. . . . . . . . . . . . . . . 68

3.5 Block diagram of virtual coupling. . . . . . . . . . . . . . . . 69

3.6 Example of virtual coupling. The wire frame spoon is the position and orientation of the haptic device and the solid spoon is the closest possible solution $[18] \ldots \ldots \ldots \ldots \ldots \ldots \ldots \ldots \ldots \ldots \ldots \ldots \ldots \ldots \ldots \ldots \ldots \ldots$

3.7 Graphical representation of the friction cone. $\vec{N}$ is the normal force, $\vec{T}$ is the tangential force and $\vec{R}$ is the resultant of the two [19]. . . . . . . . .

3.8 Virtual world showing the haptic device (red box) and the virtual tool (blue

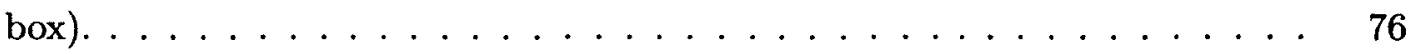

3.9 Block diagram of the haptic controller. . . . . . . . . . . . . . 77

3.10 Block diagram of the parallel processes of ViGRR . . . . . . . . . . 79

3.11 Visualization of the experimental setup to test varying $K$ and $D$ with the haptic rendering algorithm. .................. 80

3.12 Position and force associated with a virtual wall contact in the $X$ direction for varying contact parameters. . . . . . . . . . . . . . 81

3.13 Visualization of the experimental setup to test friction with the haptic rendering algorithm. . . . . . . . . . . . . . 82

3.14 Measured and desired forces and torques for a virtual wall contact test with

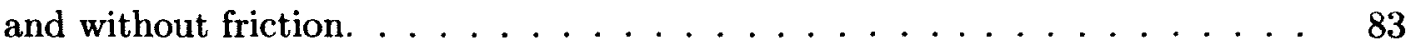

3.15 Haptic rendering box test for reference with Figure 3.16 . . . . . . . . . 84 
3.16 Haptic device and virtual tool positions with respect to time. The virtual tool is defined as a box. . . . . . . . . . . . . .

3.17 The device-body interface of the ViGRR. The user's foot is strapped to the footplate by two velcro strips. . . . . . . . . . . . . .

3.18 Reachable workspace of ViGRR for end-effector angle $\phi=0^{\circ}$. The base of the robot is defined as $X=Y=0 m$ and any point behind $0.3 \mathrm{~m}$ is not shown as it is not considered the normal operating range of ViGRR. . . . . . . 88

3.19 Velocity manipulability ellipsoids for ViGRR at different joint configurations. 89

3.20 Global manipulability measure within the ViGRR workspace for an endeffector angle $\phi=0^{\circ} \ldots \ldots \ldots \ldots \ldots$. . . . . . . . . . . 90

3.21 Force and velocity manipulability ellipses for a single configuration of ViGRR 91

3.22 Circle trajectory for admittance controlled experiments. The yellow ball represents the trajectory to be followed and the red ball represents the virtual tool of the device. . . . . . . . . . . . . . . .

3.23 Forces in the $X$ and $Y$ directions while following a circle trajectory of $r=$ $0.1 \mathrm{~m}$ and $T=2.5 \mathrm{~s}$.

3.24 Moment in the $\phi$ direction while following a rotation trajectory of $45^{\circ}$ and $T=2.5 s$

3.25 Task space resolution of ViGRR in $X$ and $Y$ directions depending on the position of the end-effector.

4.1 Position of the right leg (grey) during a gait cycle [20] . . . . . . . . 100

4.2 Single and double support timing during a gait cycle [20]. . . . . . . . . 101

4.3 Ground reaction forces in the lateral, foreaft and vertical directions for right foot (solid line) and left foot (dashed line) [20]. . . . . . . . . . . . 102

4.4 Ground reaction force vertical direction with a heelstrike transient [20]. . . 103

4.5 Pathway of the foot centre of pressure in normal gait [21] . . . . . . . . 104

4.6 Reference gait trajectory based on patient dimensions. . . . . . . . . . . 106

4.7 Experimental setup of the swing phase trajectory tracking experiments. . . 107 
4.8 Desired and measured gait trajectories during two swing phase trials for a cadence of (a) $7.5 \frac{\mathrm{steps}}{\mathrm{min}}$ and (b) $12.5 \frac{\mathrm{steps}}{\mathrm{min}} \ldots \ldots \ldots \ldots \ldots$

4.9 Position errors during one trial of the swing phase trajectory experiment for

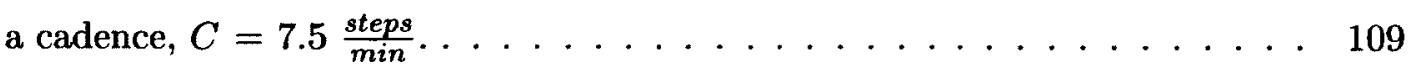

4.10 Position errors during one trial of the swing phase trajectory experiment for a cadence, $C=12.5 \frac{\text { steps }}{\text { min }} \ldots \ldots \ldots \ldots \ldots \ldots$

4.11 Interaction loads during one trial of the swing phase trajectory experiment for a cadence, $C=7.5 \frac{\text { steps }}{\min } . F_{x}, F_{y}$, and $M_{\phi}$ represent the forces in the $X$ and $Y$ directions and the moment about the $\phi$ axis respectively. . . . . . .

4.12 Interaction loads during one trial of the swing phase trajectory experiment for a cadence, $C=7.5 \frac{\text { steps }}{\min } . F_{x}, F_{y}$, and $M_{\phi}$ represent the forces in the $X$ and $Y$ directions and the moment about the $\phi$ axis respectively. . . . . . .

4.13 Force and torque measurements during free motion experiments for the HapticWalker $[10] . \ldots \ldots \ldots \ldots \ldots \ldots \ldots \ldots \ldots \ldots$

4.14 Device-Body Coupling of ViGRR for the hand and foot. . . . . . . . . 116

4.15 Gravity compensation admittance controller. . . . . . . . . . . . . 117

4.16 Simple gravity compensation. . . . . . . . . . . . . . . . . . 118

4.17 Virtual tool profiles for producing ground reaction forces (a) Rectangular (b) Cirular (c) Hybrid. . . . . . . . . . . . . . . . . . . . 120

4.18 Experimental setup of the stance phase trajectory tracking experiments. . . 121

4.19 Ground Reaction Forces and $\mathrm{CoP}$ for a rectangular virtual tool. Sections A, $\mathrm{B}$, and $\mathrm{C}$ represent heel down, mid-stance, and toe off respectively. $F_{m}$ and $F_{d}$ represent the measured forces at the foot plate and desired forces from the haptic rendering algorithm. . . . . . . . . . . . .

4.20 Ground Reaction Forces and CoP for a circular virtual tool. $F_{m}$ and $F_{d}$ represent the measured forces at the foot plate and desired forces from the haptic rendering algorithm. . . . . . . . . . . . . . 125 
4.21 Ground Reaction Forces and CoP for a hybrid virtual tool. $F_{m}$ and $F_{d}$ represent the measured forces at the foot plate and desired forces from the haptic rendering algorithm. . . . . . . . . . . . . . 127

4.22 Suggested extra rotational degree of freedom about the $Y$ axis. . . . . . . . 130

4.23 Leg press exercise with ViGRR. (a) The virtual tool and weight are shown as the red and grey boxes respectively. (b) The patient pushes in the $X$ direction, acting against gravity.

4.24 Leg Press experiment for a $5 \mathrm{~kg}$ and $10 \mathrm{~kg}$ weight. The blue line represents the measured forces at the FT sensor $\left(F_{m}\right)$ and the red line represents the haptic interaction forces $\left(F_{d}\right) \ldots \ldots \ldots \ldots \ldots$

4.25 Brickbreaker exercise . . . . . . . . . . . . . . . . 135

4.26 Subject interaction loads during the brickbreaker exercise. . . . . . . . . . 136

4.27 Maze exercise for ViGRR. (a) Virtual tool in free space. (b) Virtual tool in contact with walls.

4.28 Position of the end-effector during the maze exercise. The red lines represent the maze walls and the blue line represents the end-effector position. . . . . 138

4.29 Balance training exercise for ViGRR. (a) The balance block starts large for severely impaired patients. (b) Smaller balance block for increased difficulty. 140

4.30 Force and paddle angle for the ball balance exercise with $g=1 \frac{m}{s^{2}} \ldots \ldots \ldots 141$

4.31 Force and paddle angle for the ball balance exercise with $g=1 \frac{m}{s^{2}} \ldots \ldots$. . 142

A.1 Carleton University Ethics Clearance Form . . . . . . . . . . . . 155

B.1 Interface board schematic diagram $\ldots \ldots \ldots \ldots$

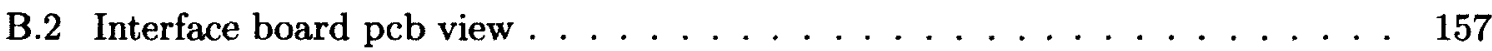

C.1 Haptic interaction force for a subject following a gait trajectory into a virtual ground. The red line show the desired haptic force $F_{d}$ and the blue line represents the measured force $F_{m} \ldots \ldots \ldots \ldots \ldots$

C.2 FFT of the oscillatory haptic interaction forces with varying stiffness and damping parameters. The frequency spike seen in each plot corresponds to $6.65 \mathrm{H}$ 
C.3 $Z$ Deflection at the end-effector of the robot and corresponding FFT from a force impulse. . . . . . . . . . . . . . . . 160

C.4 Steel link replacement for ViGRR link 1. . . . . . . . . . . . 161

C.5 $Z$ Deflection at the end-effector of the robot and corresponding FFT from a force impulse with a steel first link. . . . . . . . . . . . . . . 162 


\section{Chapter 1}

\section{Introduction}

According to the World Health Organization, the world's elderly population - people 60 years of age and older - is the fastest growing age group [22]. With an increase in the elderly population comes an increase in age related disorders such as multiple sclerosis, spinal cord injuries, parkinson's disease and stroke. On top of that, stroke costs the Canadian economy $\$ 3.6$ billion a year in physician services, hospital costs, lost wages, and decreased productivity [23]. From these statistics, it is clear there is a growing demand for not only conventional rehabilitation, but an investigation into alternative methods such as robotics.

Assistive robotic technology has been used as a means to aid individuals in their daily lives, however there is an apparent need of robotic devices which will go beyond that in order to facilitate their recovery [24]. Due to the nature of physical therapy, these devices will not only assist the patient in the rehabilitation process but will also reduce the physical strain on the occupational therapist and increase their patient throughput. Although many people share a common fear that the robots are meant to replace therapists, it must be stated that they are only meant as a tool to increase the intensity of therapy while adhering to motor rehabilitation practices [25].

Hemiplegia is one of the most common impairments after stroke and can lead to a significant reduction in gait performance [26]. Therefore, gait rehabilitation is of particular interest to patients who have suffered a central nervous system (CNS) disorder. This thesis 
will outline the continuing development of a new end-effector based gait rehabilitation robot. In particular, these developments are designed to allow for relevant biomedical experiments to be conducted with the human foot.

\subsection{Motivation}

Over the past decade, robots have shown promise as a tool used in the rehabilitation process $[25,27]$. Although there are a plethora of advantages to using robots for rehabilitation, few studies have shown that there is a significant improvement to using robots over conventional therapy. Due to the lack of supporting data, it is important to continue this research in order to develop a better understanding of the advantages and limitations of rehabilitation robotics.

\section{Repetition and Intensity}

Robots are, by nature, designed to carry out repetitive tasks. A robot can relieve the therapist from the burden associated with high levels of physical exertion. This relief would prevent the therapist from developing long term health problems associated with repetitive and intensive tasks [28].

\section{Patient Monitoring}

Another benefit of robots in the rehabilitation process is the ability for quantitative patient monitoring. Detailed information on joint kinematics, EMG data and interaction forces can be recorded for future processing. This data is necessary to determine the quality of a patient's therapy, as well as the patient's overall progress. Continuous assessment of patient data can also be tracked without the need of a therapist present which introduces new techniques such as home therapy [29].

\section{Virtual Reality and Immersion}

Virtual reality in cooperation with robotics have been developed as a means of immersing the patient in the task, and motivating them to improve [4,30-32]. One principle of effective neural plastic rehabilitation is the concept of salience which refers to how 
well the therapy connects to tasks associated with daily living. Virtual reality can provide this connection by immersing the patient in a virtual world.

\subsection{Approaches to Haptic Rehabilitation Robotics}

Haptic rehabilitation robots have been developed for both the upper and lower extremities $[25,33]$. These devices are designed to provide the user with a sense of touch throughout the rehabilitation exercise. With regards to salience, haptic feedback can provide a connection with daily tasks by simulating the interaction of objects or surfaces seen in real life. Along the same lines, if a haptic simulation can provide a high level of fidelity, the patient will be more likely to engage in the activity at hand. This engagement is necessary as neural plasticity cannot be achieved without a high level of effort and attention from the patient $[34,35]$.

Haptic rehabilitation devices can be divided into two groups: upper extremity and lower extremity devices. Upper extremity devices target the rehabilitation of the hand and/or arm of a patient. These devices are generally designed to be lightweight and provide relatively small loads to the patient. Lower extremity rehabilitation devices must have a much larger payload to accommodate for the larger muscle groups and in some cases, the weight of the patients. If the payload is larger, the actuation systems must also be larger which in turn causes the devices to be more hazardous to patient safety. For this reason, there are fewer lower extremity devices than upper extremity.

A wide range of both upper and lower extremity devices are assessed below. Although the device presented in this thesis is designed for use with the lower extremities, many concepts found in upper extremity devices are applicable to general haptic rehabilitation such as controls, safety mechanisms, patient training methods, and haptic rendering algorithms. 


\subsubsection{Upper Extremity Haptic Rehabilitation}

\section{HapticKnob}

The HapticKnob is a 2 degree of freedom (DoF) robotic device designed to train grasping of the hand and forearm pronation/supination. The device is designed to retrain specific tasks which are apparent in daily life such as opening a jar, holding a fork, and manipulating a key. The HapticKnob has a workspace of $150 \mathrm{~mm}$ in opening and $30 \mathrm{~mm}$ in closing with a maximum force of $50 \mathrm{~N}$. The device has a rotational range of motion of $\pm 180^{\circ}$ with a maximum torque of $1.5 \mathrm{Nm}$ [1]. The HapticKnob can be seen in Figure 1.1 below.

Lambercy et al. used the HapticKnob as a tool in a 6 week rehabilitation program involving 13 subjects. On average, the subjects demonstrated an increase in grip force and a decrease in muscle spasticity [36].

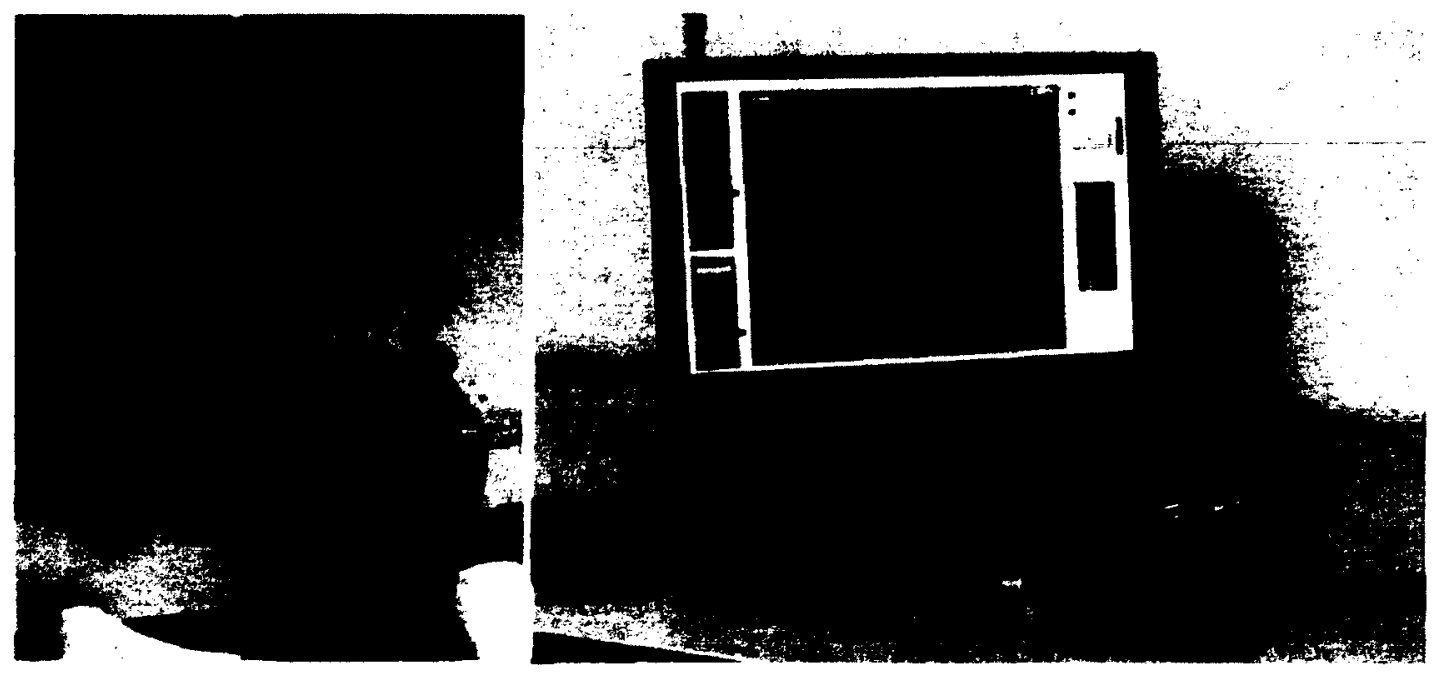

Figure 1.1: The HapticKnob [1]

The task specific nature of this device hinders the range of exercises that it is able to perform such as individual finger fractionation. This device does, however focus on the salience aspect of rehabilitation as it is able to reproduce motions and forces normally seen in everday living (eg. opening a jar). 


\section{Quanser Haptic Device}

Lam et al. developed a 2-DoF haptic rehabilitation robot in collaboration with Quanser Inc., the target exercise for this robot being a reaching forward motion. As can be seen in Figure 1.2 below, there is one rotational DoF moving laterally from the saggital plane and a linear DoF in the plane of the user. The device was designed to be lightweight, collapsable, and adjustable for different users [2].

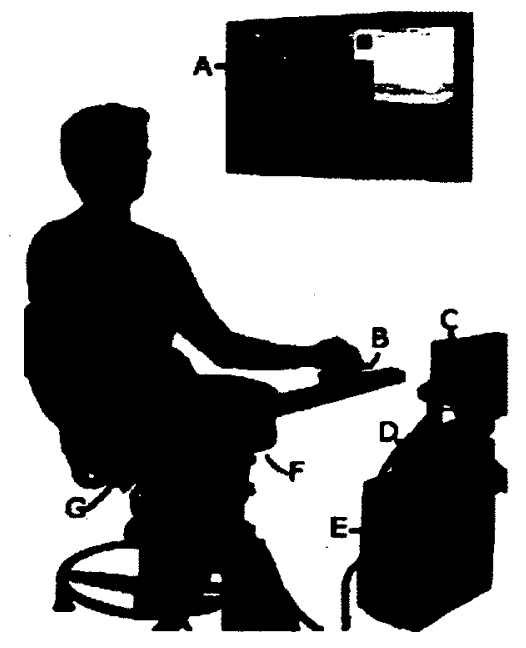

Figure 1.2: The Quanser Haptic Rehabilitation Robot [2]

Lam et al. used the Quanser rehabilitation robot as a rehabilitation tool in a 6 week trial program involving 8 healthy occupational therapists. The device was combined with postural sensors and an elbow stimulation device in order to encourage elbow movement. All therapists felt that the device could be a good tool to use in upper limb rehabilitation as it was capable of delivering a reaching task [2].

Much like the HapticKnob, the Quanser Haptic Device is capable of delivering a task specific motion which is beneficial to the rehabilitation process. A disadvantage of the Quanser Haptic Device is that it is constrained to 2-DoF. The planar nature of the robot simplifies the controls, however it also constrains the out of plane motion which is essential to a highly articulated arm joint. 


\section{PHANTOM OMNI}

The PHANTOM OMNI is a commercialized haptic interface for the hand. It offers 6-DoF positional sensing with 3-DoF $(x, y, z)$ force feedback. The PHANTOM spans a workspace of $160 \mathrm{~mm} \times 120 \mathrm{~mm} \times 70 \mathrm{~mm}$ and is ideal for use in desktop applications with a foot print of $168 \mathrm{~mm} \times 203 \mathrm{~mm}$ [3]. The PHANTOM has been used in many haptic rehabilitation applications because of its low cost, portability, and relatively high precision.

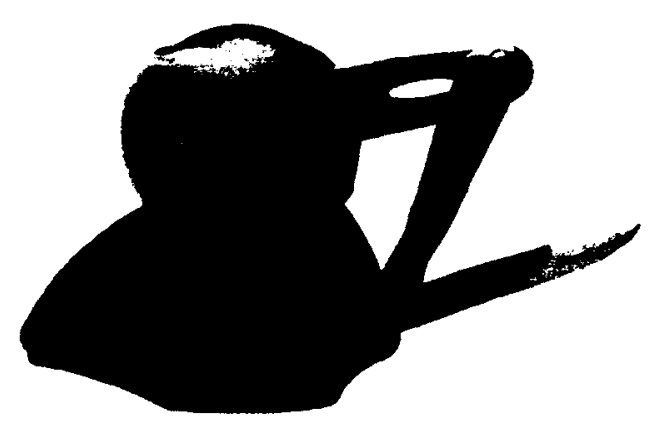

Figure 1.3: The PHANTOM OMNI Haptic Device [3]

Jarillo-Silva et al. developed a haptic training method for the upper arm using the PHANTOM [37]. The patient was asked to maneuver the PHANTOM end-effector through a 2D maze for several trials. First, the patient would maneuver through the maze without any haptic feedback from the device. Next, the patient was asked to manoeuvre the device through the maze with haptic feedback, providing a restoring force if the patient deviates from the path. The patient was then asked to repeat the first trial (without feedback) for comparison. Patient performance was improved by comparing the results of the first and last trial, although it is possible that the improvement could be associated with task repetition rather than training from haptic feedback.

Broeren et al. used a different strategy towards haptic rehabilitation [4]. They combine the PHANTOM with a stereoscopic visualization system, Crystal Eyes CE-2 as seen in Figure 1.4. This visualization system allows the user to view $3 \mathrm{D}$ objects through a special display monitor and stereo viewing glasses. A pilot study was conducted on a single poststroke subject in which he was asked to play a computer game involving 3D bricks. After 12 weeks of treatment, the subject's finger dexterity, grip force, and endurance all increased. 
Although the use of rewarding activities was shown to improve the patient's motivation to practice, it is not concluded whether this treatment is more effective than conventional methods.

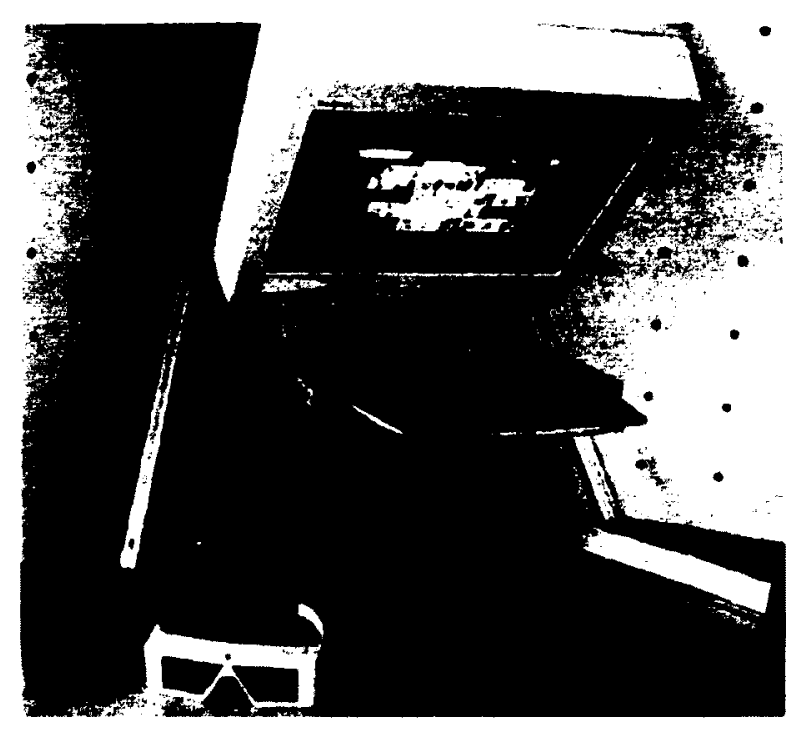

Figure 1.4: The PHANTOM haptic device combined with Crystal Eyes CE-2 setup [4]

The PHANTOM haptic device is ideal for applications involving fine motor control of the hand, however the relatively small workspace prevents the device from retraining large sweeping motions of the arm. The device is also limited in terms of haptic feedback since it is only capable of producing forces, not moments.

\section{Driver's Simulation Environment for Arm Therapy}

The Driver's Simulation Environment for Arm Therapy (SEAT) is a task specific approach to the haptic rehabilitation problem. This device simulates driving an automobile by using the steering wheel as the haptic interface. The SEAT consists of a steering wheel for haptic feedback and graphical interface for visual feedback (Figure 1.5). The steering wheel is split into two halves, each half with its own force sensor. The device is designed to assist stroke survivors with hemiplegia to decrease overuse of the less-affected arm and in turn, increase the use of their impaired arm. This is accomplished by providing constraint induced therapy to stiffen the steering wheel if it is being turned by the strong arm [5]. 


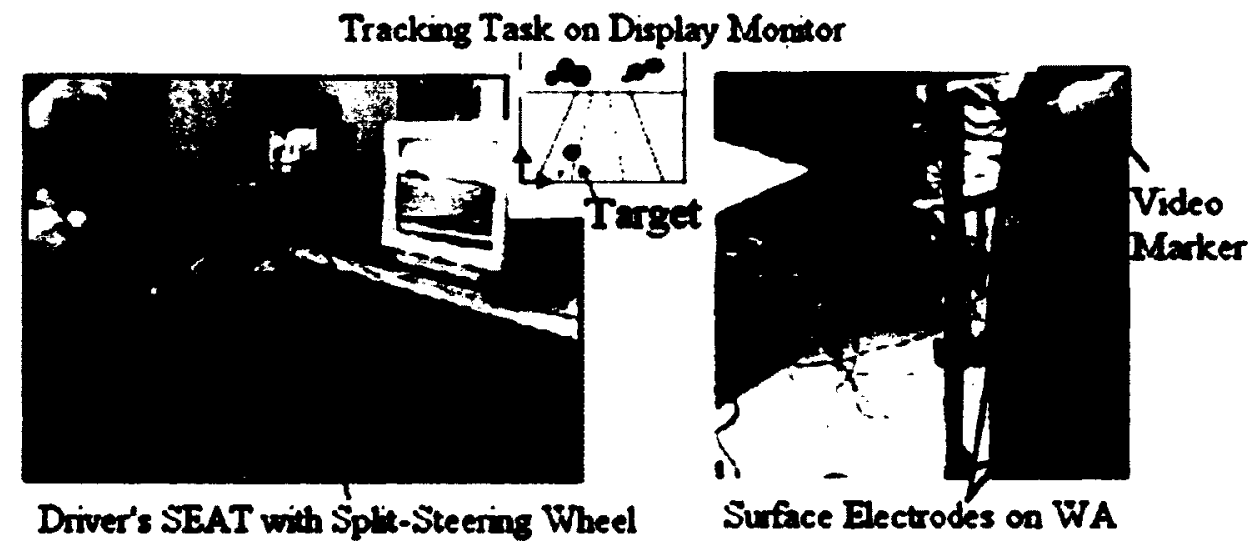

Figure 1.5: The Driver's SEAT haptic device [5]

The Driver's SEAT provides a simple and effective solution for retraining a hemiplegic patient to drive. However, like most of the other task-specific rehabilitation devices, the SEAT lacks in range of exercises that can be performed and thus, can only be used for one purpose.

\section{Rutgers Master II}

The Rutgers Master II (RMII) is a hand based haptic interface that can provide up to $16 \mathrm{~N}$ of force to the thumb, index, middle, and ring fingertips (Figure 1.6). The RMII uses hall effect and IR sensors to record flexion and abduction angles and the translation of the pistons inside the cylinders. The RMII weighs roughly $80 \mathrm{~g}$ which makes it an ideal candidate as a haptic hand device as it does not induce fatigue compared to commercial devices [6]. The RMII glove is designed to train finger strength, range, and fractionation.

Boian et al. and Jack et al. conducted simple experiments with the RMII in order to train four major hand impairments: finger range of motion, finger speed of motion, degree of independence (finger fractionation), and finger strength [30] [32]. Visual feedback was utilized through a graphical user interface which allowed patients to see how well they are progressing. Although the Rutgers Master II device can provide forces to the user's hand, it has only been used as a strength training tool and has not been used as a haptic interface between the user and virtual environment. 


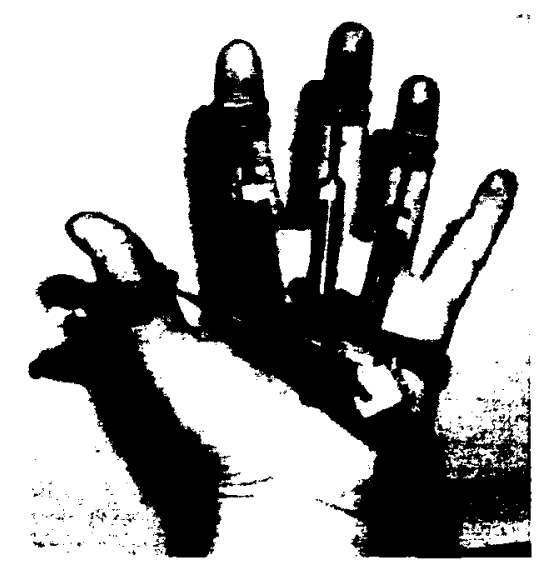

Figure 1.6: The Rutgers Master II prototype [6]

\section{CyberGrasp}

McLaughlin et al. have also conducted research with a haptic device called the CyberGrasp (Figure 1.7). Much like the Rutgers Master II glove, the CyberGrasp is an exoskeletal device which fits over the user's hand and can provide haptic feedback [31]. The CyberGrasp has a 12-bit force resolution and can deliver forces of up to $12 \mathrm{~N}$ per finger [7].

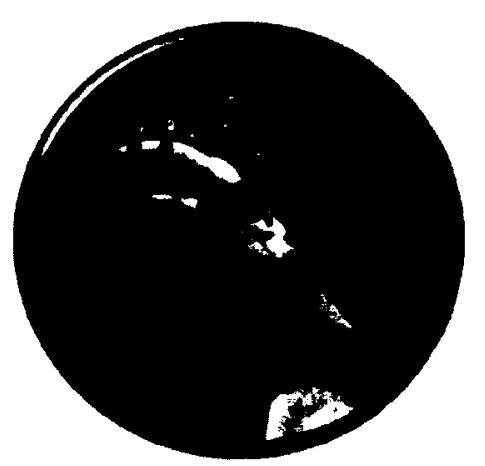

Figure 1.7: The CyberGrasp ${ }^{T M}$ haptic interface [7]

McLaughlin et al. designed exercises for the CyberGrasp in order to provide functional rehabilitation which can be of use in daily life [31]. These exercises include: pouring water into a glass, placing books on shelves, and stacking objects. 


\subsubsection{Lower Extremity Haptic Rehabilitation}

Lower extremity devices can be subdivided into two categories: exoskeletal and end-effector based. End-effector devices have only one point of contact, usually attached to the patient's foot. Exoskeletal devices, however, have multiple points of contact along the human leg which allow for control of each limb segment separately. This section will be focused on the critical evaluation of lower extremity end-effector based devices, however a review on some well known exoskeletal type devices that are capable of haptic feedback are presented at the end of this section as well.

\section{HapticWalker}

There are very few end-effector based lower extremity rehabilitation robots with the capability of providing ground reaction forces due to the large payload required. As far as we know, the research group that developed the HapticWalker is the only group who has taken a step towards reproducing ground reaction forces through a haptic device.

The HapticWalker is a device which provides a haptic interface with a patient by coupling each foot to a foot plate. These foot plates are attached to serial robot linkages which provide 3-DoF in the sagittal plane as seen in Figure 1.8. Force/Torque sensors mounted on each foot plate allow the HapticWalker to operate in a force controlled mode. The HapticWalker's workspace dimensions are $0.9 \mathrm{~m}$ step length, $0.4 \mathrm{~m}$ step height, and $-30^{\circ}$ to $+90^{\circ}$ foot flexion and extension. The device has a maximum user weight of $120 \mathrm{~kg}$ and a maximum walking speed of $5 \mathrm{~km} / \mathrm{h}$. To ensure patient safety, an active suspension system is used to prevent falling and for body weight support during operation. Along with the suspension system, limit switches, emergency stops, software stops, and mechanical release mechanisms similar to those in ski bindings have been implemented as a means of preventing harm to the user [38].

Two position control modes have been developed which assist the user during a normal gait cycle. The treadmill mode follows a predefined trajectory which relates to normal gait patterns derived from motion tracking data. The Center of Mass (CoM) mode attempts to 


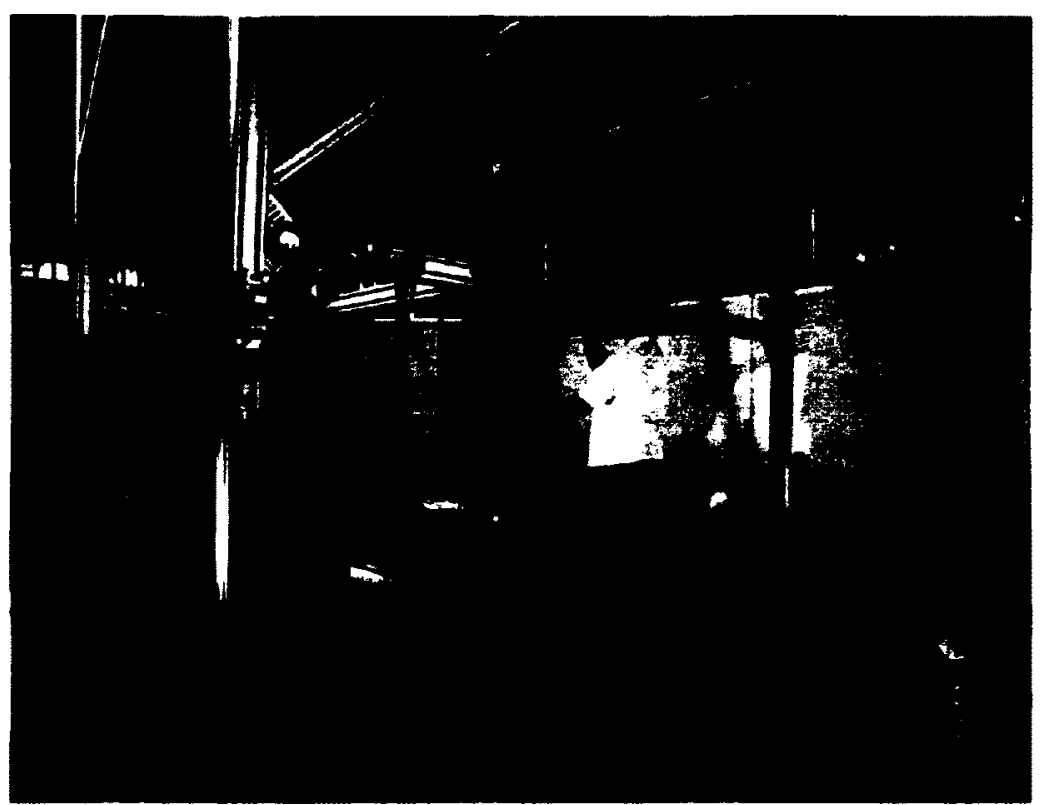

Figure 1.8: The HapticWalker [8]

hold the user's center of mass at a constant vertical position in order to actively support the body during the gait cycle [9]. The gait trajectories for the treadmill and CoM modes can be seen in Figure 1.9. These control modes drive the patient's feet through a desired gait trajectory. The forces that are generated cannot be indicative of ground reaction forces that are normally seen through walking since the patient is relying on the robot to provide motion.
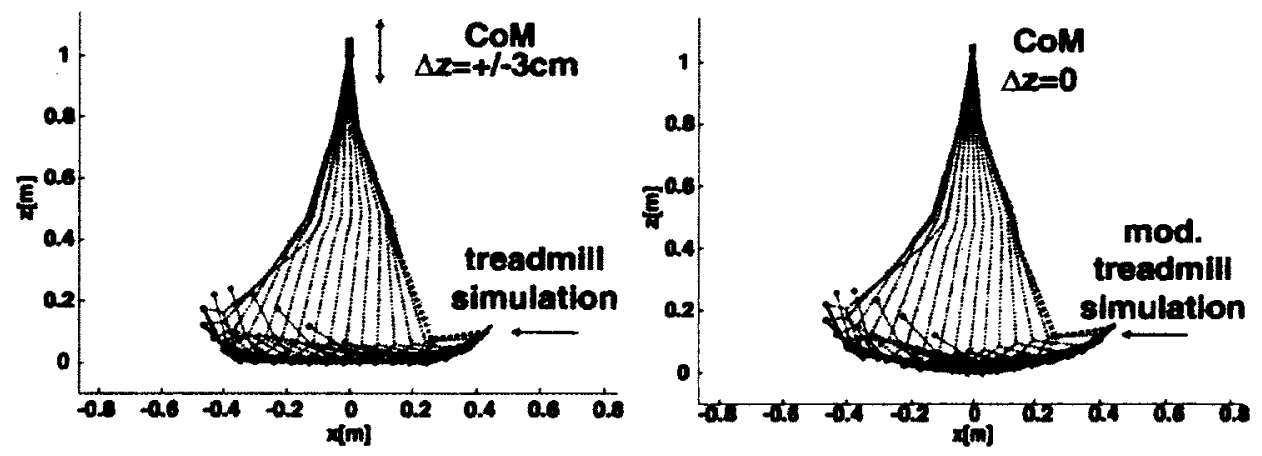

Figure 1.9: Motion tracking data for the Treadmill mode foot trajectory and CoM mode foot trajectory [9]

A haptic framework has also been implemented on this device which allows the user to 
interact with a virtual treadmill. The user's stance foot is dragged backwards as the swing foot is allowed to move freely. Ground reaction forces produced by several gait cycles are shown in Figure 1.10. The magnitude of the force in the vertical direction proves that the device is capable of generating ground reaction forces proportionate to the human operator. However, the vertical ground reaction profile is skewed from one that is seen through normal walking. Also, results show that during the swing phase, forces acting on the foot in the horizontal direction range from $50-100 \mathrm{~N}$ [10]. These forces are significant when relating it to the muscle impairment of hemiparetic patients.

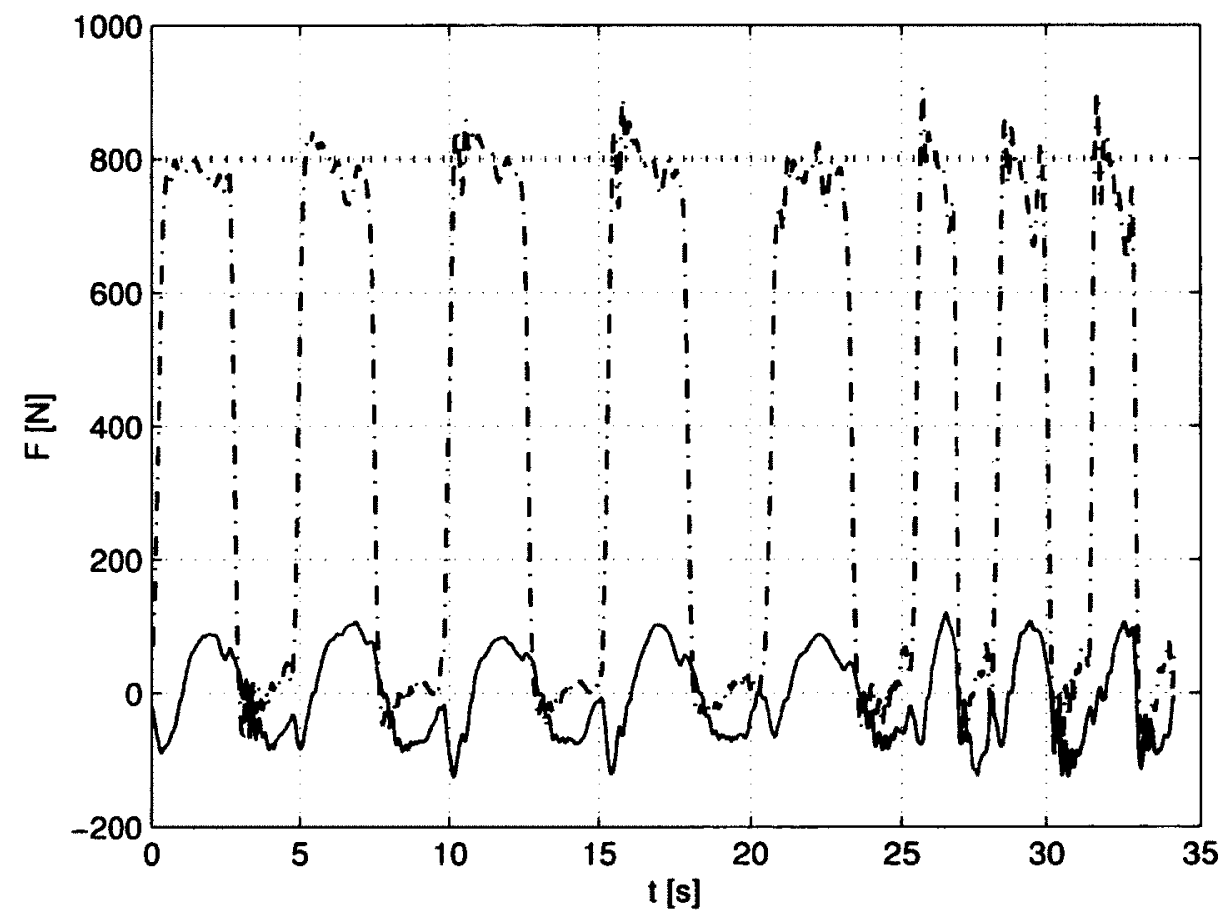

Figure 1.10: Vertical and horizontal forces acting on a users foot. $F_{\text {vert }}$ (Dashed-dotted black) and $F_{\text {horiz }}$ (solid blue). User body weight is indicated by the red dashed line [10].

\section{Rutgers Ankle}

The Rutgers Ankle (RA) is a haptic interface that attaches to the user's foot with a foot plate while seated. The platform consists of 6 , dual acting pneumatic cylinders which are placed in a Stewart platform configuration (Figure 1.11). The RA can provide forces of up 
to $371 \mathrm{~N}, 316 \mathrm{~N}$, and $752 \mathrm{~N}$ with maximum displacements of $12 \mathrm{~cm}, 9 \mathrm{~cm}$, and $12 \mathrm{~cm}$ in the $X$, $Y$, and $Z$ directions respectively. The parallel manipulator design makes the RA an ideal device for providing large forces, however the workspace of such a design is limited compared to the range of motion of a human leg. There is a 6-DoF Force/Torque sensor located under the foot plate which measures the forces being applied from the user and provides motions based on those forces. A virtual world was created with WorldToolKit (WTK) which provides a visual representation of the rehabilitation exercises such as accelerating a racecar or controlling the orientation of a plane $[11]$.

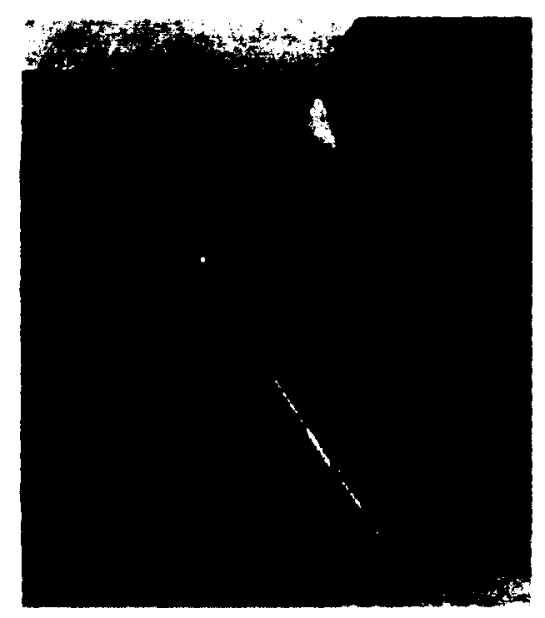

Figure 1.11: The Rutgers Ankle haptic platform [11]

The RA is intended to be used at home as a telerehabilitation device by sending relevant patient data to Rutgers University for observation. In order to increase patient immersion, several effects have been implemented by the RA such as varying the stiffness of the device and adding vibrations [12].

Clinical trials have been conducted using the RA interface shown in Figure 1.12. Patients were asked to accomplish certain tasks while being subjected to haptic effects such as turbulence and jolts. Although the haptic effects have not been proven to aide in the rehabilitation process, they did not interfere with the ability to perform the desired exercises [12]. 


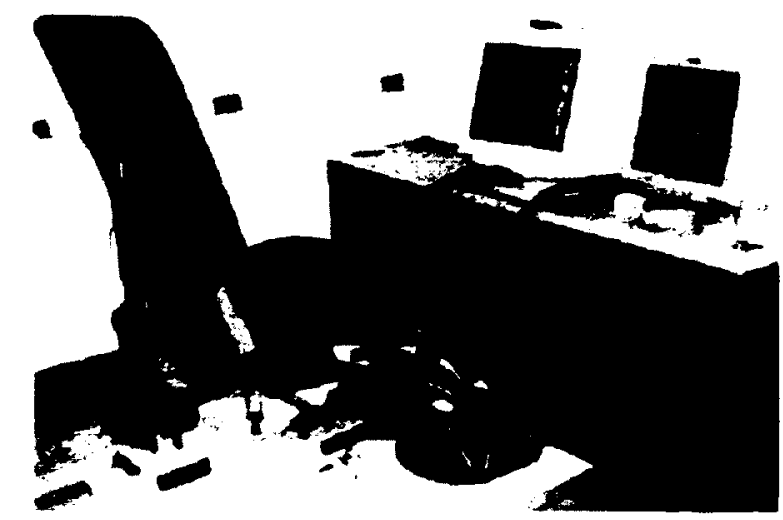

Figure 1.12: Two Rutgers Ankles in a rehabilitation setup [12]

\section{Rutgers Mega Ankle}

The Rutgers Mega Ankle (RMA) is an extension to the Rutgers Ankle platform. Two modified Rutgers Ankle platforms are placed shoulder width apart allowing the user to rigidly attach their feet to each foot plate as seen in Figure 1.13. The user's body weight is being supported by the platforms, however there is a harness which acts as body weight support in case the user loses balance. The range of motion of the RMA allows for $30 \mathrm{~cm}$ of displacement in the horizontal direction and $12 \mathrm{~cm}$ of vertical displacement with a minimum vertical force across the entire workspace of $3150 \mathrm{~N}$. The RMA can move $\pm 25^{\circ}$ in pitch and roll and $\pm 40^{\circ}$ in yaw [13]. Although the RMA is capable of operating with a patient of up to $300 \mathrm{~kg}$, it suffers from the same reduced workspace as the RA. The maximum horizontal displacement of the RMA is $30 \mathrm{~cm}$ whereas a normal male subject has an average stride length of $1.5 \mathrm{~m}[21]$.

The RMA employs visual feedback to the user through a large monoscopic display. During the virtual walking simulation, the user is asked to walk across a virtual crosswalk (Figure 1.14) as the RMA transitions into separate states depending on the position of the user's foot. When a foot is elevated off the virtual ground, the control switches to zero impedance mode and attempts to produce transparent motion. Once that foot regains contact with the virtual ground, the control mode switches to position control and slides the foot backward, simulating a treadmill [13]. Much like the position control mode on the 


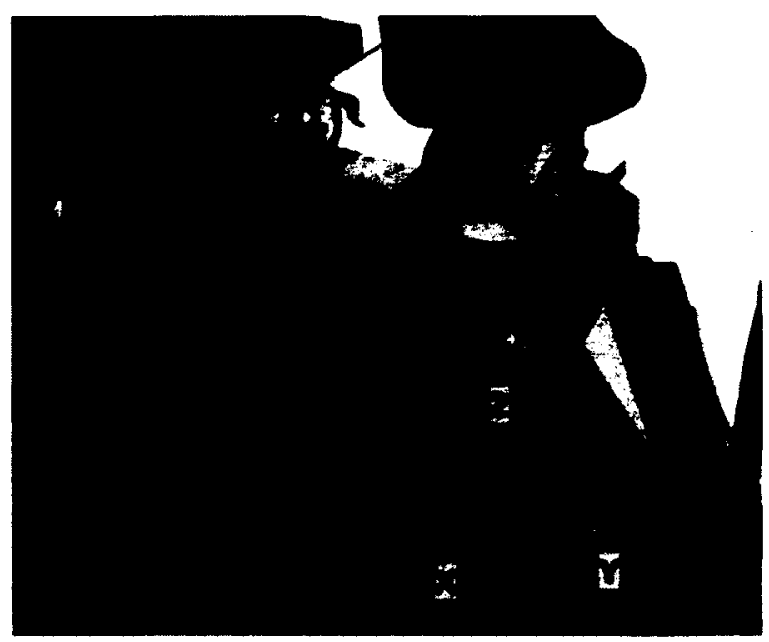

Figure 1.13: The Rutgers Mega Ankle foot plates [13]

HapticWalker, the ground reaction forces generated cannot be used quantitatively to assess the gait of a patient.

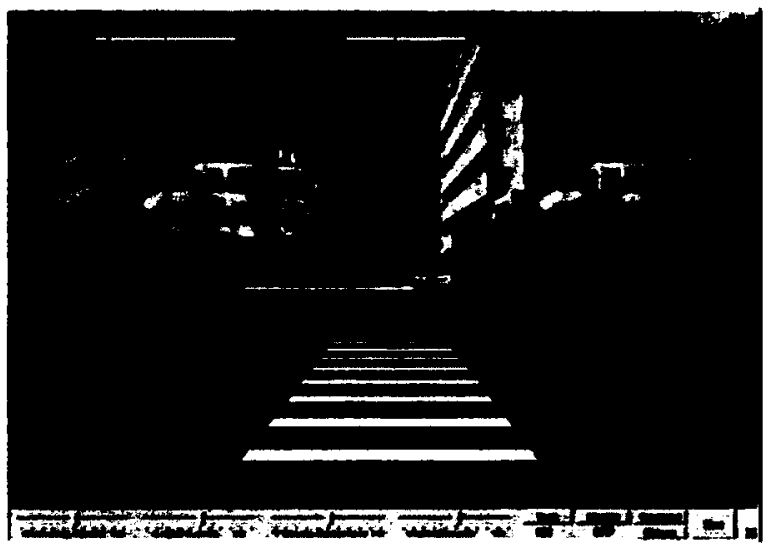

Figure 1.14: The Rutgers Mega Ankle Virtual Crosswalk [14]

\section{Cable-Driven Locomotion Interface}

The Cable-Driven Locomotion Interface (CDLI) is a haptic interface for the lower extremities which provide independent, 6-DoF motion for each foot. The CDLI consists of two foot platforms which are driven by eight cables each in order to control 6-DoF. As can be seen in Figure 1.15, the user's feet will be rigidly attached to each of the platforms during operation. The linear workspace of the CDLI in the $\mathrm{X}, \mathrm{Y}$, and $\mathrm{Z}$ directions are $2 \mathrm{~m}, 0.6 \mathrm{~m}$, and $1 \mathrm{~m}$ 
respectively and the angular workspace in the $\phi, \theta$, and $\psi$ directions are $\pm 45^{\circ}, \pm 45^{\circ}$, and $\pm 20^{\circ}$ respectively [39]. Although in theory cable driven devices benefit from low inertia, high speed, high acceleration, and a large workspace, the CDLI is still in development and has yet to publish specifications regarding these areas.

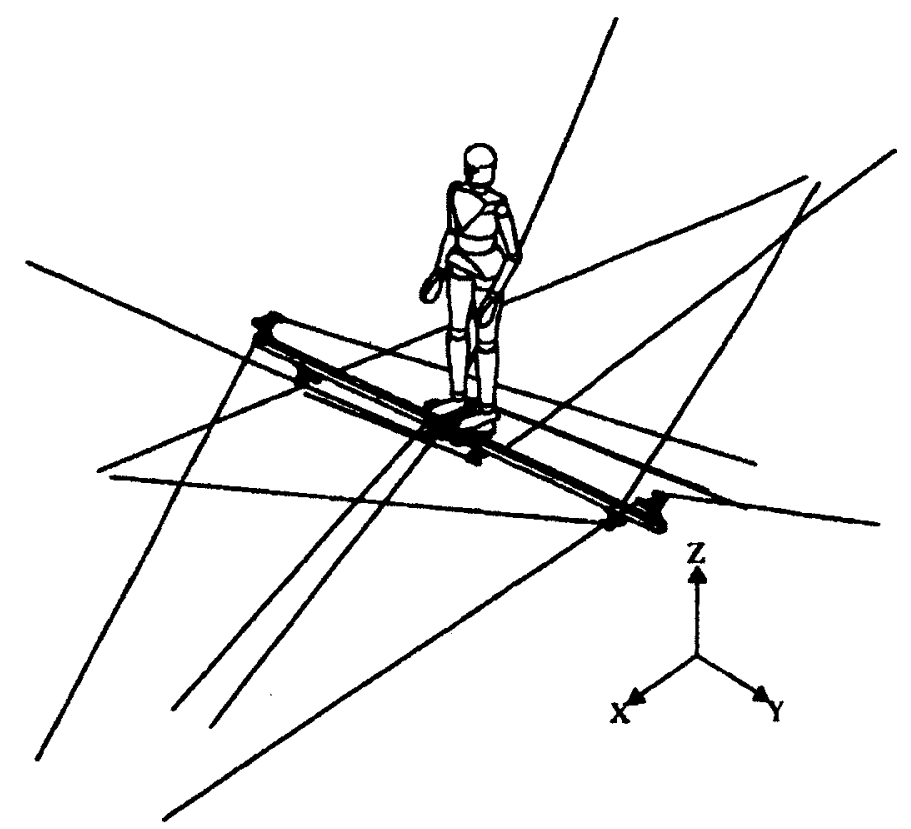

Figure 1.15: A CAD model of the CDLI [15]

The nature of cable driven platforms require the cables to be actuated at all times in order to prevent slack. During operation, it is possible for the tension in a cable to drop, forcing the other cables to a high tension state and causing oscillations. Control strategies have been designed and simulated for the CDLI which attempt to remedy this behaviour [39].

An admittance control architecture for haptic rendering was developed for the CDLI in combination with the Newton Game Dynamics ${ }^{T M}$ engine as well as H3D [40] and ODE [19] software for collision detection and force computation [15]. This control scheme, combined with the CDLI simulation were used to generate the forces and torques necessary to deliver the feeling of walking on flat ground. Since the CDLI is still in the development phase, no user data is available at this time. 


\section{6-DoF Gait Rehabilitation Robot}

This 6-DoF gait rehabilitation robot is the only device reviewed which attempts to rehabilitate both the upper and lower body. The main objective of this device is to retrain gait symmetry and posture stability during wakling. The patient's feet are rigidly attached to foot pads with 3-DoF: vertical displacement, foot to heel displacement, and ankle rotation (Figure 1.16). This device supports a maximum step length of $0.8 \mathrm{~m}$ and a maximum step height of $0.18 \mathrm{~m}$ with a maximum velocity of $0.3 \mathrm{~m} / \mathrm{s}$. Also, visual feedback is provided through an LCD screen at the front of the device using the Labview Picture 3D toolkit to create a virtual environment [16]. In an attempt to simplify the walking process, both the motion of the feet and arms are kept in phase through a timing belt. It should be noted that this coupling could cause deviations from a normal gait cycle. Although force/torque sensors are embedded in the foot pads, this device operates primarily in position control mode. 


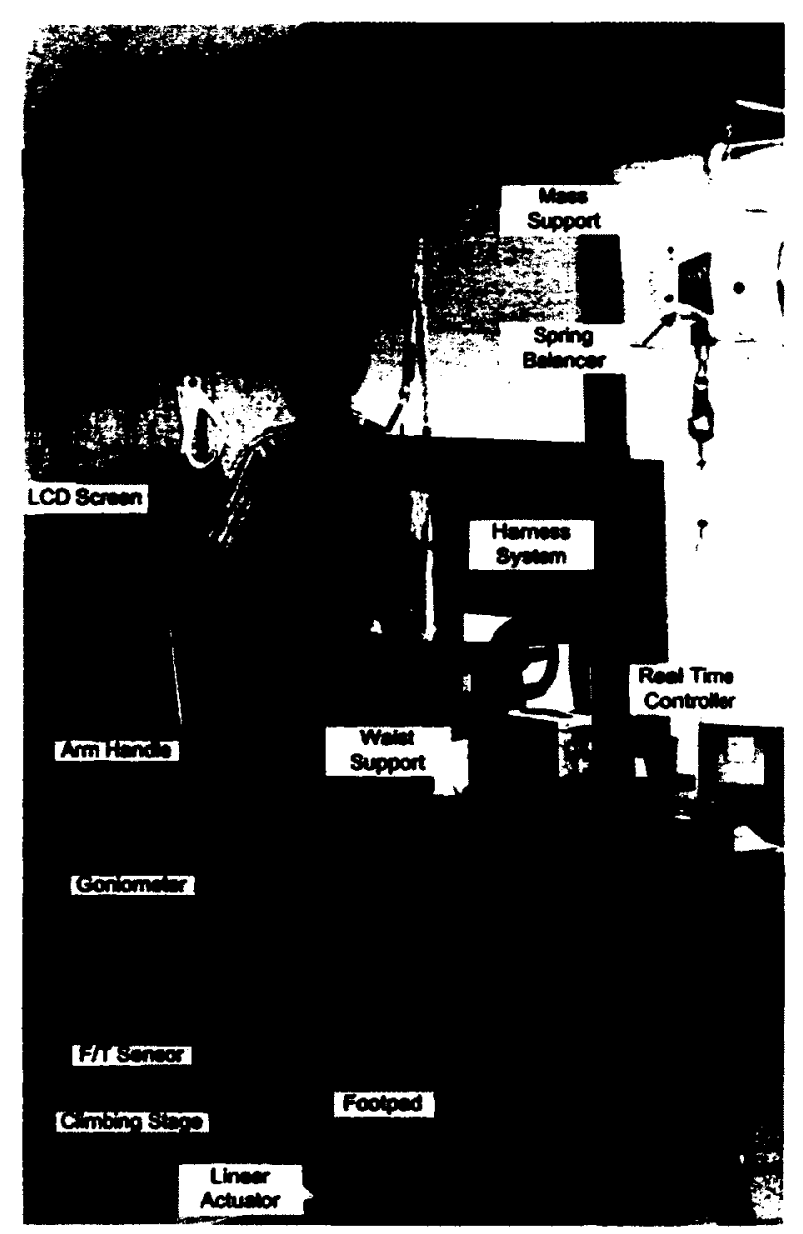

Figure 1.16: CAD model of the 6-DoF rehabilitation robot [16]

\section{Gait Master}

Much like the design of the Rutgers Mega Ankle, the Gait Master (GM) employs the use of two parallel manipulators in order to simulate uneven surfaces and stairs during walking (Figure 1.17). While climbing stairs, the GM uses a washout algorithm such that the user's centre of mass stays constant, much like an exercise stairmaster. The GM has a total horizontal workspace of $32 \mathrm{~cm}$ front to back, and $28 \mathrm{~cm}$ side to side and a vertical workspace of $20 \mathrm{~cm}$ which again, does not allow for a full stride length. The user can, however rotate their feet in the horizontal plane by up to $90^{\circ}[17]$.

The primary focus of the Gait Master is directed towards stair climbing ground reaction 


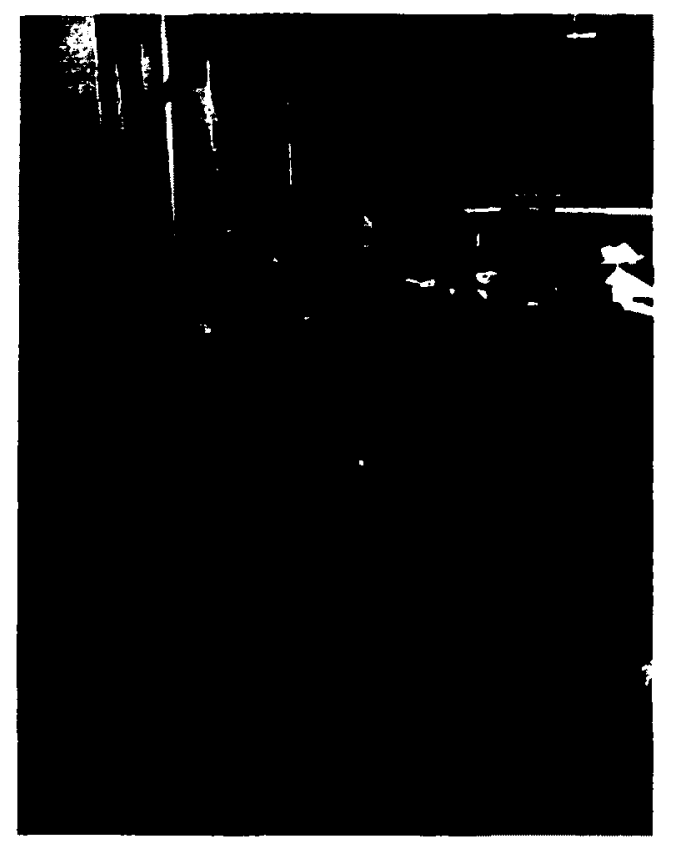

Figure 1.17: The Gait Master prototype [17]

forces. Force profile data from a single patient climbing up a set of stairs was matched with the data from the Gait Master and can be seen in Figure 1.18(a) below. Although slightly skewed, the GM pressure profile matches quite well with the real stair data. However, the GM was not able to match the force data from climbing down a set of stairs (Figure 1.18(b)) which the author attributes to partial weight support from the safety bar. 


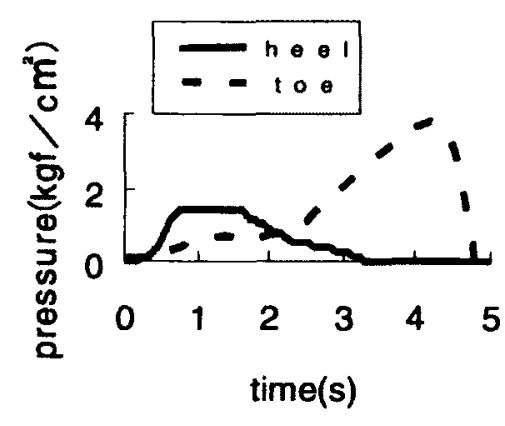

Gait Master

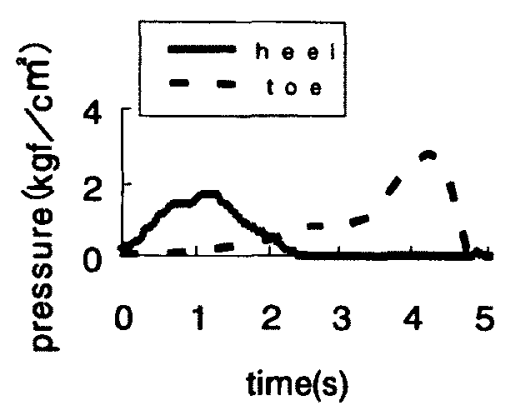

Real stair

(a)

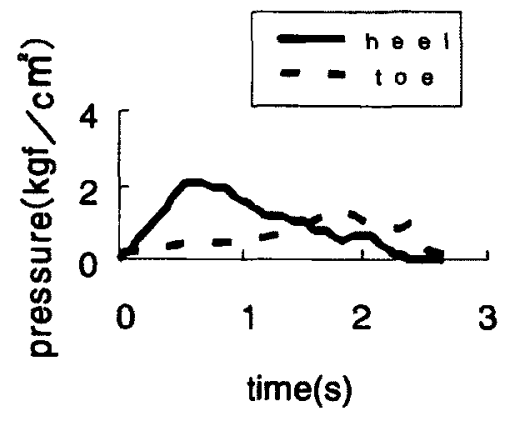

Gait Master

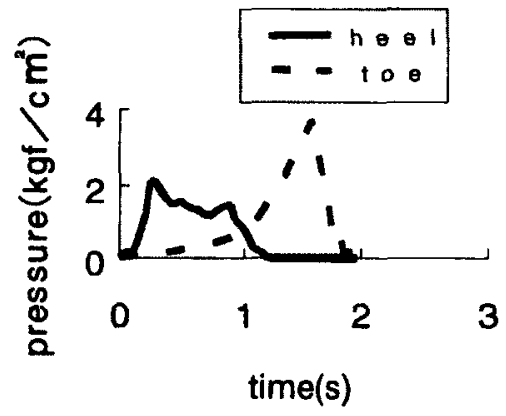

Real sitar

(b)

Figure 1.18: Pressure profiles of GM and real stair data (a) climbing up stairs (b) climbing down stairs [17].

\section{Exoskeleton Type Rehabilitation Robots}

Other lower extremity rehabilitation robots fall into the category of exoskeleton type. These robots generally make use of body weight supported treadmill training (BWSTT) which is now considered a beneficial practice for gait impairments caused by CNS injuries $[41,42]$. There are three well known exoskeleton type devices that are of particular note: Lokomat, LOPES, and ALEX (Figure 1.19).

Lokomat is a driven gait orthosis that automates locomotion therapy on a treadmill. 
Lokomat was initially designed to move patient limbs through desired kinematic trajectories in order to eliminate asymmetrical gait patterns [43]. Findings from the initial studies however revealed that although accurate kinematic patterns were maintained, joint torque patterns were abnormally asymmetric which is a characteristic of hemiparetic stroke subjects. Lokomat was later redesigned to include force sensors at the hip and knee joints which measure the interaction forces between the patient and device. This enabled the employment of a simple haptic rendering algorithm with variable spring and damper constants. Tests concluded that although the device is capable of operating as a haptic device, the bandwidth and force output are very low compared to end-effector type devices [44].

LOPES, or lower extremity powered exoskeleton, is a device which is attached to the patient's lower limbs while walking on a treadmill. The device was designed to act in two main modes: patient in charge and robot in charge. In patient in charge mode, the robot is set to act as transparent as possible while the patient walks. Robot in charge mode attempts to drive the patient's limbs through a desired trajectory. A third mode, named therapist in charge mode, is a combination of the two in which corrective or selective torques can be applied to a patient's limbs as they attempt to follow a predefined gait pattern [45].

The active leg exoskeleton, or ALEX is a powered orthosis for the lower limbs. Much like Lokomat and LOPES, ALEX is designed to function during treadmill training of a patient. ALEX is fitted with force/torque sensors in order to implement control algorithms with force feedback. A force-field control strategy has been developed for ALEX which gives a desired foot trajectory to the patient. If the patient deviates from the nominal trajectory, an assitive force guides the patient back [46]. 


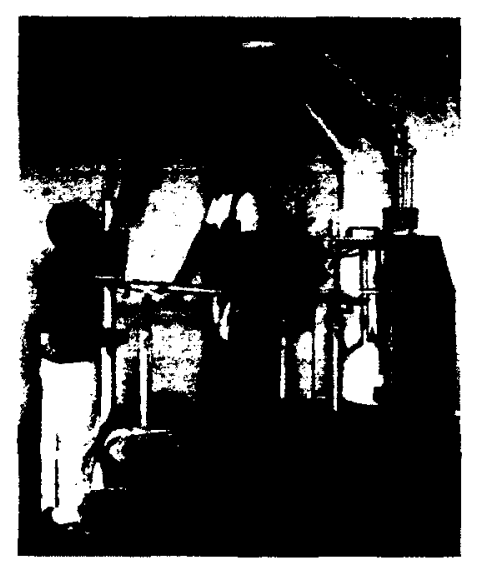

(a) LOKOMAT

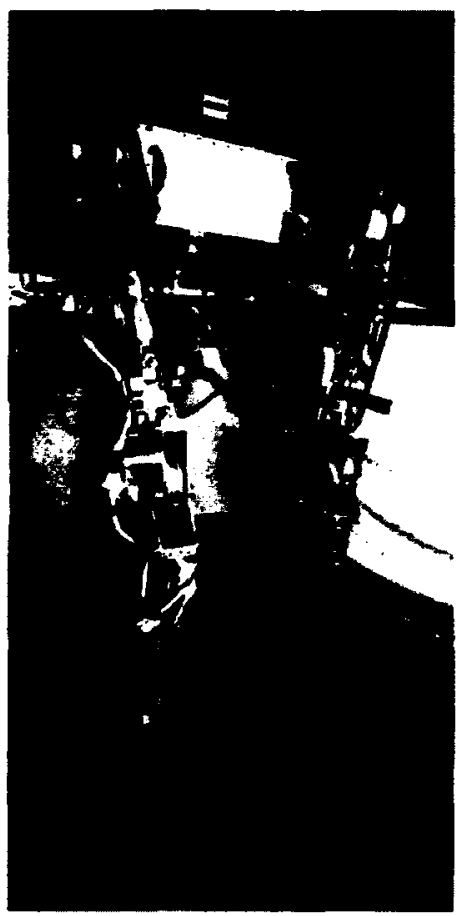

(b) LOPES

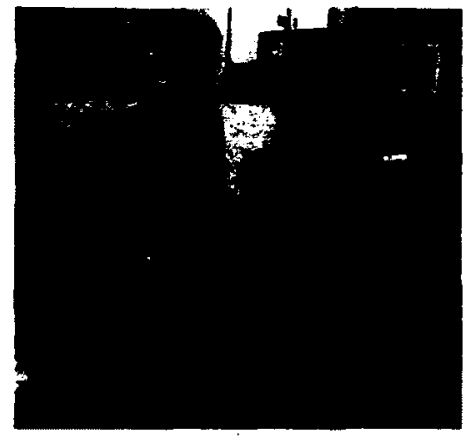

(c) ALEX

Figure 1.19: Exoskeletal type rehabilitation robots

\subsection{Platform Introduction}

As outlined above, there are very few lower extremity devices that provide a large workspace to accomodate for a variety of patient sizes, while still maintaining the ability to generate relatively large contact forces and torques to the patients foot.

\section{Previous Approaches}

Parallel manipulators such as the Rutgers Mega Ankle and Gait Master are designed to deliver high loads, however they suffer from a limited range of motion. Cable driven platforms such as the CDLI are capable of delivering high fidelity haptics due to their low inertia, however the complexity of the systems and inherent instabilities prevent them from developing past the conceptual phase. Exoskeleton type devices are another approach towards lower extremity haptic rehabilitation and are designed for applications which require individual joint control of the patient. These devices 
can be difficult to design for patients of varying sizes and could cause injury due to misalignment. Also, the haptic display properties of exoskeleton type devices are limited since they are unable to generate forces and torques at the foot-ground interface. The most versatile lower extremity haptic device in terms of gait rehabilitation is found to be the HapticWalker. This end-effector type device is capable of producing ground reaction forces over a large workspace. The disadvantage, however is that it is limited by how transparent the device can be during the swing phase of the gait. A highly transparent haptic device requires less force to generate a motion.

\section{Lack of Versatility}

A major caveat with almost all of the devices listed above is the limited range of motion and versatility associated with each device. Most of the devices were designed with a specific task to be executed. Conventional rehabilitation has proven that the patient must be actively engaged in the rehabilitation task with a high level of attention and effort. A device which provides only a single task can quickly become tiresome and repetitive.

\section{Virtual Gait Rehabilitation Robot}

The Virtual Gait Rehabilitation Robot (ViGRR) is a platform that is motivated by the need for a lower extremity haptic rehabilitation robot which can provide a wide range of therapy to patients who have been affected by a central nervous system disorder. The novelty of the ViGRR platform is that it provides therapy to severely impaired patients within days after being diagnosed with a CNS disorder. This time has been proven as the most effective time for neural plastic rehabilitation [49]. The ViGRR provides a safe environment to rehabilitate the lower extremities without the danger of falling or injury by allowing the patient to remain in the seated position. The original concept for the ViGRR platform was designed by two previous Master's students at the Advanced Biomechanics and Locomotion (ABL) lab at Carleton University $[47,48]$. Their initial design involved coupling the feet of the patient to the end-effectors of two, planar serial robots as seen in Figure 1.20. The use of serial robots allows for a 
large workspace, while also maintaining high force capacity at the end-effectors.

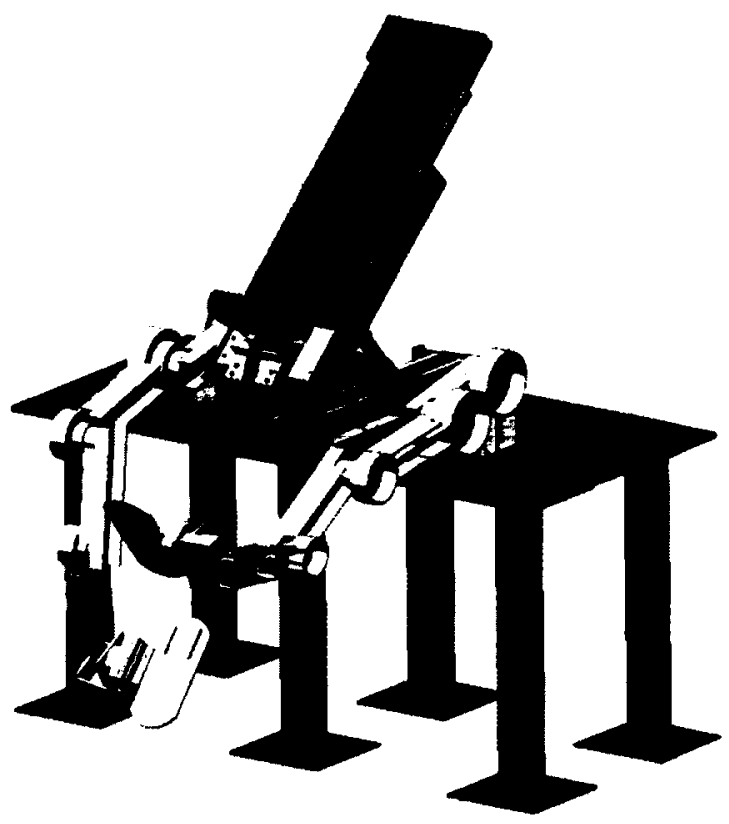

Figure 1.20: Concept CAD model of the ViGRR platform

In order to test some of the major mechanical and software concepts for implementation on the full scale, a ViGRR prototype was built which consists of a single serial robot for the right leg. Along with the mechanical design and manufacture of the robot, a preliminary safe interaction end-effector was designed but not tested due to time constraints. On the software side, a high level control architecture was developed in Simulink which handles all aspects of ViGRR control and safety. Several controllers were designed for the ViGRR prototype in both joint space and task space along with an admittance scheme for force control. Validation experiments were performed with the admittance control using a hand as the patient coupling to show that a trajectory could be followed. A full description of the ViGRR prototype is provided in Chapter 2. 


\subsection{Objectives}

As it stands, ViGRR is a serial robot that is capable of interacting with a user's hand through a force controller. There are several hardware and software developments necessary in order to develop ViGRR into a lower limb haptic rehabilitation device.

\section{Hardware Improvements}

At all times, safety of the user while operating ViGRR is of the utmost concern. Therefore, in order to ensure a safe interaction, an end-effector which will detach to prevent harm to the user must be designed and manufactured. Also, in order for the user to couple with the end-effector, an adjustable seat must be designed which will allow for safe and comfortable operation with ViGRR. Several low level safety features have been implemented to prevent the robot from damaging itself, however these features are solely reliant on the smooth operation of the running software.

\section{Haptic Development}

In order for ViGRR to be an effective tool for lower extremity rehabilitation, a scaled version of the ground reaction forces seen during a normal gait cycle must be reproduced. A simple admittance control like the one implemented on the ViGRR prototype is capable of providing a force display to the user, however it is not able to detect collisions or display the corresponding forces. A haptic rendering algorithm would have the ability to sense different objects in a virtual world and provide forces to the user depending on the position and orientation of those objects. This technique can not only generate ground reaction forces during gait training, but also provide a framework for developing interactive exercises for use with other training methods.

The general objectives of this research were chosen to address some of these outstanding challenges which can be summarized as follows.

1. Improve the hardware and software design of the ViGRR platform to enhance it's technical capabilities and safety features that are relevant to human experimentation. 
2. Develop a haptic framework which will be used with ViGRR to replicate a scaled version of ground reaction forces in a normal gait pattern. This framework must be variable to allow for different types of surfaces, friction, and walking speeds.

3. Present the ViGRR prototype as a platform which can be used as a lower extremity rehabilitation device by developing preliminary biomedical experiments and exercises.

\subsection{Contributions}

The following research contributions are presented in this thesis:

\section{ViGRR hardware development:}

(a) A magnetically detachable end-effector for safe human-robot interaction is designed, manufactured, and tested.

\section{Development of a haptic controller for foot-ground interaction:}

(a) A 6-DoF haptic rendering algorithm is developed and implemented in order to generate forces and torques at the robot end-effector.

(b) Experiments with several virtual tool profiles in conjunction with the algorithm verify that the controller satisfies the haptic requirements.

\section{Experimental Validation:}

(a) Walking experiments involving a healthy subject confirms that scaled ground reaction forces can be observed depending on the virtual tool geometry.

(b) Experiments with virtual reality exercises are conducted in order to highlight the capabilities of ViGRR with respect to lower extremity rehabilitation. 


\subsection{Outline of Thesis}

The rest of the thesis is structured as follows:

\section{Chapter 2: Virtual Gait Rehabilitation Robot (ViGRR)}

This chapter describes the overall design of the ViGRR platform along with an outline of the necessary improvements required for ViGRR to be used as a rehabilitation research platform. In particular, the design of a safe, magnetically coupled end-effector will be presented along with its performance data.

\section{Chapter 3: Haptic Delivery}

This chapter details the process implemented to provide ground reaction forces to the user. A list of requirements for the ViGRR are defined with respect to haptic delivery. A 6-DoF haptic rendering algorithm is developed and implemented for a real-time QNX operating system. Validation experiments regarding the haptic rendering algorithm are presented as well.

\section{Chapter 4: Experimentation}

The focus of this chapter is the development and demonstration of preliminary biomedical experiments relevant to lower extremity rehabilitation. These experiments determine the ability of ViGRR to reproduce the swing and stance phases of a normal gait cycle involving a single subject. Several haptic rehabilitation exercises are presented as well.

\section{Chapter 5: Conclusions and Future Work}

A summary of the completed work as well as a list of future tasks is outlined in this chapter. 


\section{Chapter 2}

\section{Virtual Gait Rehabilitation Robot (ViGRR)}

This chapter focuses on the overall development of the ViGRR platform from its inception to its initial prototype. Several enhancements were required on the initial prototype in order to realize ViGRR as a lower limb haptic rehabilitation platform. This chapter identifies the main issues and attempts to address them through hardware and software modifications.

\section{$2.1 \quad$ ViGRR Introduction}

\subsubsection{Concept}

As stated in Chapter 1, the Virtual Gait Rehabilitation Robot (ViGRR) concept is a platform designed for lower limb rehabilitation of a patient who has been affected by a central nervous system disorder. Due to the severity of the affliction, the patients will have little to no use of their legs, making standing a challenge and in some cases, impossible. ViGRR is a platform that was designed to rehabilitate patients such as these at the earliest stage of rehabilitation, which is proven to be the most critical time for effective therapy [49].

One of the main challenges with rehabilitation is the level of engagement that a patient is willing to contribute. ViGRR is able to achieve this engagement through real-time integration of tactile and visual feedback. A high level overview of the ViGRR platform, as seen in Figure 2.1, can be broken down into 3 sections: robotic system, real-time software, and human-robot interaction. The mechanical aspect of ViGRR consists of the rehabilitation robot, sensors, and the patient-robot interface. The real-time computation section 
encompasses all aspects that are related to robot control including robot kinematics, dynamics, and low level joint control. All aspects of the ViGRR platform that are related to enhancing the immersion of the patient are grouped in the human-robot interaction section. These modules are combined to perform a wide variety of tasks which will not only aid the therapist, but will also keep the patient motivated.

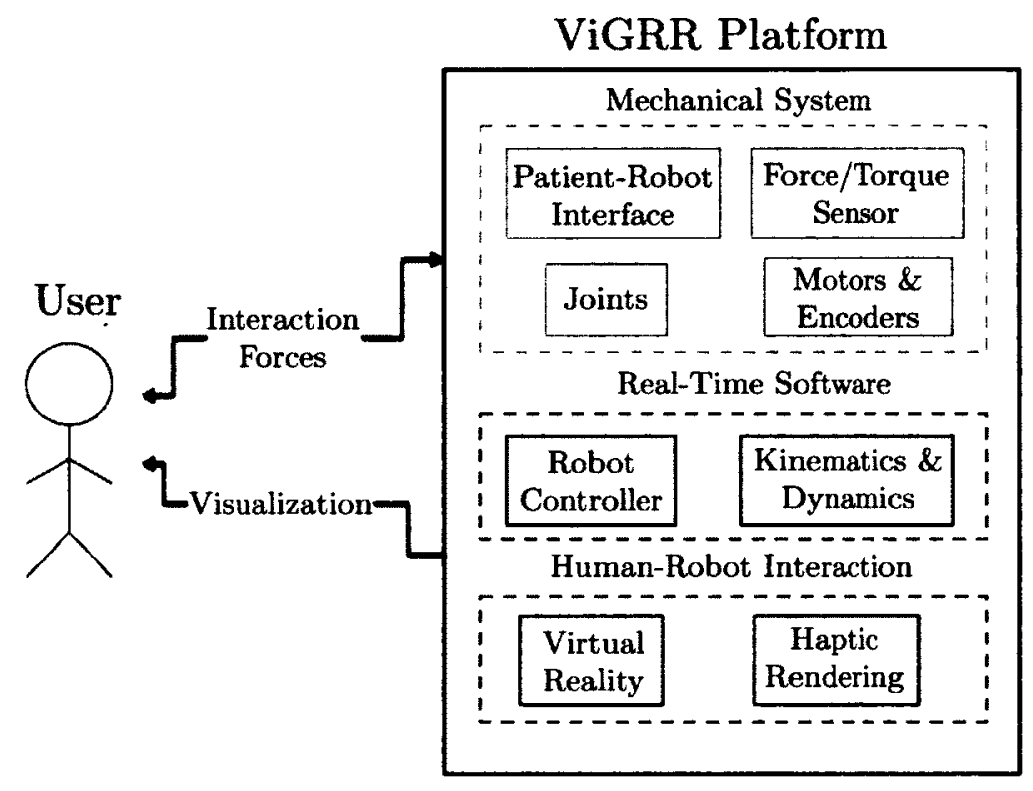

Figure 2.1: Block Diagram of the ViGRR platform.

The initial concept proposes to have the patient in a seated or lying position, with a dedicated 4-link planar robot attached to each foot as seen in Figure 2.2. For severely impaired patients, the gravitational effect of their body weight is reduced by orienting the seat horizontally. However, as the patient gains strength, a portion of their body weight can be introduced by increasing the seat angle. The patient seat also includes a sliding mechanism which allows the seat to translate along the direction of the patient's torso, enhancing the fidelity of gait training. Future iterations of the concept will be extended to 6-DoF for full compatibility with the human leg kinematics however the initial planar design adds a level of safety by mechanically limiting the workspace of the robot.

Although other lower extremity rehabilitation devices have been developed before, the 


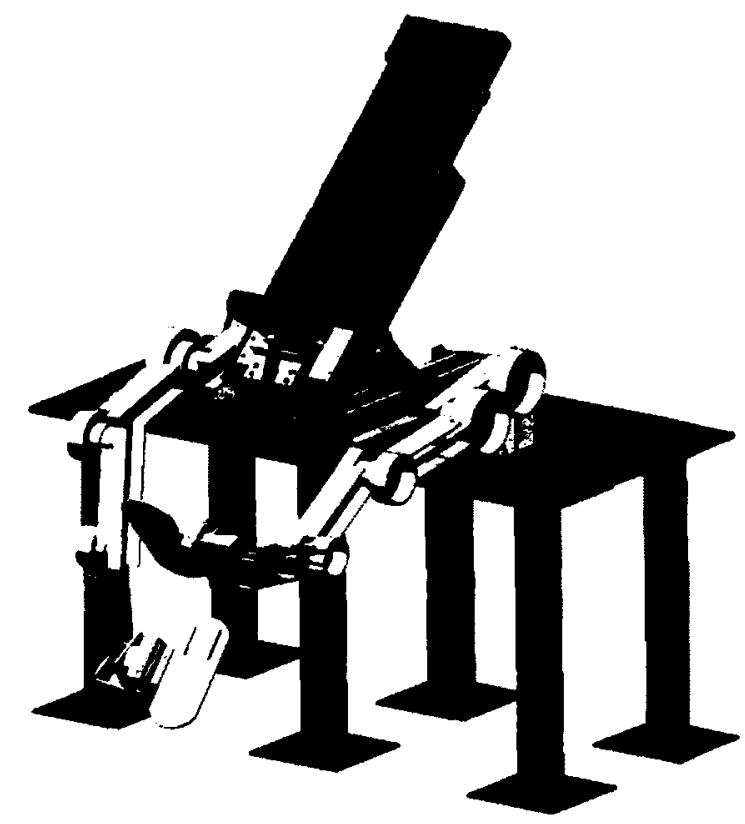

Figure 2.2: Concept CAD model of the ViGRR platform.

novelty of the ViGRR design stems from several key features which contribute to the versatility of the platform. ViGRR combines all of these features to adapt to the rehabilitation task which best suits the needs of the patient.

\section{Workspace}

The workspace and loading requirements of ViGRR were chosen in order to accomodate the heights and masses of a large male (95th percentile) and small female (5th percentile). Not only does this design enable the end-effector of the robot to cover a large range of motion, it also facilitates the therapy of a wide range of patients.

\section{Redundancy}

Another key feature of ViGRR is its redundant design which allows the end-effector of the robot to reach a single point in space with multiple joint configurations. If used effectively, this redundancy can be used to find an optimal joint configuration for the task at hand. Also, the redundant design allows ViGRR to produce high forces at the end-effector without running the risk of nearing a singularity. 


\section{Low Inertia}

Compared to other lower limb rehabilitation robots, ViGRR benefits from low inertia at the end-effector. The relatively low inertia associated with the end-effector allows ViGGR to provide highly transparent control strategies.

\subsubsection{ViGRR 1.0}

From the concept design, a prototype platform was manufactured consisting of a single 4link robot for use with the patient's right leg and can be seen in Figure 2.3. This prototype is the first iteration of ViGRR and is labeled ViGRR 1.0.

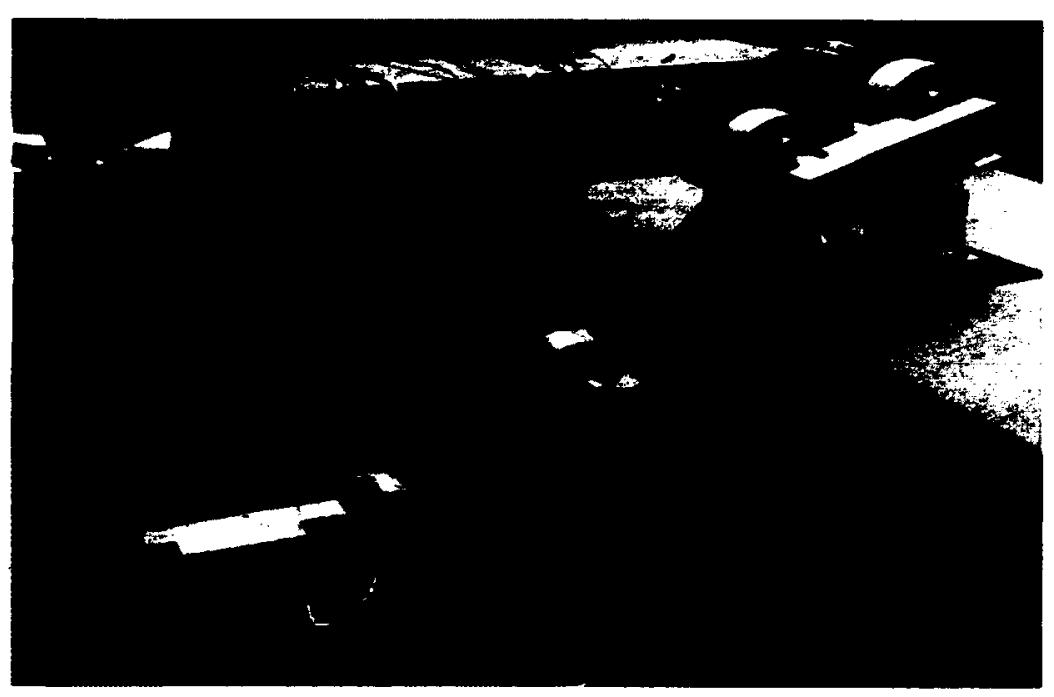

Figure 2.3: ViGRR 1.0 prototype.

\section{Hardware}

ViGRR was designed to handle ground reaction forces normally seen through walking. For this purpose, the robot links were manufactured out of 6061 T6 Aluminum "I" bar to increase bending and torsional stiffness. The motors chosen for the robot are Danaher AKM 42J, $43 \mathrm{~K}$, and $22 \mathrm{E}$. These were sized based on the maximum loads that will be delivered to the patient. Harmonic drives were implemented as a natural choice for this application due to their low backlash and compact size. The specific motor, motor drive, and harmonic drive 
selected for each link can be seen in Table 2.1 below. The Danaher motor specifications can be seen in Table 2.2 whereas the Harmonic Drive parameters are detailed in Table 2.3.

Table 2.1: ViGRR Drive Components.

\begin{tabular}{c|ccc}
\hline Joint & Motor & Harmonic Drive & S200 Drive \\
\hline \hline 1 & AKM42J-ACCNC-00 & CSF-32-120 & S21260-TVS \\
2 & AKM43K-ACCNC-00 & CSF-32-120 & S21260-VTS \\
3 & AKM22E-ACCNC-00 & CSG-25-160 & S20360-VTS \\
4 & AKM22E-ACCNC-00 & CSG-20-100 & S20360-VTS \\
\hline
\end{tabular}

Table 2.2: Danaher Motor Specifications.

\begin{tabular}{c|cccc}
\hline & Units & AKM22E & AKM42 & AKM43 \\
\hline \hline Peak Torque & $\mathrm{Nm}$ & 2.42 & 11.6 & 16.3 \\
Max Speed & $\mathrm{rpm}$ & 5410 & 6000 & 6000 \\
Peak Current & $\mathrm{A}_{R M S}$ & 9 & 33.7 & 38.3 \\
Continuous Rated & $\mathrm{Nm}$ & 0.81 & 2.38 & 2.62 \\
Rated Speed & $\mathrm{rpm}$ & 3500 & 6000 & 6000 \\
Rated Power & $\mathrm{kW}$ & 0.30 & 1.5 & 1.65 \\
Torque Constant & $\mathrm{Nm} / \mathrm{A}_{R M S}$ & 0.32 & 0.43 & 0.52 \\
Back EMF Constant & $\mathrm{V} / \mathrm{k}_{r p m}$ & 20.4 & 27.5 & 33.2 \\
Resistance & $\Omega$ & 5.09 & 0.80 & 0.70 \\
Inductance & $\mathrm{mH}$ & 9.7 & 3.1 & 2.9 \\
Inertia & $\mathrm{kgm}$ & $0.161 \times 10^{-4}$ & $1.5 \times 10^{-4}$ & $2.1 \times 10^{-4}$ \\
Weight & $\mathrm{kg}$ & 1.1 & 3.39 & 4.35 \\
Static Friction & $\mathrm{Nm}$ & 0.005 & 0.026 & 0.038 \\
Viscous Damping & $\mathrm{Nm} / \mathrm{k}_{r p m}$ & 0.0055 & 0.013 & 0.017 \\
\hline
\end{tabular}

The only contact between patient and robot is at the end-effector of the robot. The end-effector consists of a foot plate rigidly fixed to an ATI Industrial Automation 6 axis force/torque (FT) sensor as can be seen in Figure 2.4. This sensor measures the forces and torques from the patient's foot in order to control the robot. The ATI sensor was chosen since it is capable of measuring the high loads that are associated with ground reaction forces. The specifications for this sensor can be seen in Table 2.4 below. Coupled to the 
Table 2.3: Harmonic Drive Specifications.

\begin{tabular}{c|cccc}
\hline & Units & CSF-32-120 & CSG-25-160 & CSG-20-100 \\
\hline \hline Rated Torque at 2000rpm & $\mathrm{Nm}$ & 137 & 87 & 52 \\
Repeated Peak Torque & $\mathrm{rpm}$ & 353 & 229 & 107 \\
Average Torque & $\mathrm{A}_{R M S}$ & 216 & 140 & 64 \\
Momentary Peak Torque & $\mathrm{Nm}$ & 686 & 408 & 191 \\
Maximum Input Speed & $\mathrm{rpm}$ & 4800 & 5600 & 6500 \\
Average Input Speed & $\mathrm{rpm}$ & 3500 & 3500 & 3500 \\
Moment of Inertia & $\mathrm{kgm}^{2}$ & $1.69 \times 10^{-4}$ & $0.413 \times 10^{-4}$ & $0.193 \times 10^{-4}$ \\
\hline
\end{tabular}

FT sensor is the foot plate of ViGRR which attaches to the patient's foot with velcro straps. The foot plate is designed with a series of springs and an electromagnet which act as redundant safety mechanisms to detach the foot plate in the event of an emergency. This foot plate is discussed in more detail in the next section.

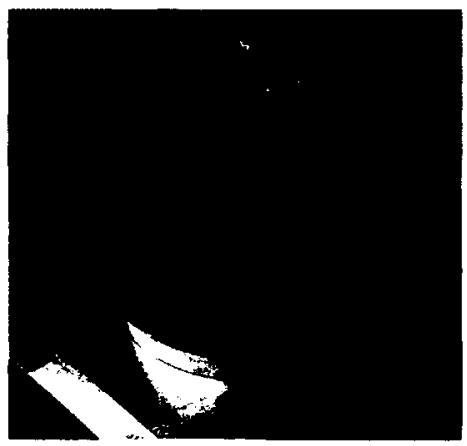

Figure 2.4: The ATI Industrial Automation 6 axis force/torque sensor mounted on the ViGRR end-effector.

Table 2.4: Force Torque Sensor Specifications.

\begin{tabular}{c|cccc}
\hline & Fx, Fy (N) & Fz (N) & Tx, Ty (Nm) & Tz (Nm) \\
\hline \hline Load Capacity & 1900 & 3800 & 80 & 90 \\
Resolution & $1 / 3$ & $5 / 12$ & $9 / 700$ & $2 / 350$ \\
Error (\%) & 1.5 & 1.0 & 1.25 & 1.25 \\
\hline
\end{tabular}




\section{Software}

The software for the ViGRR platform is developed using Simulink in conjunction with Quarc and $\mathrm{C}++$ modules on a Windows host computer. This code is converted to $\mathrm{C}++$ and downloaded to a hard real-time target computer running QNX 6.5 at a loop rate of $2 \mathrm{kHz}$. The motor drives, limit switches, and emergency stops are interfaced to the target computer using a Q8 data acquisition card (DAQ) from Quanser.

Due to the redundant design of the robot, at any given configuration there are multiple solutions for the inverse kinematics. A redundancy resolution scheme is developed for ViGRR which attempts to find an optimal robot configuration based on three objective functions. These objective functions are used to avoid joint limits, singularities, and incompatible configurations. All task space controllers for ViGRR must utilize the redundancy resolution scheme in order to avoid dangerous configurations. This redundancy resolution scheme is described in detail in [47].

Several device controllers have also been developed for ViGRR 1.0. Of particular note, an admittance controller is developed which generates a trajectory based on the force input of the user. The mass, spring, and damping parameters of the controller can be modified to produce a desired dynamic behaviour at the end-effector. A complete description of the admittance control scheme will be covered in Chapter 3.

\section{Safety}

Both hardware and software safety features have been implemented on ViGRR 1.0 to ensure that patient safety is paramount. Software safety features are defined as features which are dependant on the functionality of the real-time PC whereas hardware features are independant of the controller or PC.

Hall effect proximity sensors have been installed on all 4 links to prevent the robot from colliding with its previous link. In the event of a joint limit trigger, these sensors send a command to the DAQ card which then outputs a hold command to the motor drives. The VIGRR prototype also makes use of emergency stop buttons which, when pressed, are 
handled in the same style as the hall effect sensors. Safety limits such as position, velocity, forces and torques are also programmed into the controller to prevent the robot from unsafe behaviour.

Only one hardware safety mechanism has been implemented on ViGRR 1.0. The foot plate, which couples the patient foot to the end-effector is designed with a parallel connection of springs and an electromagnet. The springs are preloaded to detach at a specified force input, whereas the current through the electromagnet is controlled by the real-time computer. Depending on the safety situation that is being triggered, the electromagnet will either release or hold. In certain situations it is beneficial to have the foot plate held to the end-effector so as to not harm the patient.

Table 2.5 outlines all safety features which are present in ViGRR 1.0.

Table 2.5: Safety Features for ViGRR 1.0 .

\begin{tabular}{|c|c|c|}
\hline \multicolumn{3}{|c|}{ Software Limits } \\
\hline Type & Variable & Limits \\
\hline \multirow[t]{4}{*}{ Position } & $q_{1}, q_{2}, q_{3}, q_{4}$ (rads) & {$\left[\begin{array}{llll}3.28 & 2.23 & 2.67 & 2.22\end{array}\right]$} \\
\hline & & {$\left[\begin{array}{llll}-0.25 & -2.26 & -2.67 & -2.35\end{array}\right]$} \\
\hline & $X, Y, \phi(\mathrm{m}, \mathrm{m}, \mathrm{rads})$ & {$\left[\begin{array}{lll}1.5 & 2 & 3.5\end{array}\right]$} \\
\hline & & {$\left[\begin{array}{lll}-1.5 & -0.6 & -2\end{array}\right]$} \\
\hline \multirow[t]{4}{*}{ Velocity } & $\dot{q}_{1}, \dot{q}_{2}, \dot{q}_{3}, \dot{q}_{4}(\mathrm{rads} / \mathrm{s})$ & {$\left[\begin{array}{llll}2.7 & 2.5 & 3 & 3\end{array}\right]$} \\
\hline & & {$\left[\begin{array}{llll}-2.2 & -2.5 & -3 & -3\end{array}\right]$} \\
\hline & $\dot{X}, \dot{Y}, \dot{\phi}(\mathrm{m} / \mathrm{s}, \mathrm{m} / \mathrm{s}, \mathrm{rads} / \mathrm{s})$ & {$\left[\begin{array}{lll}3 & 2.5 & 4.5\end{array}\right]$} \\
\hline & & {$\left[\begin{array}{lll}-3 & -2.5 & -5\end{array}\right]$} \\
\hline \multirow[t]{2}{*}{ Torque } & $\tau_{1}, \tau_{2}, \tau_{3}, \tau_{4}(\mathrm{Nm})$ & {$\left[\begin{array}{llll}240 & 200 & 100 & 80\end{array}\right]$} \\
\hline & & {$\left[\begin{array}{llll}-240 & -200 & -100 & -80\end{array}\right]$} \\
\hline \multirow[t]{2}{*}{ Force Limits } & $F_{X}, F_{Y}, T_{\phi}(\mathrm{N}, \mathrm{N}, \mathrm{Nm})$ & {$\left[\begin{array}{lll}300 & 300 & 50\end{array}\right]$} \\
\hline & & {$\left[\begin{array}{llll}-300 & -300 & -50\end{array}\right]$} \\
\hline Hall Effect Limits & N/A & N/A \\
\hline Emergency Stops & N/A & $N / A$ \\
\hline \multicolumn{3}{|c|}{ Hardware Limits } \\
\hline Magnetic EE Release & $\mathrm{N} / \mathrm{A}$ & $\mathrm{N} / \mathrm{A}$ \\
\hline
\end{tabular}

A block diagram of the hardware, software, and safety interface on ViGRR 1.0 can be 
seen in Figure 2.5 below. For a more complete description of the platform and motivation, as well as a detailed account of the material selection and components, refer to [48] and [47].

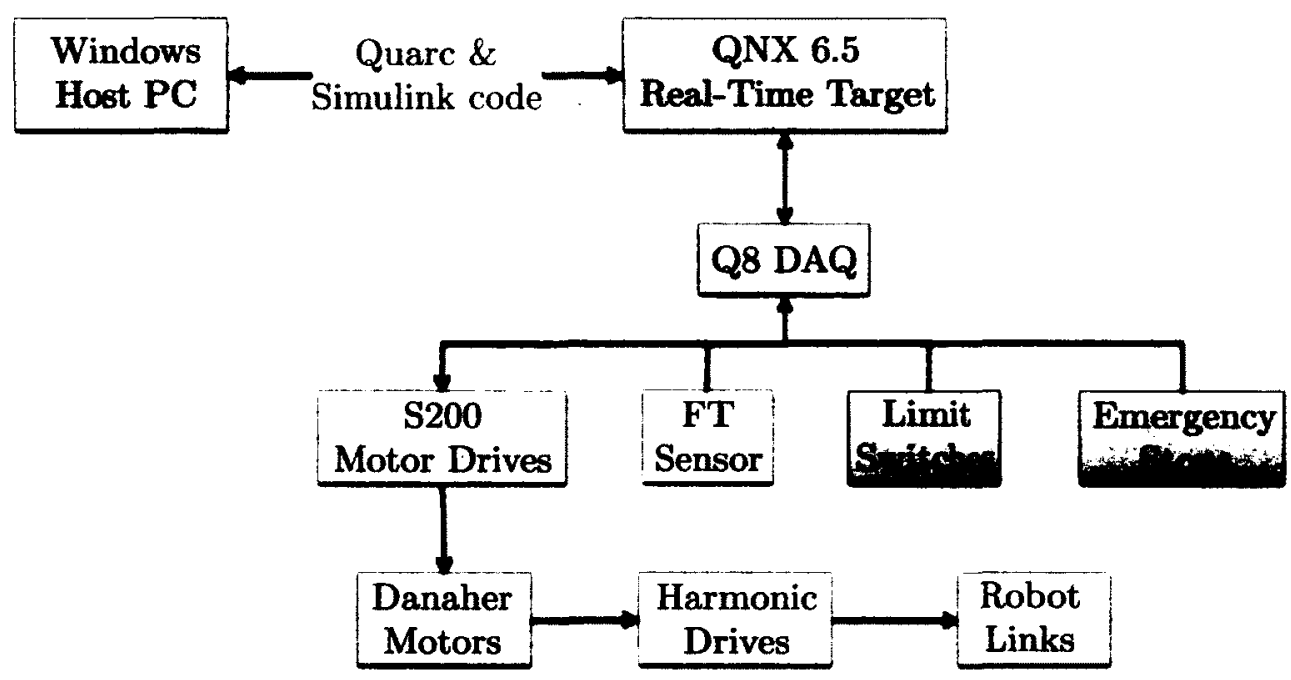

Figure 2.5: ViGRR interface block diagram.

\section{Ethics Approval}

ViGRR must be certified by the Carleton University Research Ethics Board in order to conduct experiments with human subjects. The board found that ViGRR met appropriate ethical standards as outlined in the Tri-Council Policy Statement: Ethical Conduct for Research Involving Humans, $2^{\text {nd }}$ edition and the Carleton University Policies and Procedures for the Ethical Conduct of Research. A copy of the clearance form is provided in Appendix A for reference.

\subsubsection{Required Design Enhancements for ViGRR 1.0}

The initial prototype of ViGRR, along with preliminary experiments proves that the design is functional as a robotic device. With that said, there are several shortcomings which must be addressed in order for this device to function as a haptic rehabilitation platform. The issues listed below will be addressed in the following sections. 


\section{Base Platform}

The base platform which ViGRR 1.0 is mounted on consists of a sheet of 1" thick MDF bolted to a particle board desk. Although during preliminary experiments the base platform was able to withstand the forces provided from the robot, the max load and rigidity was deemed unsuitable if loaded with a user and second robot.

\section{Patient Seat}

One of the design specifications from the concept includes a seat for the patient. A patient seat was not included in ViGRR 1.0 and must be added in order to operate the device with a lower limb.

\section{Hard Limit Switches}

Aside from the magnetic end-effector detachment, all safety protocols that are implemented on ViGRR 1.0 are handled through software. What happens if there is a problem with the software? Limit switches which can trigger without the use of software must be integrated in order to handle all failure situations.

\section{Kinematic Calibration}

Although a kinematic calibration has been performed on ViGRR 1.0, access to an Optotrak 3D positioning system has enabled a more accurate estimate of the link lengths.

\section{Foot Plate Design}

The foot plate is one of the most important hardware designs on ViGRR since it is the only point of contact between the robot and the user. The initial foot plate on ViGRR 1.0 was developed in order to test various safety mechanisms and releases. It was decided to redesign the footplate as the initial design proved to be too heavy and unreliable. 


\subsection{Base Platform}

The design requirements for the base platform consist of two items. The first is that the platform must be large enough to mount two robots and a patient seat. Second, the base platform must be able to withstand the static and dynamic loading associated with two robots, a seat, and a user. The material chosen for the base platform is a sheet of $30 " \times 60 " \times \frac{1}{4} "$ steel for the top and $3 " \times 3 " \times \frac{1}{4} "$ steel for the posts. Several worst case scenario loading conditions were tested on the CAD model through FEA analysis. The worst case scenario was chosen to be with two robots fully extended and providing ground reaction forces of $950 \mathrm{~N}$ to each leg. The total loading condition with a safety factor of 3 can be seen in Table 2.6. Figure 2.6 shows the FEA of the maximum loading condition. With this load, a deflection of $0.3369 \mathrm{~mm}$ can be seen at the centre of the platform.

Table 2.6: Base Platform Maximum Loading Condition for FEA.

\begin{tabular}{c|c|c}
\hline \hline Load Source & Value & Load after S.F. 3 \\
\hline Ground Reaction Force (X) & $950 \mathrm{~N}$ & $2850 \mathrm{~N}$ \\
Ground Reaction Force (Y) & $220 \mathrm{~N}$ & $660 \mathrm{~N}$ \\
Ground Reaction Moment & $242 \mathrm{Nm}$ & $726 \mathrm{Nm}$ \\
Occupant & $981 \mathrm{~N}$ & $2943 \mathrm{~N}$ \\
\hline
\end{tabular}

\subsection{Patient Seat}

The patient seat for ViGRR was designed and built by an undergraduate student, Daniel Goubran, with the author's guidance. The seat was designed to be adjustable from 0 to $90^{\circ}$, allow for unhindered leg movement, and be able to slide along the direction of the patient's torso. Figure 2.7 shows the side view of the patient seat.

\subsection{Hard Limit Switches}

The addition of new limit switches motivated the acquisition of a more compact sensor. Therefore, all limit switches for the ViGRR robot were upgraded from non-latching single 


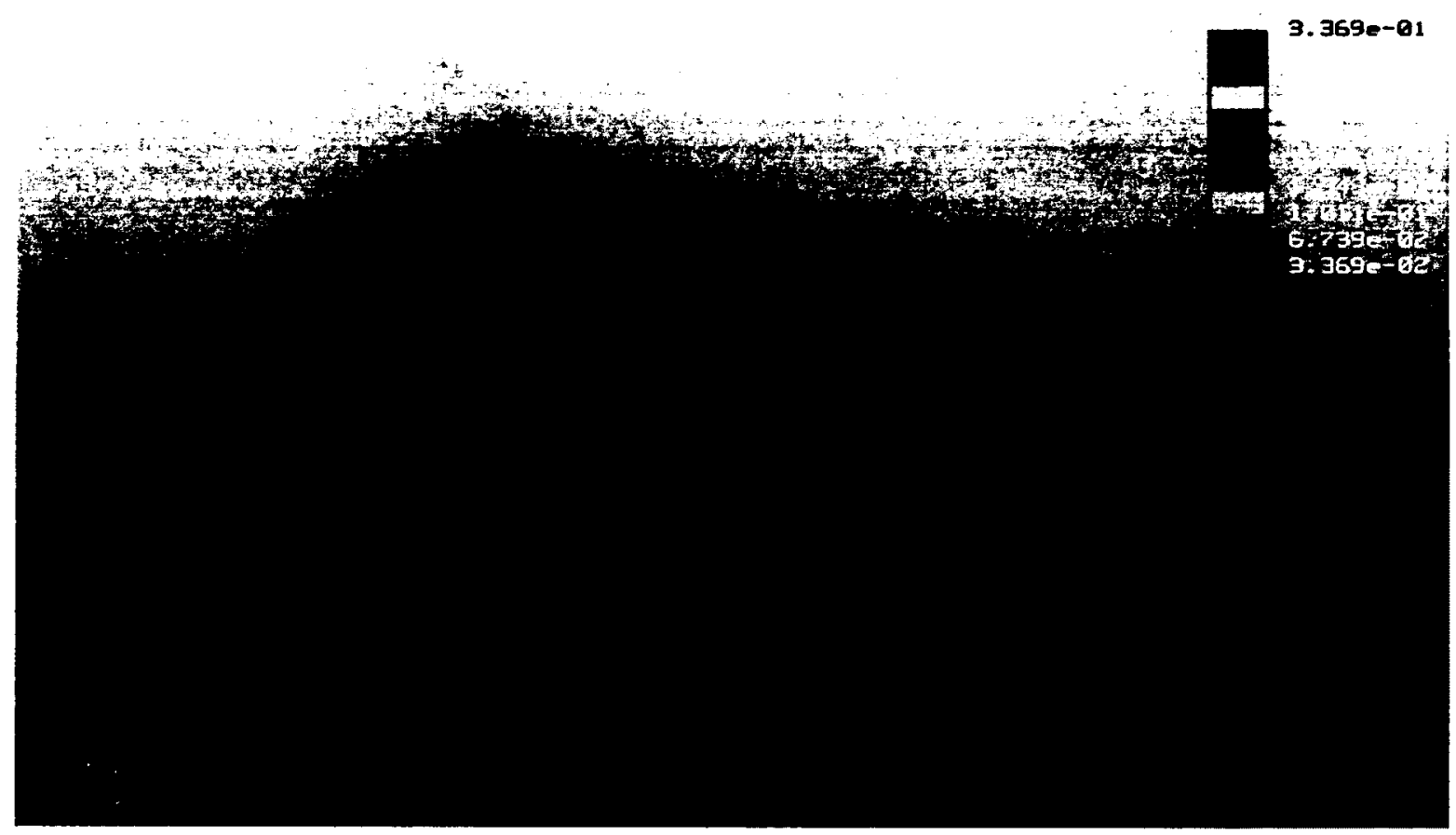

Figure 2.6: FEA analysis of the base platform.

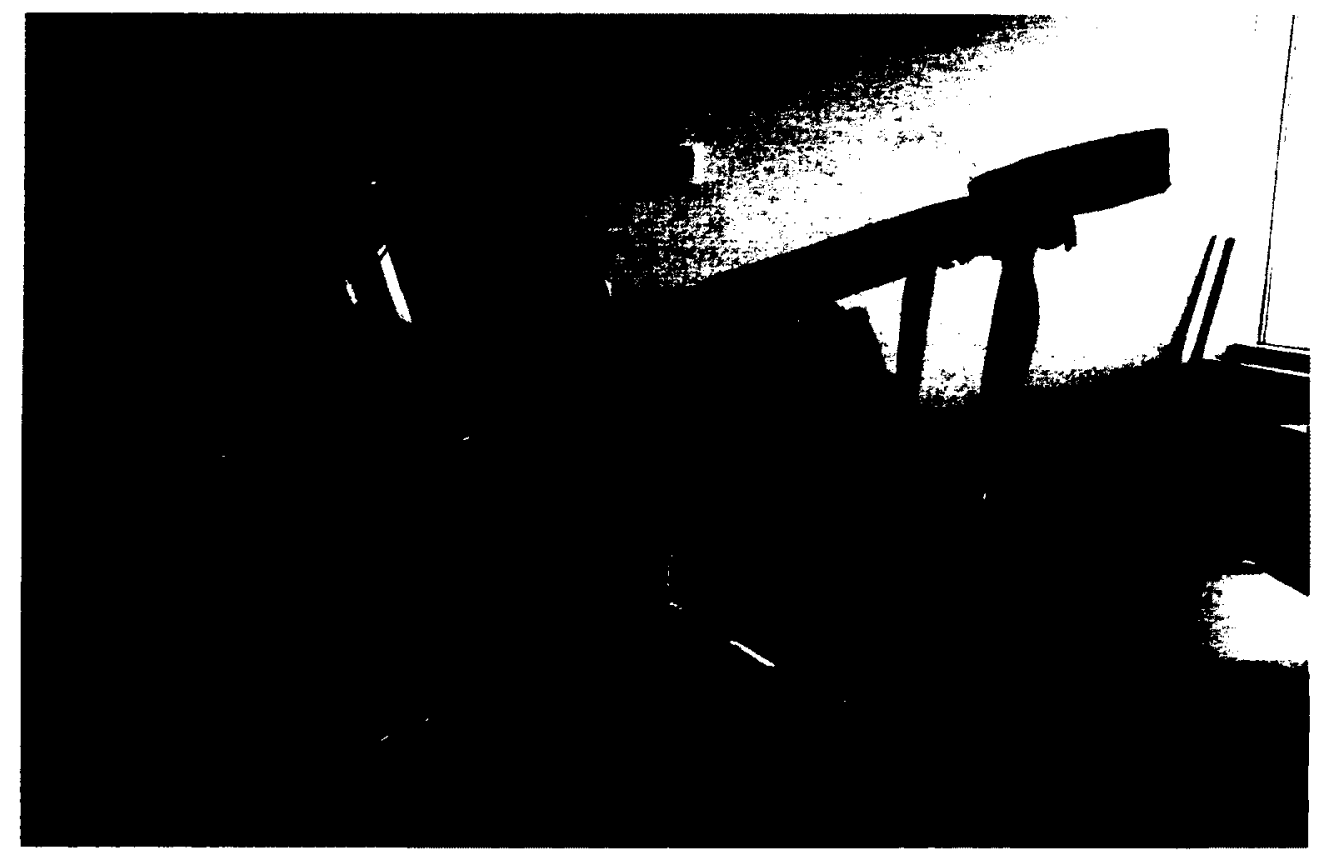

Figure 2.7: Side view of the patient seat. 
pole Hall effect proximity sensors by Infineon Technologies to D-F9P solid state proximity sensors from SMC (Figure 2.8).

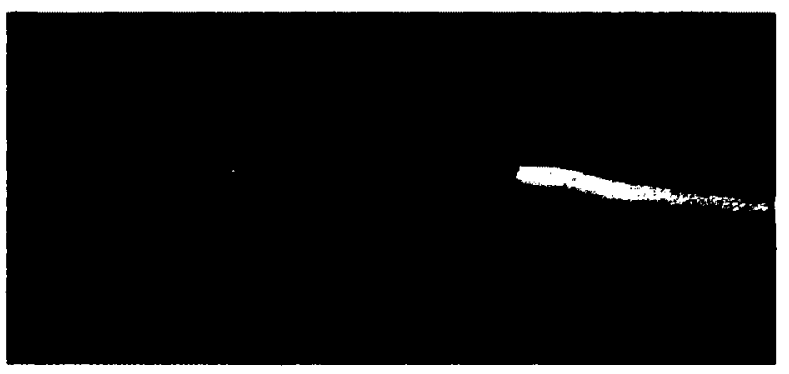

Figure 2.8: D-F9P solid state proximity sensor from SMC.

Previously there existed 8 limit switches on the robot, one for the positive and negative rotation of each link. In order to incorporate redundant safety limits, the number of limit switches has been extended to 16. Each link now has a soft limit that will trigger first, sending a signal to the controller to hold that motor. If the robot fails to hold or an error occurs, the link will continue to rotate until it hits the second hard limit. This hard limit is wired to the drive unit of the motor which prevents the motor from rotating in that direction. Figure 2.9 below shows the placement of each limit switch.

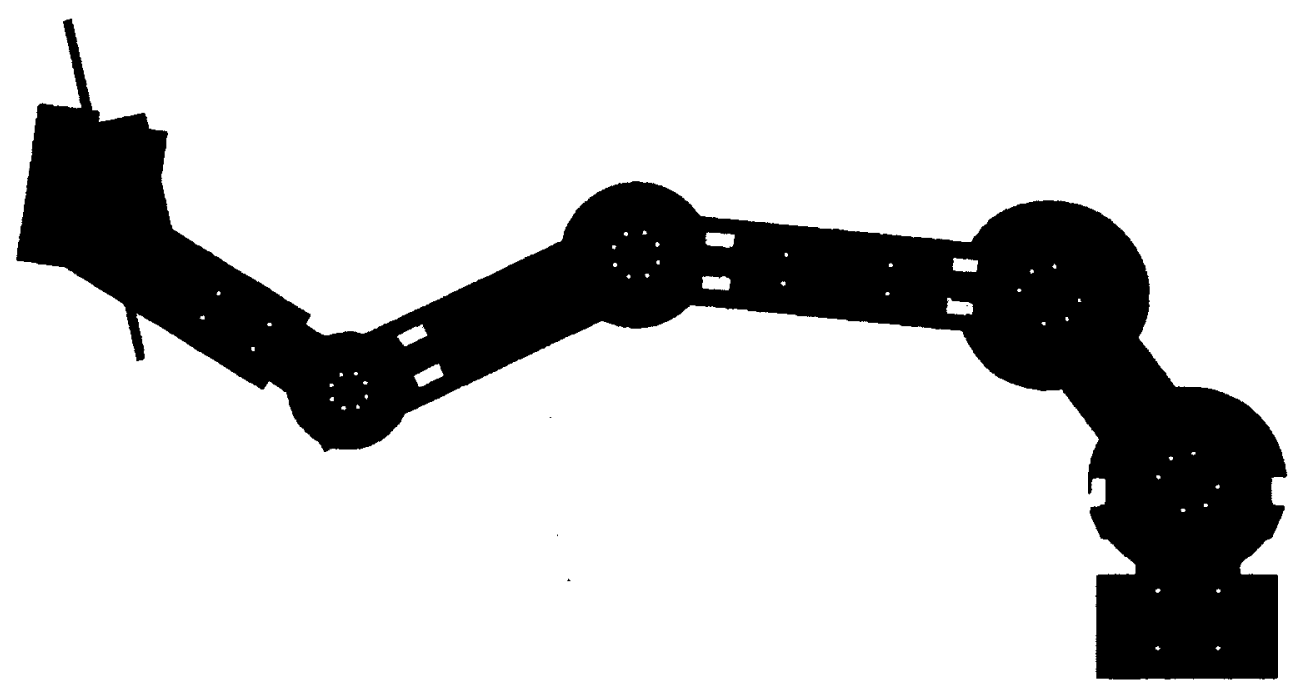

Figure 2.9: Limit switch configuration on ViGRR. The yellow blocks represent the soft limits and black blocks represent the hard limits. 


\subsection{Interface Board}

An interface board was designed to connect the data acquisition card to the motor drives, limit switches, and electromagnets. This interface board manages all signal routing and simplifies the external wiring needed to control the ViGRR platform. The interface board was designed with the help of a PhD candidate Kyle Chisholm in EAGLE 5.6.0 design software. The design was sent out for fabrication to Batch PCB and is shown in Figure 2.10. The EAGLE schematic and circuit board views are located in Appendix B. The main components of the board are highlighted in Figure 2.10 and are described below.

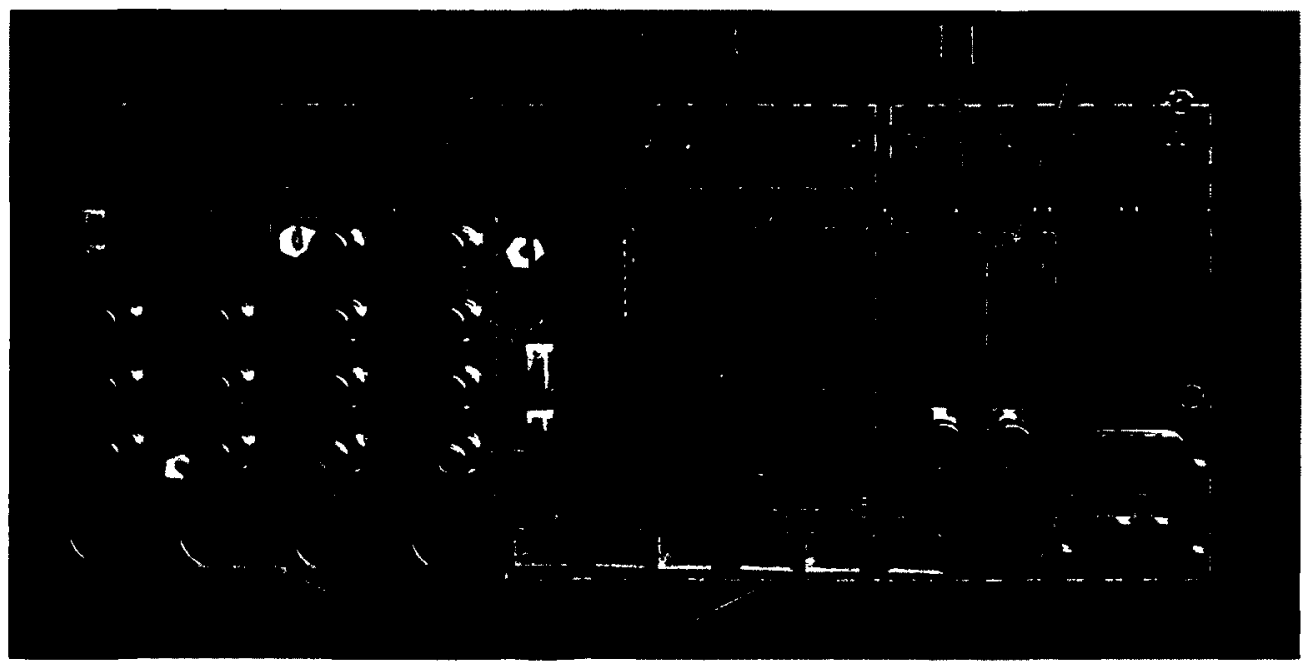

Figure 2.10: Interface Board PCB.

\section{S200 Motor Drive Interface}

1. 26 pin D-Sub inputs from the $\mathrm{S} 200$ motor drives. There are 4 connectors located on the back of the PCB to interface to each motor drive.

2. 4 Single ended encoder outputs to the DAQ card.

3. 65LBC175A differential line receivers. Their purpose is to change the encoder outputs from differential to single ended for compatibility with the Q8 DAQ card.

4. 4 Analog inputs for the torque commands to each motor drive. 
5. 2 Analog monitors per motor drive ( 8 total). The $\$ 200$ motor drives can be programmed to output various information about the specific drive through the analog monitors such as current feedback and temperature.

6. Serial communication to each motor drive. The S200 motor drives are programmed individually by interfacing a single RS-232 serial connector from the board to the PC. A switch is used to select the appropriate drive unit which routes the serial communication from the RS-232 connector to the appropriate RJ-11 connector on the back of the PCB.

\section{Board Power}

7. Isolated connectors for $+5 \mathrm{~V}$ and $+24 \mathrm{~V}$ power source. $+5 \mathrm{~V}$ power is necessary for all IC's to function and $+24 \mathrm{~V}$ provides power to the electromagnets.

8. $1 \mathrm{~A}$ fuse in series with the $+24 \mathrm{~V}$ power to prevent damage to the electromagnets.

\section{Emergency Stop}

9. Connectors for 2 emergency stops. One connector is routed through the digital I/O bank and the other is routed directly to the quad buffer which controls the motor drive enables.

10. $74 \mathrm{HC} 125 \mathrm{~N}$ quad buffer controls the state of the motor drive enables. If the emergency switch is pressed, the buffer disables the motors.

\section{Footplate Electromagnet Interface}

11. Connectors to the footplate electromagnets.

12. Analog voltage input to control the electromagnet holding force.

\section{Optotrak Interface}


13. Synchronization interface to the Optotrak Certus camera system

\section{Digital I/O}

14. 32 Digital I/O's for interfacing with the Q8 DAQ card.

\section{Limit Switch Interface}

15. Connectors for the 16 limit switches attached to the robot.

16. The Q8 DAQ card requires sourcing external inputs, however the D-F9P hall effect sensors are sinking type. 4, MMPQ3904 transistor banks are necessary to convert the limit switches to sourcing type. The schematic for the circuit is provided in Appendix B.

\subsection{Kinematic Calibration}

Kinematic calibration of the ViGRR robot is necessary in order to ensure that the endeffector pose can be controlled as accurately as possible. The link lengths of the robot were estimated using a least squares method which requires simultaneous measurements of both the link angles and end-effector pose. The link angles of the robot can be measured using the motor encoders whereas the end-effector pose can be measured by constraining the end-effector pose to a position of known precision and allow the links to move freely. Alternatively, the end-effector pose can be measured with an external sensor during robot motion. Access to an accurate camera measurement system motivated the use of the latter technique.

\subsubsection{Coordinate Frames and the Optotrak Motion Capture System}

The Optotrak Certus Motion Capture System by NDI is a 3D camera measurement system which collects data using optical markers with an accuracy of $0.1 \mathrm{~mm}$. Three markers 
can be combined to create a rigid body which will then allow the data to be output as a homogenous transformation matrix. Both the rigid body marker and Optotrak camera system can be seen in Figure 2.11 below. All marker data is collected with respect to the Optotrak camera frame $\{P\}$. The coordinate frames of the robot links were defined using the Optotrak in order to transform the marker data to the ViGRR origin.

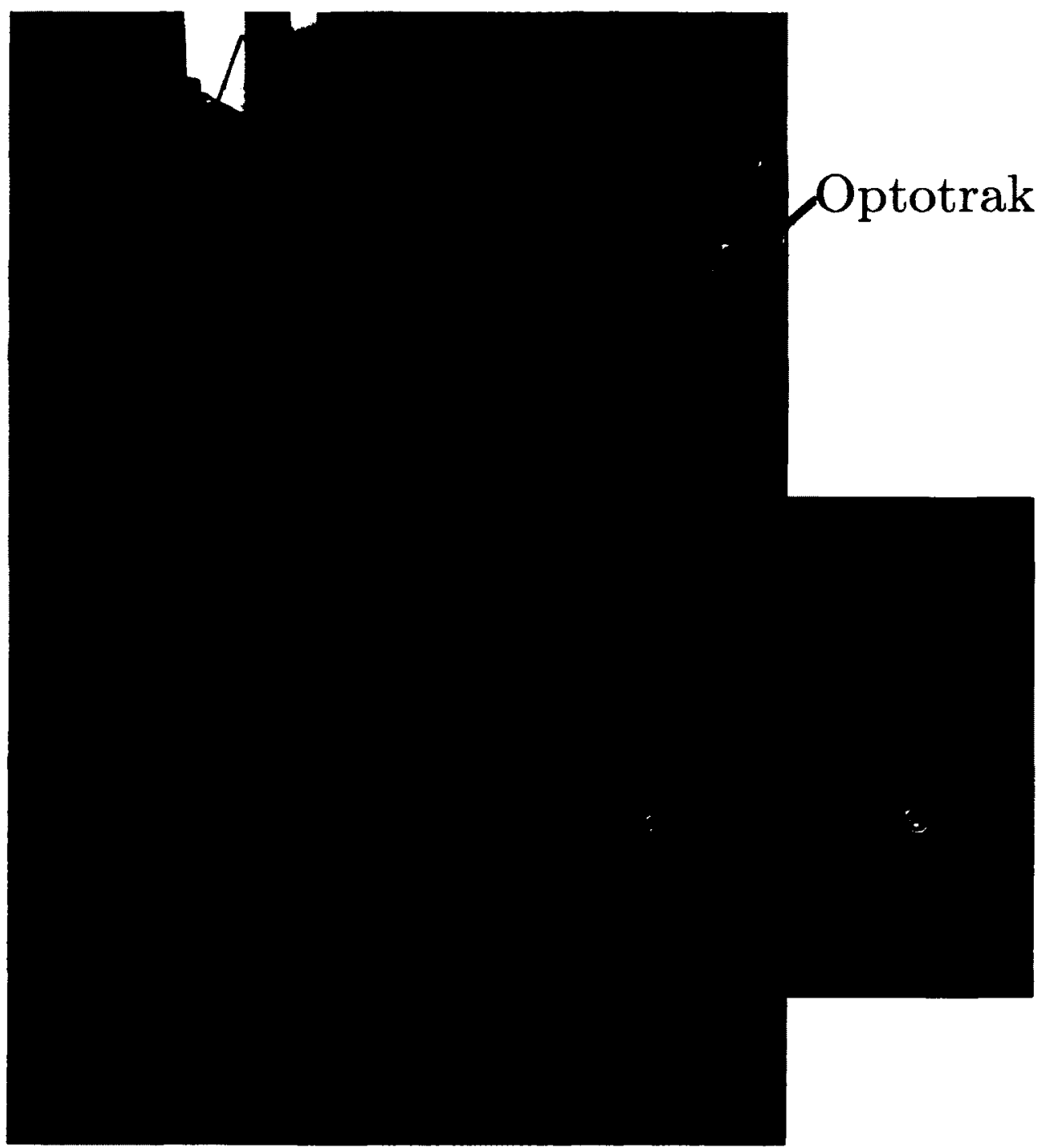

Figure 2.11: Optotrak Certus Motion Capture System and rigid body marker by NDI.

The diagram in Figure 2.12 displays all of the robot coordinate frames. The origin of ViGRR, labeled $\{0\}$, is attached to the centre of rotation of the first link with the $Z$ axis pointing into the motor and the $X$ axis aligned with the horizontal. Frames $\{1\}$ through 
$\{3\}$ are attached to their respective links at their centre of rotation with the same $Z$ axis convention and their $X$ axes aligned with the subsequent frame. Frame $\{4\}$ is similar to the previous 3 frames except that its $X$ axis is aligned through the centre of the FT sensor. The end-effector coordinate frame is defined as $\{E E\}$ with its origin at the centre of the FT sensor and is aligned with frame $\{4\}$. The FT sensor has its own coordinate frame located on its surface which is labelled $\{F T\}$. Also, the foot plate has a coordinate frame associated with it which is labelled $\{F P\}$ and is located at the back of the footrest with its $X$ axis aligned with its surface.

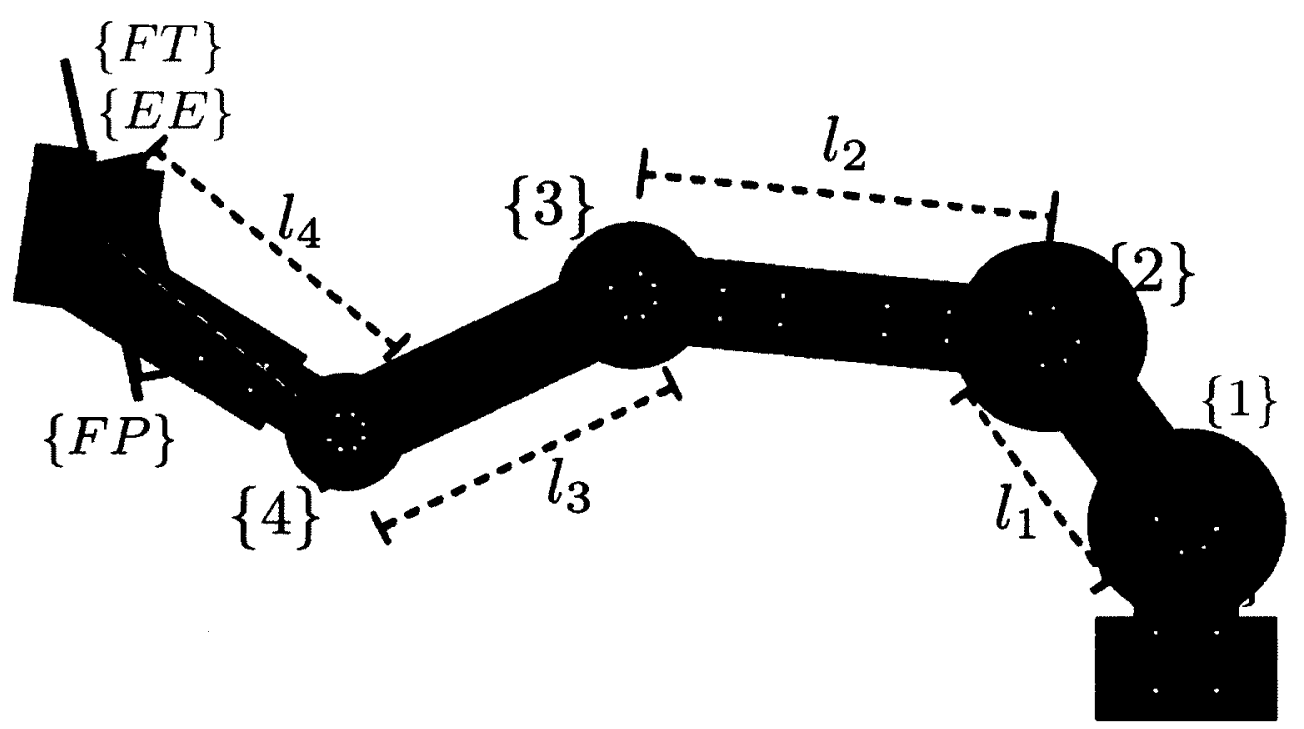

Figure 2.12: Coordinate frames of ViGRR.

Data for the kinematic calibration was collected by placing rigid body markers on both the footplate and the base platform as seen in Figure 2.13. The data collected would give information about the rigid transformation of the markers $\{O F P\}$ and $\{b\}$ with respect to the camera frame $\{P\}$. The rigid body markers $\{O F P\}$ and $\{b\}$ are stationary with respect to ViGRR frames $\{F P\}$ and $\{0\}$ respectively. Therefore, static transformations ${ }^{0} T_{b}$ and ${ }^{O F P} T_{E E}$ can be defined which will transform the Optotrak data to the ViGRR origin as follows:

$$
{ }^{0} T_{E E}={ }^{0} T_{b}{ }^{b} T_{P}{ }^{P} T_{O F P}{ }^{O F P} T_{E E}
$$




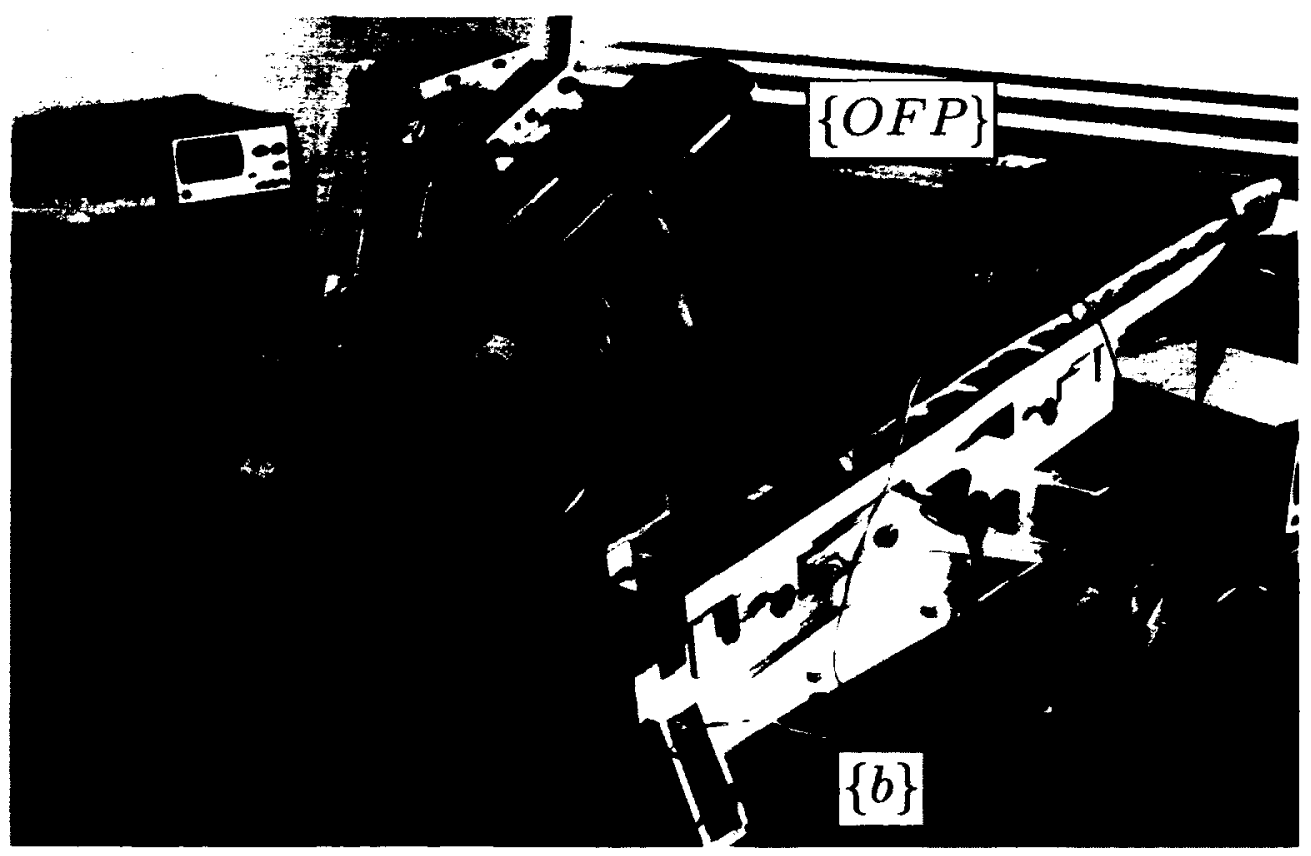

Figure 2.13: Optotrak markers placed on ViGRR. $\{O F P\}$ represents the marker frame located on the footplate and $\{b\}$ represents the marker frame located on the base platform.

\subsubsection{Link Length Estimation}

The link lengths of a planar robot can be estimated using a linear least squares method. The forward kinematics of the ViGGR can be written as

$$
\left[\begin{array}{l}
x \\
y \\
\phi
\end{array}\right]=\left[\begin{array}{c}
l_{1} \cos \left(q_{1}\right)+l_{2} \cos \left(q_{1}+q_{2}\right)+l_{3} \cos \left(q_{1}+q_{2}+q_{3}\right)+l_{4} \cos \left(q_{1}+q_{2}+q_{3}+q_{4}\right) \\
l_{1} \sin \left(q_{1}\right)+l_{2} \sin \left(q_{1}+q_{2}\right)+l_{3} \sin \left(q_{1}+q_{2}+q_{3}\right)+l_{4} \sin \left(q_{1}+q_{2}+q_{3}+q_{4}\right) \\
q_{1}+q_{2}+q_{3}+q_{4}
\end{array}\right],
$$

where $q$ and $l$ represent the link angles and the link lengths respectively. Ignoring the angle of rotation $\phi$, the link angles can be extracted from the forward kinematics to yield

$$
\left[\begin{array}{l}
x \\
y
\end{array}\right]=\left[\begin{array}{cccc}
\cos \left(q_{1}\right) & \cos \left(q_{1}+q_{2}\right) & \cos \left(q_{1}+q_{2}+q_{3}\right) & \cos \left(q_{1}+q_{2}+q_{3}+q_{4}\right) \\
\sin \left(q_{1}\right) & \sin \left(q_{1}+q_{2}\right) & \sin \left(q_{1}+q_{2}+q_{3}\right) & \sin \left(q_{1}+q_{2}+q_{3}+q_{4}\right)
\end{array}\right]\left[\begin{array}{l}
l_{1} \\
l_{2} \\
l_{3} \\
l_{4}
\end{array}\right] .
$$


The link lengths can then be estimated by

$$
\left[\begin{array}{c}
\iota_{1} \\
\iota_{2} \\
l_{3} \\
l_{4}
\end{array}\right]=\left(\left[\begin{array}{cccc}
\cos \left(q_{1}\right)_{1} & \cos \left(q_{1}+q_{2}\right)_{1} & \cos \left(q_{1}+q_{2}+q_{3}\right)_{1} & \cos \left(q_{1}+q_{2}+q_{3}+q_{4}\right)_{1} \\
\sin \left(q_{1}\right)_{1} & \sin \left(q_{1}+q_{2}\right)_{1} & \sin \left(q_{1}+q_{2}+q_{3}\right)_{1} & \sin \left(q_{1}+q_{2}+q_{3}+q_{4}\right)_{1} \\
\vdots & \vdots & \vdots & \vdots \\
\cos \left(q_{1}\right)_{n} & \cos \left(q_{1}+q_{2}\right)_{n} & \cos \left(q_{1}+q_{2}+q_{3}\right)_{n} & \cos \left(q_{1}+q_{2}+q_{3}+q_{4}\right)_{n} \\
\sin \left(q_{1}\right)_{n} & \sin \left(q_{1}+q_{2}\right)_{n} & \sin \left(q_{1}+q_{2}+q_{3}\right)_{n} & \sin \left(q_{1}+q_{2}+q_{3}+q_{4}\right)_{n}
\end{array}\right]\right)^{-1}\left[\begin{array}{c}
x_{1} \\
y_{1} \\
\vdots \\
x_{n} \\
y_{n}
\end{array}\right],
$$

where $n$ is the number of measurement points. Table 2.7 shows the previous estimated link lengths and the new estimated link lengths using the kinematic calibration described above. Link 4 shows the biggest deviation in length, however this is to be expected as the end-effector was modified between calibrations.

Table 2.7: Link Parameters from Kinematic Calibration.

\begin{tabular}{|c|c|c|}
\hline Link & Previous Estimated Length (mm) & New Estimated Length (mm) \\
\hline \hline 1 & 206.1339 & 195.2169 \\
2 & 359.7399 & 370.1169 \\
3 & 281.9941 & 270.7659 \\
4 & 319.9202 & 269.9694 \\
\hline
\end{tabular}

A sample set of data points were taken to assess the accuracy of the kinematic calibration. The robot was given a trajectory to follow for 300 seconds. Due to the varying sample rate of both the Optotrak $(100 \mathrm{~Hz})$ and QNX $(2000 \mathrm{~Hz})$, the robot would pause at a given position every 5 seconds where an average position value would be taken. The Euclidean distance between the Optotrak data and encoder data were calculated for both the previous and current estimated link lengths. This distance is defined as the error between the actual (Optotrak) and measured (encoder) data. The mean error for the previous estimated link lengths was calculated to be $10.8042 \mathrm{~mm}$ and the mean error for the new link lengths was calculated as $2.8576 \mathrm{~mm}$. The new estimate of the link lengths shows an improvement in mean error of $7.9466 \mathrm{~mm}$. It is believed that although in theory this calibration is a linear problem, there are small offsets in the axes of the motors which can cause larger deviations 
in end-effector position. Also, the flexibility in the output of the harmonic drives were not modelled and would cause inaccuracies in the forward kinematics. If another calibration is to be performed, the harmonic drive flexibility must be accounted for.

\subsection{Foot Plate Design}

As stated in the introduction, ViGRR is an end-effector type haptic robot. Unlike exoskeleton type lower limb robots whose contact with the user is at multiple points along the leg, ViGRR has only one contact point at the foot. Therefore, this contact point is an essential component of ViGRR and must be designed with strict criteria to ensure the safety of the patient.

\subsubsection{Design Requirements}

The design requirements for the foot plate are summarized in the list below and have been expanded in their corresponding sections.

\section{Holds user's foot}

A fairly obvious requirement is that the foot plate must be able to secure the foot of the user. The foot must be held in place with no effort from the user. Also, the foot plate should be adjustable for any size of shoe and allow for quick attachment to the user's foot and end-effector.

\section{Complete detachment}

As a safety measure, the foot plate must allow for complete detachment from the robot. Also, the detachment must be independant of the orientation of the foot plate.

\section{Redundant safety}

In the event of an emergency, the foot plate must have redundant safety release mechanisms to detach itself from the end-effector and prevent injury to the user. Some safety features could include springs, magnets, mechanical latches, electric solenoids, or use of a material which yields or fails at defined maximum loads. 


\section{Variable safety release}

Depending on the exercise that is being performed, the force limits that are defined for the user may be desired to vary. A requirement for the foot plate is to have the ability to detach at different limits set by the operator.

\section{Lightweight}

The foot plate will be attached to the FT sensor at the end-effector of the robot. Therefore, keeping the foot plate as light as possible is essential so that the force measurements are not corrupted with added mass and inertia effects. This effect is not avoidable, however it can be mitigated through an economic use of material.

\subsubsection{Initial Foot Plate Design}

The initial design of the foot plate was developed as a prototype to be critically evaluated and improved upon. This foot plate was designed to attach to the FT sensor on the ViGRR end-effector as seen in Figure 2.14. The prototype foot plate was developed by a previous masters student, Adam Mullins [48]. This design utilized 3 separate release mechanisms with the use of electromagnets and springs as can be seen in Figure 2.15.

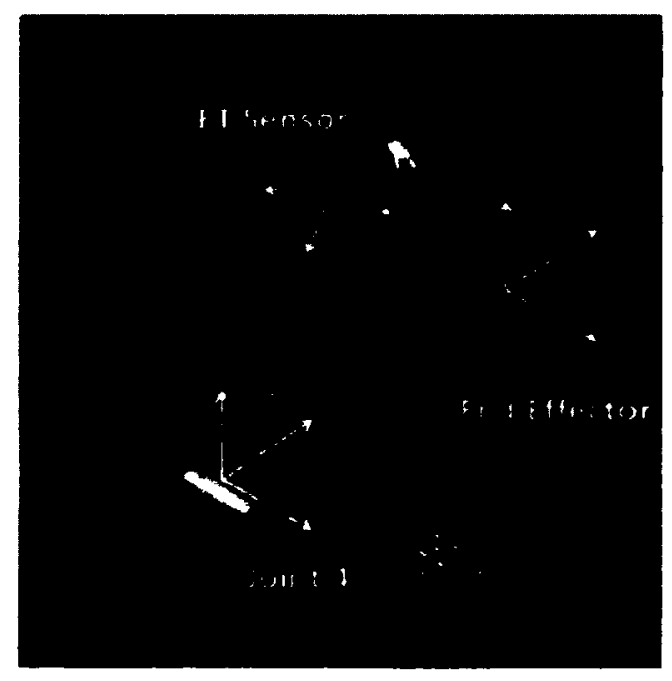

Figure 2.14: Coordinate system of the previous ViGRR end-effector. 


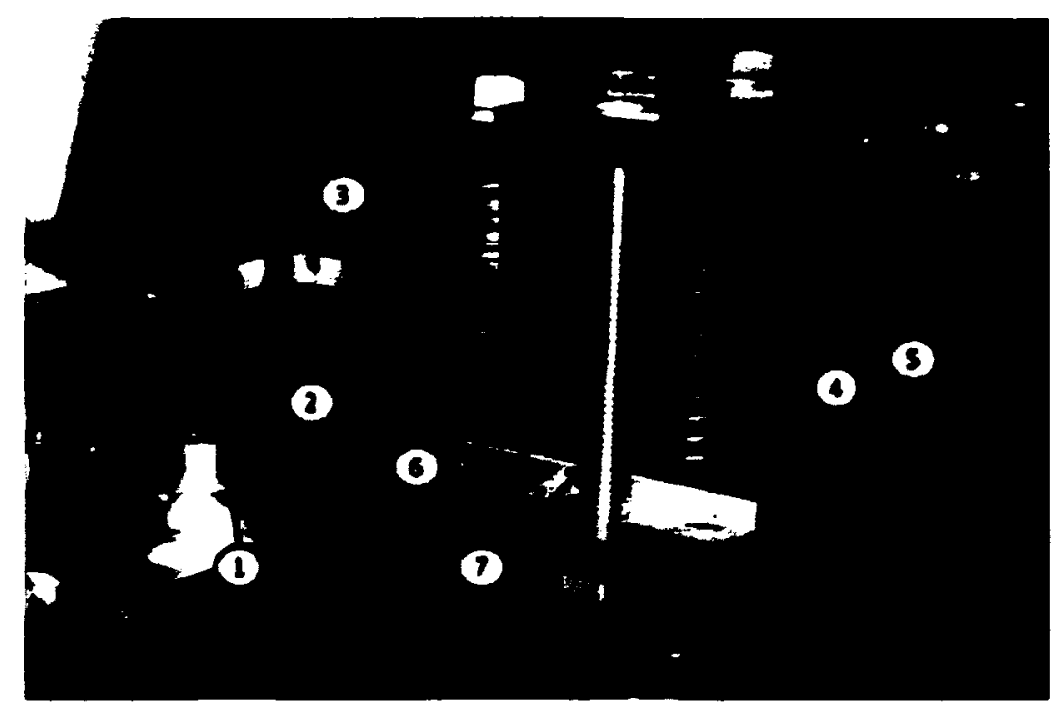

Figure 2.15: Initial design of the foot plate. 1) FT sensor. 2) Electromagnet. 3) Extension Spring. 4) Contraction Spring. 5) Foot attachment plate. 6) Guide rods. 7) Pretension screws.

\section{Electromagnet}

Electromagnets are a natural choice for the release mechanism since they provide two levels of safety. The first level of safety is that they can be controlled in software which can disengage the magnet in case of an error. The magnet will also disengage if there is a power failure. The second level of safety occurs when the patient's foot applies a greater force than the magnet can hold and the foot plate disengages.

The magnet present on this design is a Securitron MCL-24 Magnetic Lock with a 90kg holding capacity. This magnet was chosen due to its relatively high holding force to weight ratio. The magnet force is controlled through an analog voltage signal (0-24 Volts DC) which determines the amount of current through it. Although the electromagnet is mounted near the FT sensor, tests have been conducted which prove that the electromagnet has no discernible effect on the sensor measurements [48].

The electromagnet for the foot plate is controlled through an MMPQ3904 NPN transistor. From the data sheet, the MMPQ3904 has an $h_{f e}$ of 100 and a $V_{B E}$ of $1.2 \mathrm{~V}$. The circuit 
for the magnet can be seen in Figure 2.16. The base resistor $R_{b}$ was selected through

$$
R_{b}=\frac{V_{D A Q}-V_{B E}}{I_{b}}
$$

where

$$
I_{b}=\frac{I_{c}}{h_{f e}}
$$

The DAQ card outputs a maximum positive voltage of $10 \mathrm{~V}$. In order to maximize the voltage swing that can be controlled, $10 \mathrm{~V}$ will represent the transistor saturated. Since the maximum current allowable for a single magnet is $62 \mathrm{~mA}$, an $R_{b}$ of $15 k \Omega$ was chosen.

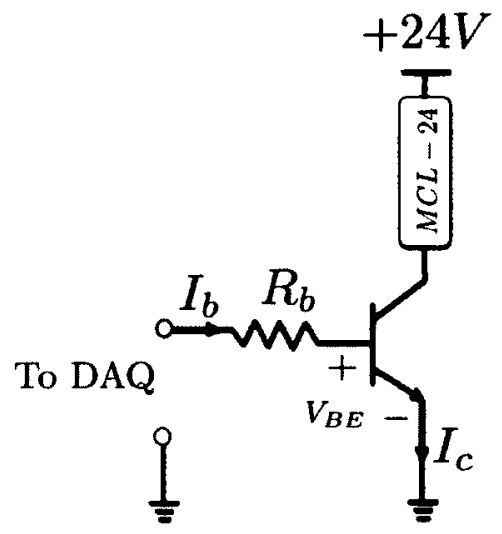

Figure 2.16: Schematic diagram of the electromagnet circuit where MCL-24 represents the electromagnet.

\section{Mechanical Spring Release}

The holding springs offer the third level of mechanical safety. The foot plate is designed to release at different forces depending on if the user is extending or contracting their leg. The springs, labelled 3 and 4 in Figure 2.15, are designed to decouple the foot plate at a higher load in compression than in tension. Initially, the pretension screws are tightened to provide an initial force threshold. As the user pushes on the top of the foot plate, both springs will need to be compressed in order to decouple the mechanism. However, if the 
user pulls on the foot plate, only the longer spring will need to be compressed for decoupling to occur.

\section{Initial Design Issues}

The relative size and weight of the initial foot plate creates a large moment arm on the magnet. The force that this moment produces on the magnet is too large for the magnet to hold the foot plate. The magnet could be rotated $90^{\circ}$, however the same problem would arise if the foot plate was torqued about its $\mathrm{Z}$ axis. The large moment arm also has a negative effect on the FT sensor. In its simplest form, the forces felt by the foot are equal to the moment measured at the sensor divided by the distance from the foot to the sensor. Therefore, the moment arm should be reduced as much as possible to reduce the relative force and moments acting on the sensor. Another issue with this design comes from a property of the electromagnet. The magnet is rated at $91 \mathrm{~kg}$ of holding force in the axial direction, however this force is substantially lower in the shear direction. The foot plate would have little resistance to slide off the magnet if shear forces were applied by the foot. For these reasons the initial design did not meet the requirements and a second design was developed which iterates on the existing prototype.

\subsubsection{Modified Foot Plate Design}

A modification of the ViGRR end-effector was needed in order for the foot plate to meet all of the criteria listed above. The initial and modified end-effector designs can be seen in Figure 2.17 below. The modified end-effector design mounts the FT Sensor at $90^{\circ}$ from its $X$ axis. This allows for the foot plate to be completely free from collision with the robot when a detachment occurs and allows the foot plate to be mounted closer to the FT sensor.

The modified foot plate design builds upon the previous design by using the electromagnet concept. The spring mechanism, however was discarded as that was deemed too heavy and complex. In order to increase the holding force and moment, this design incorporates two magnets instead of one. The modified design was developed in Autodesk Inventor and can be seen in Figs 2.18(a) and 2.18(b). 


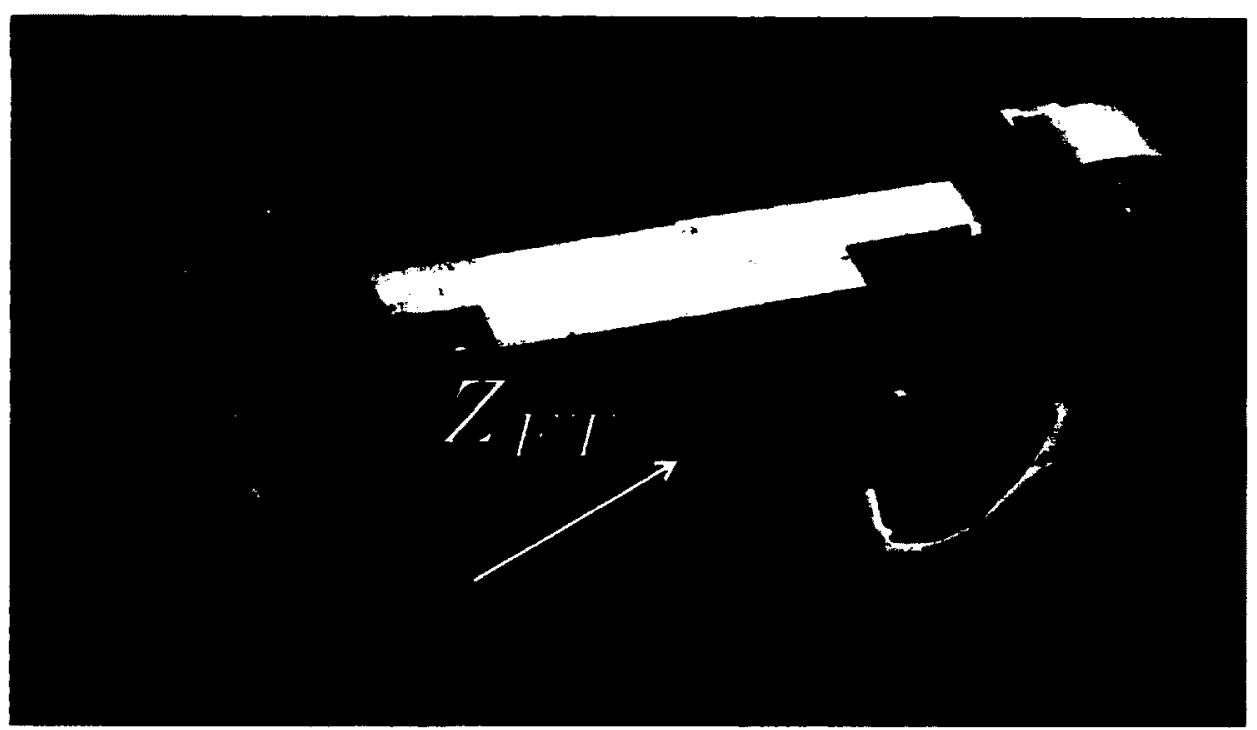

(a)

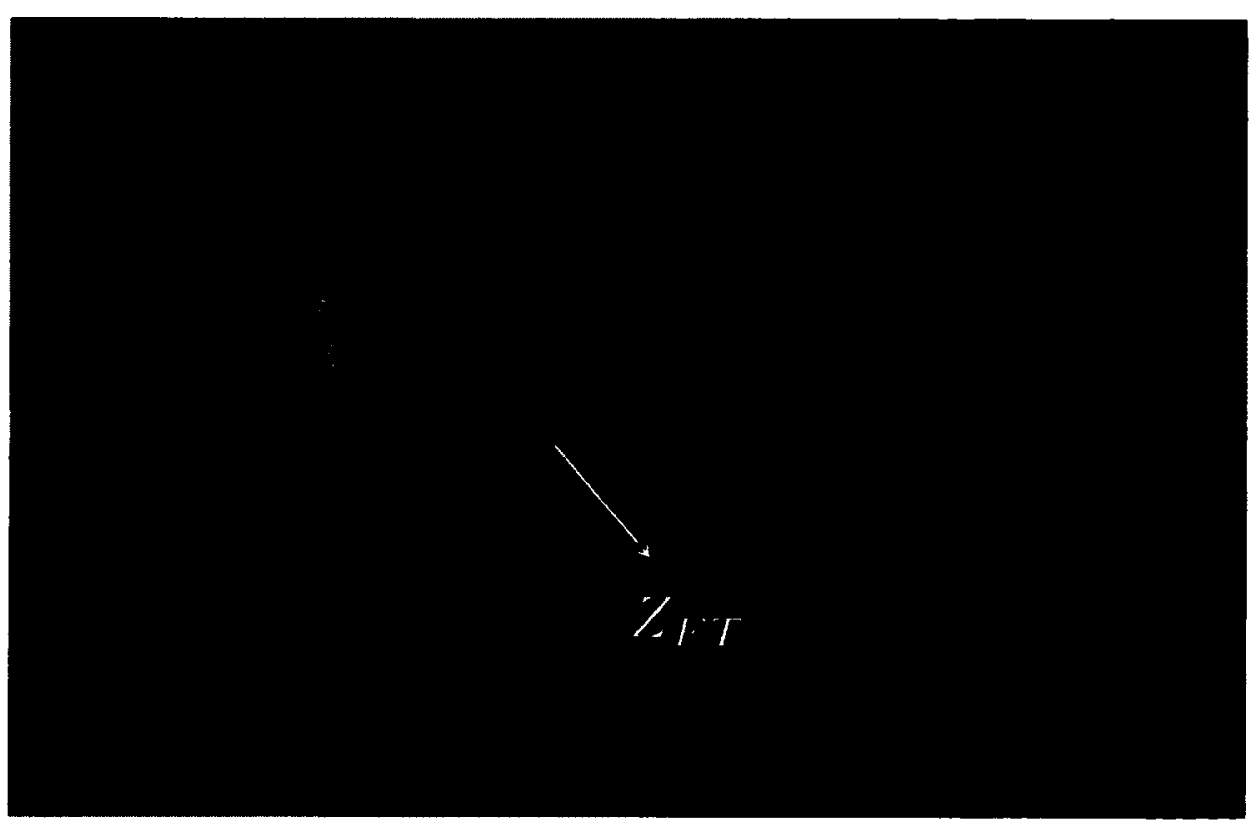

(b)

Figure 2.17: (a) Initial end-effector orientation. (b) Modified end-effector orientation. (FT sensor $Z$ axis shown). 


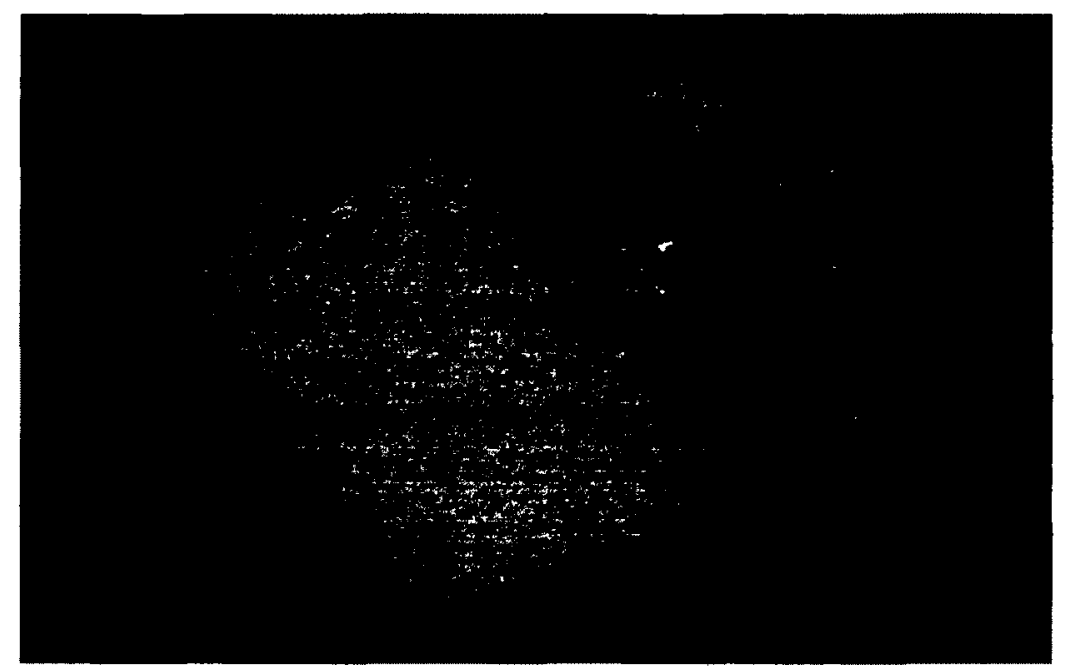

(a)

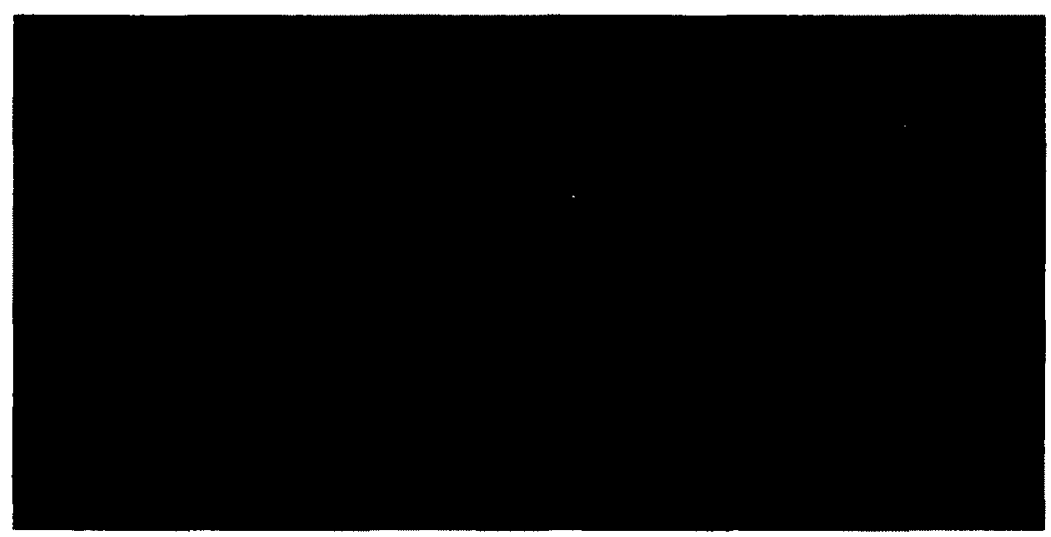

(b)

Figure 2.18: CAD model of the end-effector with magnet engaged (a) and disengaged (b). 
In this configuration, the magnets are preventing the foot plate from being detached by a force in the $\mathrm{Z}$ direction. Any forces acting in the sagittal plane will produce shear on the magnets and will cause the foot plate to detach prematurely. Therefore, two special locking cones were designed and built which would hold the foot plate from shearing and rotating. The cones on one side of the foot plate have a convex shape whereas the mating side has a concave shape (Fig 2.19). These cones are threaded and can be replaced with other cones of varying slope in order to vary the holding moments.

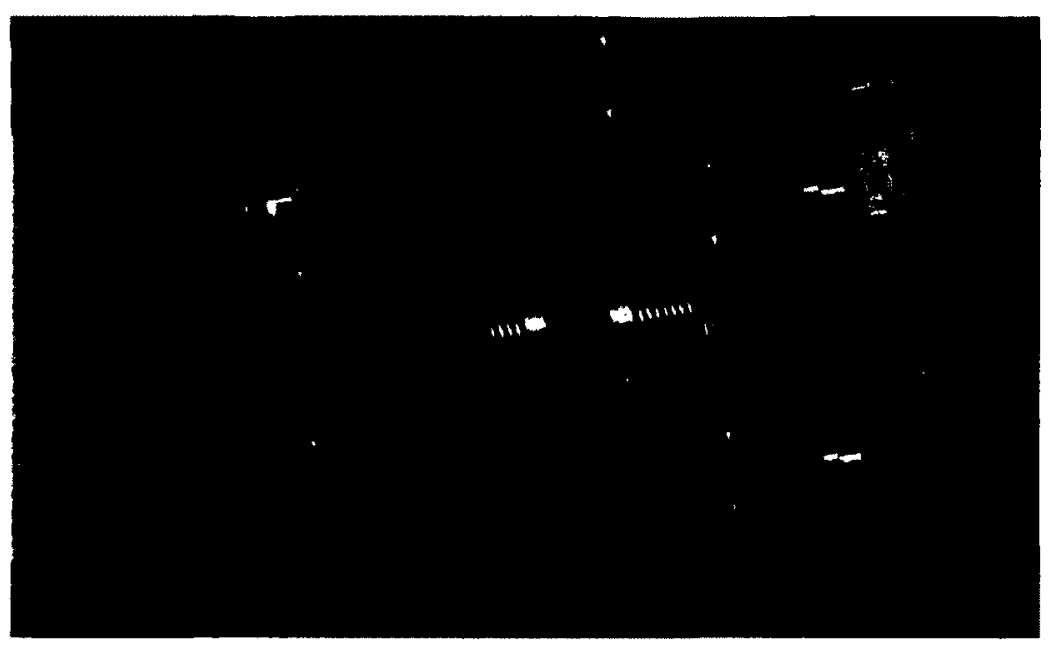

Figure 2.19: Exploded view of the end-effector illustrating the locking cone mechanism.

\subsubsection{Manufacture}

The entire foot plate was manufactured from aluminum due to ease of machinability, strength, and weight compared to steel. Figures 2.20(a) and 2.20(b) show the finished product with the magnet engaged and disengaged respectively. A proximity sensor was added on one side of the foot plate which informs the controller whether it is attached or not. The locking cones were originally chosen as steel, however they were changed to a hard plastic after initial tests damaged the surface. 


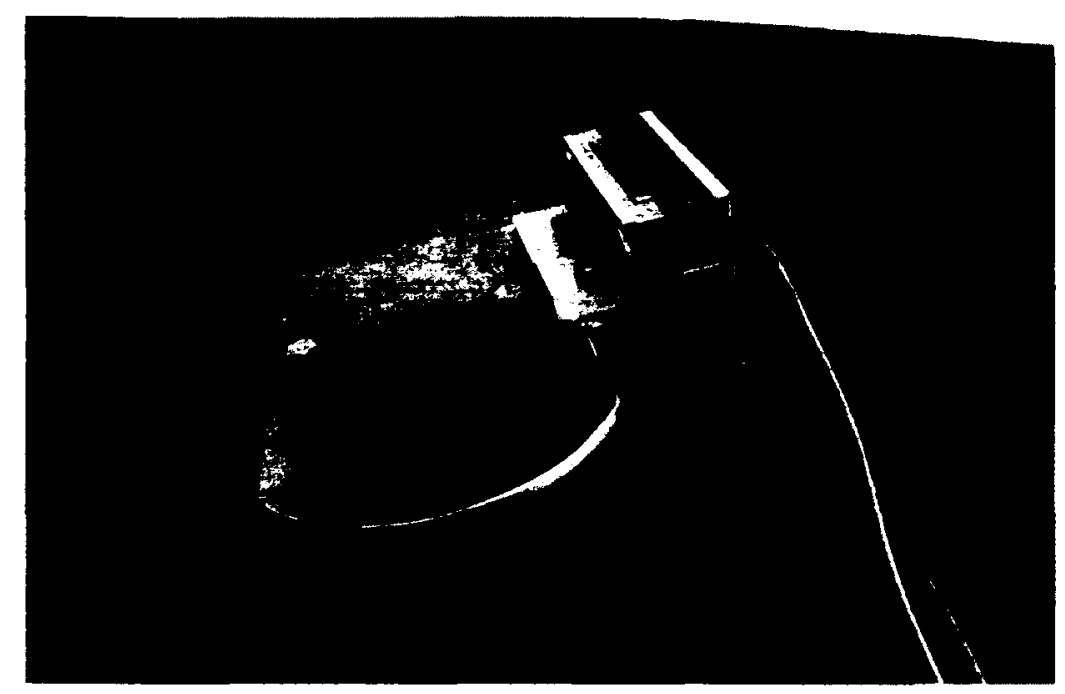

(a)

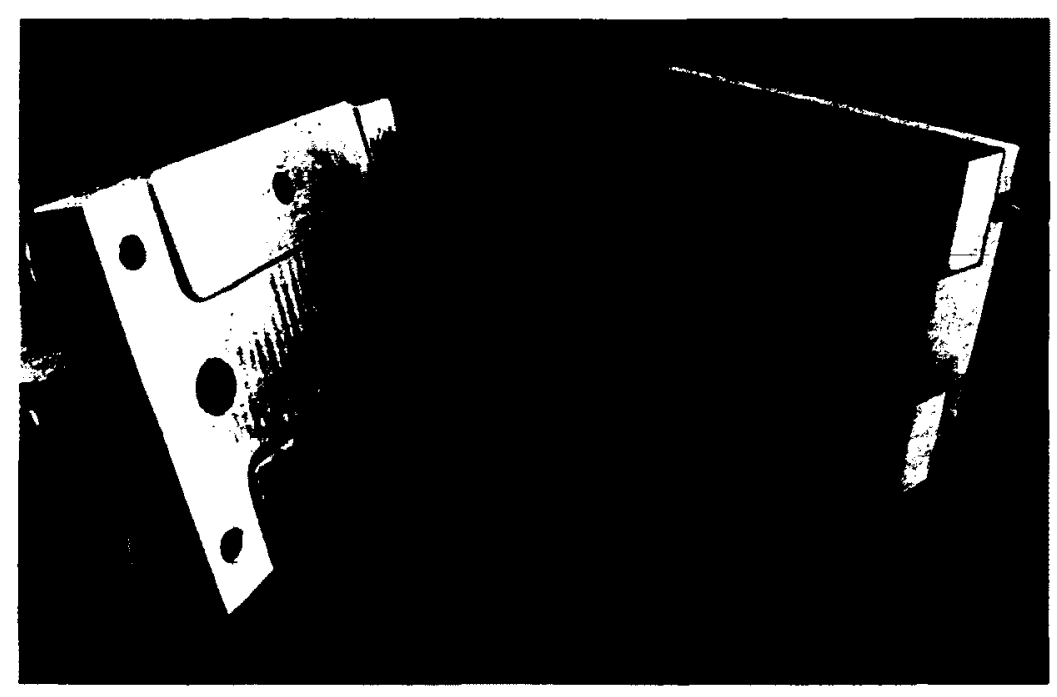

(b)

Figure 2.20: Manufactured end-effector with magnet engaged (a) and disengaged (b).

\subsubsection{Verification}

By knowing the force at which the foot plate will detach, a controller can be designed that varies the holding force on the magnets depending on the exercise being performed. Several experiments were conducted in order to determine the relationship between the forces required to detach the foot plate and the current flowing through the magnets. Neglecting 
a stop command from the controller, the foot plate can detach in six possible scenarios, a force in either $X, Y$, or $Z$ directions, or a moment about the $X, Y$, or $Z$ axis. After several trials, it was determined that the most likely separating conditions occurred when the foot plate is loaded with moments in the $X, Y$, and $Z$ axes as shown in Figure 2.21 below.

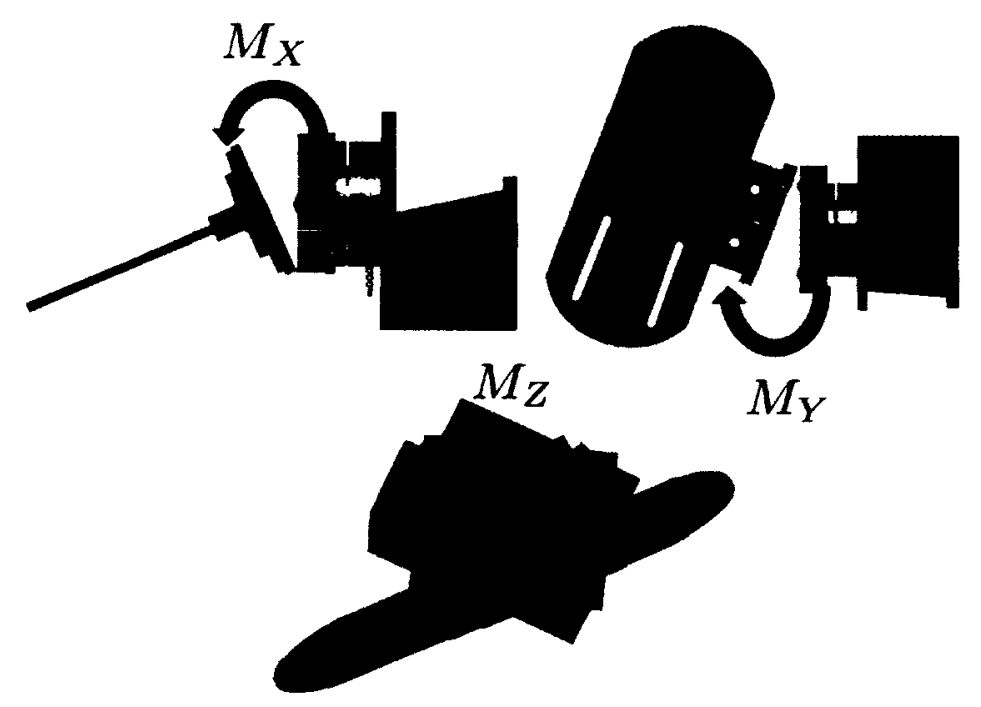

Figure 2.21: The 3 main decoupling conditions.

For each of the 3 different loading conditions, tests were conducted to determine the maximum break off moment for the amount of current supplied. Once a supply current was sent to an electromagnet, a moment would be applied to the foot plate. The moment at which the foot plate detaches was recorded for all 3 conditions. The results of these experiments can be seen in Figures 2.22, 2.23, and 2.24 below. These tests were conducted with only one magnet active so that an accurate representation of the holding force from a single magnet can be determined. Due to the symmetrical design of the foot plate, superposition can be used to determine the holding force of both magnets. In order to prevent damage to the foot plate or FT sensor, the maximum current supplied to the electromagnet did not exceed $45 \mathrm{~mA}$. Multiple break off moments were recorded for each current setting and are plotted as $x$ 's on Figures 2.22, 2.23, and 2.24. Large fluctuations in the break off data are attributed to the mating tolerance of the surface of the magnets and 
the steel plates. Small gaps in the mating surfaces cause large decreases in holding force.

Figure 2.22 shows the break off moment about the $X$ axis with only the bottom magnet active. There is an asymmetric loading pattern between $+M_{x}$ and $-M_{x}$. This is to be expected since the holding force of the electromagnet employs a small moment arm for $-M_{x}$ and a larger moment arm for $+M_{x}$. All other tests reveal symmetric loading patterns as to be expected.

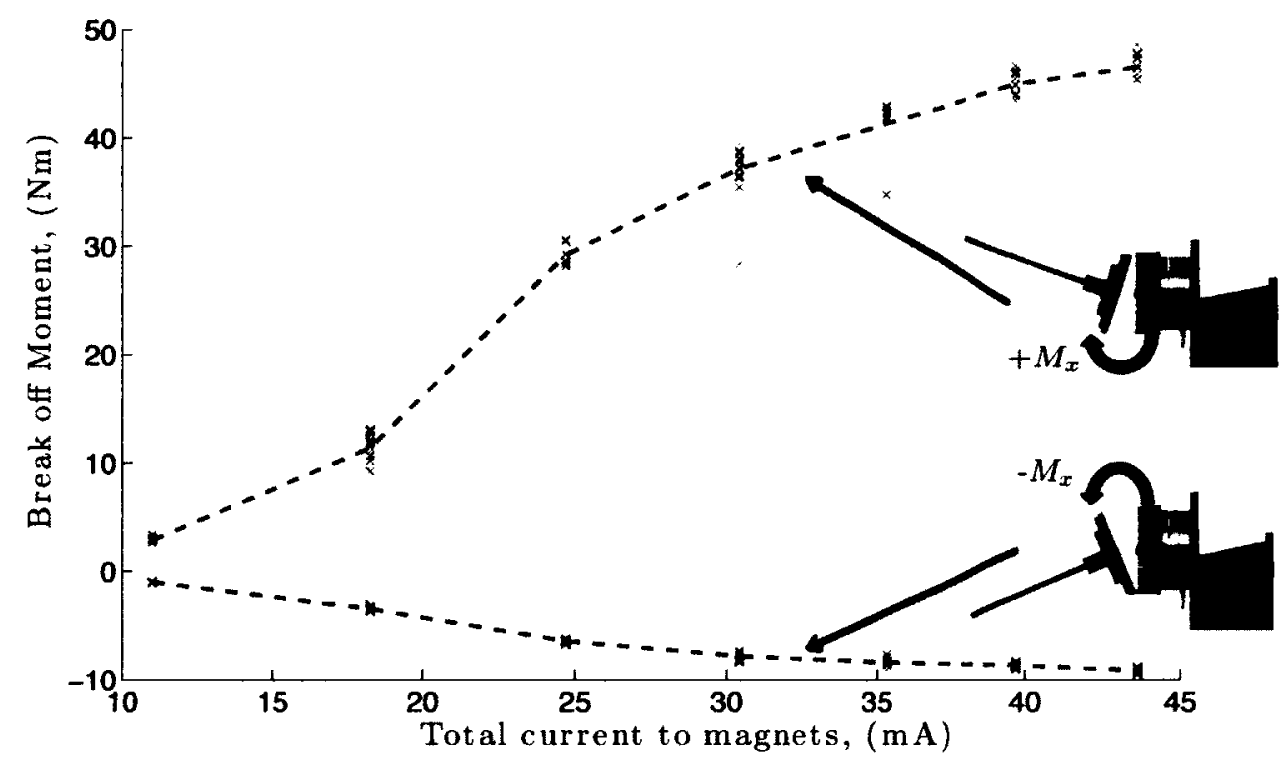

Figure 2.22: Break off moment about the $X$ axis for the bottom magnet. 


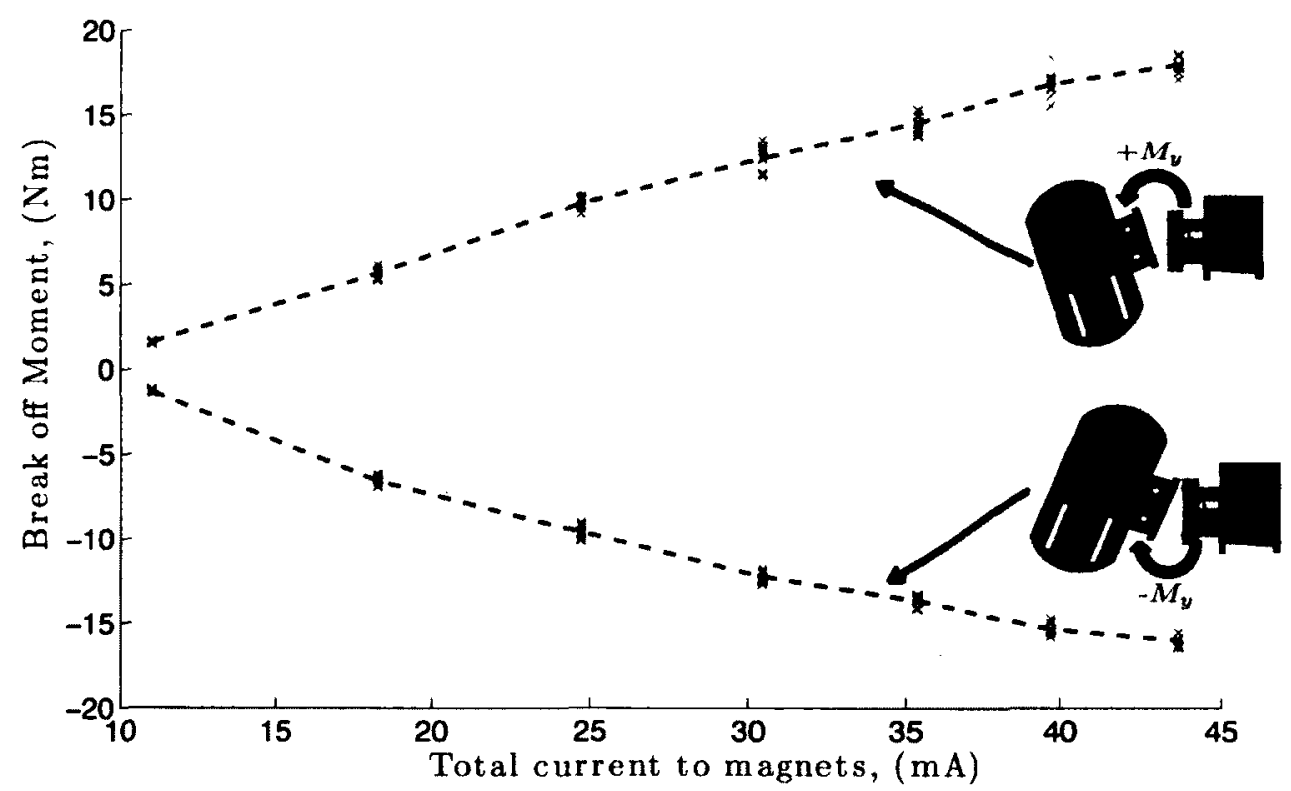

Figure 2.23: Break off moment about the $Y$ axis for the bottom magnet.

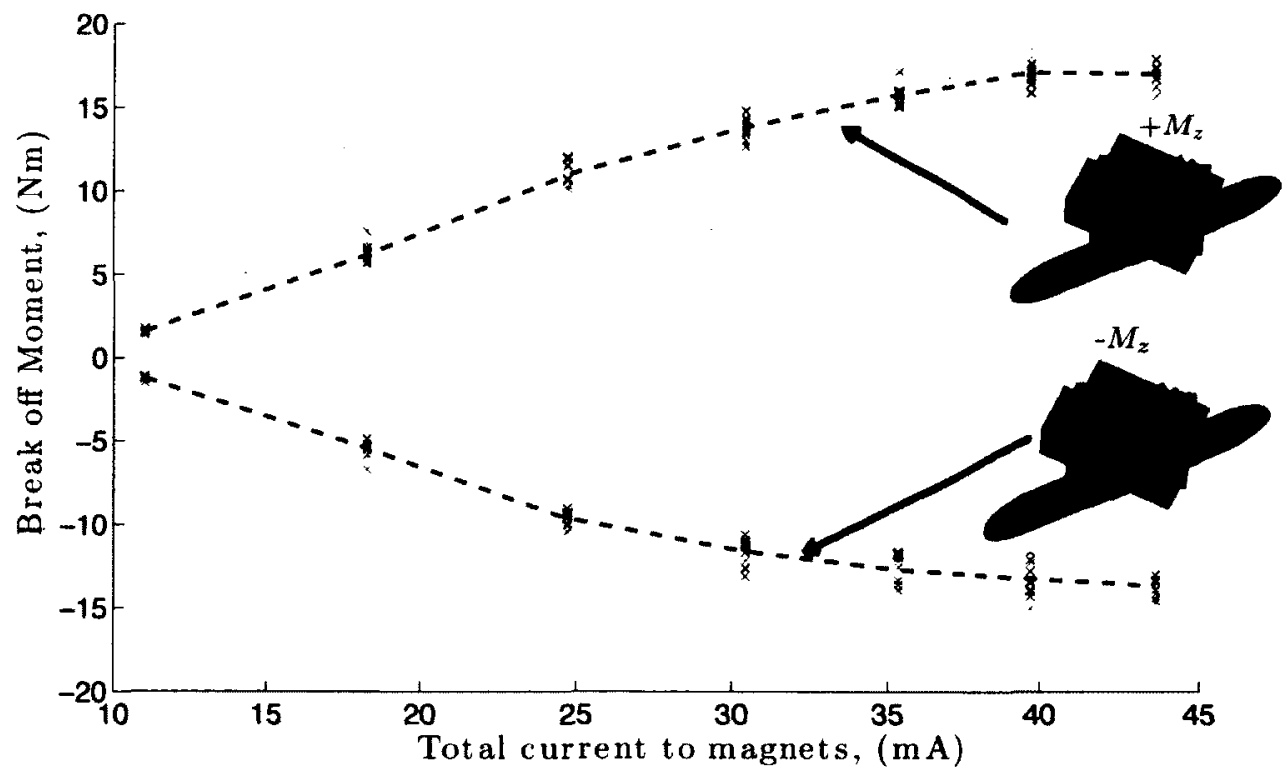

Figure 2.24: Break off moment about the $Z$ axis for the bottom magnet. 


\subsubsection{Discussion}

As can be seen in the previous sections, the foot plate was designed to comply with the requirements necessary for safe human-robot interaction. The foot plate was then verified to show that it is capable of detachment in a variety of loading conditions with a varying holding force. One requirement which was neglected in the modified foot plate design is the ability for the foot plate to provide metatarsel phalangeal flexion (flexion of the toe joints). This was purposefully neglected in order to simplify the design of the foot plate, however in future iterations this requirement should be included as it is a degree of freedom which is necessary for accurate reproduction of a normal gait cycle.

As stated above, the foot plate was fully designed out of aluminum due to ease of machining, and light weight compared to steel. Although the healthy subjects are capable of lifting the foot plate with the strength of their leg, this added weight provides a challenge for severely impaired patients. It is recommended that a material such as ABS plastic be used in the next iteration in order to reduce the weight associated with the foot plate.

\subsection{Summary}

This chapter focused on the software and hardware modifications necessary for ViGRR to function safely as a lower limb haptic rehabilitation device. A brief overview of ViGRR 1.0 was presented, along with the necessary design enhancements required for the ViGRR 1.1 upgrade. These design enhancements included a robot base platform, patient seat, interface board, limit switches, and updated kinematic calibration. The final design enhancement focused on the design, manufacture, and verification of a new foot plate for human-robot interaction. 


\section{Chapter 3}

\section{Haptic Delivery with ViGRR}

The haptic capabilities of the ViGRR platform, along with the development of a haptic rendering algorithm that is capable of displaying both forces and moments are described in detail in this chapter. This chapter will describe the approach taken to develop ViGRR as a haptic device with measures which can be compared to other similar devices.

The first section in this chapter briefly describes the high level force control scheme which sets the framework for the haptic rendering algorithm. Next, the theory and approaches to haptic rendering are discussed in order to provide a brief background. The haptic requirements of the ViGRR platform are then discussed and a haptic rendering algorithm is chosen based on those requirements. The algorithm was then implemented, and experiments were conducted which detail the performance of the algorithm with respect to the requirements. Once the haptic rendering has been implemented and verified, the generic performance measures of ViGRR as a haptic device can be defined.

\subsection{Admittance Control vs. Impedance Control}

There are two main approaches to force control of a haptic device: impedance control and admittance control. Both impedance control and admittance control can generate a dynamic behavior of a robot end-effector by setting a desired mass, damping, and stiffness. Although both control schemes can generate a desired dynamic behavior, the difference is in how the control schemes are implemented. Take for example, a robotic manipulator 
interacting with the environment under an inverse dynamics control in task space.

In its most primitive form, an impedance controller takes a trajectory as an input and ouputs the desired behavior of the robot directly to the inverse dynamics controller. This can be seen in Figure 3.1, where $\alpha$ is the control input to the inverse dynamics controller, $\tau$ is the computed torques sent to the motors, $F_{m}$ and $F_{d}$ represent the measured and desired forces respectively, $\dot{x}_{d}$ and $x_{d}$ represent the desired velocity and position of the endeffector, and $\dot{x}_{m}$ and $x_{m}$ represent the measured velocity and position of the end-effector. In this control scheme, the impedance control and motion control are computed in the same step, coupling the control objectives. However, it is often required to have separate control objectives for free space where the control objective is to track motion with low error, and interaction where the control objective is to provide a desired dynamic behavior with the end-effector.

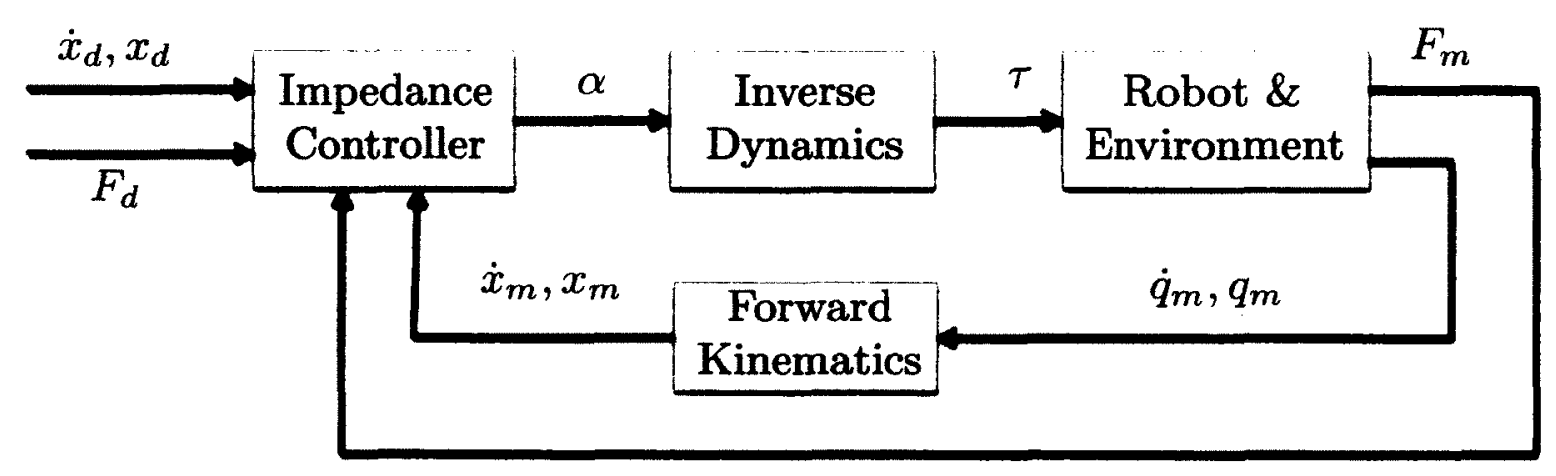

Figure 3.1: Block Diagram of the impedance control scheme.

Admittance control attempts to solve this issue by decoupling the impedance control from the motion control. This allows the motion control to be purposefully stiff, whereas the impedance control parameters can be chosen at will. Essentially, the admittance controller acts like a trajectory generator, taking a force input and producing a desired position $x_{d}$ and velocity $\dot{x}_{d}$ in task space. A separate, stiffer motion controller can then be used to generate an input to the inverse dynamics control law as seen in Figure 3.2. An obvious benefit to the admittance control scheme is the range of end-effector impedances that can be delivered without reduction in motion control performance. 


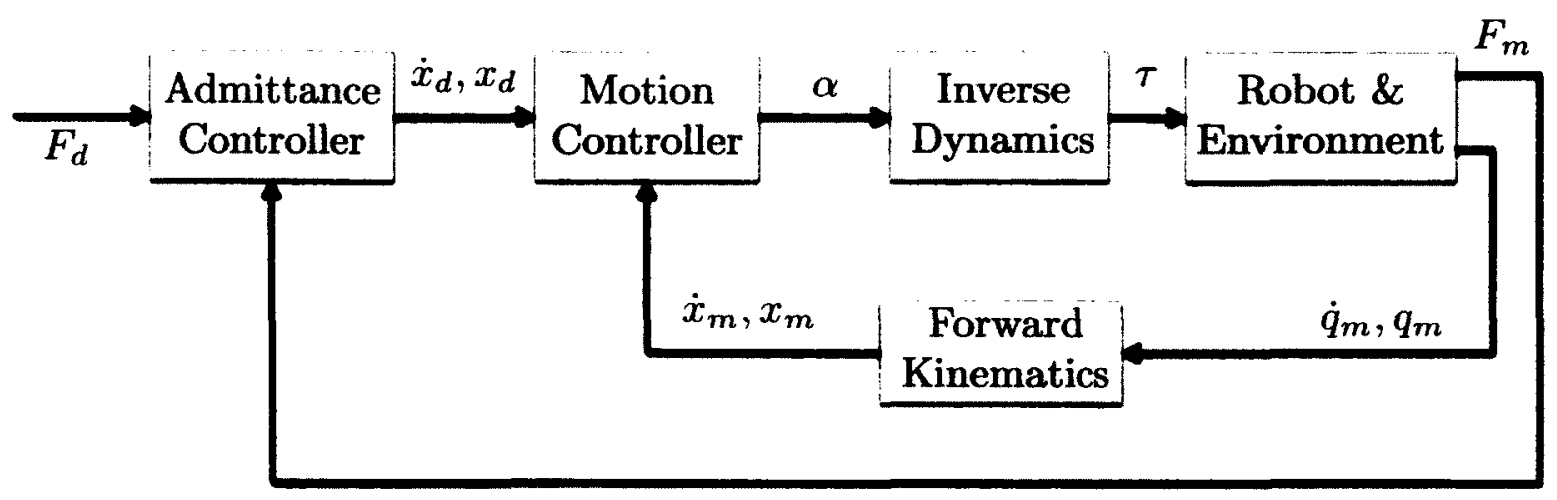

Figure 3.2: Block Diagram of the admittance control scheme.

\subsubsection{ViGRR Admittance Control}

ViGRR is designed to produce a variety of behaviors depending on the necessity of the user. The wide range of dynamic behaviors required from the end-effector motivated the development of an admittance control scheme. As stated above, admittance control produces a desired trajectory in task space from a force measured at the end-effector. If a desired force is requested, such as in the case of a virtual wall contact, the robot will attempt to match the measured force to the desired force. More formally, the desired trajectory of the ViGRR end-effector can be written as

$$
\ddot{x}_{h}=\frac{1}{m_{h}}\left(F_{m}-F_{d}-B \dot{x}_{h}\right),
$$

where $m_{h}$ and $B$ specify the desired mass and damping respectively. $F_{m}$ represents the measured force from the FT sensor and $F_{d}$ represents the desired forces generated from the haptic rendering. Most often, it is necessary to add a dead zone to $F_{m}$ which prevents the sensor noise from sending commands to the admittance control. For ViGRR, this dead zone prevents forces less than $1 \mathrm{~N}$ and torques less than $0.1 \mathrm{Nm}$ from generating task space trajectories. These deadzones are compatible with the noise levels of the ATI force sensor at hand.

The desired acceleration $\ddot{x}_{h}$ is integrated twice to calculate the desired position of the end-effector. This end-effector position $x_{h}$ is then fed through a redundancy resolution scheme and inverse kinematics to determine the desired position $q_{d}$ and velocity $\dot{q}_{d}$ of each 
joint. The joint angles and velocities are then used to calculate the desired torque required for each motor through

$$
\tau=P e+D \dot{e}+G\left(q_{m}\right)
$$

where

$$
\begin{aligned}
& e=q_{d}-q_{m}, \\
& \dot{e}=\dot{q}_{d}-\dot{q}_{m},
\end{aligned}
$$

where $P$ and $D$ are constant gains and $G$ represents the gravity compensation term which is a function of the robot's measured position $q_{m}$. Although the current control scheme is capable of relatively small tracking errors $\left(<0.05^{\circ}\right)$, it is recommended to include a dynamic model of the robot in future work to allow for uniform performance at any configuration. Figure 3.3 shows a block diagram of the control strategy used during the walking experiments. The blocks in green represent the controller, whereas the blue, red, and purple represent the robot, sensors, and haptic rendering algorithm respectively. 


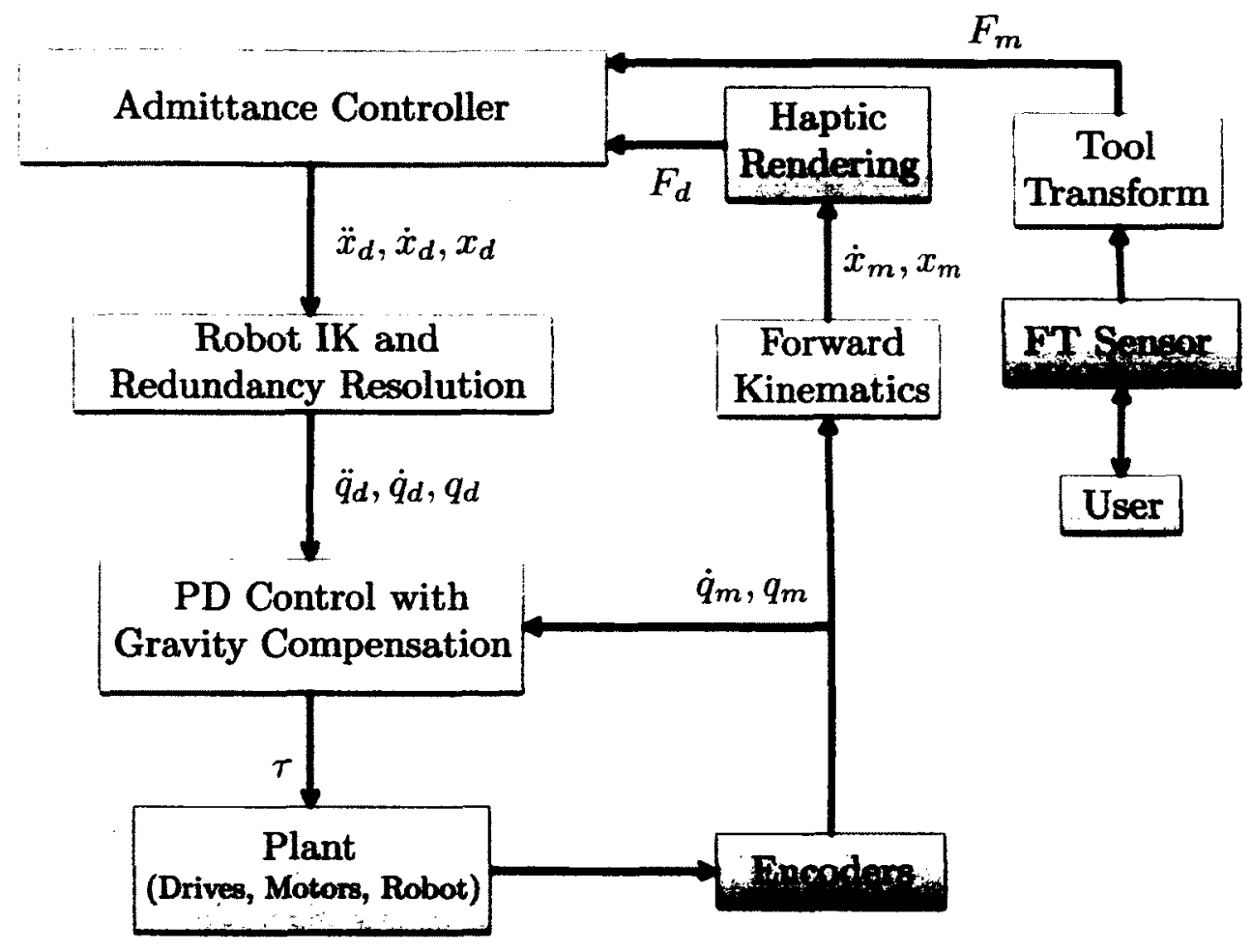

Figure 3.3: Block Diagram of the ViGRR control scheme.

\subsection{Haptics Theory}

When we touch objects in real life, we sense forces based on the contact between our body and the environment. These forces give us a sense of the properties of our environment such as the location of objects, mass, texture, etc. Haptic rendering is the process of transforming forces from a virtual environment to the real world through an external interface such as a robot. This process creates the illusion of touching a virtual object.

Haptic rendering is composed of two parts, a physical system and a virtual world. The physical system is comprised of a haptic device and user, whereas the virtual world consists of a virtual tool and the environment that it interacts with. This framework is akin to master-slave teleoperation. There are two main problems associated with haptic rendering: finding the configuration of the virtual tool and displaying the forces associated with the configuration of the tool. One formal definition of this problem is written as: 
Given a configuration of the haptic device $H$, find a configuration of the tool $T$ that minimizes an objective function $f(H, T)$, subject to environment constraints. Display to the user a force $F(H, T)$ dependent on the configurations of the device and the tool [18].

This problem can be formulated into two computational steps: collision detection and force computation.

\subsubsection{Collision Detection}

Collision detection is the computational step which determines if the virtual tool is in contact

with the virtual environment. Usually this is the step which requires the most computing time if there are many objects in the environment or if the objects are geometrically complex.

Polygonal models are common in haptic rendering, however they can quickly become computationally expensive if the objects are complex. Most haptic rendering algorithms that use polygonal models will also use a form of spatial partitioning or bounding volume hierarchies (BVHs) in order to speed up the algorithm. Spatial partitioning is a recursive partitioning of the embedding space, whereas bounding volume hierarchies are based on a recursive partitioning of the primitives of an object. Some examples of spatial partitioning include k-D trees, octrees, R-trees, cone trees and BSPs. Some examples of BVHs include sphere-trees, AABB-trees, OBB-trees, spherical shell-trees, and k-DOP trees [18].

Volume-based collision detection is of particular interest to haptic rendering techniques as the computation time is independent of the number of polygons. The most common volume-based collision detection method is the Voxmap-PointShell ${ }^{T M}$ (VPS) Algorithm developed by McNeely [50]. This method divides the virtual scene into a dynamic and static object. The static object is then partitioned into a set of volume pixels (voxels) of uniform size. The dynamic object is transformed into a pointshell which is essentially a cloud of points that are located along the outer surface of the object. A collision has occurred if a point on the pointshell is located within a voxel of the static object.

The above methods are most useful with polygonal models, however collision detection 
techniques are constantly being developed for use with non-convex models, non-uniform rational b-splines (NURBS), and deformable models [18].

\subsubsection{Force Computation}

Force computation is the computational step which determines the forces that are applied to the haptic device due to the configuration of the tool and the environment. The forces can be rendered in either 3-DoF or 6-DoF depending on the application. 3-DoF rendering interprets the virtual tool to be represented as a point, greatly reducing the computation time and complexity. 6-DoF rendering produces a six dimensional force/torque vector to be applied to the haptic device. As with collision detection, there are several approaches to computing the forces for haptic rendering: Penalty-based, Impulse-based, and Constraintbased.

Penalty based force algorithms are the most common method used for haptic rendering. These approaches compute interaction forces based on the intersection depth of two colliding bodies. Penalty based methods are inherently poor at representing hard objects. Impulse based algorithms generate an impulse each time a collision occurs which gives the effect of a hard contact. If poorly designed, impulse based approaches can lead to instability by adding energy to the system. Penalty based methods are most commonly used in combination with impulse based algorithms to offset each other's limitations [50].

Constraint based approaches involve geometrically constraining the virtual tool to the environment if there is a collision. The forces are then computed based on the contact geometry by solving the linear complimentary problem (LCP) which is common in many rigid body dynamics simulations. One of the first constraint based approaches was introduced by Zilles and Salisbury which made use of a 3-DoF "god-object" [51].

\subsubsection{Direct Rendering vs. Virtual Coupling}

There are two approaches which can be used to generate the forces on a haptic device depending on the virtual tool configuration. 
The first is direct rendering which determines the position and orientation of the virtual tool by directly relating it to the position and orientation of the haptic device. The contact force is then fed directly into the controller for the haptic device. Figure 3.4 shows the block diagram of the process. This method is attractive in its simplicity to implement since there is no need for a complex rigid body optimization. A big disadvantage of this method arises from instability if the update rate drops low enough to allow for large penetration depths.

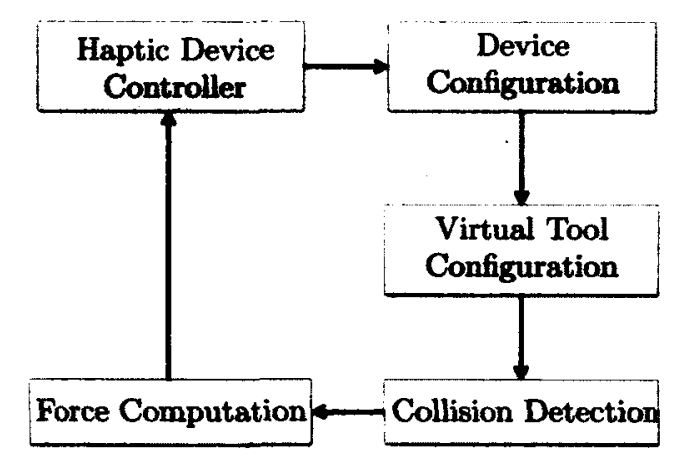

Figure 3.4: Block diagram of direct rendering.

Virtual coupling is the second rendering approach which attaches a passive coupling network, such as a spring and damper between the haptic device and virtual tool. In this fashion, the addition of passive devices leads to a more stable system [18]. Haptic rendering through virtual coupling introduces complex dynamics to the system due to the added compliance. The block diagram of the virtual coupling method can be seen in Fig 3.5. With virtual coupling, the force that is generated after collision detection gets fed through a tool solver. The force that is output to the controller is determined by the difference in configurations of the virtual tool and haptic device. If the configurations of both are equal, no force is output to the controller.

An example of virtual coupling can be seen in Fig 3.6, where the wire frame spoon represents the position of the haptic device and the solid spoon represents the position of the virtual tool. Clearly the haptic device is in an impossible configuration as it is passing through the cup. The solid spoon represents the best solution as determined by the tool solver and a force is then generated due to the misalignment of the two objects and fed to the controller. 


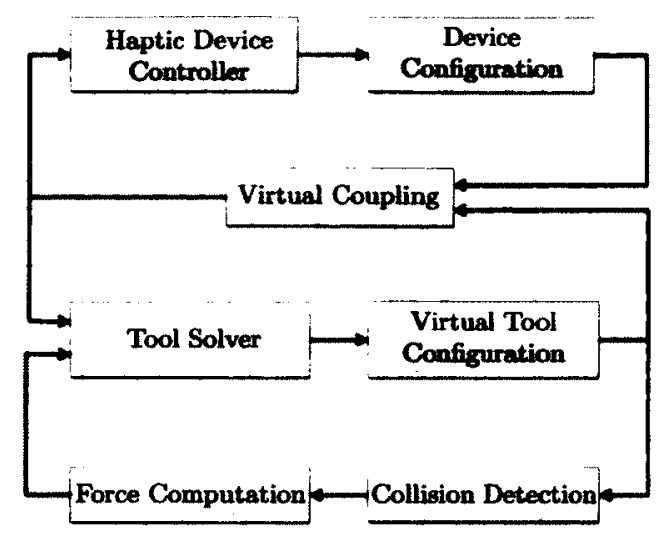

Figure 3.5: Block diagram of virtual coupling.

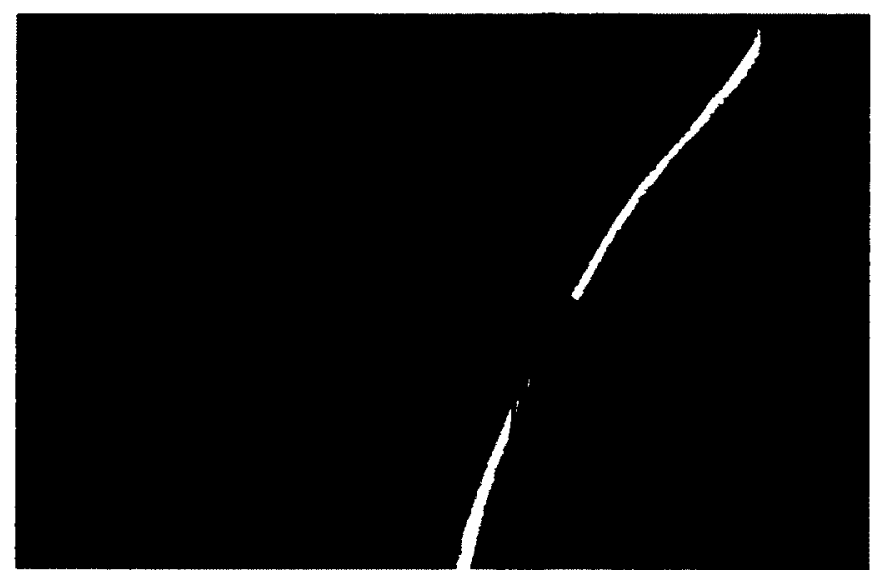

Figure 3.6: Example of virtual coupling. The wire frame spoon is the position and orientation of the haptic device and the solid spoon is the closest possible solution [18].

\subsection{Haptic Rendering Requirements}

Haptic devices are designed in order to couple the user to a virtual environment by providing a sense of touch within that environment. As discussed in Chapter 2, the ViGRR platform is designed to function as a lower limb haptic robot. More specifically, ViGRR is designed to provide the sense of walking to the user. Unlike most haptic devices that are designed for the hand, ViGRR has a very unique set of haptic requirements that are needed to impart these walking forces. 


\section{Degrees of Freedom}

In order to impart ground reaction forces to the user's foot, the ViGRR haptic rendering algorithm must be able to generate forces in the $X$ and $Y$ directions and a moment about the $Z$ axis. Without a moment about the $Z$ axis, the user would feel as if their foot is pivoting about a single point attached to their foot. Although ViGRR 1.1 is constrained to the $X Y$ plane, future iterations will incorporate more degrees of freedom. For these reasons, a 6-DoF haptic rendering technique is required.

\section{Friction}

Forces due to friction are essential components of ground reaction forces that are felt during walking and contribute directly to the user's stability. If neglected from the rendering algorithm, the user will feel as if they are walking on a slippery surface. For this reason, friction must be included in the haptic rendering.

\section{Variable Contact Parameters}

ViGRR is designed to handle a wide variety of users ranging from healthy to severely impaired. The capabilities of these users to generate forces and moments on virtual objects also varies significantly. A very stiff contact with the environment would be necessary for a healthy individual. For a severely impaired user, it might be more appropriate to model all environment contacts with a low stiffness to prevent harm. The contact parameters of the haptic rendering algorithm must therefore be adjustable.

\section{Real-Time Compatibility}

ViGRR is designed to operate on a QNX real-time computer at a minimum loop rate of $1 \mathrm{kHz}$. A widely accepted loop rate for immersive haptic simulations also requires a minimum of $1 \mathrm{kHz}$ [50]. Therefore, any haptic rendering algorithm which would be implemented on ViGRR must also be compatible with QNX, running at a minimum of $1 \mathrm{kHz}$. 


\subsection{Haptic Rendering Algorithm}

Haptic rendering is the process of generating forces and torques based on the position of the haptic device. Extensive research has gone into developing techniques that are computationally quick, stable, and accurate in the forces that are provided. Many haptic APIs have been developed which are available as open source software (CHAI3D, JTouchTool, libNiFalcon, HAPI, H3D API). Some of these APIs, however, are not applicable to our application due to the inherent drawbacks of the algorithm (eg. CHAI3D only provides 3-DoF rendering), and compatibility issues with our software. For these reasons we have chosen to develop our own 6-DoF haptic rendering algorithm.

This strategy involves the integration of the rigid body simulator Open Dynamics Engine (ODE) [19]. ODE is an open source software which allows the dynamic simulation of rigid bodies in a virtual world. The software includes built in collision detection, force computation, and friction models which are used in the haptic rendering process. Another benefit of using ODE for the haptic rendering algorithm is that the software is used primarily as a rigid body simulator which is useful for interacting.with dynamic objects. Rehabilitation excercises and games are simple to implement for ViGRR with the use of the ODE software.

\subsubsection{Open Dynamics Engine}

Open Dynamics Engine is a free and open source rigid body dynamics simulation tool. Since the focus of this paper is on the application of a haptic algorithm and not on the development of a novel haptic rendering technique, certain aspects of the controller will involve the ODE simulation. Although ODE is open source and is described in detail online, relevant information will be described as it is important to the function of the haptic rendering algorithm.

\section{Collision Detection}

Simple primitives such as boxes, cones and spheres can be created using ODE's built-in functions. More complex geometry (triangle "soup") can be imported into the simulation by 
creating a triangle mesh polygon. Every polygon in ODE gets partitioned into axis aligned bounding boxes (AABBs) in order to speed up the computation time. When two objects have intersected, the simulation calls a user defined function where certain parameters of the collision (friction, coefficient of restitution, etc.) can be set.

\section{Contact Force Model}

Open Dynamics Engine uses a constraint based approach to generate the contact forces acting on objects in the simulation. This approach gives the effect of hard contacts and more realistic simulations as opposed to penalty-based methods. For a single rigid body, the Newton Euler equation of motion can be written as

$$
F=M \dot{v}-F_{\text {ext }},
$$

where $F \in \Re^{6}$ is the sum of all forces acting on the body, $F_{\text {ext }} \in \Re^{6}$ is the sum of external forces acting on the rigid body, $M \in \Re^{6 \times 6}$ is the mass-inertia matrix, and $\dot{v} \in \Re^{6}$ is the acceleration of the body's centre of mass. For unconstrained motion, (3.4) holds at any given instant. If, however the rigid body is fixed at a joint or contacting other geometry, an extra term must be added in order to satisfy the constraint. The constraint equation is formulated for every joint as

$$
J v=0
$$

and every contact as

$$
J v \geq 0
$$

The matrix $J \in \Re^{6 \times 6}$ is a function of the position of the rigid body and is used to relate the body's velocity to a constraint. The matrix $J$ is called the Jacobian and can be expanded to incorporate multiple bodies with multiple constraints. At every timestep, a vector $\lambda$ is 
calculated such that the force applied to the rigid body to preserve the constraint is

$$
F_{c}=J^{T} \lambda
$$

The vector $\lambda \in \Re^{6}$ is called the coefficient vector and contains the magnitudes of the constraint forces. For a rigid body with constraints, (3.4) can be rewritten as

$$
F=J^{T} \lambda+F_{e x t}=M \dot{v}
$$

From above, $\lambda$ can be calculated by reformulating (3.8) and the constraint equations (3.5) and (3.6) into a linear complimentary problem (LCP) which is common in many rigid body dynamics simulations. ODE uses a method based on Dantzig's algorithm to solve this problem which is described in detail in [52].

\section{The Error Reduction Parameter and Constraint Force Mixing}

ODE introduces two parameters which can be independantly set in order to maintain the stability of a contact or joint. The first is the Error Reduction Parameter (ERP) which attempts to correct for errors that can cause the bodies to drift from their desired positions such as in a rigid joint. During each simulation step, the joint applies a correcting force which is controlled by the ERP (set from 0 to 1 ). The second parameter is called Constraint Force Mixing (CFM) and is used to allow the constraint to be violated by an amount proportional to CFM times the restoring force that is needed to enforce the constraint. CFM is added to the constraint equation as

$$
J v \geq C F M \lambda \text {. }
$$

Adjusting both CFM and ERP have the effect of adding a spring and damper to the contacts and joints in the simulation. Mathematically, a desired spring constant $k_{p}$ and damping constant $k_{d}$ for any contact can be found through

$$
E R P=\frac{h k_{p}}{h k_{p}+k_{d}}
$$


and

$$
C F M=\frac{1}{h k_{p}+k_{d}}
$$

where $h$ is the timestep of the simulation. For all experiments in this thesis, the ERP and CFM were set to 0.96 and 0.04 respectively.

\section{Friction Model}

Traditionally, the LCP solution is applicable to frictionless rigid bodies only. ODE adds friction to the simulation by approximating the coulomb friction model

$$
\left|F_{T}\right| \leq \mu\left|F_{N}\right|
$$

where $\mu$ is the coefficient of friction (a number between 0 and 1), and $F_{T}$ and $F_{N}$ are the tangential and normal force vectors respectively. This equation defines a cone as seen in Fig 3.7 where the axis of the cone is the normal vector and the vertex is the contact point. If $\vec{R}$ lies inside the cone, the friction force is large enough to hold the object in place whereas if it lies on or outside the cone, the object will move.

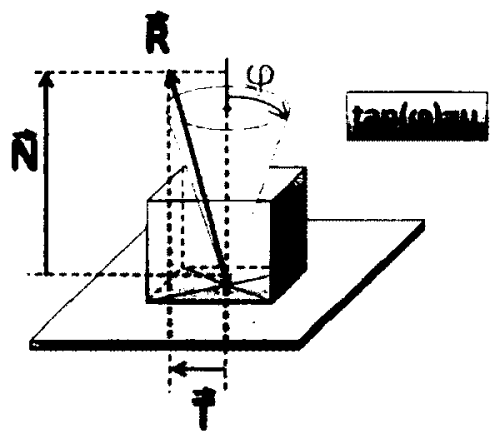

Figure 3.7: Graphical representation of the friction cone. $\vec{N}$ is the normal force, $\vec{T}$ is the tangential force and $\vec{R}$ is the resultant of the two [19].

For efficiency reasons, the coulomb friction model is approximated during simulation using two different methods. The first, and also the most computationally efficient approximation, is to define a force threshold that is independent of the normal force. If the 
tangential force becomes larger than this threshold, then the object will move. The second friction approximation simplifies the friction cone as a pyramid which is aligned with the direction of travel. ODE first computes the normal forces assuming frictionless surfaces. It then determines the maximum limits for the tangential forces based on those normal forces and solves the entire system with these limits [19]. This approximation is more accurate, although it is also more computationally expensive with large simulations. However, the real-time computer used for controlling ViGRR is capable of handling a large number of objects with the more accurate friction approximation.

\subsubsection{The Algorithm}

This section describes the haptic rendering algorithm developed for ViGRR. The algorithm is based on the virtual coupling technique to maintain a more stable rendering and allow for variable contact parameters. Throughout this paper, the term haptic device will be used to describe the end-effector of ViGRR and the term virtual tool will be used to describe the object defined within the ODE environment.

To start, a virtual world is created in ODE which contains all colliding bodies in the scene. The virtual tool is given geometric and mass properties which will determine how it interacts with its environment. Figure 3.8(a) shows the virtual world with a ground and a box representing both the haptic device and virtual tool. Only one box can be seen since the virtual tool and haptic device are coincident. The ViGRR robot is included for visual purposes and does not have a virtual body associated with it. The position of the haptic device $x_{h}$ is determined from the device controller whereas the position of the virtual tool $x_{v}$ is determined by a coupling force $F_{d}$. This coupling force attempts to keep the virtual object at the same position and orientation as the haptic device through the equation

$$
F_{d}=K\left(x_{h}-x_{v}\right)+D\left(\dot{x}_{h}-\dot{x}_{v}\right)
$$

where $K \in \Re^{3 \times 3}$ and $D \in \Re^{3 \times 3}$ represent the diagonal stiffness and damping matrices respectively, $x_{h} \in \Re^{3}$ and $\dot{x}_{h} \in \Re^{3}$ represent the position and velocity of the haptic device, 
and $x_{v} \in \Re^{3}$ and $\dot{x}_{v} \in \Re^{3}$ represent the position and velocity of the virtual tool. Therefore, if there is a large discrepancy in the positions of the virtual object and haptic device, the coupling force $F_{d}$ will be generated such that it will attempt to minimize that error. This can be seen in Figure 3.8(b) below where the virtual object is in contact with the ground.

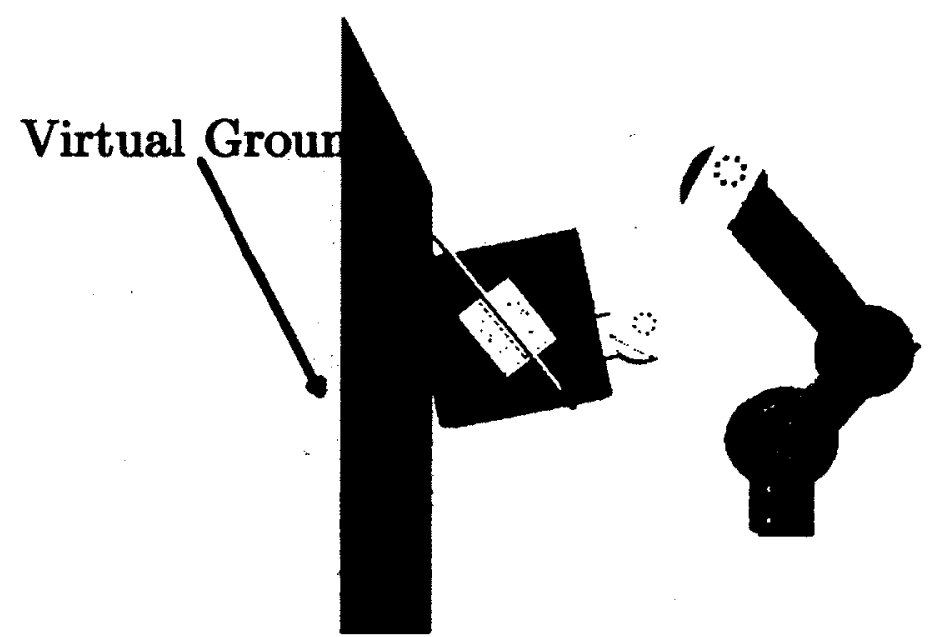

(a) Virtual tool in free space.

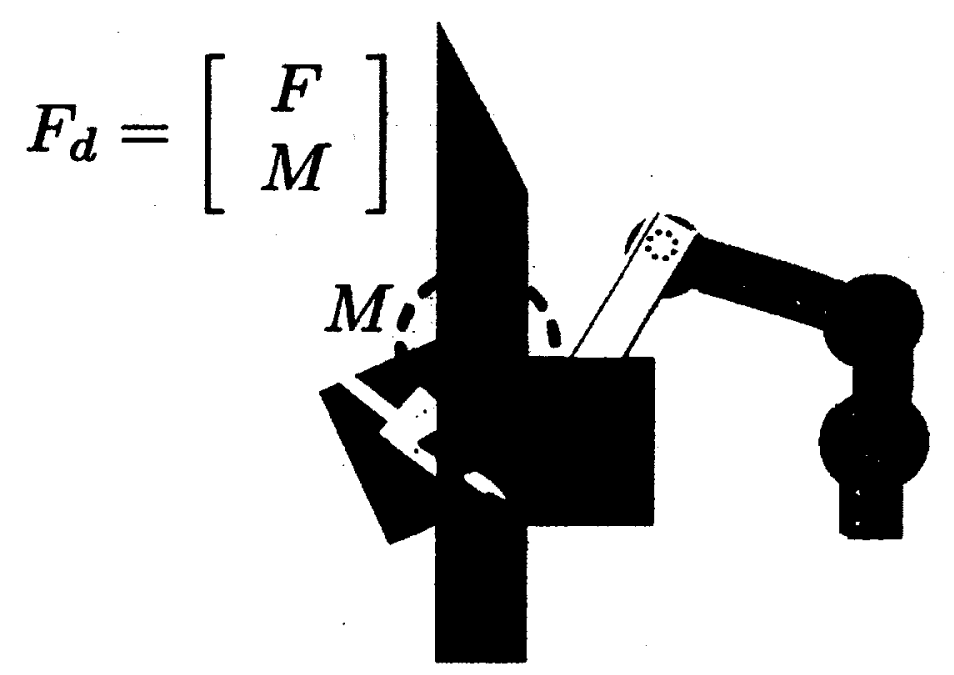

(b) Virtual tool in contact with wall.

Figure 3.8: Virtual world showing the haptic device (red box) and the virtual tool (blue box).

Figure 3.9 shows the block diagram of the haptic rendering algorithm. The blocks inside the dotted red box indicate the processes that are taken care of within ODE. 


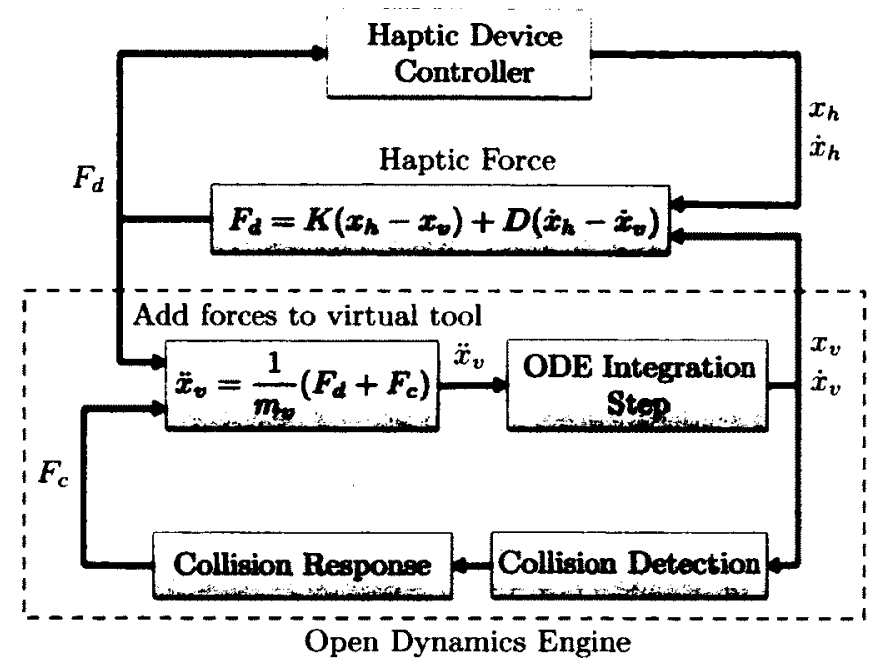

Figure 3.9: Block diagram of the haptic controller.

As can be seen, the coupling force $F_{d}$ determines the position of the virtual tool, it also acts as the desired force which is sent to the haptic device controller. Therefore, the position and velocity of the virtual tool directly affect the forces felt by the user. Caution must be taken in choosing $K$ and $D$ so that the virtual tool maintains stability. As shown in Figure 3.9, the acceleration of the virtual tool is governed by the equation

$$
\ddot{x}_{v}=\frac{1}{m_{v}}\left(F_{d}+F_{c}\right) .
$$

In free space, the contact force $F_{c}$ is zero and the dynamics of the virtual tool can be found by combining (3.14) and (3.13) to obtain

$$
\ddot{x}_{v}-\frac{D}{m_{v}}\left(\dot{x}_{h}-\dot{x}_{v}\right)-\frac{K}{m_{v}}\left(x_{h}-x_{v}\right)=0,
$$

where $m_{v}$ is the mass of the virtual tool. The damping ratio for this system is then defined as

$$
\zeta=\frac{D}{2 \sqrt{0.1 K}}
$$

For a critically damped system, $D$ can be chosen such that 


$$
D=2 \sqrt{0.1 K}
$$

\subsection{Haptic Rendering Implementation}

The controller implemented on ViGRR is compiled for the real-time QNX target using Simulink and Quarc. In order for the haptic rendering algorithm to be compatible with the existing code, the ODE library must first be compiled for QNX using the QNX Momentix IDE. The algorithm can then be placed in a Simulink $\mathrm{C}++$ S-Function block and compiled for real-time control. The controller for ViGRR is running at a consistent loop rate of $2 \mathrm{kHz}$, while simultaneously sending relevant visualization data through TCP/IP to the host computer.

The graphics for the haptic rendering algorithm utilizes the Quarc 3D visualization interface which generates 3D models using a proprietary X3D data format, $q x 3 d$. The 3D models for ViGRR are first exported from CAD software to stereolithography (.stl) format, then imported into an open source 3D graphics software, Blender. The stl files can then be exported from Blender into qx3d format. The host computer renders the visualization at $30 \mathrm{~Hz}$ to prevent a loss of visual fidelity. A graphical user interface (GUI) is also implemented on the host computer which allows the operator to change parameters and controllers while online. Figure 3.10 shows a block diagram of the parallel processes which are implemented on ViGRR. 


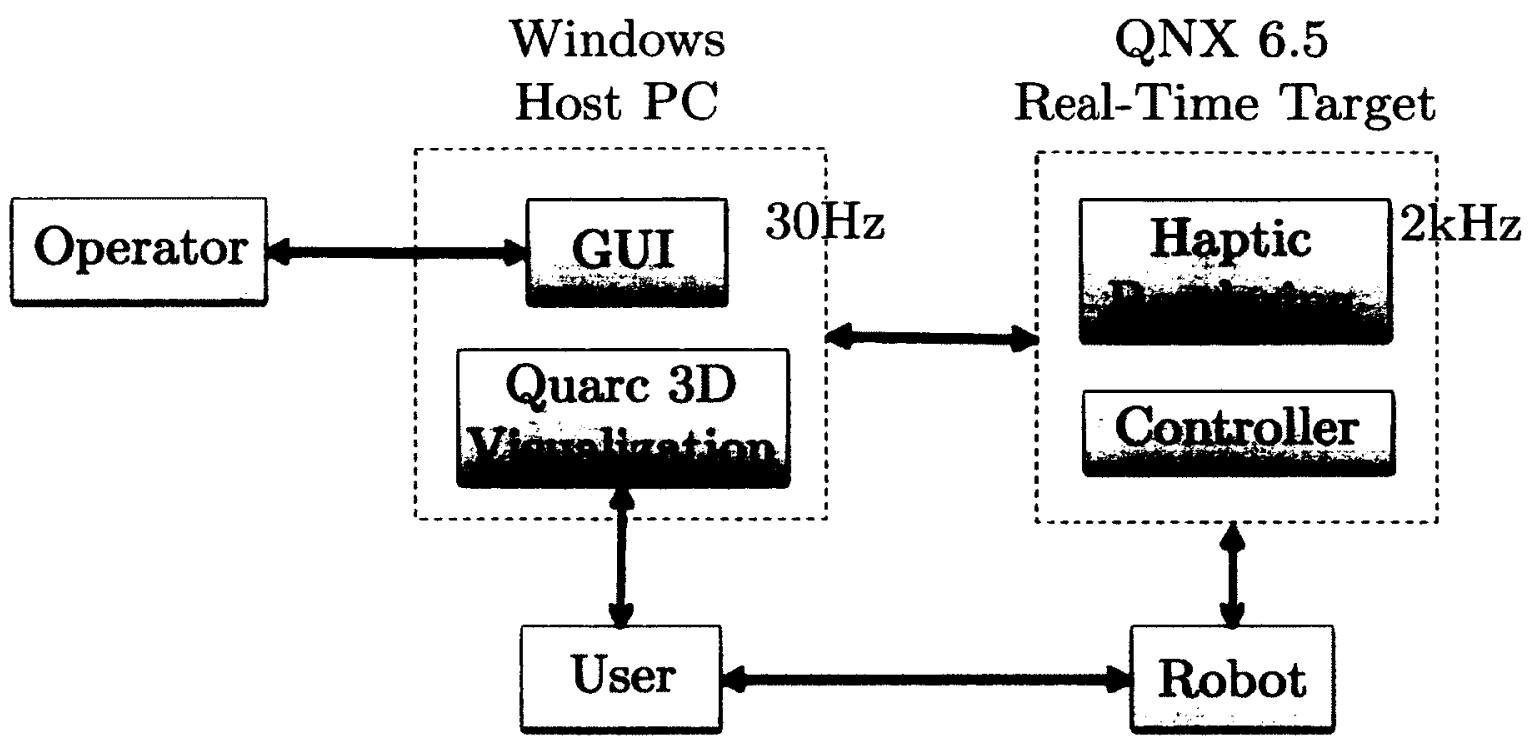

Figure 3.10: Block diagram of the parallel processes of ViGRR

\subsection{Haptic Rendering Verification}

In order to prove that the haptic rendering algorithm can provide realistic forces to the user, a test program was developed with a virtual wall located at $X=0.72$ meters from the ViGRR base. The foot plate was grasped by the user's hand, and contact forces were generated by moving the haptic device into the virtual wall.

\subsubsection{Effects of Varying $K$ and $D$}

The haptic rendering algorithm utilizes spring and damping parameters $K$ and $D$ which can be varied in order to achieve different desired static contact forces. In order to simplify the contact model, the virtual tool was chosen as a sphere which provides only one point of contact with the virtual wall and can be seen in Figure 3.11. Figure 3.12 shows the contact forces of two separate trials. The two lefthand graphs show a contact with the wall with a $K$ value of 1000 and a $D$ value of 10 . The middle graphs show a similar wall contact with $K=4000$ and $D=10$. The righthand graphs show the resultant position and force output with the damping increased to 100 . The dotted line on the top graphs show the position 
of the virtual tool and the solid line shows the position of the haptic device. Likewise, the dotted line on the bottom graphs show the contact forces produced by the virtual tool and the solid line shows the measured forces from the FT sensor.

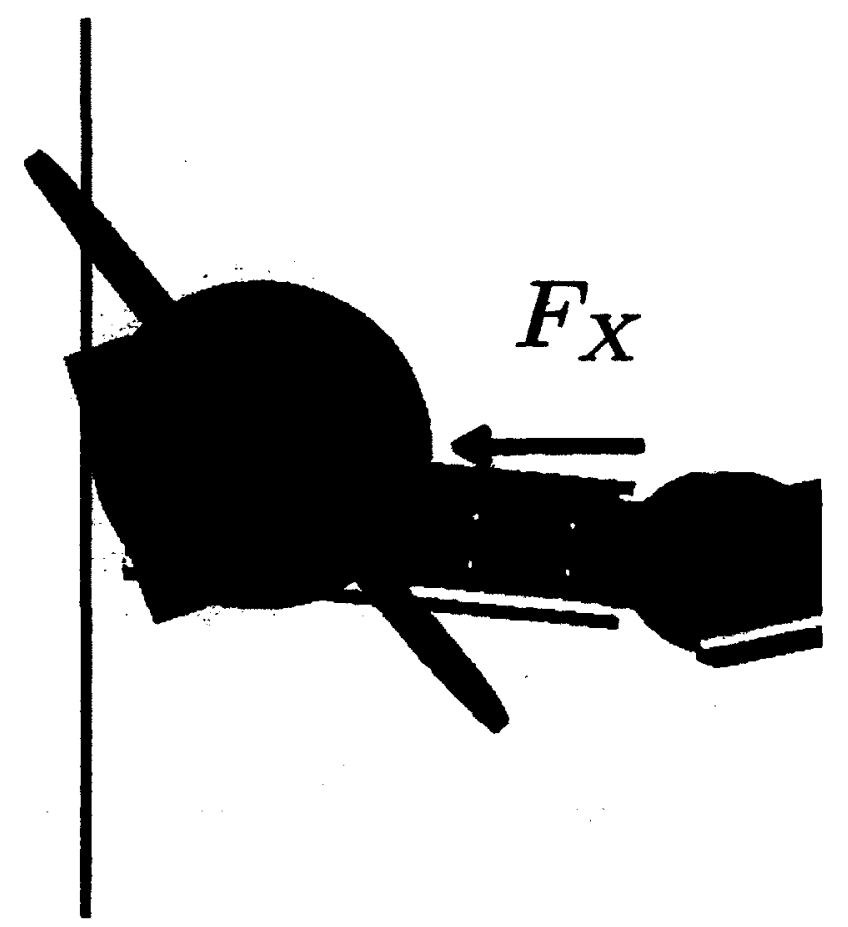

Figure 3.11: Visualization of the experimental setup to test varying $K$ and $D$ with the haptic rendering algorithm. 

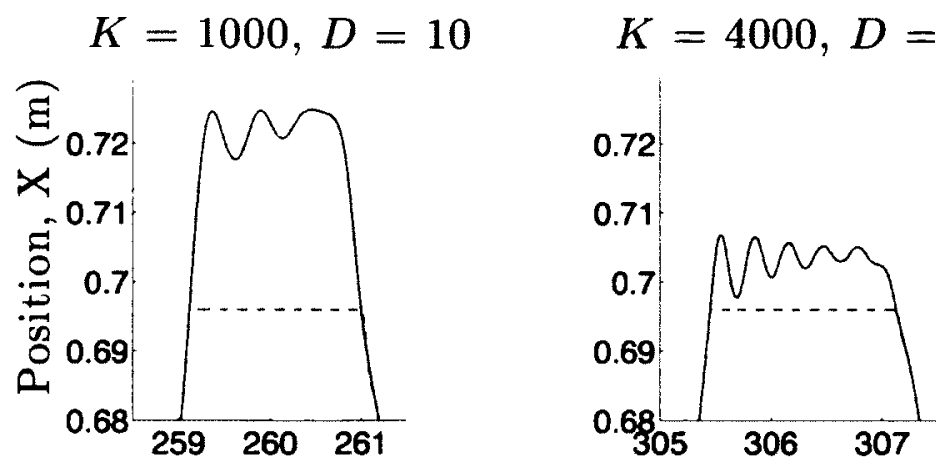

$$
K=4000, D=100
$$
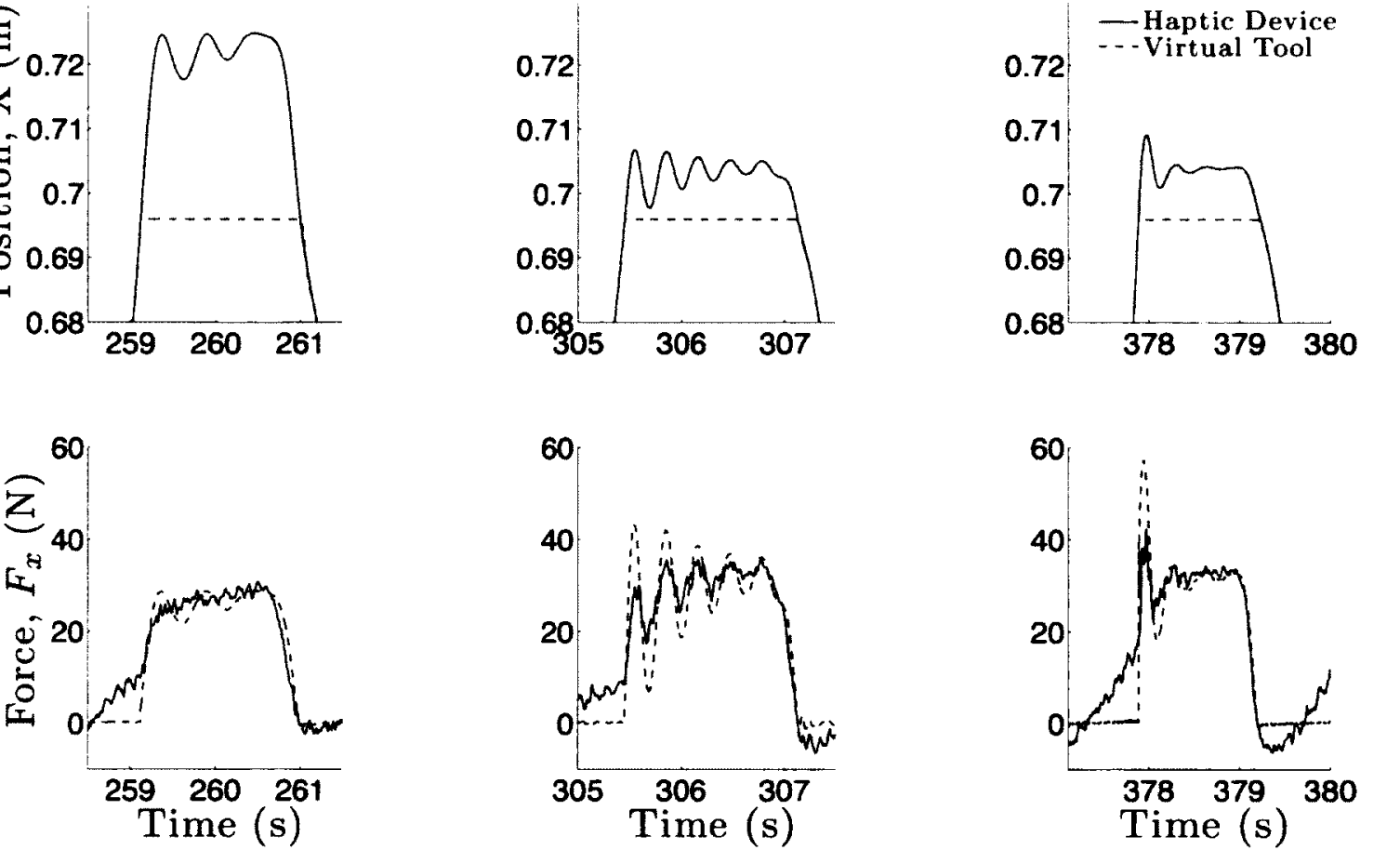

Figure 3.12: Position and force associated with a virtual wall contact in the $X$ direction for varying contact parameters.

The virtual tool can move unconstrained until it makes contact with the wall at $X=0.72$ meters. It is obvious to say that as the spring constant $K$ increases, the haptic device will have a smaller penetration depth for an equal amount of force. Also, as the damping increases, the oscillations seen after penetration decrease.

\subsubsection{Friction Test}

A similar test was conducted to ensure friction forces can be felt by the user. Similar to the previous experiment, the foot plate was grasped by the user and a force applied to move the haptic device to the virtual wall. Once contact was made, the user attempted to apply a constant force in the $+X$ direction, while simultaneously moving the haptic device in the $+Y$ and $-Y$ directions as seen in Figure 3.13. Without friction, the virtual tool slides along the surface of the virtual wall without a force opposing the motion as seen in the 
lefthand graphs of Figure 3.14 below. However, with friction applied $(\mu=0.3)$, the force due to friction $F_{y}$ is a function of the normal force $F_{x}$ and the Coulomb friction as can be seen in the righthand graphs of Figure 3.14. Also, with friction applied, a desired torque is generated from the virtual object being stuck to the surface of the wall.

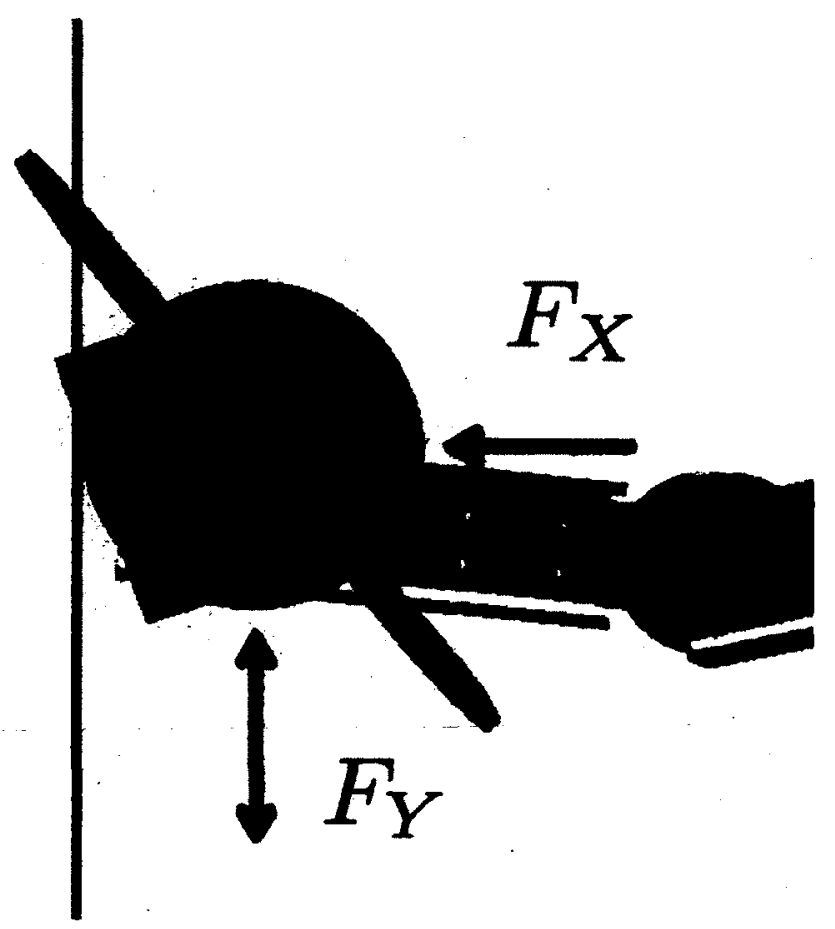

Figure 3.13: Visualization of the experimental setup to test friction with the haptic rendering algorithm. 

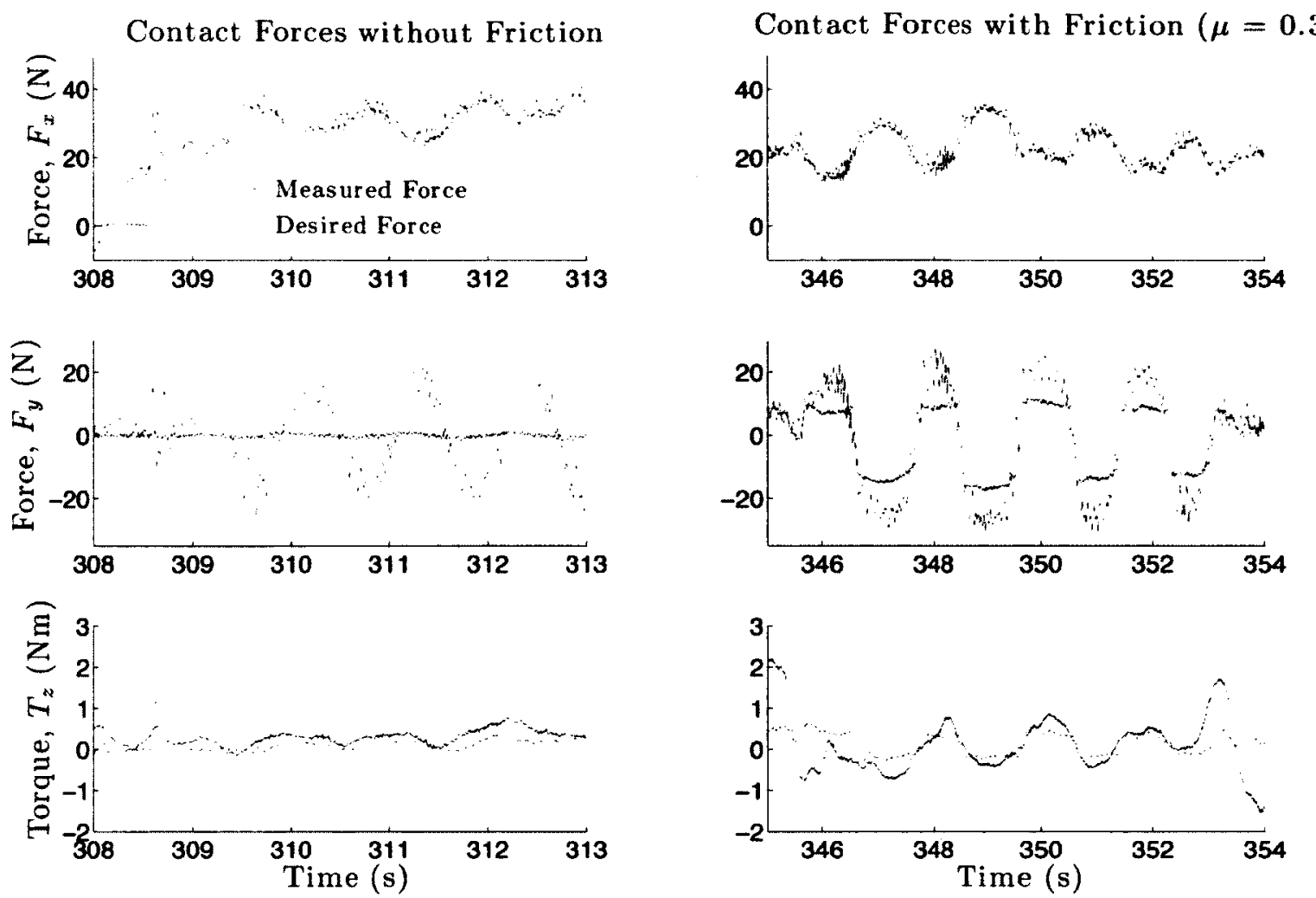

Figure 3.14: Measured and desired forces and torques for a virtual wall contact test with and without friction.

\subsubsection{Box Test}

A more complex test was conducted in order to test the stability of the haptic rendering algorithm. Like the previous tests, the hand is used to grasp the foot plate in order to manipulate the robot. The virtual tool is now chosen as a cube which will provide the user with forces in the $X$ and $Y$ directions and a moment about the $Z$ axis. The cube, with dimensions of $0.1 \mathrm{~m} \times 0.1 \mathrm{~m} \times 0.1 \mathrm{~m}$, is dragged along the surface of a wall, around a step, and then inserted into a gap which is the same width as the cube. Figure 3.6.3 shows the sequence of events that occured during the experiment. The results are shown in Figure 3.16 with the dotted red line signifying the position of the virtual tool and the solid blue line signifying the position of the haptic device.

As can be seen in Figure 3.16, during time interval (a), the virtual tool is constrained 


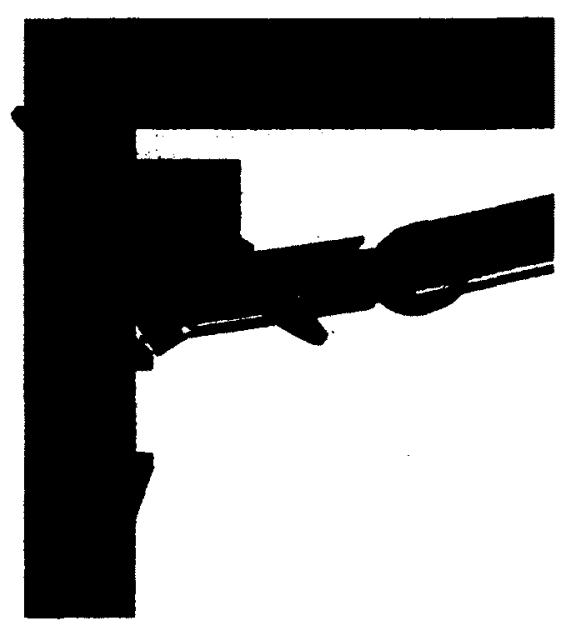

(a) Contact with wall.

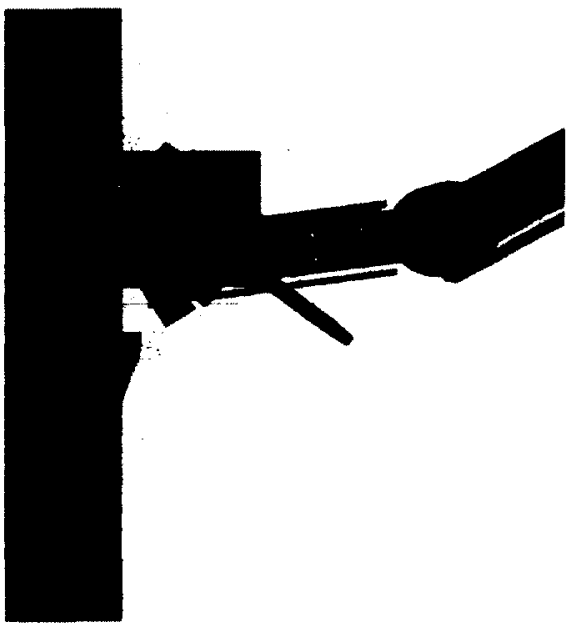

(c) Contact with step.

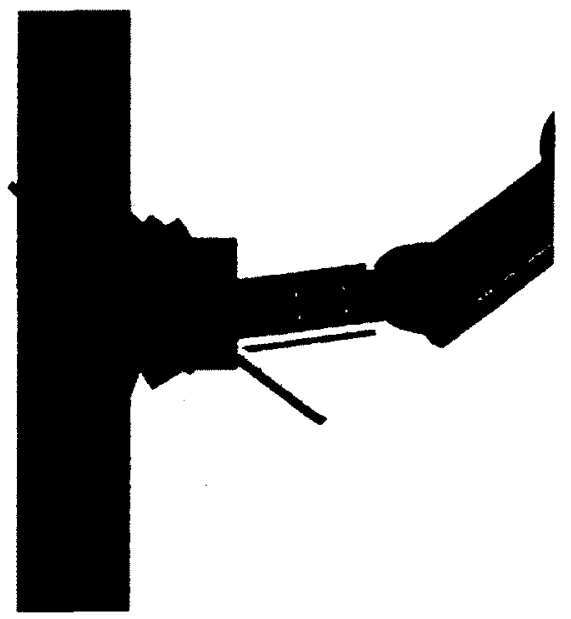

(e) Box fully inserted.

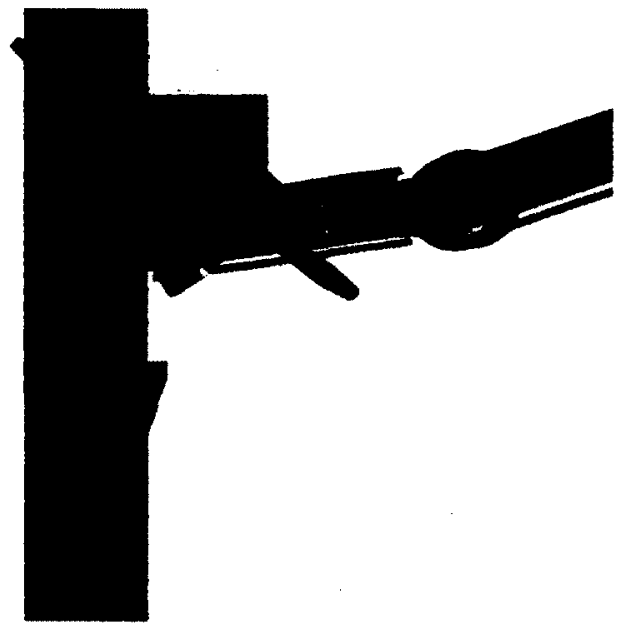

(b) Contact with wall and step.

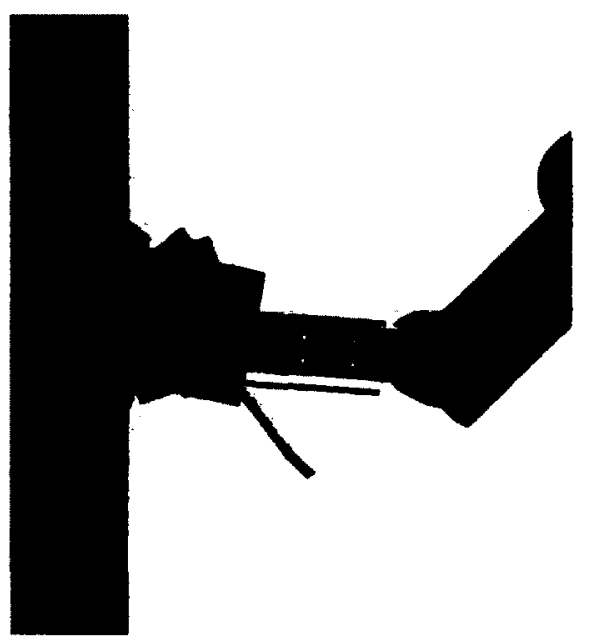

(d) Box misaligned.

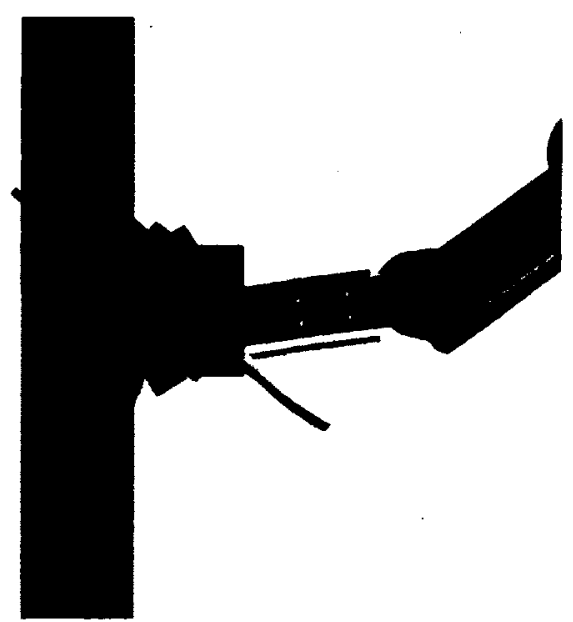

(f) Wiggling the box.

Figure 3.15: Haptic rendering box test for reference with Figure 3.16. 
to the wall at $X=0.72 \mathrm{~m}$. The box is then moved around the step until it is fully inserted at interval (e) where it is constrained to the inside of the gap. The box is wiggled in the $X$ and $Y$ directions to ensure that the rendering maintains a stable contact.
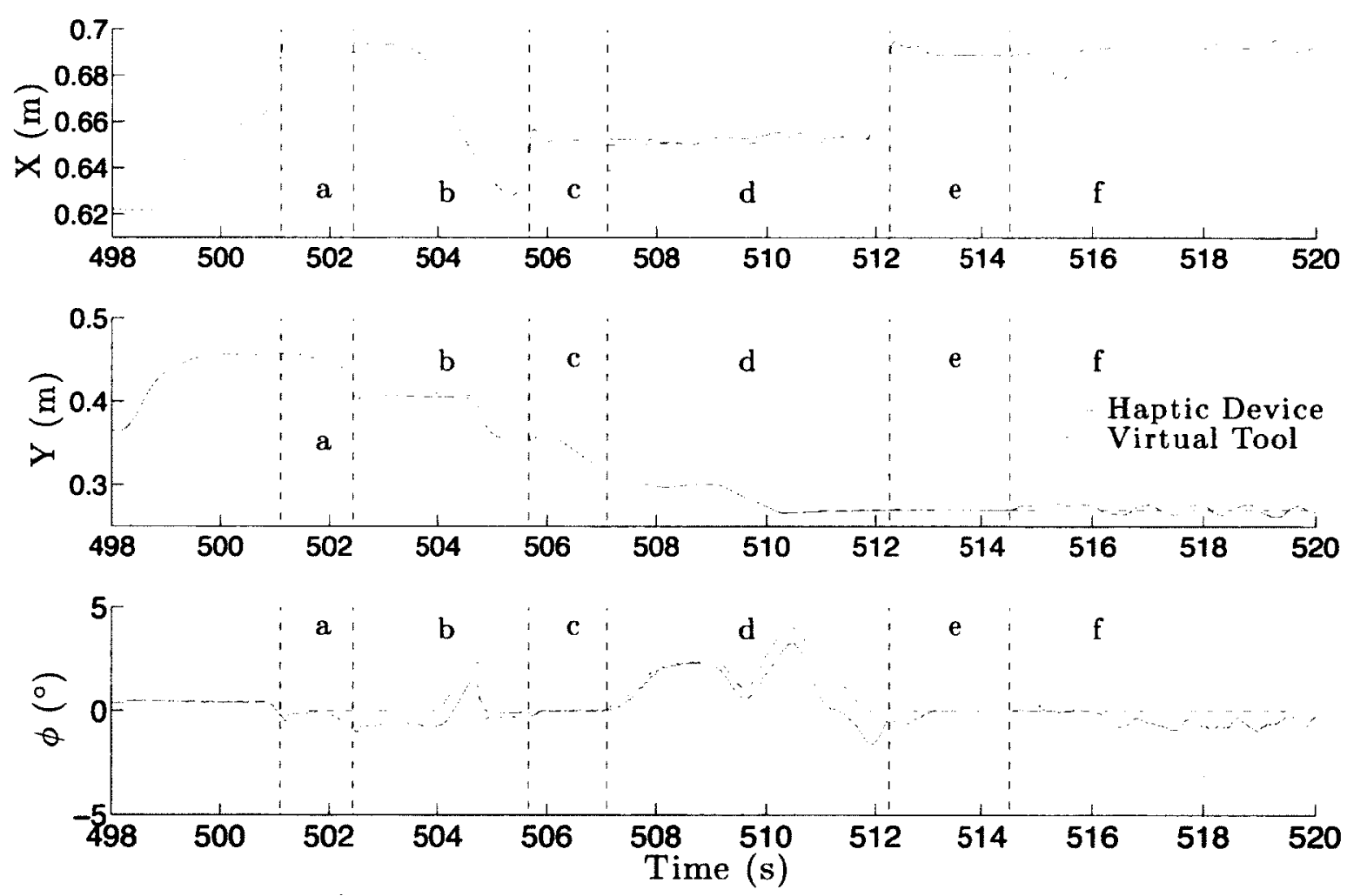

Figure 3.16: Haptic device and virtual tool positions with respect to time. The virtual tool is defined as a box.

\subsection{Haptic Device Performance Measures}

Many haptic devices have been developed for a wide range of purposes. Although there is no standard for comparing the performance of one device to another, Hayward et al. attempt to define a list of such measures [53]. ViGRR is evaluated in this section, using these measures as a guideline. 


\subsubsection{Degrees of Freedom and Classification}

According to Hayward et al., a haptic device can be classified as either low DoF, high DoF, or very high DoF. A low DoF device encompasses any device which cannot address the literal emulation of tasks, usually 2 or 3-DoF. A high DoF device is considered any device which can recreate a task in its integral form, usually 5 or 6-DoF. A very high DoF device not only can recreate a task in its integral form, but can also incorporate body motions such as an exoskeleton. Based on this definition, ViGRR is classified as a low DoF haptic device since it does not fully emulate a task which would occur during actual performance.

\subsubsection{Device-Body Interface}

The device-body interface on most haptic devices constitutes the user grasping the endeffector. The interface for ViGRR is made through a velcro connection of the user's foot to the footplate as seen in Figure 3.17. By this connection, the user's foot has 3 active degrees of freedom through the robot's plane of motion, while the other 3 are constrained.

\subsubsection{Range of Motion}

Due to the redundant kinematics of the ViGRR, any point within the circle defined by the fully outstretched length of the robot can be accessed. Therefore, it is more informative to illustrate the region in which the robot is normally operating. Any point less than $0.3 \mathrm{~m}$ is not considered as that does not fall within the normal operating workspace of ViGRR. Figure 3.18 defines the reachable workspace of ViGRR for an end-effector angle of $0^{\circ}$. The robot base is defined as $X=Y=0 \mathrm{~m}$ 


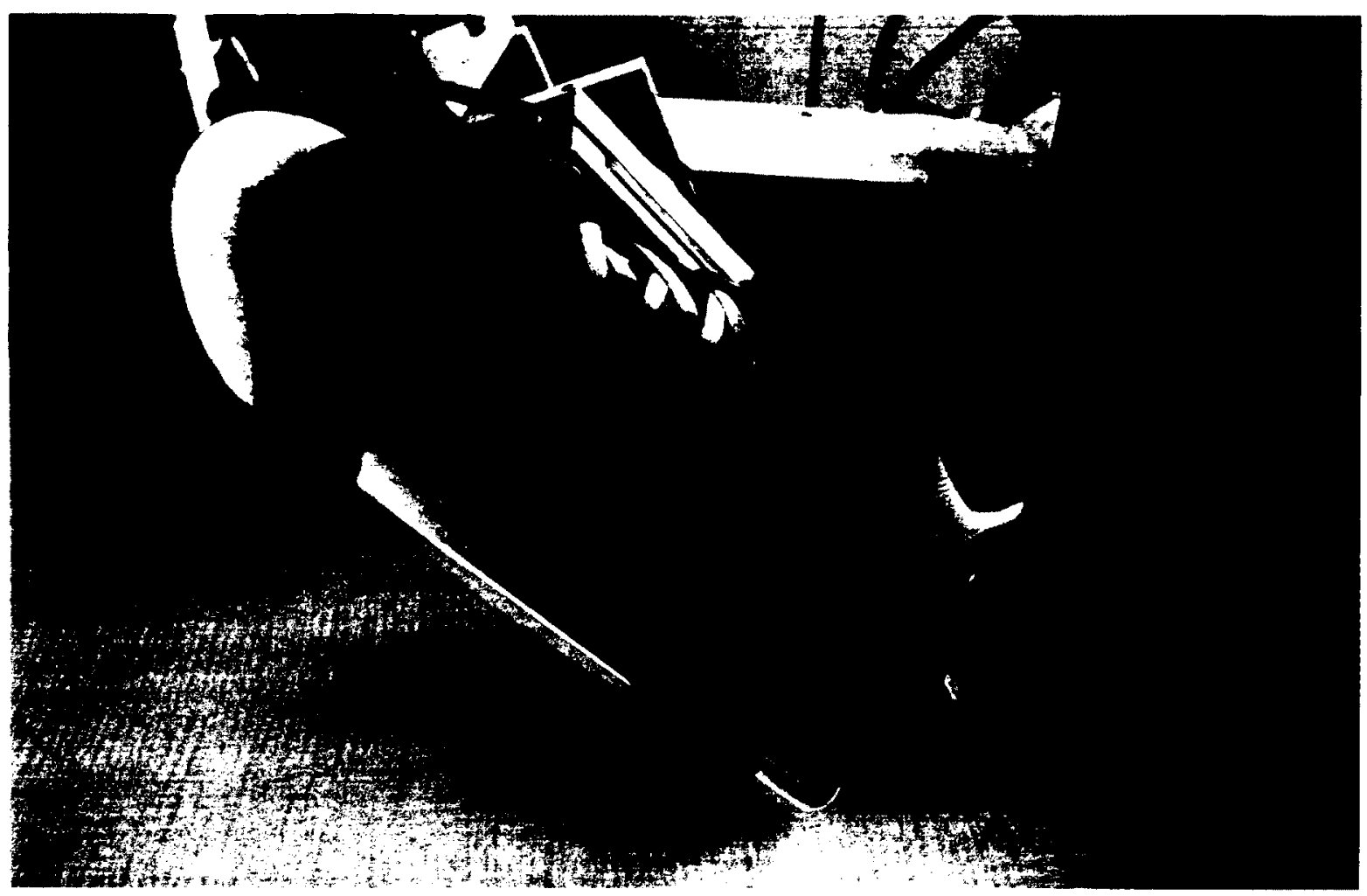

Figure 3.17: The device-body interface of the ViGRR. The user's foot is strapped to the footplate by two velcro strips. 


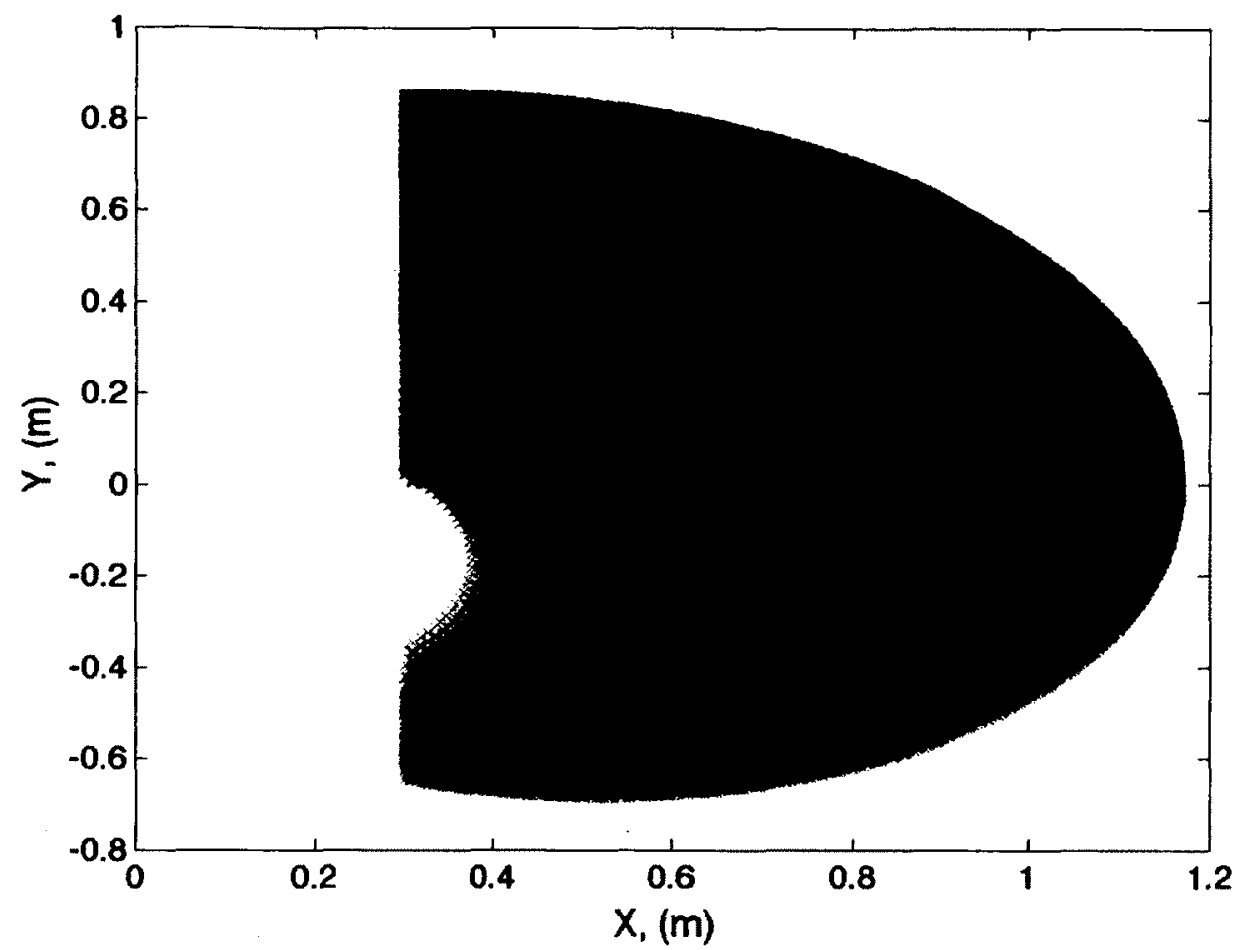

Figure 3.18: Reachable workspace of ViGRR for end-effector angle $\phi=0^{\circ}$. The base of the robot is defined as $X=Y=0 \mathrm{~m}$ and any point behind $0.3 \mathrm{~m}$ is not shown as it is not considered the normal operating range of ViGRR.

\subsubsection{Manipulability Ellipsoid}

One way to evaluate robot manipulator performance is by determining the ability for the robot to arbitrarily change end-effector position and orientation. This capability can be determined by finding the velocity manipulability ellipsoid. The equation

$$
\dot{q}^{T} \dot{q}=1
$$

describes the points on the surface of a sphere in the joint velocity space. The points can then be mapped to the task space through the equation $\dot{q}=J(q)^{-1} v_{e}$ which yields

$$
v_{e}^{T}\left(J(q) J^{T}(q)\right)^{-1} v_{e}=1
$$

where $J$ is the Jacobian and $v_{e}$ is the end-effector velocity in task space. This equation 
is derived for non-redundant manipulators, however the redundant case yields the same result [54]. Equation (3.17) describes the points on the surface of an ellipsoid in the endeffector velocity space. Along the direction of the ellipsoids major axis, the end-effector can move at large velocities, as opposed to the small velocities obtained in the direction of the minor axis. Also, the more spherical the ellipsoid is, the better the end-effector is at moving along any direction. The eigenvectors of $J(q) J^{T}(q)$ define the direction of the principal axes of the ellipsoid whereas the dimensions are defined by the eigenvalues. Figure 3.19 below shows several configurations of the robot and their corresponding velocity manipulability ellipses.

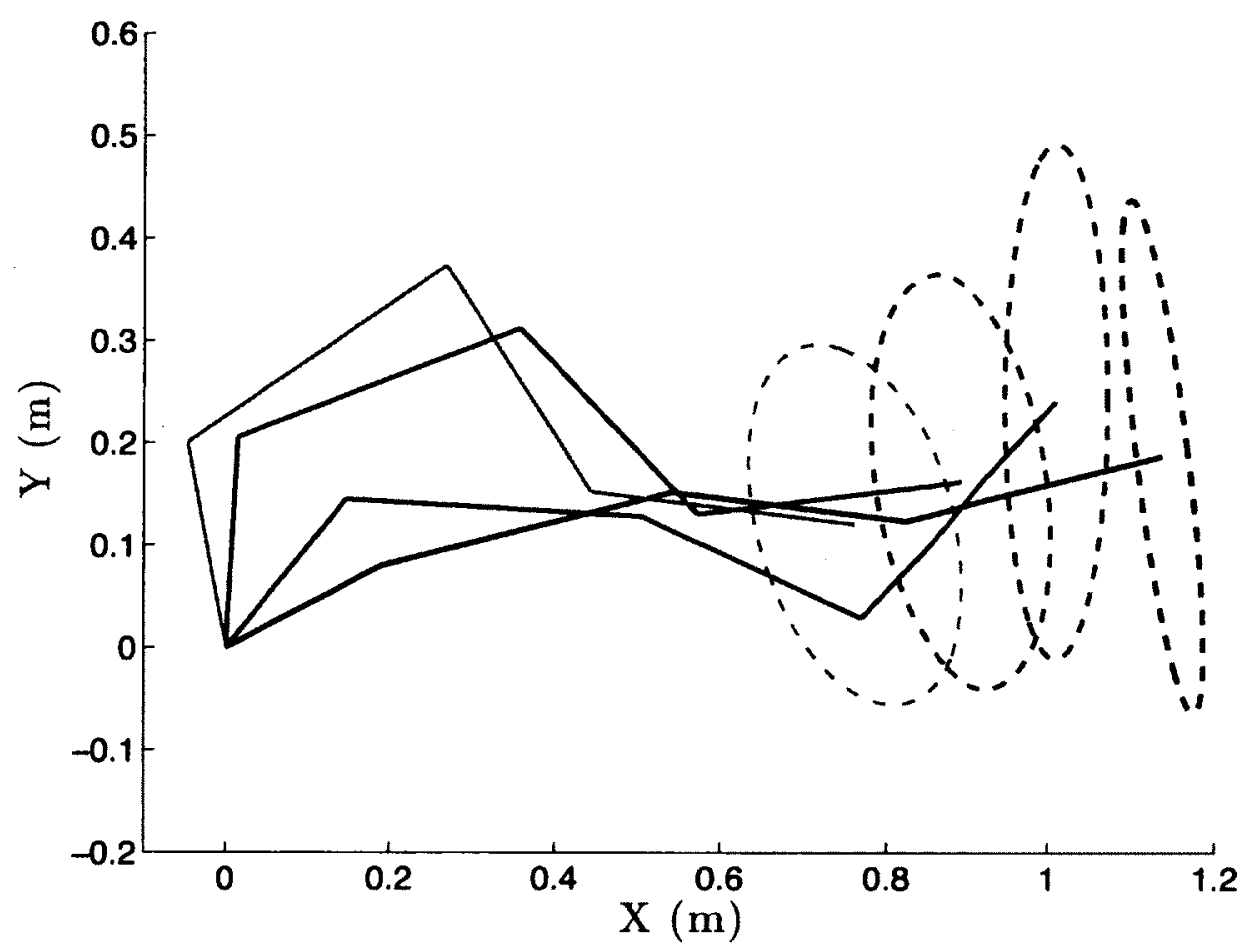

Figure 3.19: Velocity manipulability ellipsoids for ViGRR at different joint configurations.

It is sometimes hard to quantify the areas of poor manipulability based on an ellipsoid. In order to visualize this quantity, a global measure of manipulation ability can be obtained by calculating the volume of the ellipsoid. This volume, termed the manipulability measure, 
can be calculated through

$$
w(q)=\sqrt{\operatorname{det}\left(J(q) J^{T}(q)\right)}
$$

The manipulability measure $w(q)$ of ViGRR is mapped through the same workspace as described above and can be seen in Figure 3.20 below. It is ideal to operate the robot in regions of high manipulability in order to achieve the best performance.

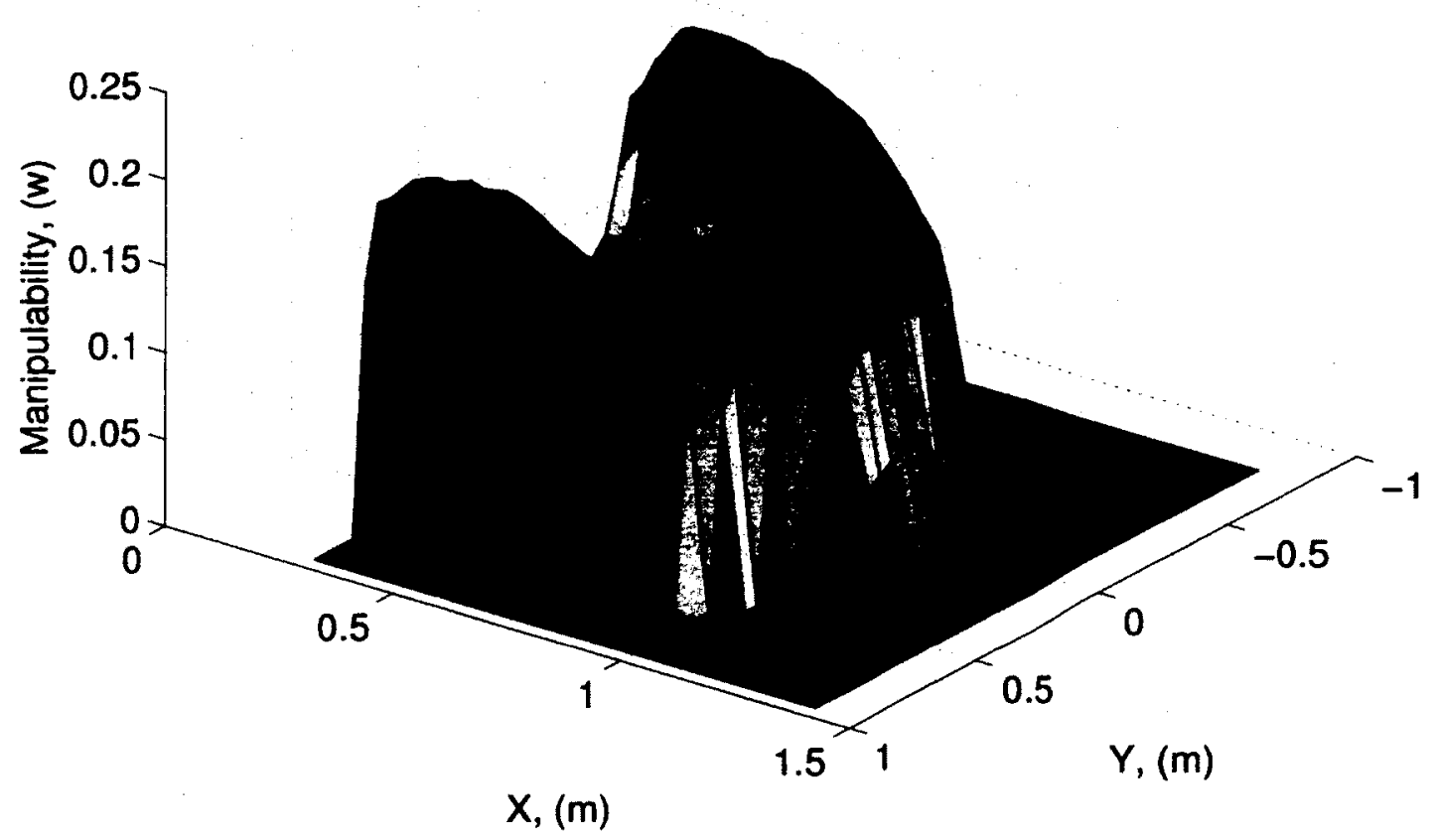

Figure 3.20: Global manipulability measure within the ViGRR workspace for an endeffector angle $\phi=0^{\circ}$.

There exists a duality between velocity and force which shows that the principal axes of the force manipulability ellipsoid coincide with the principal axes of the velocity manipulability ellipsoid but with the dimensions inverted. Therefore, if a robot can move along a direction with high velocity manipulability, it will have low force manipulability and vice 
versa. Although this measure is not suitable for direct comparison between two devices, it does serve the purpose of illustrating the ideal position of the links to execute a specific task. Figure 3.21 below illustrates the duality of the force and velocity manipulability ellipsoids.

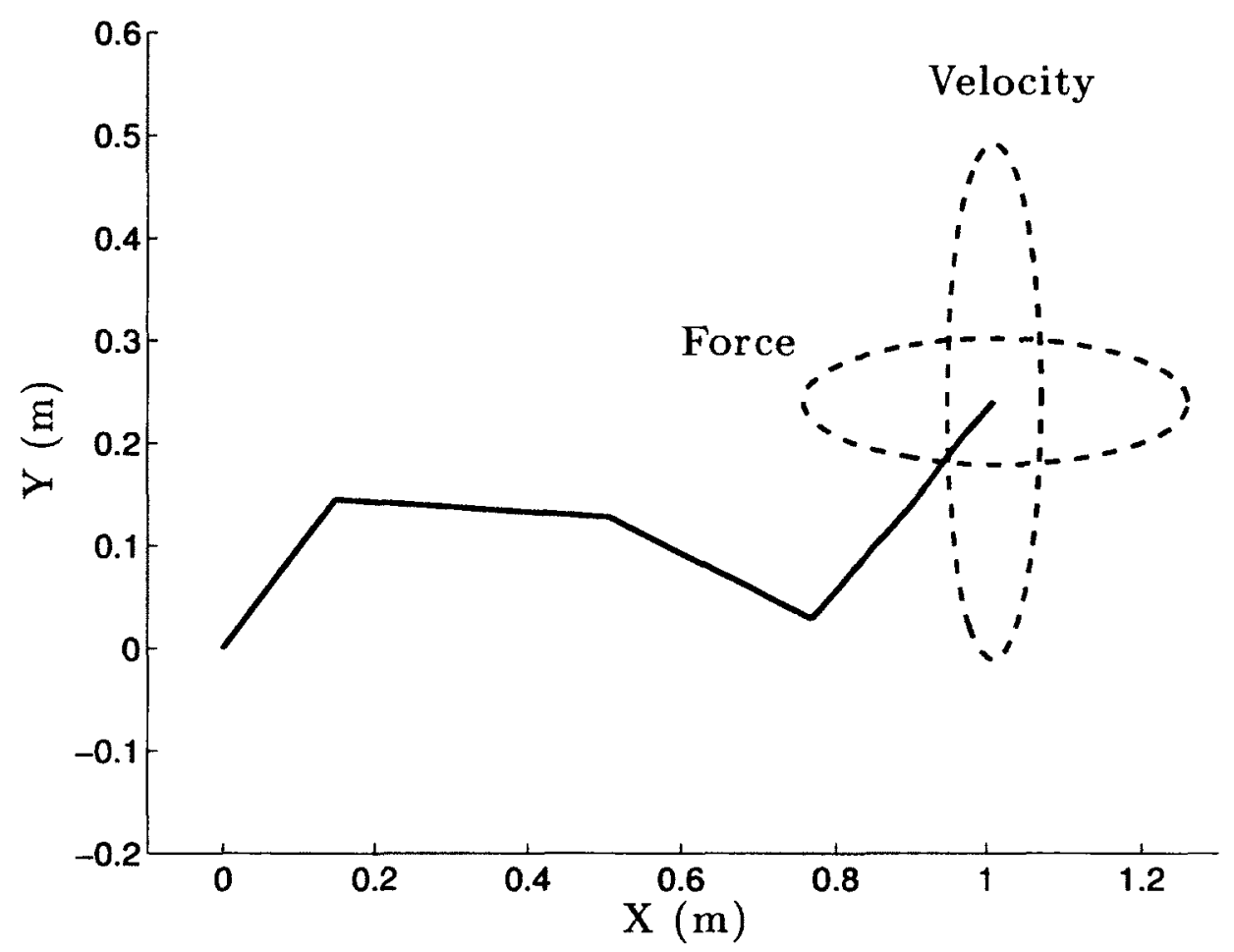

Figure 3.21: Force and velocity manipulability ellipses for a single configuration of ViGRR

\subsubsection{Mass and Damping}

For a haptic device controlled in admittance mode, the mass and damping that can be successfully displayed without instability must be measured. Admittance controlled haptic devices have a physical limit to the lowest inertia that can be displayed. An experiment was conducted where a circle trajectory of radius $0.1 \mathrm{~m}$ is displayed to the user as seen in Figure 3.22. The user attempts to follow this trajectory with a period of 2.5 seconds. Figure 3.23 shows the forces necessary to follow this circle trajectory for a varied mass $M$ and $B$ in the $X$ and $Y$ directions. However, it can be seen that as the device becomes more transparent, it becomes more susceptible to variations in the input force. A minimum 
value of $M=6 \mathrm{~kg}$ was achievable with the ViGRR platform as any value lower than that produces unstable behavior.
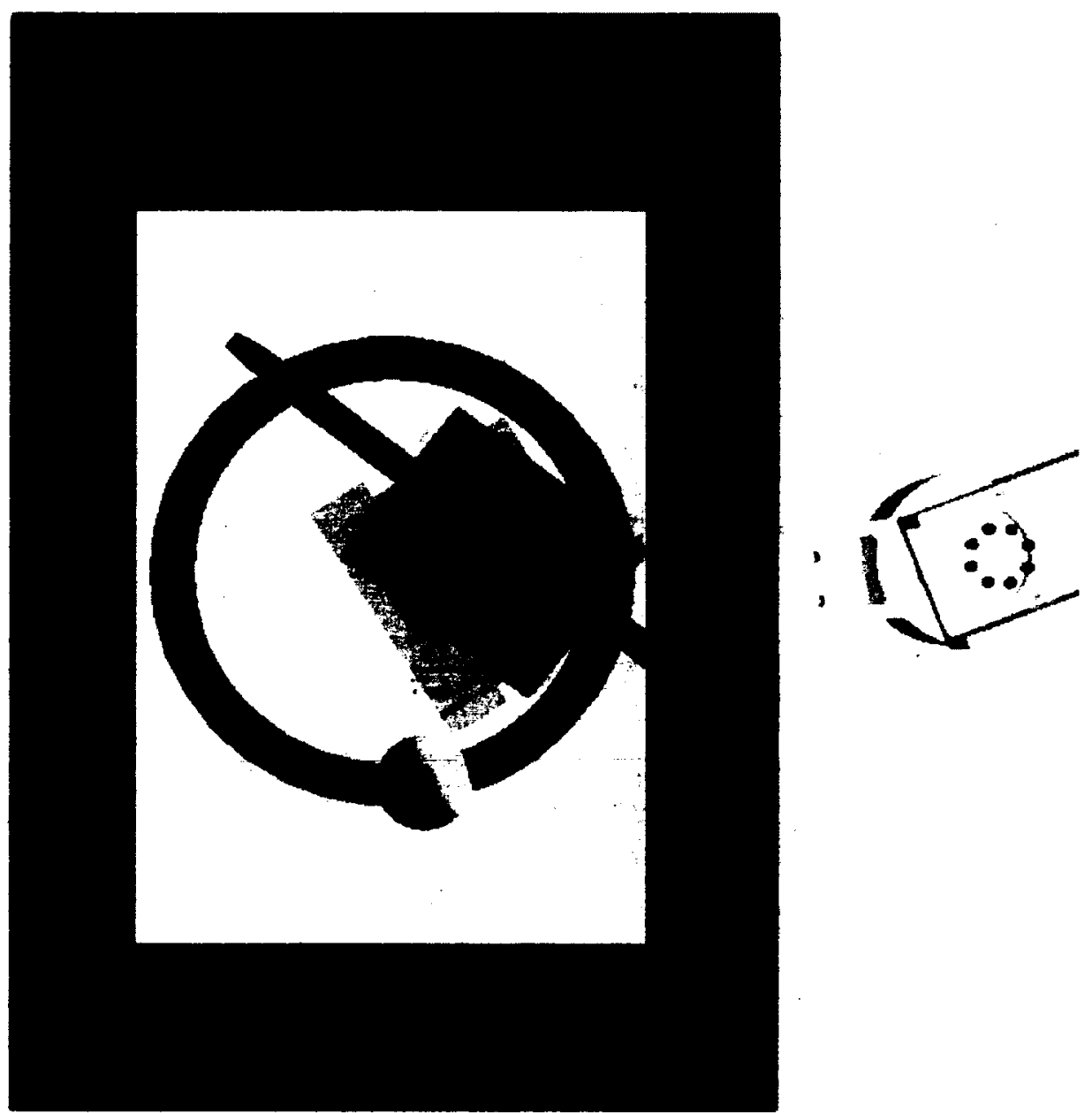

Figure 3.22: Circle trajectory for admittance controlled experiments. The yellow ball represents the trajectory to be followed and the red ball represents the virtual tool of the device.

A similar test was conducted with the $\phi$ direction and can be seen in Figure 3.24 below. The moments seen here are measured while following a rotational trajectory of $45^{\circ}$ with the same period as before.

The lowest achievable admittance parameters with a hand coupling can be seen in Table 3.1. The FT deadband column on the right corresponds to the minimum force or torque required to produce a position output. This deadband must be present in order to 

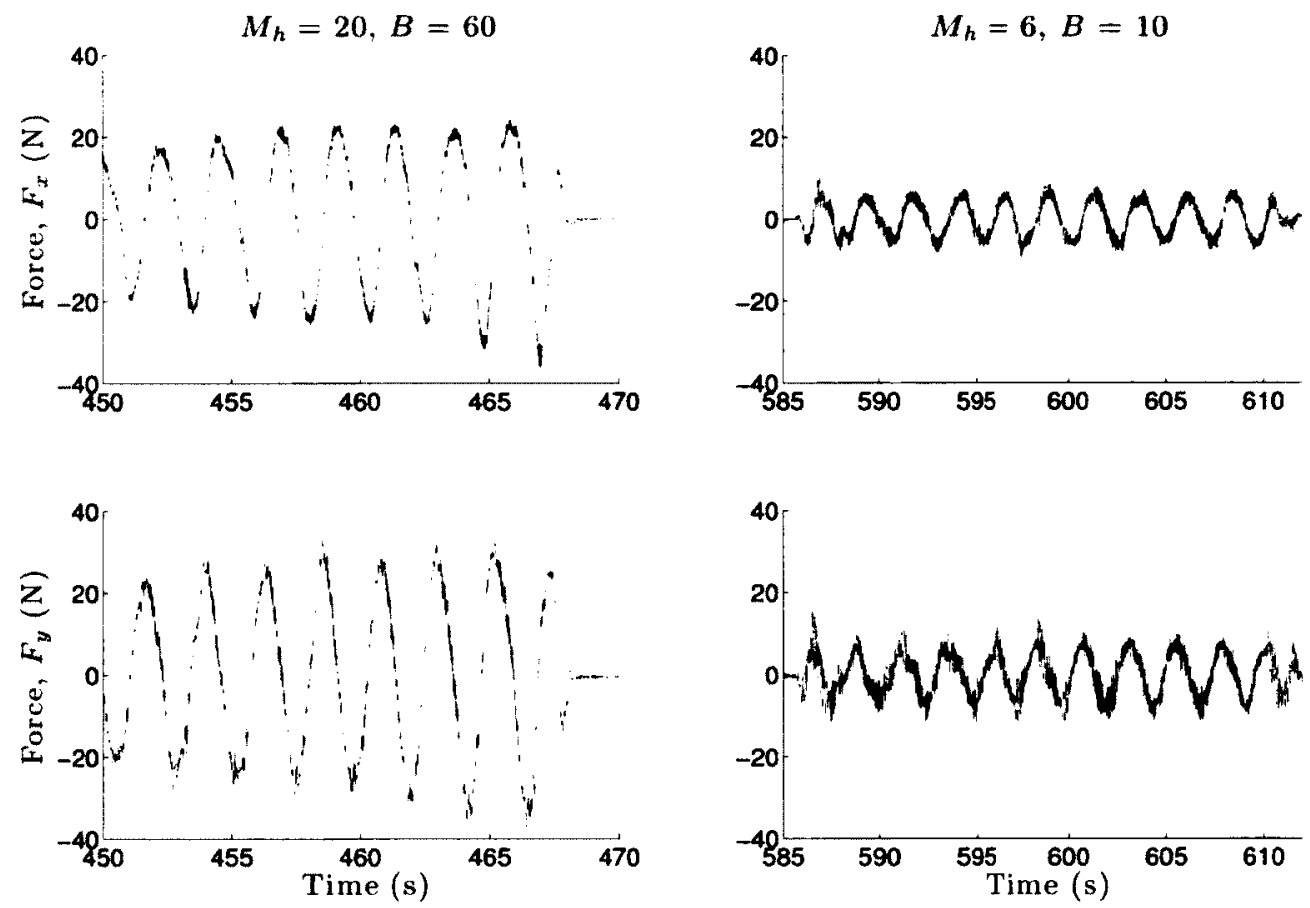

Figure 3.23: Forces in the $X$ and $Y$ directions while following a circle trajectory of $r=$ $0.1 \mathrm{~m}$ and $T=2.5 \mathrm{~s}$.
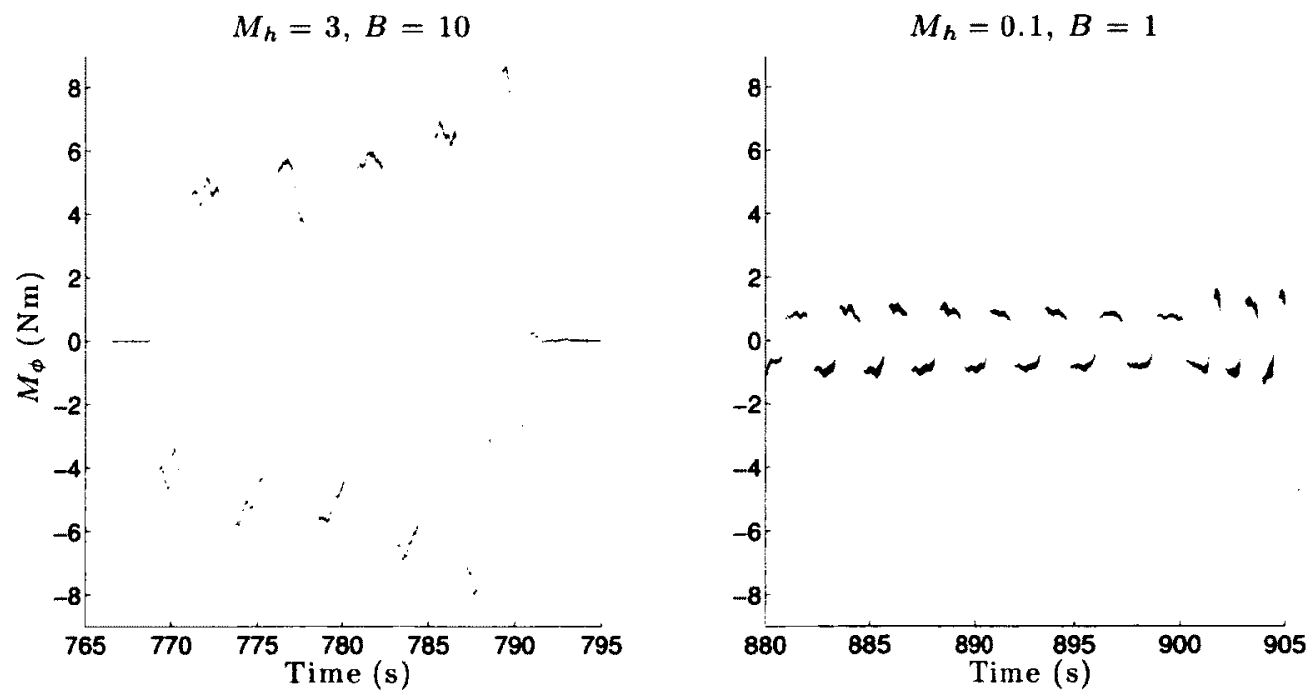

Figure 3.24: Moment in the $\phi$ direction while following a rotation trajectory of $45^{\circ}$ and $T=2.5 \mathrm{~s}$. 
filter any noise which is inherent in the sensor.

Table 3.1: Mass and Damping Parameters of ViGRR in Admittance Control for Hand Coupling

\begin{tabular}{|c|c|c|c|}
\hline & Mass $\left(M_{h}\right)$ & Damping $(B)$ & FT Deadband \\
\hline $\mathrm{X}$ & $6 \mathrm{~kg}$ & $10 \frac{\mathrm{Ns}}{\mathrm{m}}$ & $1 \mathrm{~N}$ \\
\hline $\mathrm{Y}$ & $6 \mathrm{~kg}$ & $10 \frac{\mathrm{Ns}}{\mathrm{m}}$ & $1 \mathrm{~N}$ \\
\hline$\phi$ & $0.1 \mathrm{kgm}^{2}$ & $1 \frac{\mathrm{Nms}}{\mathrm{rad}}$ & $0.2 \mathrm{Nm}$ \\
\hline
\end{tabular}

\subsubsection{Resolution}

The resolution of ViGRR can be defined by the smallest measurable displacement in task space. The task space resolution is dependant on the encoder resolution of each joint as well as the configuration of the robot. The task space resolution is mapped out for the operating workspace of ViGRR in Figure 3.7.6 below. The angular resolution of ViGRR in task space is simply the sum of all four encoder resolutions. The first two motors have an angular resolution of 6250 pulses/rev and the last two have a resolution of $4096 \mathrm{pulses} / \mathrm{rev}$. Table 3.2 below outlines the task space and joint space resolution of the robot as well as the resolution of the FT sensor. 


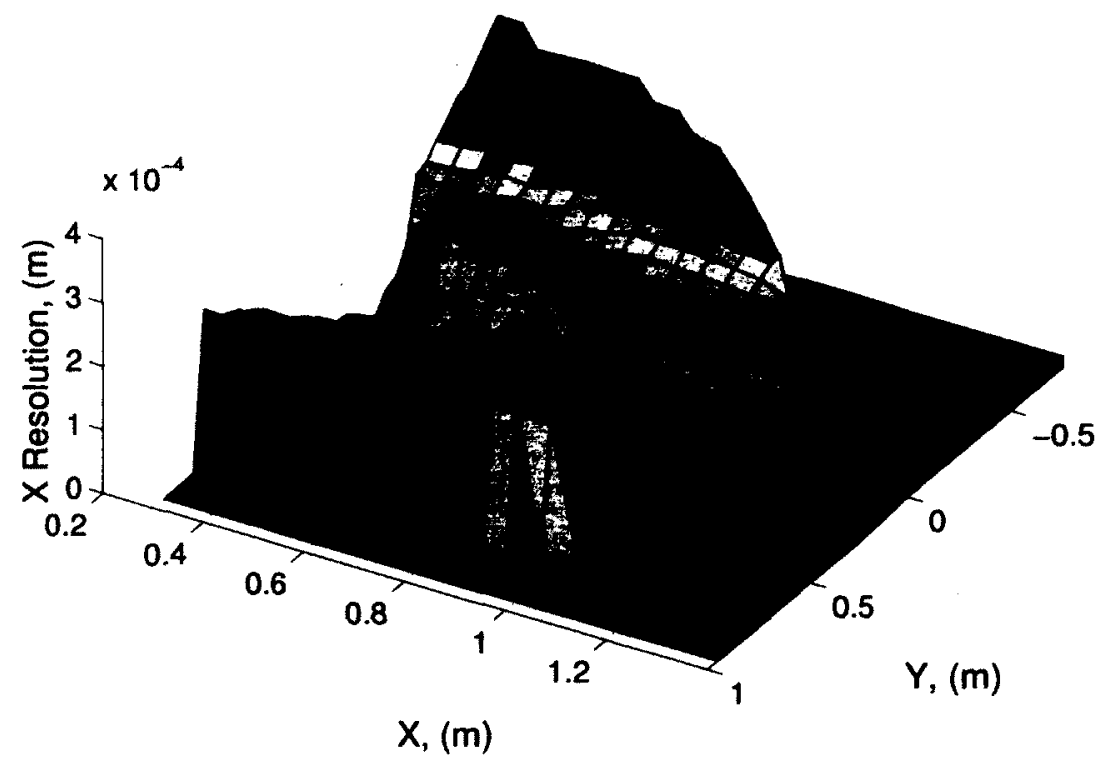

(a)

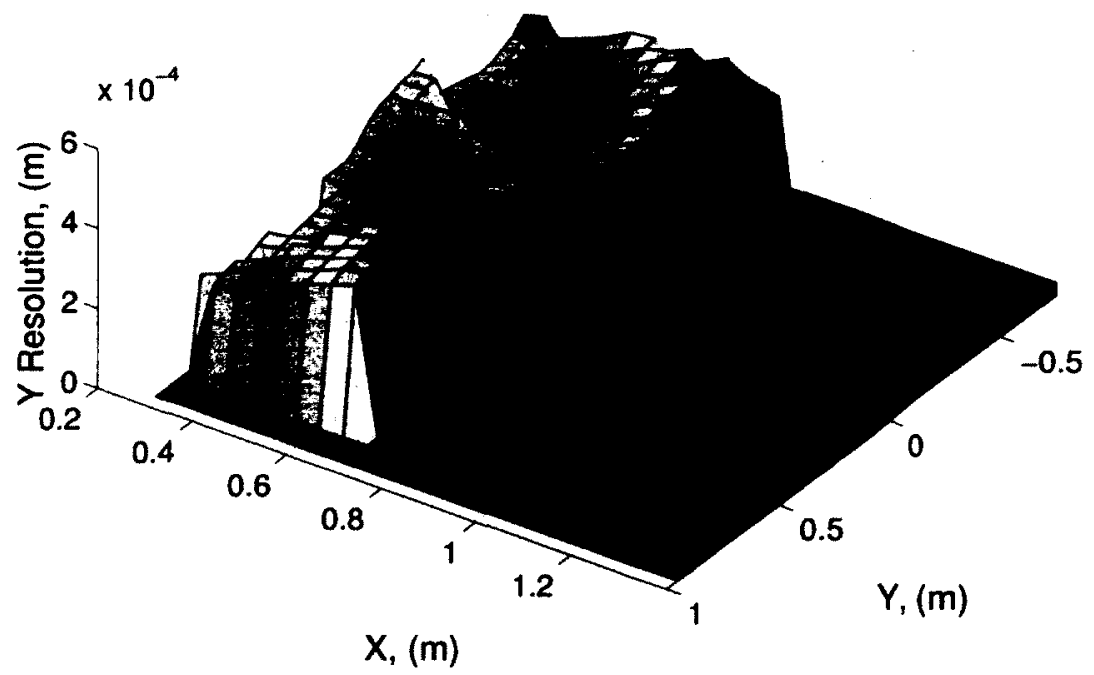

(b)

Figure 3.25: Task space resolution of ViGRR in $X$ and $Y$ directions depending on the position of the end-effector. 
Table 3.2: ViGRR Resolution

\begin{tabular}{|c|c|c|}
\hline & 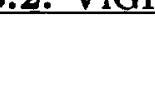 & Resolution \\
\hline FT Sensor & $\begin{array}{c}F_{X}, F_{Y} \\
F_{Z} \\
T_{X}, T_{Y} \\
T_{Z}\end{array}$ & $\begin{array}{c}9 / 28 \mathrm{~N} \\
3 / 7 \mathrm{~N} \\
5 / 374 \mathrm{Nm} \\
7 / 748 \mathrm{Nm}\end{array}$ \\
\hline Task Space & $\begin{array}{l}X \\
Y \\
\phi\end{array}$ & $\begin{array}{c}0.3119 \times 10^{-3} \mathrm{~mm} \\
0.5606 \times 10^{-3} \mathrm{~mm} \\
0.2910^{\circ}\end{array}$ \\
\hline Joint Space & $\begin{array}{l}1 \\
2 \\
3 \\
4\end{array}$ & $\begin{array}{l}0.0576^{\circ} \\
0.0576^{\circ} \\
0.0879^{\circ} \\
0.0879^{\circ}\end{array}$ \\
\hline
\end{tabular}

\subsubsection{Precision and Repeatability}

The precision of ViGRR can be determined by using an external measurement device to verify the ability of the robot encoders to determine the pose of the end-effector. For our purposes, the Optotrak measurement system (described in Chapter 2) was used and the precision over a set of 100 data points gave a mean error of $2.8576 \mathrm{~mm}$.

The repeatability of the robot was found by commanding the robot to move to four corners of a box within its normal operating space 15 times each. This will provide 4 separate repeatability measures, one for each corner of the box. The repeatability measurements of the robot were found using an ISO standard for industrial manipulators with a $99.8 \%$ confidence level $( \pm 3 \sigma)$ [55]. This means that when the robot is commanded to a specified position, $99.8 \%$ of the time it falls within a radius defined by the repeatability range. The 
results are shown in Table 3.3 .

Table 3.3: Repeatability Range for 4 End-Effector Positions

\begin{tabular}{|c|c|c|}
\hline$X$ Position (mm) & $Y$ Position (mm) & Repeatability Range (mm) \\
\hline 600 & 600 & 0.1109 \\
\hline 800 & 200 & 0.2000 \\
\hline 800 & 600 & 0.1654 \\
\hline 600 & 200 & 0.0640 \\
\hline
\end{tabular}

\subsection{Summary}

This chapter addresses the haptic development of the ViGRR platform. To start, a set of haptic requirements were defined which outline the various features that are essential to the fidelity of lower limb haptics. These requirements include 6-DoF rendering, friction, variable contact parameters, and real-time QNX compatibility. A haptic rendering approach was then chosen based on those requirements which involved the integration of a rigid body simulation tool, Open Dynamics Engine. This haptic rendering approach was then implemented on a real-time QNX system and evaluated. From the evaluation tests, it is shown that the haptic rendering algorithm satisfies the requirements outlined at the beginning of this chapter. The end of this chapter details a list of measures that can be used to compare the haptic performance of ViGRR with other similar devices.

Now that ViGRR has been proven to function as a haptic device, it is now possible for ground reaction force experiments to be conducted with a lower limb. 


\section{Chapter 4}

\section{Lower Limb Haptic Rehabilitation with ViGRR}

The previous chapters outlined the hardware improvements necessary for ViGRR to function as a lower limb haptic robot. They also described the development and implementation of a haptic rendering algorithm that will be used to produce feedback forces for use with gait training and other rehabilitation exercises. The ViGRR platform is now ready to be developed as a lower limb haptic rehabilitation robot.

The experiments described below were chosen in order to highlight the main advantages of ViGRR compared to other lower limb rehabilitation robots. These experiments are divided into two sections: gait experiments and virtual reality exercises.

\section{Gait Experiments}

In order to understand some of the concepts related to these experiments, a summary of gait fundamentals are provided at the beginning of this chapter. As will be described below, the gait cycle can be divided into two phases, swing and stance. The experiments presented in this section were designed to show the ability of VIGRR to reproduce each of these phases. During the swing phase, the human leg is propelled through the air with no contact from the environment. Therefore, the swing phase experiments determine the ability of ViGRR to move with as little force input as possible, or in other words, the transparency of ViGRR. Conversely, the stance phase constitutes any instance in which the human leg is in contact with the ground. The stance phase experiments assess the ability of ViGRR to reproduce ground reaction 
forces based on a normal gait trajectory.

\section{Virtual Reality Exercises}

A set of virtual reality exercises are developed which highlight the broad range of activities that ViGRR can perform with regards to rehabilitation. A human subject participated in each experiment in order to provide feedback regarding the therapeutic merit of the exercise.

\subsection{Gait Fundamentals}

The term gait refers to the pattern of movement of the limbs of a human during locomotion. Often, the term normal gait is used to describe the gait of healthy subjects. However, a normal gait for a woman over the age of 55 is dramatically different from the normal gait of a man of age 25. The term normal must then be a function of the patient's sex, age, weight, and body geometry. When looking at the gait for a particular subject, these parameters must be taken into consideration to find the appropriate normal [20].

\subsubsection{Gait Cycle}

The gait cycle can be broken down into seven major events: initial contact, opposite toe off, heel rise, opposite initial contact, toe off, feet adjacent, and tibia vertical. These seven events occur during the two phases of the gait cycle: stance and swing. Stance phase occurs when the foot is in contact with the ground and swing phase occurs when the foot is in motion through the air. The stance phase can be subdivided into: loading response, mid-stance, terminal stance, and pre-swing. The swing phase can be subdivided into: initial swing, mid-swing, and terminal swing. All events and their groups can be more appropriately seen in Figure 4.1.

Figure 4.2 shows the timing diagram from initial contact to toe off for both feet during a normal gait cycle. As can be seen, right initial contact occurs when the left foot is still in contact with the ground. This period is considered double support and occurs until the opposite toe off event. After opposite toe off, there is a period of single support where only 


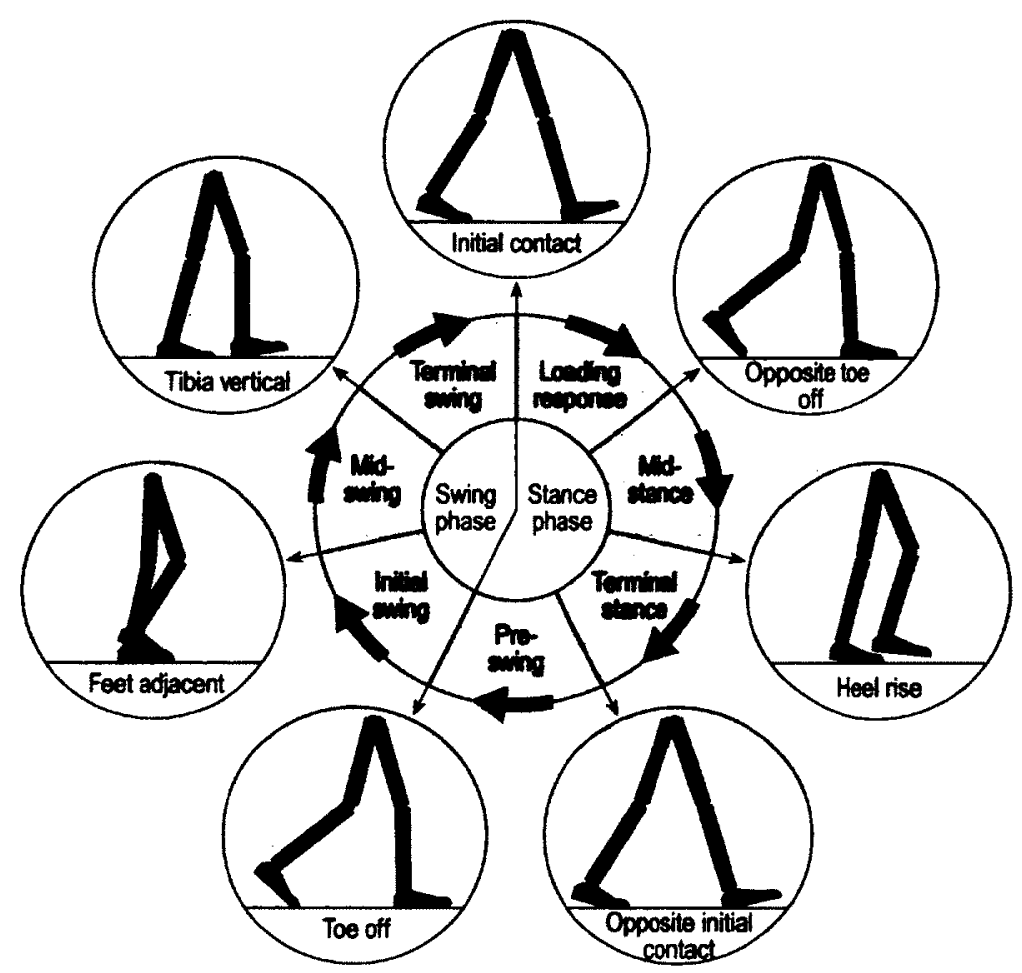

Figure 4.1: Position of the right leg (grey) during a gait cycle [20].

the right foot is in contact with the ground. Ususally, the stance phase lasts $60 \%$ of the gait cycle and the swing phase $40 \%$. The double support period occupies $10 \%$ of the cycle.

Walking speed depends on two parameters: stride length and cadence:

Stride length is the distance between two successive placements of the same foot. The stride length measured from one foot must be equal to the stride length measured from the opposite foot for straight walking.

Cadence is the number of steps taken in a given time (eg. steps $/ \mathrm{min}$ ). In some literature, cadence has been replaced by its inverse known as cycle time, stride time, or stride period (eg. $0.8 \mathrm{~s} / \mathrm{step}$ ). Either way, if both the stride length and cadence or stride time are known, then the speed of walking can be calculated.

\subsubsection{Ground Reaction Forces}

The total force applied by the foot to the ground during the stance phase of one foot is called the ground reaction force (GRF). This force is usually measured through a forceplate 


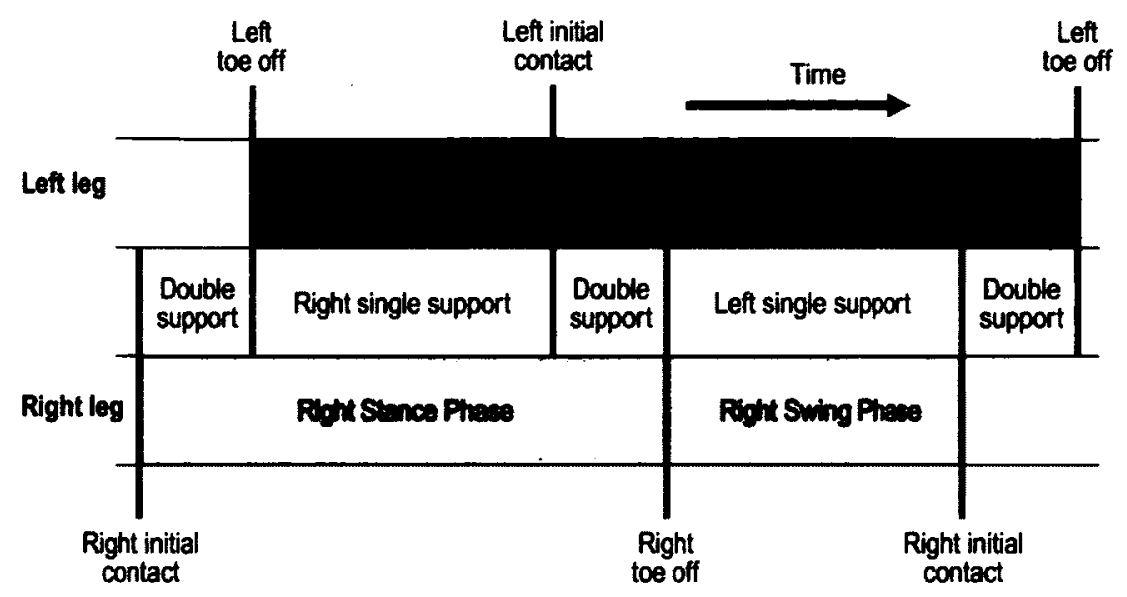

Figure 4.2: Single and double support timing during a gait cycle [20].

and will capture a 3-dimensional force vector with respect to time. This force vector can then be manipulated to yield the vertical, fore-aft (antero-posterior), and lateral (mediolateral) ground reaction forces. Figure 4.3 shows a plot of the three ground reaction forces with respect to the gait cycle for both feet. The lateral component of the force is small, however during the right stance phase there is a slight force which directs the body to the left side and vise-versa. The fore-aft forces during a gait cycle show a braking force during the initial contact and a propulsion force on the second half of the stance phase. The vertical component of the force displays a characteristic 'double hump' which comes from the patient accelerating their centre of mass upward during the early stance phase. This is sometimes referred to as weight acceptance. Next there is a reduction in force as the body passes over the stance leg, and then an increase in force as the toe accelerates the body in late stance phase.

If the sample rate of the forceplate is high enough, a heelstrike transient in the vertical force can be observed at the beginning of the stance phase (Figure 4.4). However, the ground reaction forces are commonly filtered to produce a smooth profile as seen in Figure 4.3 .

The forces measured by the forceplate can also be used to measure the centre of pressure $(\mathrm{CoP})$ of the foot. The CoP is the point on the ground in which a single resultant force 


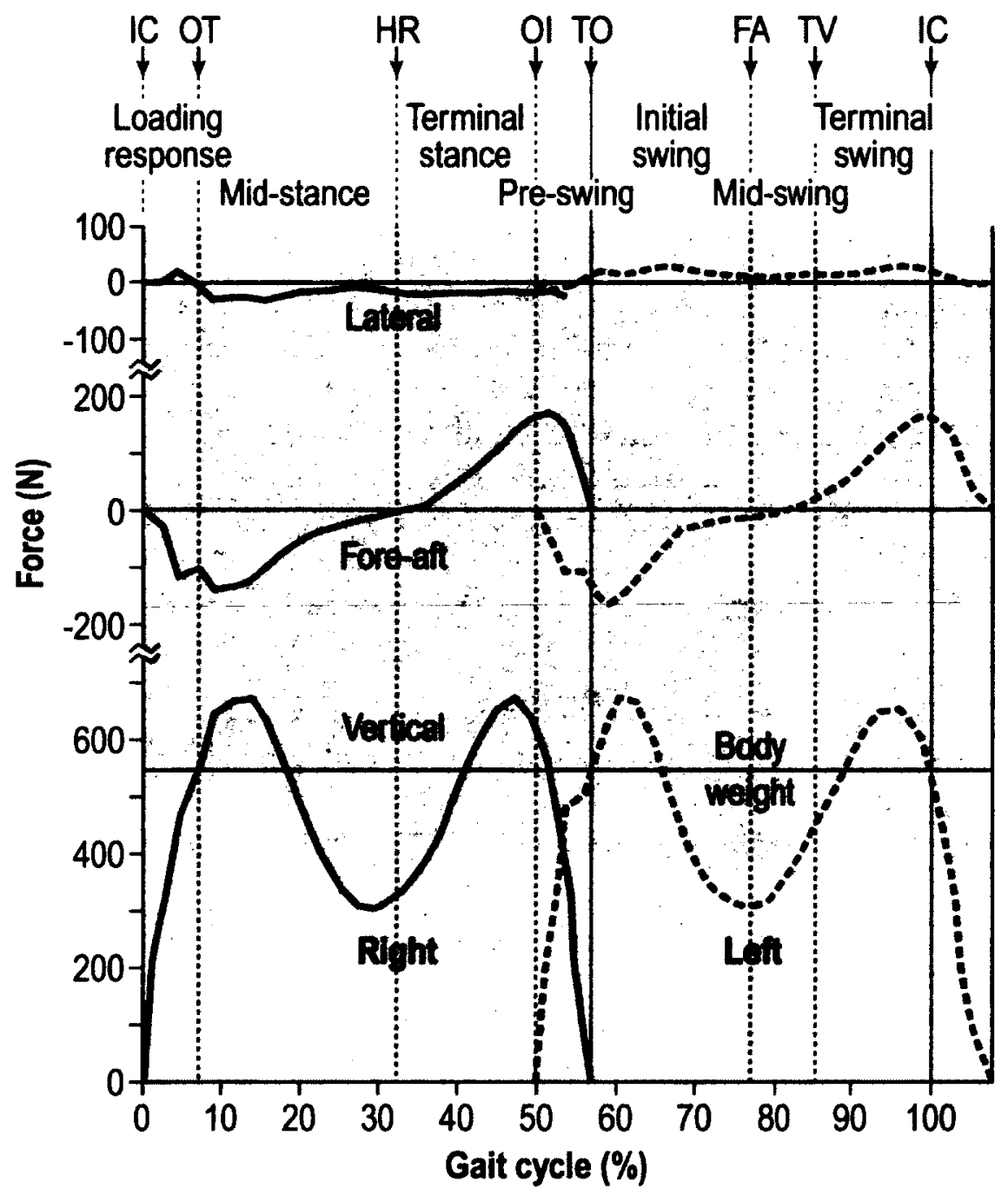

Figure 4.3: Ground reaction forces in the lateral, foreaft and vertical directions for right foot (solid line) and left foot (dashed line) [20]. 


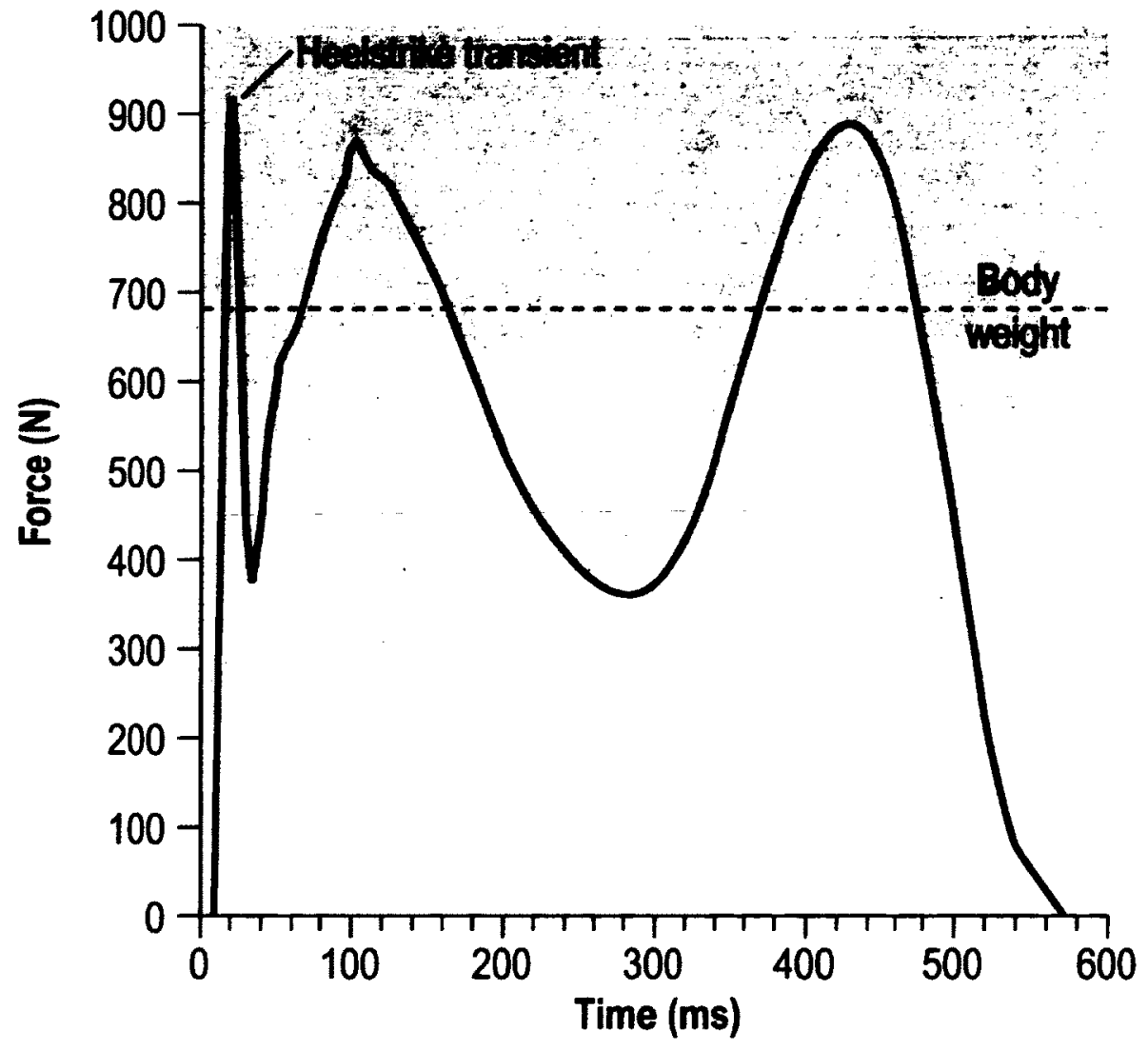

Figure 4.4: Ground reaction force vertical direction with a heelstrike transient [20]. 
appears to be applied. At initial contact, the CoP is located at the lateral side of the heel as shown in Figure 4.5. The CoP then travels along the middle of the foot until it reaches the metatarsal heads, where it moves towards the hallux at toe off.

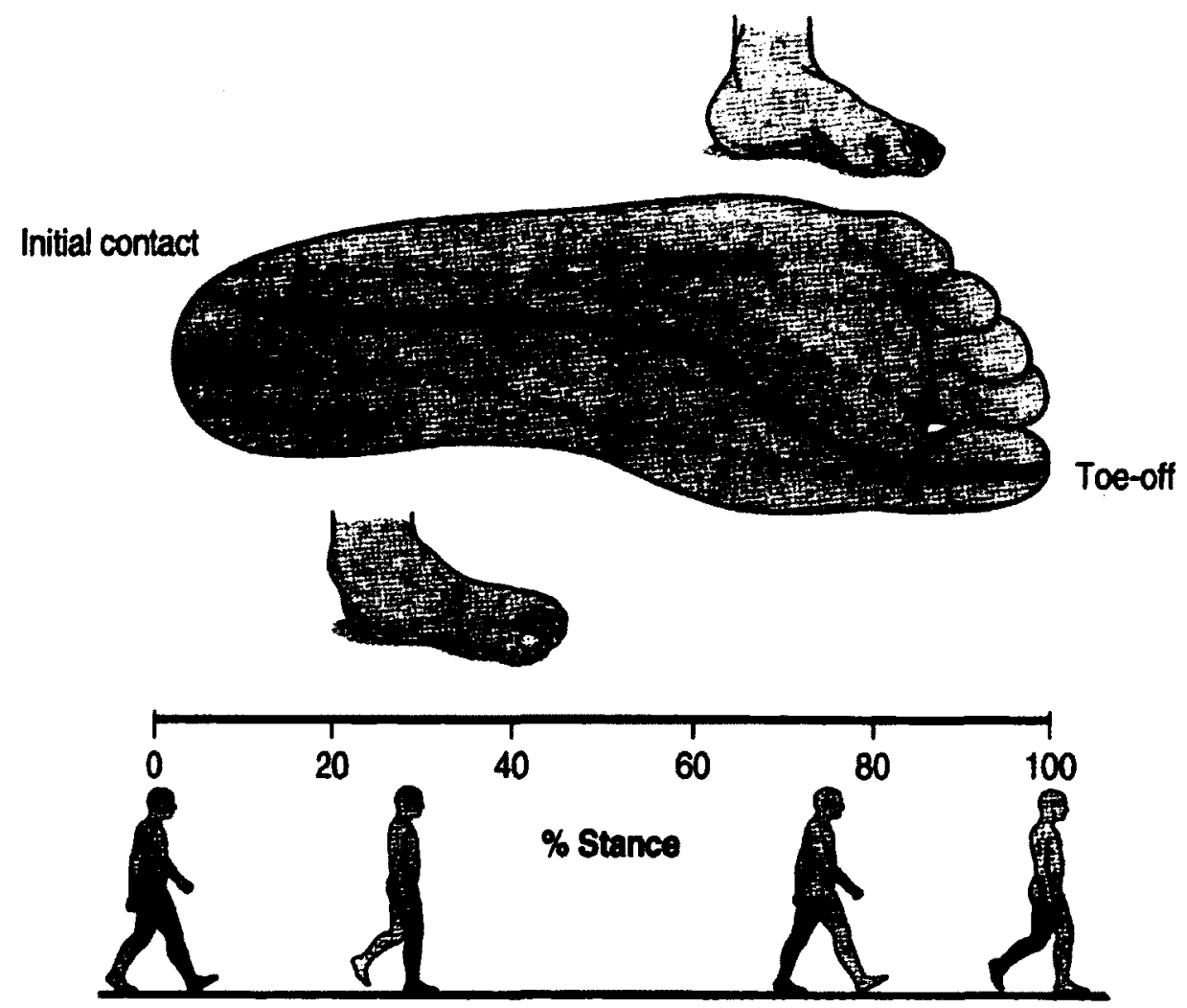

Figure 4.5: Pathway of the foot centre of pressure in normal gait [21].

\subsection{Gait Experiments}

The purpose of these experiments is to assess the ability of ViGRR to reproduce the stance and swing phase of a normal gait cycle. The control objectives related to each phase, however, are drastically different. The objective of the robot during the swing phase is to minimize the effect of end-effector dynamics on the foot by maximizing the transparency. During the stance phase, however, the control objective is to impart ground reaction forces on the foot of the user. Therefore, the gait experiments are divided into two sections in order to isolate these objectives. 


\section{Average Gait Trajectory}

During the gait experiments, a trajectory is displayed to the user as a guideline for the position of their foot during the gait cycle. This trajectory is determined based on experimental gait data acquired from healthy subjects and was obtained from [56]. Although a gait analysis clinic could be used as a more accurate means of measuring the user's gait kinematics, this would be time consuming and provide little benefit given the fact that the trajectory is used as a guideline only.

\section{Subject}

A single healthy female subject of age 22 was chosen to participate in all experiments listed below. The dimensions of this subject can be seen in Table 4.1. The gait trajectory based on these dimensions can be seen in Figure 4.6. The trajectory is oriented at an angle of $20^{\circ}$ from vertical to be consistent with the experiments below.

The purpose of the following experiments is to determine the force interaction between the robot and human foot during the stance and swing phases. Therefore, only data from a single subject was recorded as a statistical analysis of the human-robot interaction with multiple subjects is beyond the scope of this thesis.

Table 4.1: Patient Dimensions.

\begin{tabular}{c|c}
\hline Hip to Knee & $0.3810 \mathrm{~m}$ \\
Knee to Malleolus & $0.3429 \mathrm{~m}$ \\
Malleolus to Metatarsel & $0.1286 \mathrm{~m}$ \\
\hline \hline Mass & $130 \mathrm{~kg}$ \\
\hline
\end{tabular}

\subsubsection{Swing Phase Experiments}

The swing phase experiments below attempt to highlight the transparency of ViGRR during free motion. A high transparency allows the user to control the position of the robot with a small amount of force input. Ideally, the user should feel only the weight of their own leg as it swings in the air, however this is difficult to achieve with a haptic system as there are 


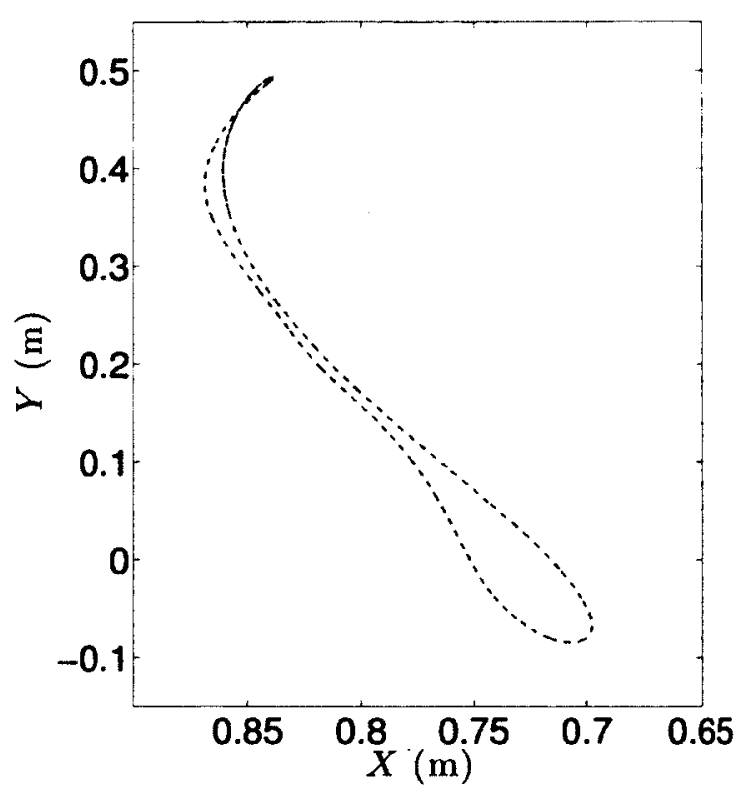

Figure 4.6: Reference gait trajectory based on patient dimensions.

physical limits to the lowest mass and damping which can be displayed.

\section{Experiment:}

The experimental setup of the swing phase experiments can be seen in Figure 4.7 below. In an effort to provide a comfortable position for the user, both the seat and the reference trajectory are rotated $20^{\circ}$. The user was asked to follow a reference gait trajectory with a

cadence of $7.5 \frac{\text { steps }}{\text { min }}$ and $12.5 \frac{\text { steps }}{\text { min }}$. A marker was overlaid on the reference trajectory to show the user the appropriate walking speed which must be replicated. This experiment was repeated 10 times for each cadence to determine an average of the interaction forces.

The impedance parameters of the robot during these experiments can be seen in Table 4.2. These impedance parameters were obtained from a preliminary experiment which required the subject to move their foot in free motion. The impedance of the end-effector was decreased incrementally until an oscillatory behavior was observed. 


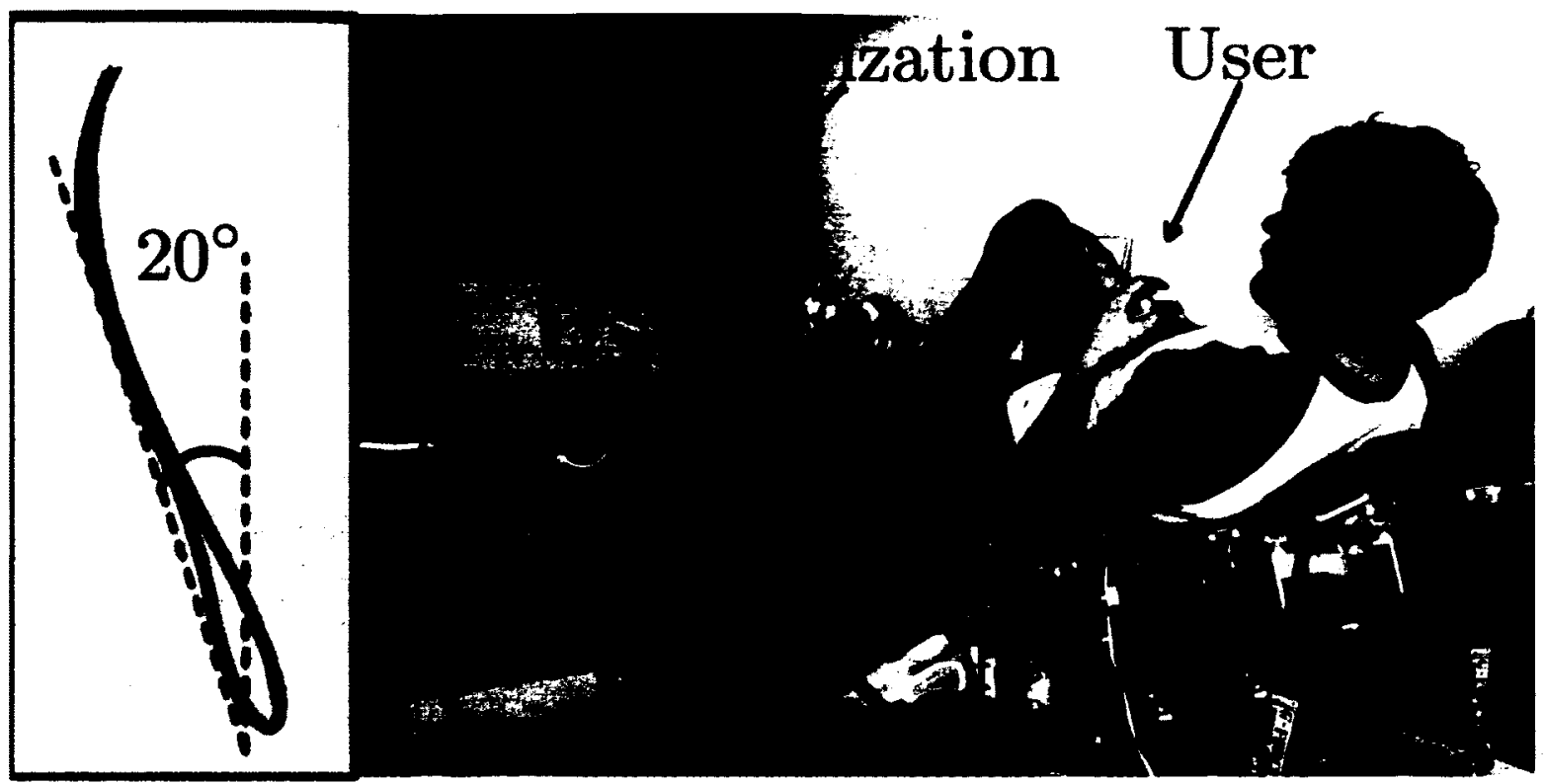

Figure 4.7: Experimental setup of the swing phase trajectory tracking experiments.

Table 4.2: Settings for the Swing Phase Experiments.

\begin{tabular}{c|c|c|c}
\hline \multirow{2}{*}{ Setting } & Parameter & Experiment 1 & Experiment 2 \\
\hline \multirow{4}{*}{ Admittance Gains } & $M_{X Y}$ & $10 \mathrm{~kg}$ & $10 \mathrm{~kg}$ \\
\cline { 2 - 4 } & $M_{\phi}$ & $1 \mathrm{kgm}$ & $1 \mathrm{kgm}^{2}$ \\
\cline { 2 - 4 } & $B_{X Y}$ & $55 \frac{\mathrm{Ns}}{\mathrm{m}}$ & $55 \frac{\mathrm{Ns}}{\mathrm{m}}$ \\
\cline { 2 - 4 } & $B_{\phi}$ & $10 \frac{\mathrm{Nms}}{\mathrm{rad}}$ & $10 \frac{\mathrm{Nms}}{\mathrm{rad}}$ \\
\hline \hline \multirow{2}{*}{ Cadence } & $C$ & $7.5 \frac{\mathrm{steps}}{\mathrm{min}}$ & $12.5 \frac{\mathrm{steps}}{\mathrm{min}}$ \\
\hline
\end{tabular}




\section{Results:}

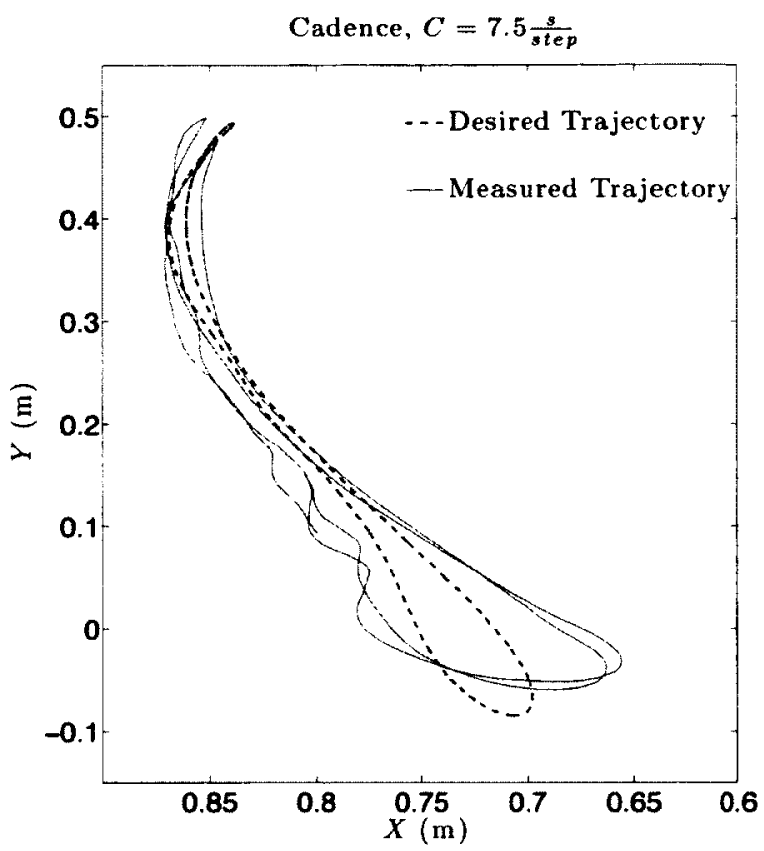

(a)

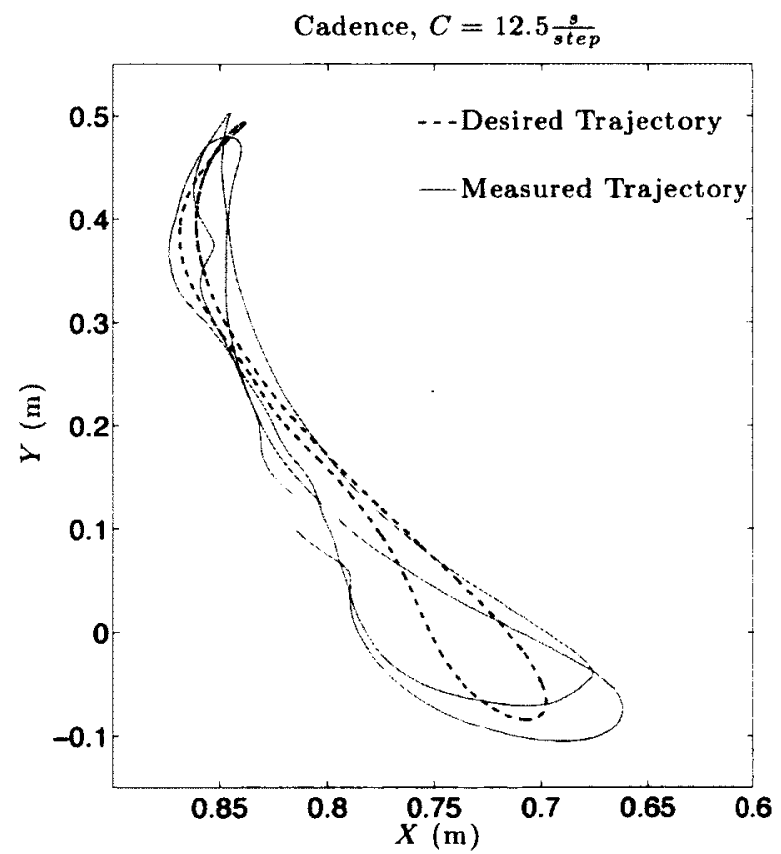

(b)

Figure 4.8: Desired and measured gait trajectories during two swing phase trials for a cadence of (a) $7.5 \frac{\text { steps }}{\mathrm{min}}$ and (b) $12.5 \frac{\text { steps }}{\mathrm{min}}$. 

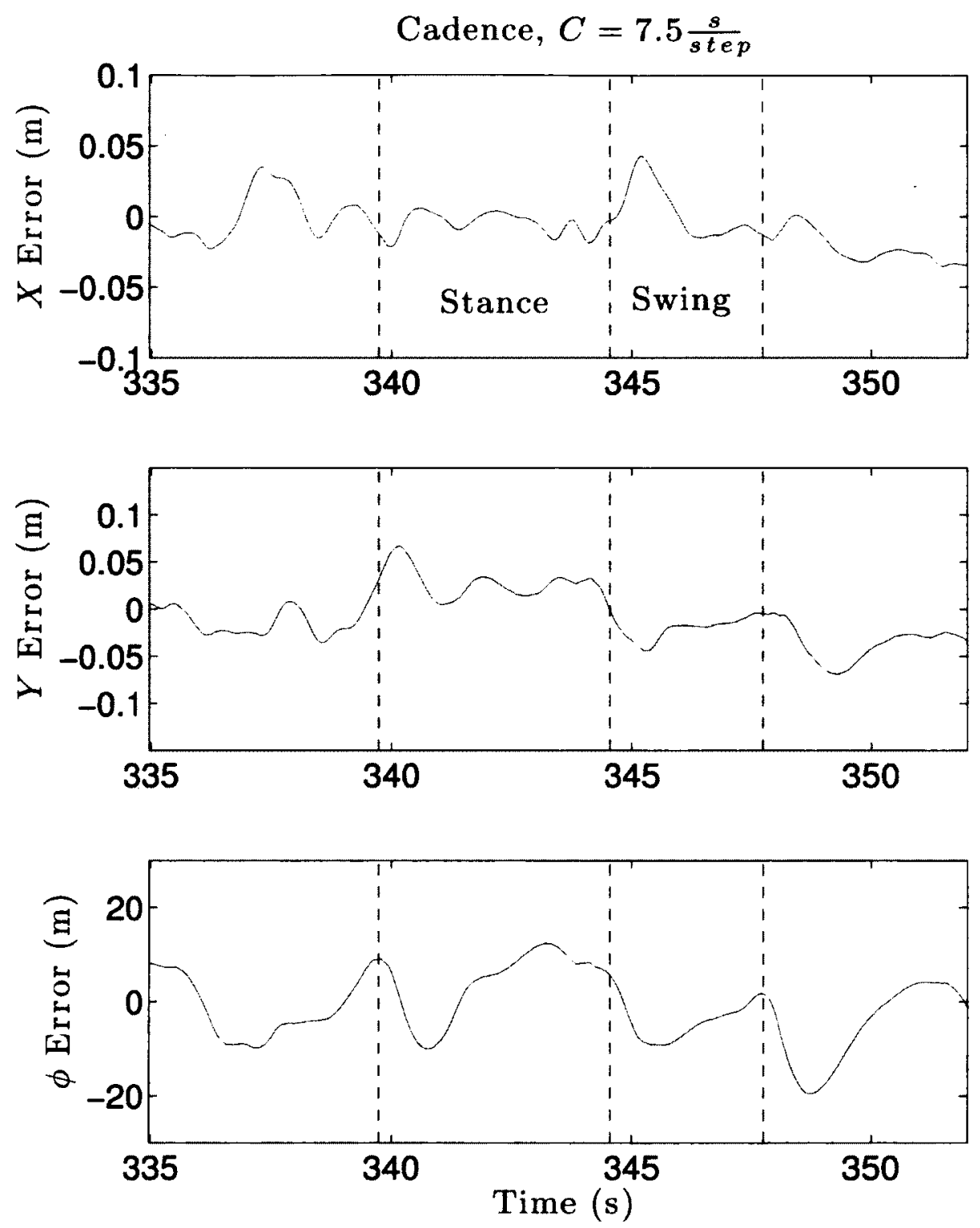

Figure 4.9: Position errors during one trial of the swing phase trajectory experiment for a cadence, $C=7.5 \frac{\text { steps }}{\text { min }}$. 

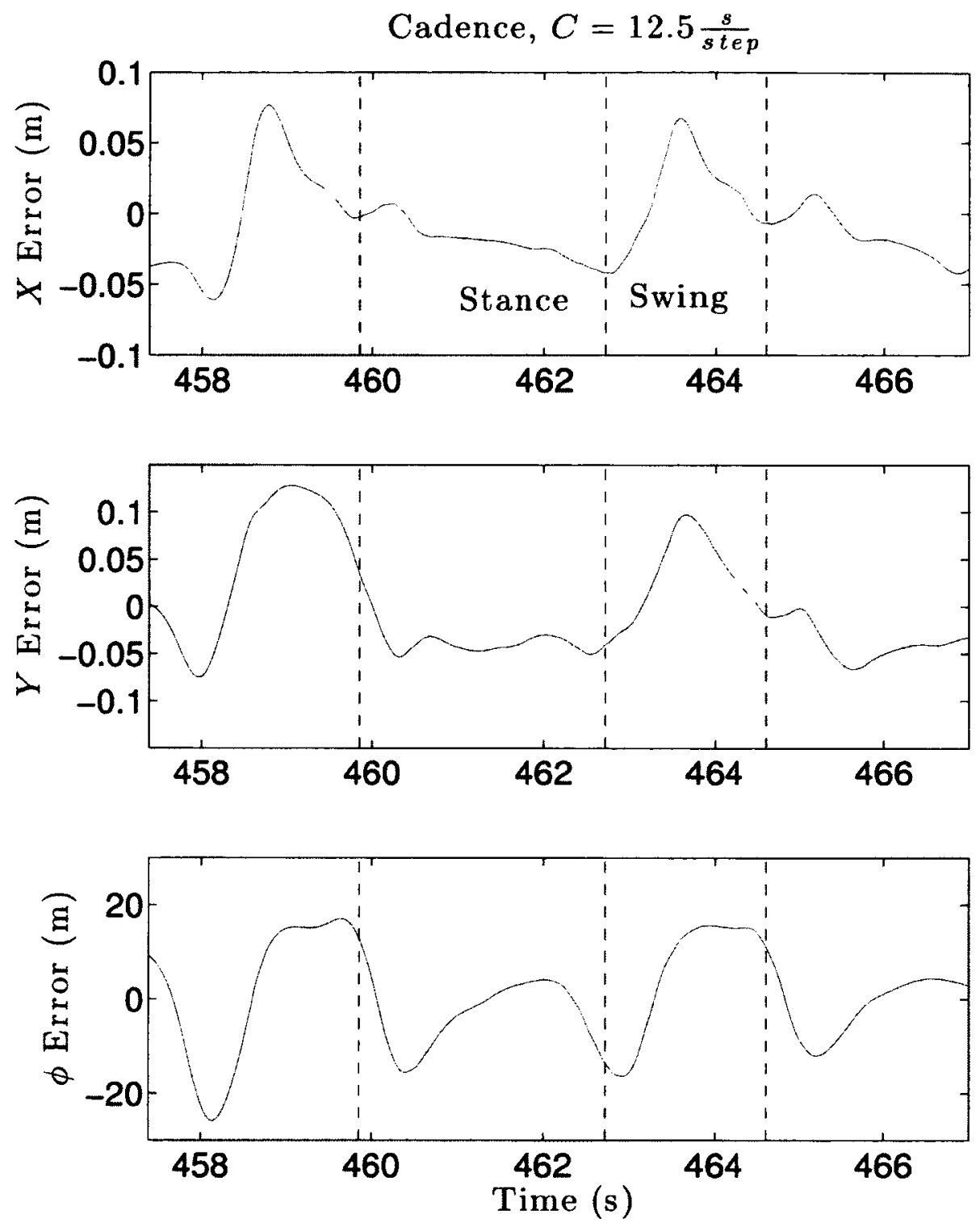

Figure 4.10: Position errors during one trial of the swing phase trajectory experiment for a cadence, $C=12.5 \frac{\text { steps }}{\text { min }}$. 

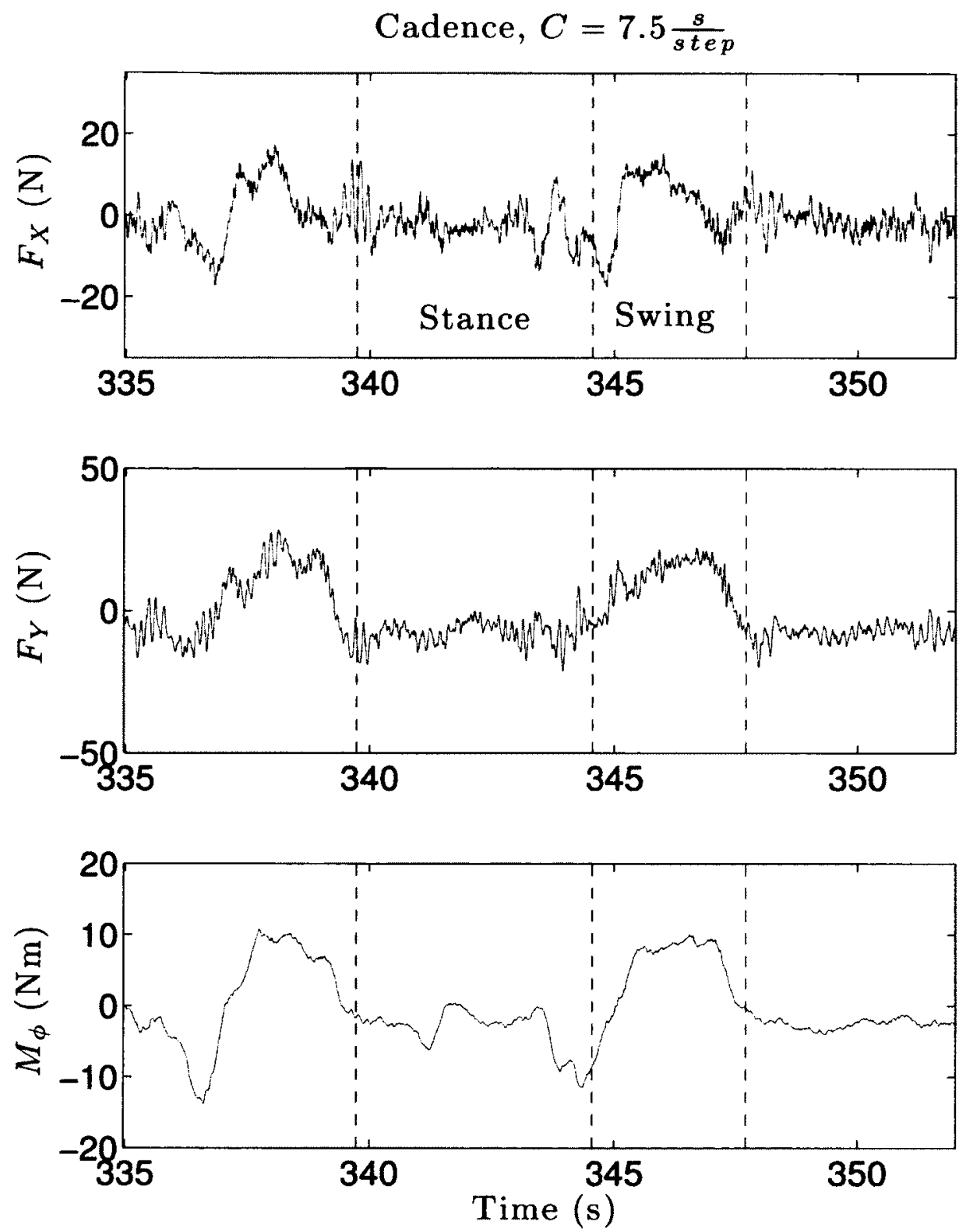

Figure 4.11: Interaction loads during one trial of the swing phase trajectory experiment for a cadence, $C=7.5 \frac{\text { steps }}{\min } . F_{x}, F_{y}$, and $M_{\phi}$ represent the forces in the $X$ and $Y$ directions and the moment about the $\phi$ axis respectively. 

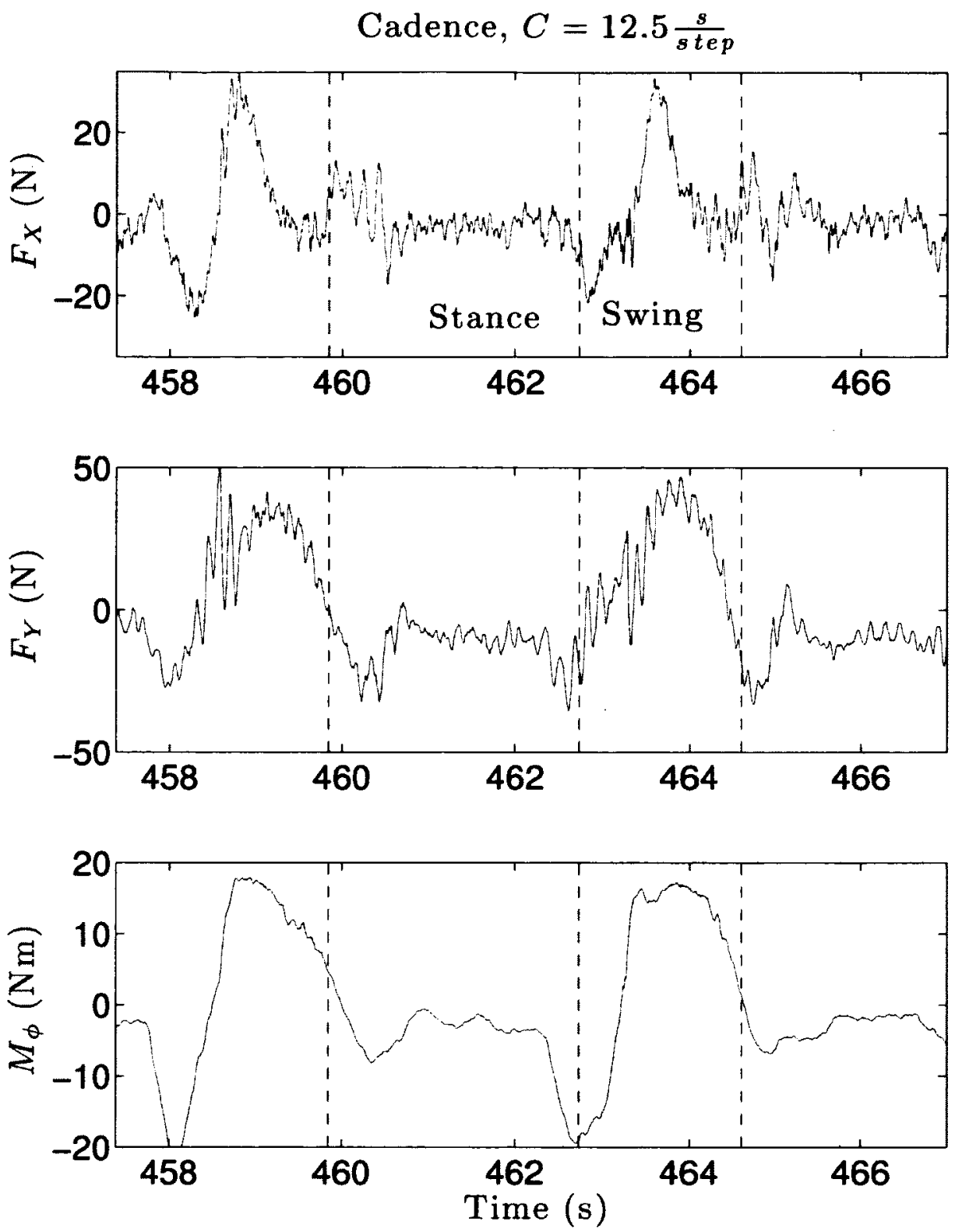

Figure 4.12: Interaction loads during one trial of the swing phase trajectory experiment for a cadence, $C=7.5 \frac{\text { steps }}{\min } . F_{x}, F_{y}$, and $M_{\phi}$ represent the forces in the $X$ and $Y$ directions and the moment about the $\phi$ axis respectively.

\section{Quantitative Discussion:}

\section{Trajectory Tracking and Errors}

The trajectory tracking plots of positions and errors with respect to time can be seen 
in Figures 4.8, 4.9, and 4.10. A general trend that can be seen in Figure 4.9 for a cadence of $7.5 \frac{\text { steps }}{\mathrm{min}}$ is an increase in tracking error during the swing phase of the gait cycle. This increase in error is due to the fact that the subject must fight against gravity to propel their leg through the swing phase. As the cadence increases, the speed of the foot must also increase to track the trajectory closely. However, the impedance of the device acts to hinder the motion of the leg and causes the tracking error to increase. This error becomes more evident as the cadence is increased to $12.5 \frac{\text { steps }}{\mathrm{min}}$ as seen in Figure 4.10. Maximum tracking errors for both experiments are shown in Table 4.3 below.

Table 4.3: Maximum Tracking Errors for a Gait Trajectory.

\begin{tabular}{c|c|c}
\hline Direction & Cadence $=7.5 \frac{\text { steps }}{\min }$ & Cadence $=12.5 \frac{\text { steps }}{\min }$ \\
\hline \hline$X$ & $4.3 \mathrm{~cm}$ & $7.7 \mathrm{~cm}$ \\
$Y$ & $6.7 \mathrm{~cm}$ & $12.8 \mathrm{~cm}$ \\
$\phi$ & $12.4^{\circ}$ & $17.1^{\circ}$ \\
\hline
\end{tabular}

\section{Forces and Moments}

The swing phase experiments were conducted through 10 gait cycles. The plots of userrobot interaction forces for a single gait cycle can be seen in Figures 4.11 and 4.12 for a cadence of 7.5 and $12.5 \frac{\text { steps }}{\mathrm{min}}$ respectively. The peak force values of both the swing and stance phases were averaged over 10 gait cycles using the findpeaks function in Matlab. The forces in $X$ and $Y$ during the stance phase reach average peak values of $15 \mathrm{~N}$ and $20 \mathrm{~N}$ respectively. Once the swing phase begins, the forces in $X$ and $Y$ increase to average maximum values of $17 \mathrm{~N}$ and $28 \mathrm{~N}$ in order to decelerate the foot and accelerate it against gravity. Also, the moment about $\phi$ at the beginning of the swing phase changes directions to rotate the foot from toe off to heel down. Once again, these trends become more pronounced as the speed of the gait is increased as seen in 4.12 since the device requires more force to move at a higher velocity.

As a comparison of the transparency of ViGRR with respect to the HapticWalker, Figure 4.13 shows the interaction forces during a free motion experiment for the HapticWalker. The subject was asked to stand on a virtual floor with their left foot and move their right foot 
in the air. The interaction loads between the HapticWalker and user reach maximums of $80 \mathrm{~N}$ in $X, 60 \mathrm{~N}$ in $Y$, and $15 \mathrm{Nm}$ in $\phi$. Although these motions are not based on a gait cycle, they do show that the user must apply more force to move the end-effector of the HapticWalker than is necessary for ViGRR. The maximum loads achieved from the swing phase experiments can be seen in Table 4.4 below.
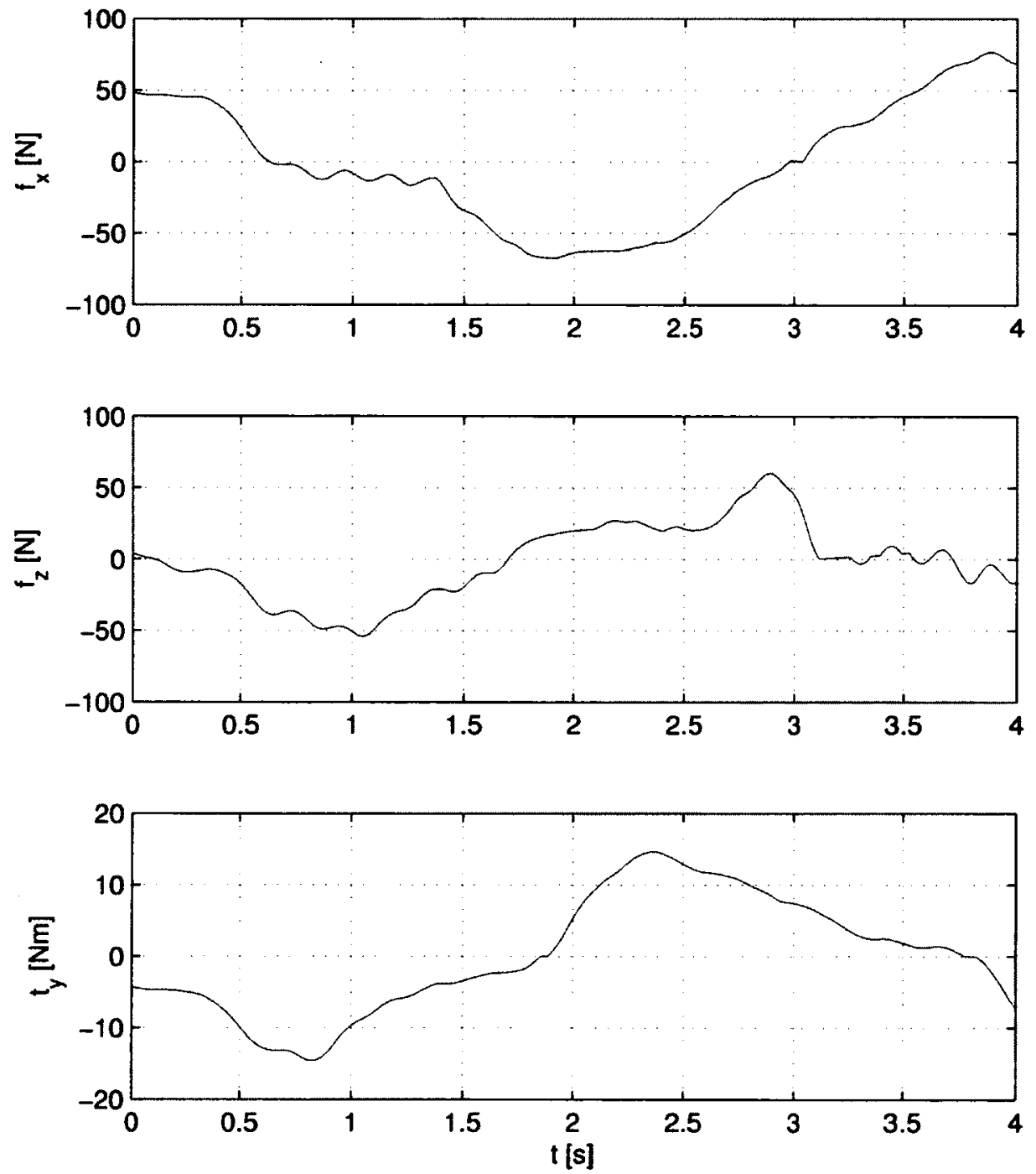

Figure 4.13: Force and torque measurements during free motion experiments for the HapticWalker [10]. 
Table 4.4: Maximum Interaction Loads for a Gait Trajectory.

\begin{tabular}{c|c|c|c}
\hline Load & Cadence $=7.5 \frac{\text { steps }}{\text { min }}$ & Cadence $=12.5 \frac{\text { steps }}{\text { min }}$ & HapticWalker \\
\hline \hline$F_{X}$ & $17.3 \mathrm{~N}$ & $34.6 \mathrm{~N}$ & $80 \mathrm{~N}$ \\
$F_{Y}$ & $28.3 \mathrm{~N}$ & $52.0 \mathrm{~N}$ & $60 \mathrm{~N}$ \\
$M_{\phi}$ & $10.8 \mathrm{Nm}$ & $17.9 \mathrm{Nm}$ & $15 \mathrm{Nm}$ \\
\hline
\end{tabular}

\section{Qualitative Discussion:}

\section{Subject Feedback}

After the experiments were completed, the subject was asked to describe the fidelity of the swing phase reproduction. Overall, the subject found that the impedance parameters chosen for both experiments were too large to allow for a smooth trajectory tracking. During

the first experiment ( $C=7.5 \frac{\text { steps }}{\mathrm{min}}$ ), the subject felt that she was able to track the trajectory until the end of the stance phase. At this point the effect of gravity and the relatively large inertia $\left(M_{\phi}=1 \mathrm{kgm}^{2}\right)$ prevented the subject from completing the motion. This large tracking error can be seen at the bottom of both plots in Figure 4.8. Although the tracking errors were increased with a faster walking speed, the subject felt that the interaction with the device became smoother.

At the completion of both experiments the subject felt signs of leg fatigue due to the interaction loads and lack of gravity compensation.

\section{Foot vs. Hand Coupling}

As can be seen by comparing Tables 4.2 and 3.1, the admittance parameters that allowed for a stable, patient-robot coupling are higher for the foot than for the hand. There are several possible reasons behind this.

First, the hand is coupled to the end-effector of ViGRR by grasping the footplate whereas the foot is coupled to the footplate through two velcro straps as seen in Figure 4.14. The loose coupling of the hand acts as a filter to mitigate oscillations whereas the foot seems to have a more rigid contact with the footplate. 


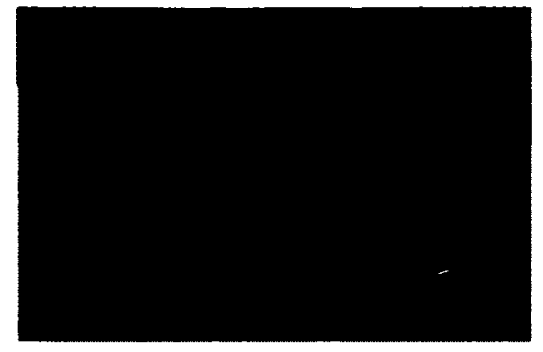

(a) Hand Coupling

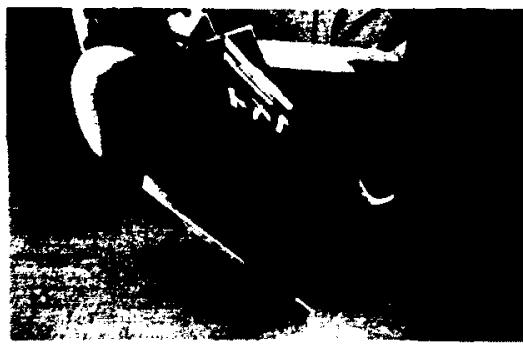

(b) Foot Coupling

Figure 4.14: Device-Body Coupling of ViGRR for the hand and foot.

Another possible reason which can be attributed to higher admittance parameters for foot coupling is that the foot is controlled by larger muscle groups than the hand. The arm is ideal for accurate tasks where fine motor control is needed whereas the leg has larger muscles which are ideal for power applications. Since the human leg is not able to provide accurate forces, the robot must be adapted to fit the characteristics of the human leg.

It is possible that all of these factors contribute to the behavior of the human-robot interface, however more biomedical experiments are required in order to determine more concrete results.

\section{Gravity Compensation}

During the experiments, the user was exerting effort by moving the end-effector through the gait trajectory, while simultaneously fighting gravity to keep the leg in the air. This added strain may cause fatigue on the leg muscles during extended periods of use, and could also activate larger muscle groups which are deemed more suitable for gross motor control. Ideally a feed-forward gravity compensation term should be added to the admittance controller which would cancel gravitational loads produced at the end-effector as seen in Figure 4.15. However, this gravity compensation term would require a measurement of the user's joint angles in real-time, along with an accurate model of the leg parameters of the user. These leg parameters are difficult to achieve in practice and are not within the scope of this thesis. 


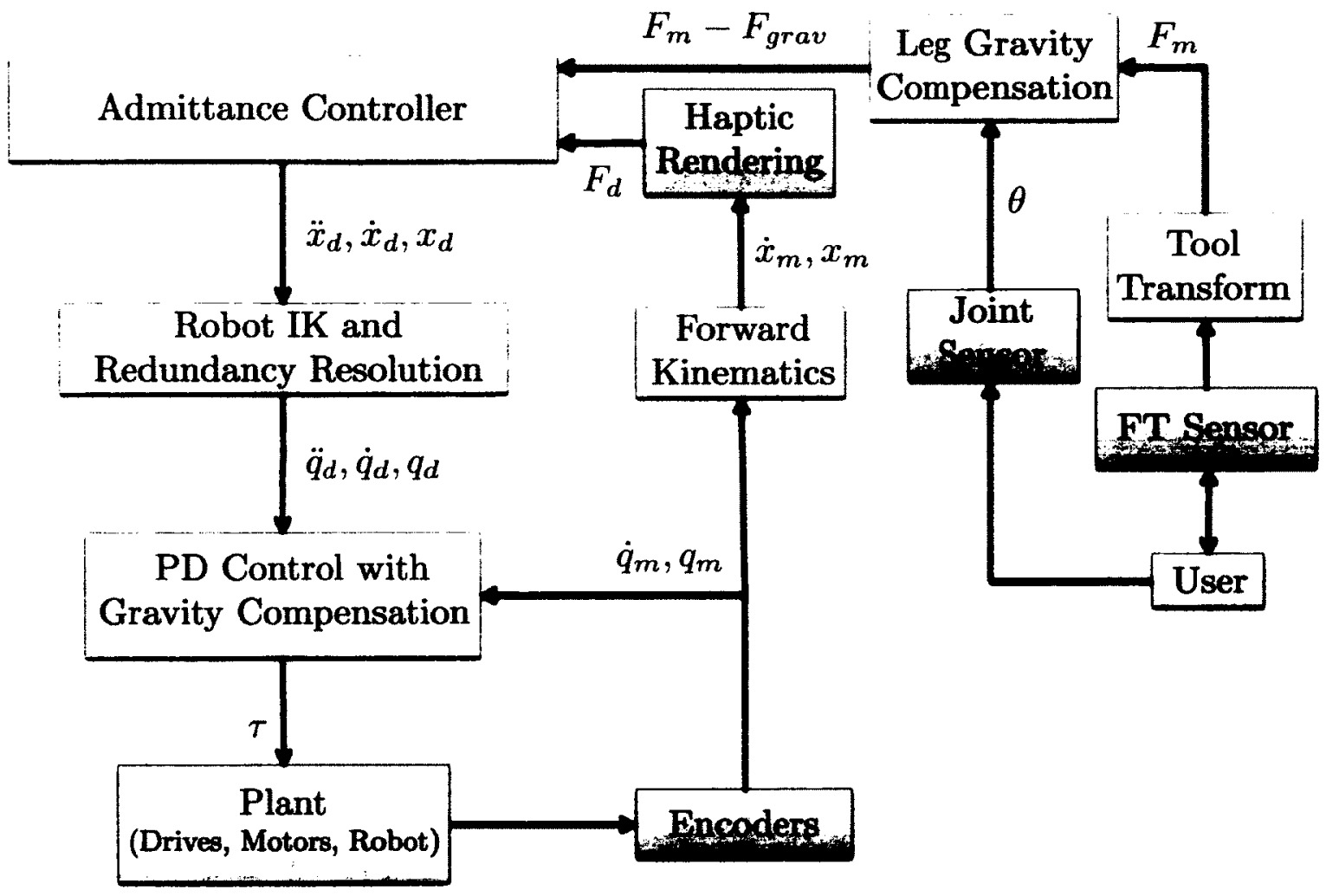

Figure 4.15: Gravity compensation admittance controller.

A simple leg gravity compensation scheme is proposed which approximates the leg parameters of the user as a two bar linkage. The first link spans from the hip joint of the user to the knee, whereas the second link spans from the knee to the end-effector as seen in Figure 4.16 below. The gravity compensation force, $F_{\text {grav }}$ shown above can then be calculated through

$$
F_{g r a v}=J_{g r a v}(\theta)^{-T} G_{g r a v}(\theta)
$$

where

$$
J_{g r a v}(\theta)=\left[\begin{array}{cc}
-l_{g 1} \sin \left(\theta_{1}\right)-l_{g 2} \sin \left(\theta_{1}+\theta_{2}\right) & -l_{g 2} \sin \left(\theta_{1}+\theta_{2}\right) \\
l_{g 1} \cos \left(\theta_{1}\right)+l_{g 2} \cos \left(\theta_{1}+\theta_{2}\right) & l_{g 2} \cos \left(\theta_{1}+\theta_{2}\right)
\end{array}\right]
$$


and

$$
G_{\text {grav }}(\theta)=\left[\begin{array}{c}
m_{g 1} g l_{c g 1} \cos \left(\theta_{1}\right)+m_{g 2} g\left(l_{g 1} \cos \left(\theta_{1}\right)+l_{g 2} \cos \left(\theta_{1}+\theta_{2}\right)\right) \\
m_{g 2} g l_{c g 2} \cos \left(\theta_{1}+\theta_{2}\right)
\end{array}\right]
$$

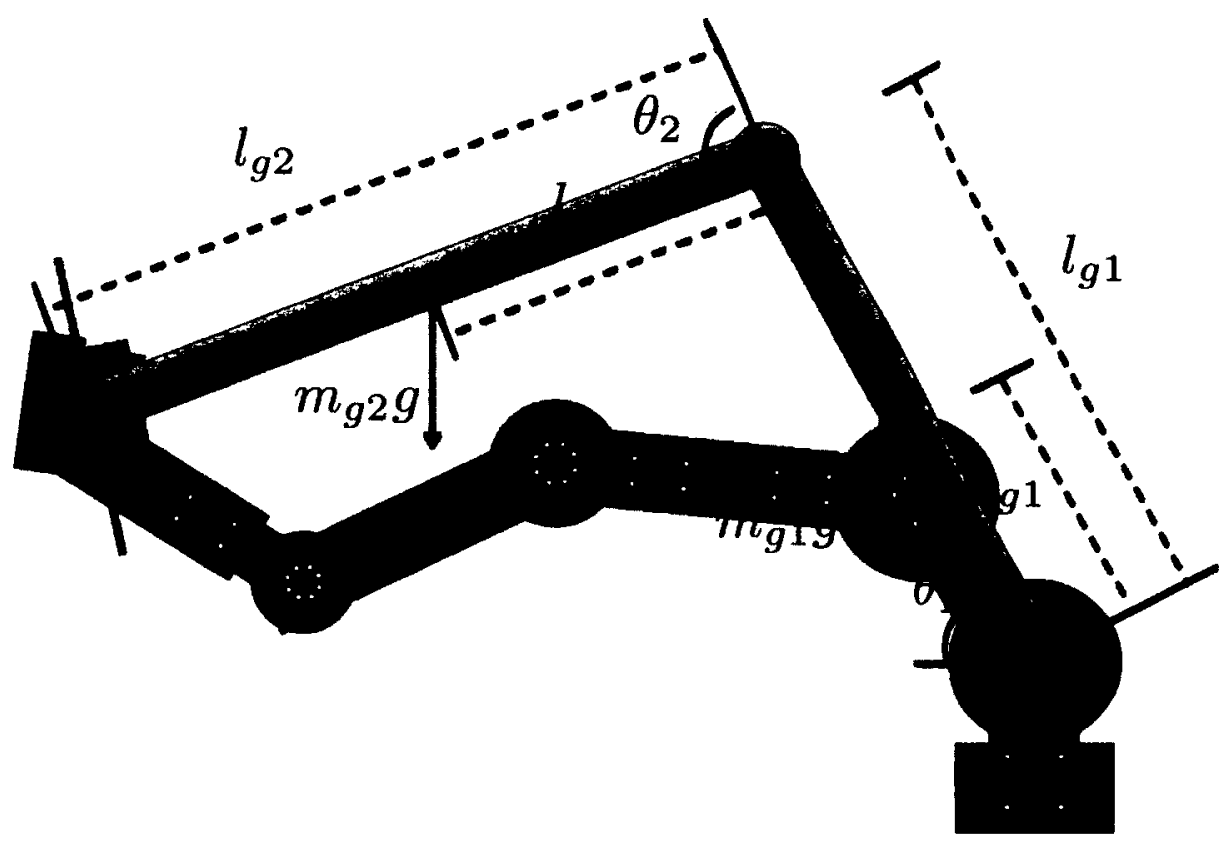

Figure 4.16: Simple gravity compensation.

\section{Discussion:}

Results from the previous section show that although the subject was able to track the reference trajectory through most of the gait cycle, the admittance parameters associated with the end-effector control are too high to allow for a smooth, unhindered swing phase. The admittance parameters, however cannot be decreased without incurring instability due to biomedical feedback from the user's foot. A more in depth study into the cause of the instability is recommended in order to decrease the admittance parameters and allow for a more transparent human-robot interaction. Also, in order to increase the fidelity of the walking simulation and decrease subject fatigue, a gravity compensation method must be 
introduced such as the one described above.

\subsubsection{Stance Phase Experiments}

These experiments are directed towards determining the ability of ViGRR to reproduce a scaled version of the vertical ground reaction force normally seen through walking. As seen in Figure 4.3, peaks in the vertical ground reaction force should reach around $110 \%$ of the user's body weight. ViGRR, however was designed to operate at roughly $60 \%$ of the mass of a healthy male subject. Therefore, it is clear that the ground reaction forces produced with these experiments will not reach the loads seen during normal walking. The intention of these experiments, however is not to fully replicate the exact GRF profile but to determine the effect of several virtual tool geometries on the shape of the vertical GRF. These virtual tools will also be evaluated on how accurately the centre of pressure of the user's foot is tracked during the stance phase.

\section{Virtual Tool Profiles}

As demonstrated in the previous chapter, it is shown that the forces produced by the haptic algorithm are dependant on the shape of the virtual tool. The question that needs to be answered now is: What virtual tool geometry is optimal at generating ground reaction forces for the foot? Three virtual tool geometries, as seen in Figure 4.17 were tested with the haptic rendering algorithm in order to determine the best possible solution. A rectangular geometry was first used as the virtual tool to generate ground reaction forces. This profile has dimensions of $0.3 m \times 0.01 m$ and is oriented along the axis of the footplate. The second virtual tool tested is a circular geometry with a radius of $0.3 m$. Finally, a hybrid geometry is used for the virtual tool which has dimensions of $0.3 m \times 0.05 m$ and elliptical curves at the toe and heel. It is proposed that the curved surfaces will provide a smoother interaction with the virtual ground during the stance phase. 

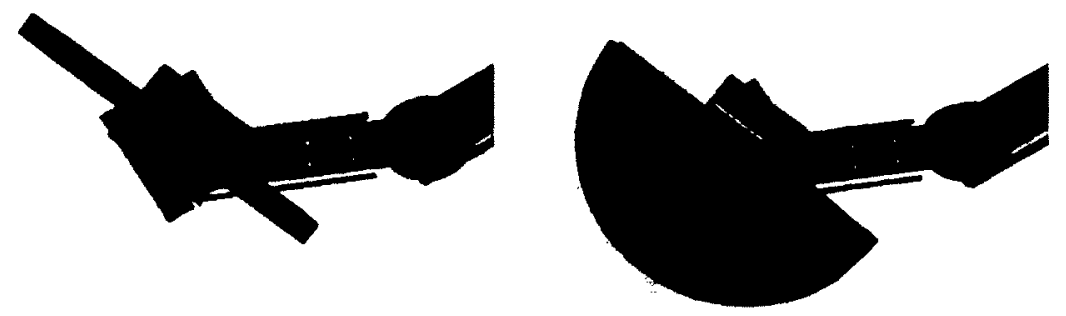

(a)

(b)

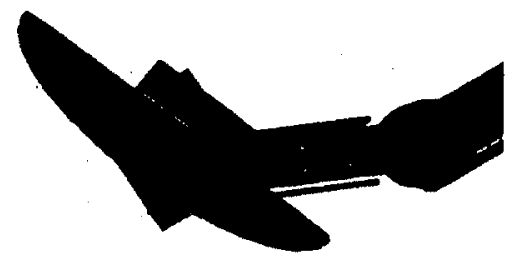

(c)

Figure 4.17: Virtual tool profiles for producing ground reaction forces (a) Rectangular (b) Cirular (c) Hybrid.

\section{Experiment:}

The stance phase experiment has a similar setup to the swing phase experiment, with the user inclined at $20^{\circ}$ from the horizontal. The human subject is instructed to follow the same gait trajectory as mentioned above, however a virtual ground is aligned with the trajectory in the vertical direction ${ }^{1}$ to produce contact forces as seen in Figure 4.18. These experiments are conducted without friction in order to isolate the influence of the normal force. Table 4.5 outlines the settings of both the admittance controller and haptics for all three virtual tool experiments. The haptic parameters $K$ and $D$ were experimentally determined for stable contact with the virtual ground and are specific to the subject conducting the experiments.

\footnotetext{
${ }^{1}$ Experiments were initially conducted with the gait trajectory rotated $20^{\circ}$ from vertical as in the swing phase experiments, however oscillations were observed whose root cause could not be determined. A preliminary analysis of these oscillations is presented in Appendix $\mathrm{C}$ in order to isolate this problem from the thesis results.
} 
If subsequent tests are to be conducted, these parameters must be determined on a subject by subject basis.

In a typical gait analysis lab, the CoP during the stance phase is calculated using $3 \mathrm{D}$ force analysis of the load applied to the forceplate. In the case of ViGRR, the stance phase is constrained to the $X Y$ plane and therefore the center of pressure calculation is simply $M_{\phi} / F_{N}$

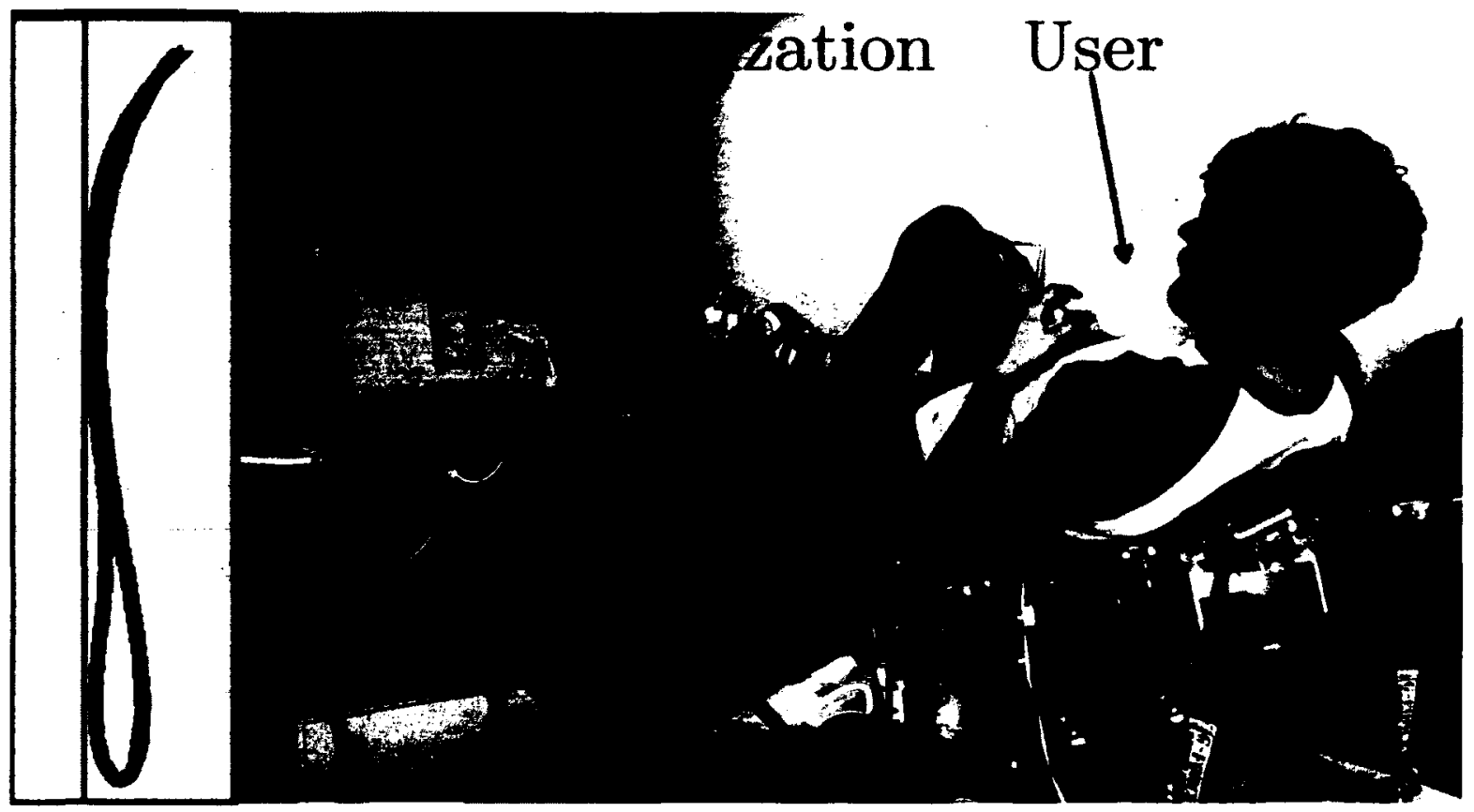

Figure 4.18: Experimental setup of the stance phase trajectory tracking experiments. 
Table 4.5: Settings for the Swing Phase Experiments.

\begin{tabular}{c|c|c}
\hline Setting & Parameter & Gain \\
\hline \hline \multirow{2}{*}{ Admittance Gains } & $M_{X Y}$ & $10 \mathrm{~kg}$ \\
& $M_{\phi}$ & $1 \mathrm{kgm}^{2}$ \\
& $B_{\phi}$ & $10 \frac{\mathrm{Nms}}{\mathrm{rad}}$ \\
\hline \hline Haptic Gains & $K$ & $2000 \frac{\mathrm{N}}{\mathrm{m}}$ \\
& $D$ & $100 \frac{\mathrm{Ns}}{\mathrm{m}}$ \\
\hline
\end{tabular}

\section{Rectangular Virtual Tool}

Results:

Figure 4.19 shows the ground reaction forces and CoP for the rectangular virtual tool. 

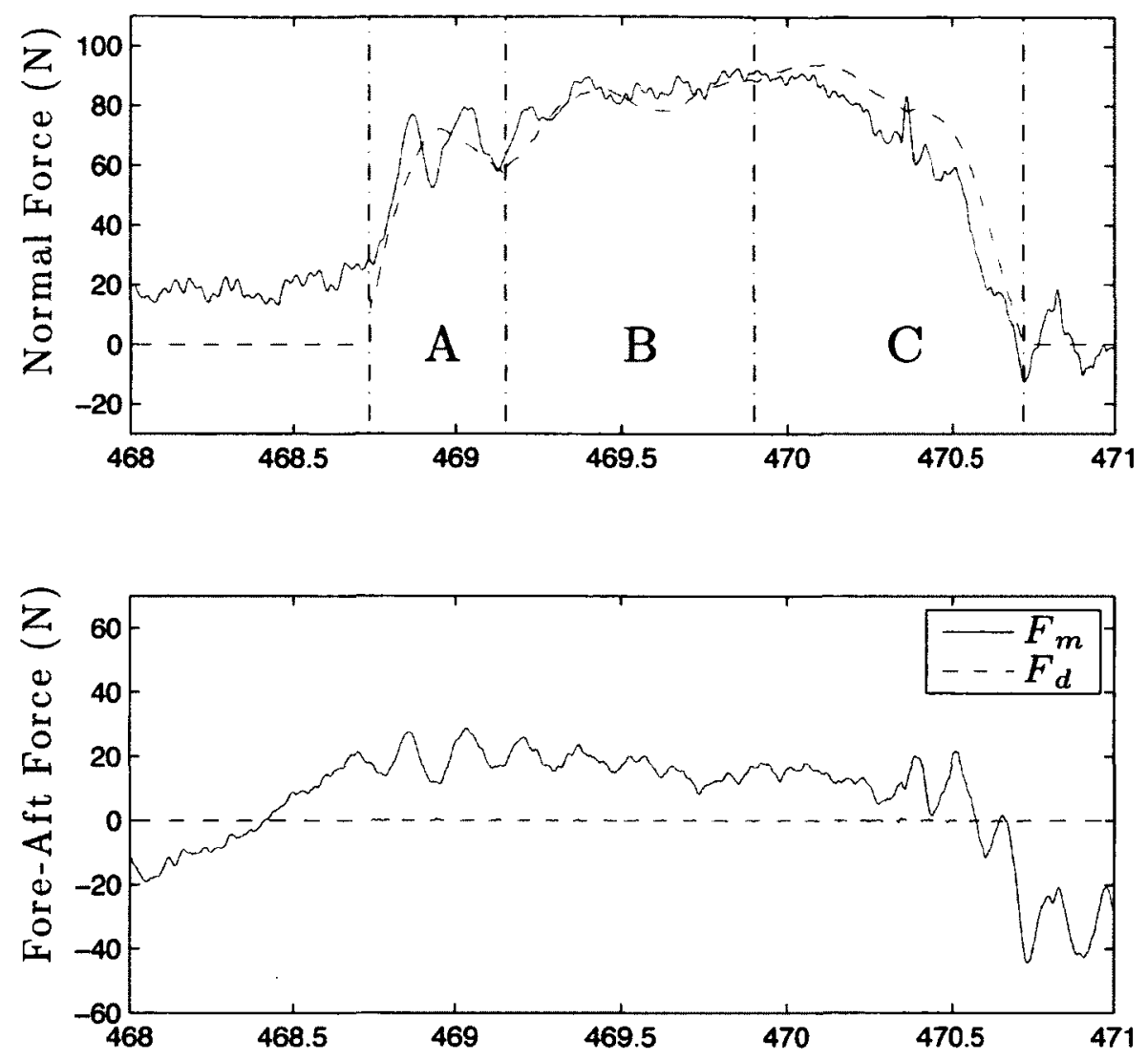

\section{Tool}

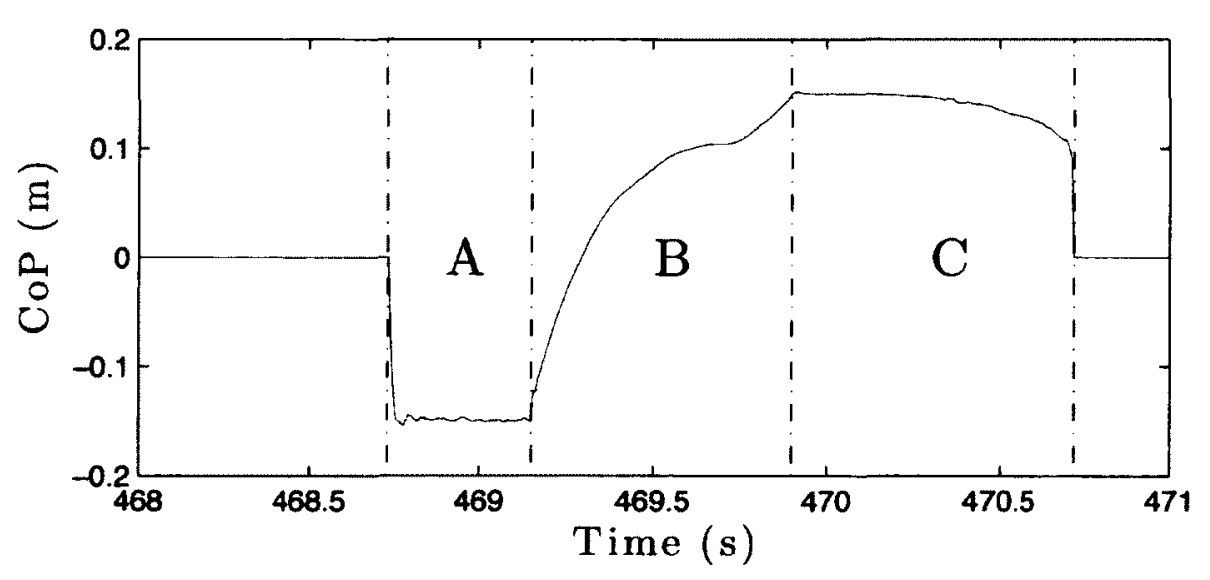
Shape

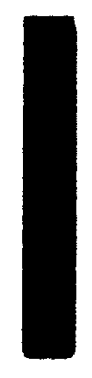

Figure 4.19: Ground Reaction Forces and CoP for a rectangular virtual tool. Sections A, $\mathrm{B}$, and $\mathrm{C}$ represent heel down, mid-stance, and toe off respectively. $F_{m}$ and $F_{d}$ represent the measured forces at the foot plate and desired forces from the haptic rendering algorithm. 


\section{Discussion:}

Normal Force: The rectangular virtual tool provides a simple geometry for generating ground reaction forces to the user's foot. As can be seen from Figure 4.19, heel down and toe off occur during sections $\mathrm{A}$ and $\mathrm{C}$ respectively. Instead of following a characteristic double hump as seen in Figure 4.3, the normal forces for the rectangular tool can be broken down into 3 sections. The first section, labelled A on Figure 4.19, is caused by the corner of the virtual tool contacting the virtual ground. Section B shows a second hump which is represented due to the flat bottom of the virtual tool contacting the ground. Finally, there is a final hump in section $\mathrm{C}$ which is caused by the toe off of the user's gait pattern.

Centre of Pressure: During section A, the CoP was constant at the corner of the virtual tool which gives the sensation of a hinge joint at the heel of the user's foot. The CoP should be moving along the cross-section of the foot during a normal gait cycle. Once the virtual tool has made contact with the ground, the CoP travels along the cross section of the virtual tool which is consistent with gait data.

Subject Feedback: The rectangular virtual tool provides an unnatural feeling for the user as the dicrete corners and flat geometry of the virtual tool prevent the foot from moving smoothly across the virtual ground. When asked about the fidelity of the walking simulation, the user felt as if the bottom of their foot was tied to a block.

\section{Circular Virtual Tool}

\section{Results:}

Figure 4.20 shows the ground reaction forces and $\mathrm{CoP}$ for the circular virtual tool. 

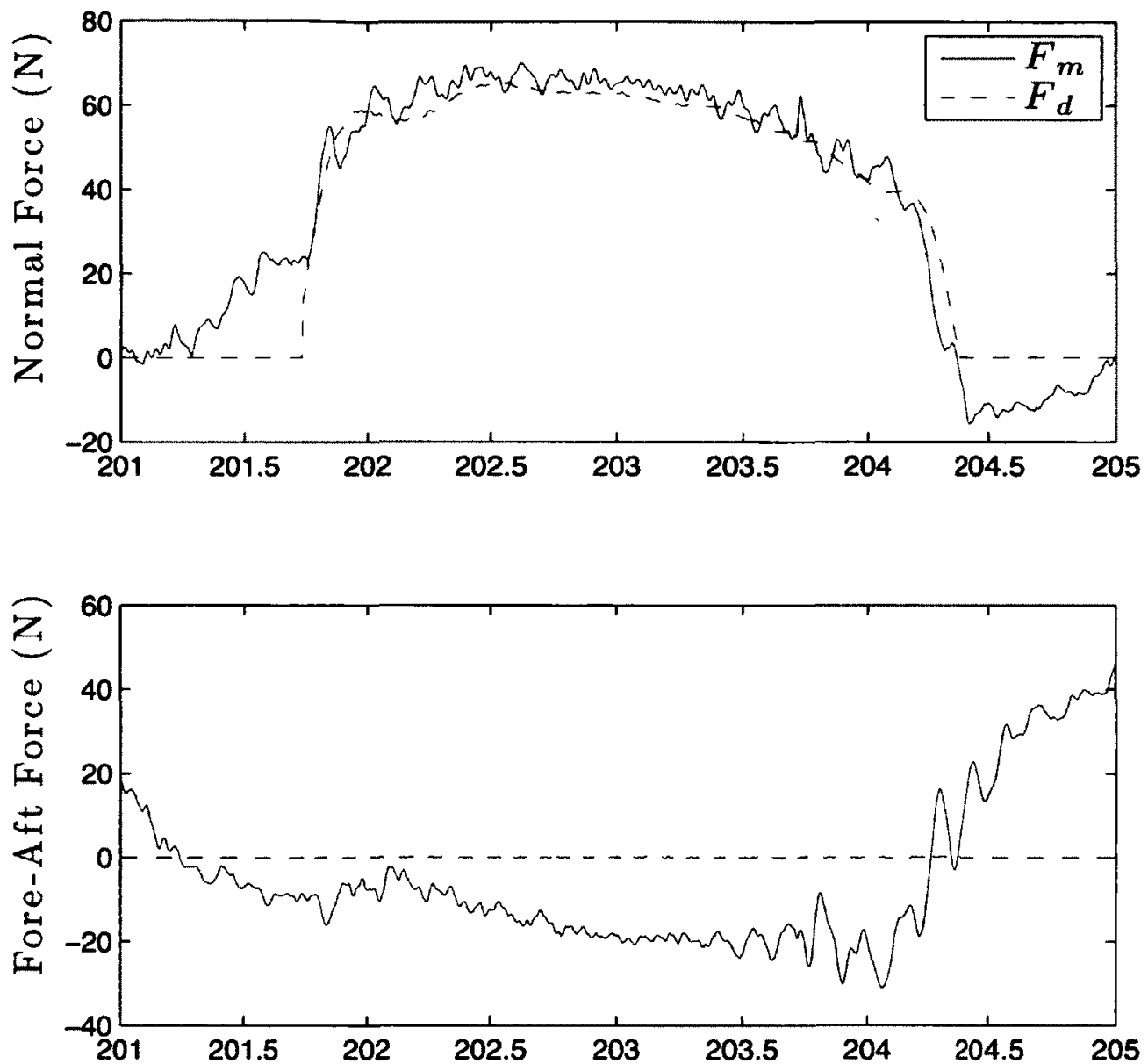

Tool

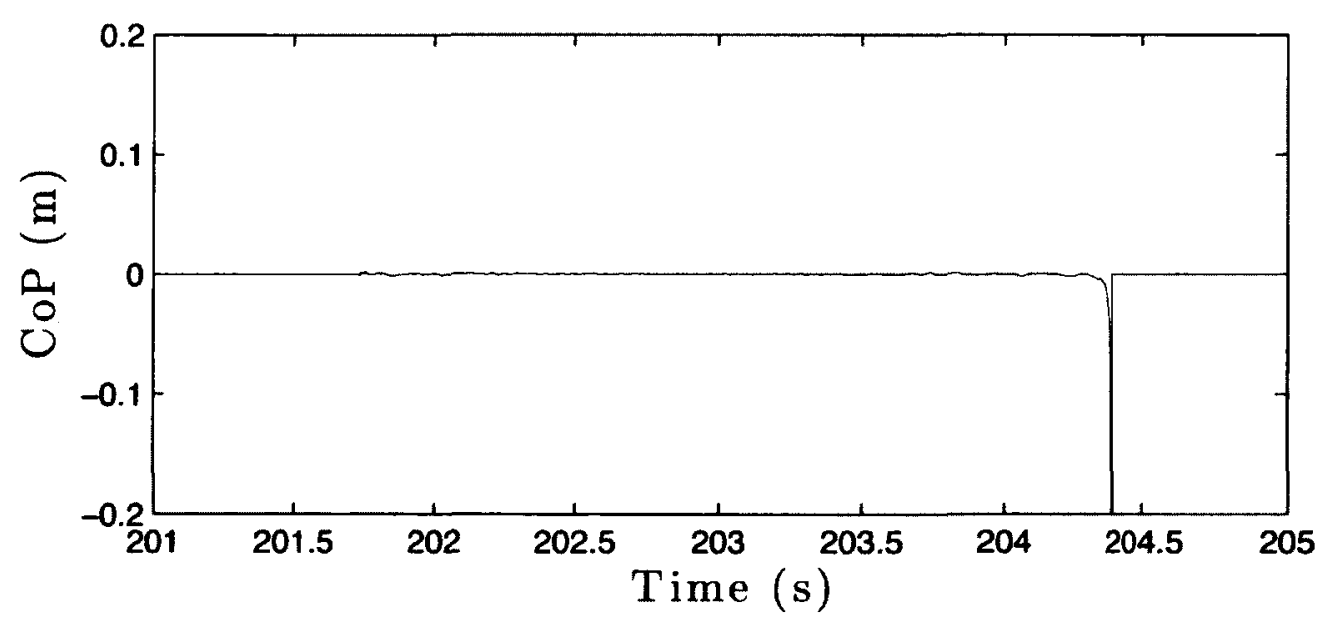

Shape

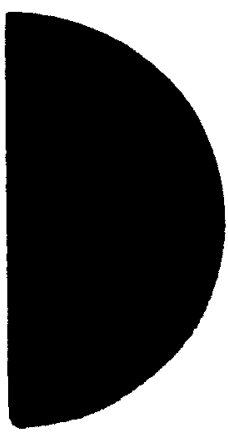

Figure 4.20: Ground Reaction Forces and CoP for a circular virtual tool. $F_{m}$ and $F_{d}$ represent the measured forces at the foot plate and desired forces from the haptic rendering algorithm. 


\section{Discussion:}

Normal Force: As an attempt to remedy some of the issues found using a rectangular virtual tool, the second test involves using a circular virtual tool for ground reaction force reconstruction. Due to the round geometry of the virtual tool, the sharp force bumps generated from the discrete edges in the rectangular virtual tool are not apparent in this test. There are small force peaks at the beginning and end of the stance phase which are caused from heel down and toe off respectively. This virtual tool, however does not follow the double hump pattern since the user is unable to apply forces with their toe or heel.

Centre of Pressure: Although the circular virtual tool provides a smoother ground contact, a big disadvantage is that the CoP during the stance phase does not vary. At any point during the stance phase, the virtual tool slips along the surface of the ground, causing the ground reaction forces to be directed through the origin of the tool. The momentary spike in $\mathrm{CoP}$ at the end of the stance phase is due to a calculation discontinuity where the normal force becomes much smaller than the generated moment. This spike is not indicative of the actual CoP that is felt by the user.

Subject Feedback: When asked about the fidelity of the circular virtual tool, the user felt as if their foot was pivoting around the hub of a wheel since they were not able to directly apply moments to the ground. This virtual tool is similar to a $3-\mathrm{DoF}$ haptic rendering application in which moments are not generated.

\section{Hybrid Virtual Tool}

\section{Results:}

Figure 4.21 shows the ground reaction forces and CoP for the hybrid virtual tool. 

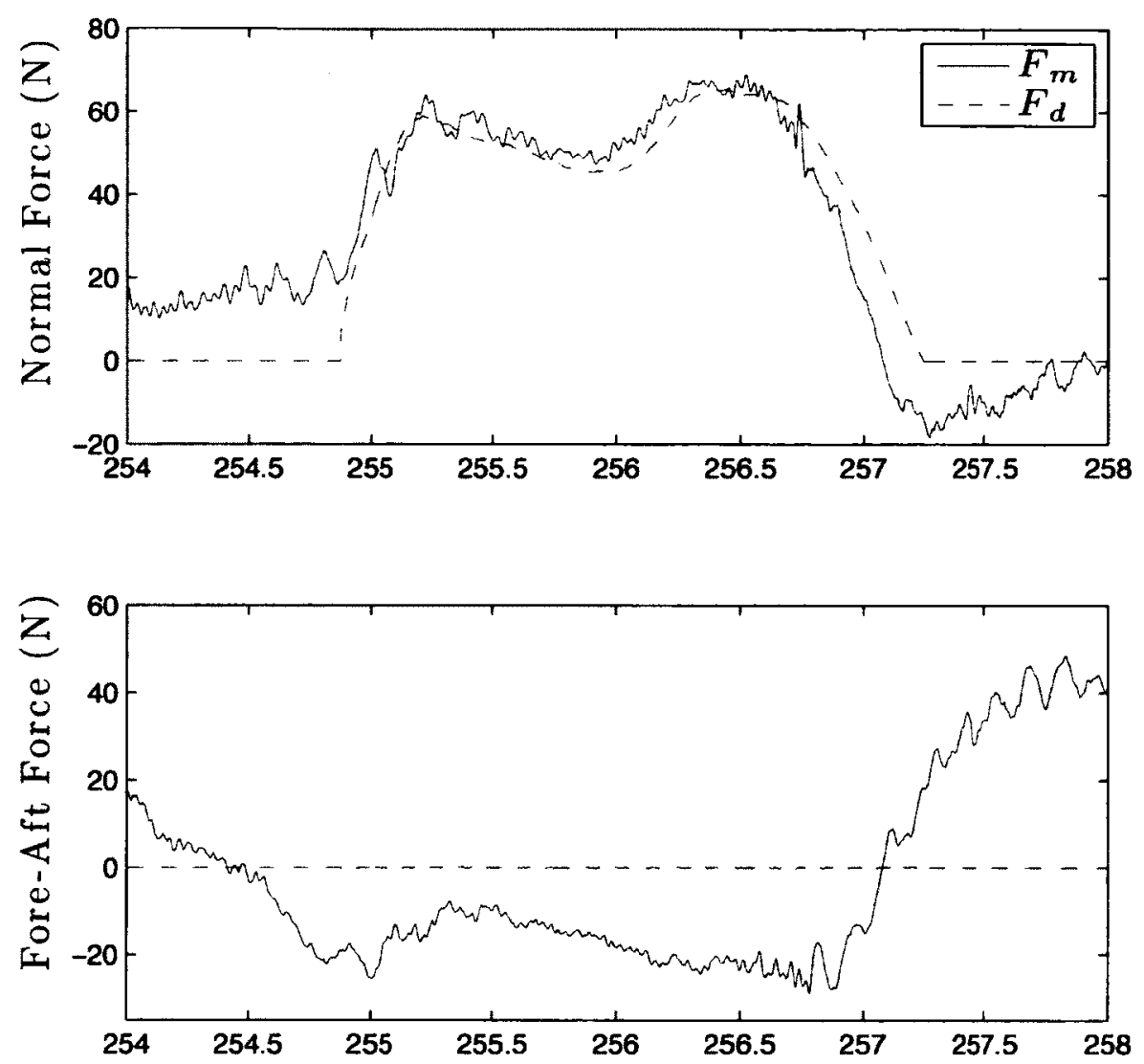

\section{Tool}

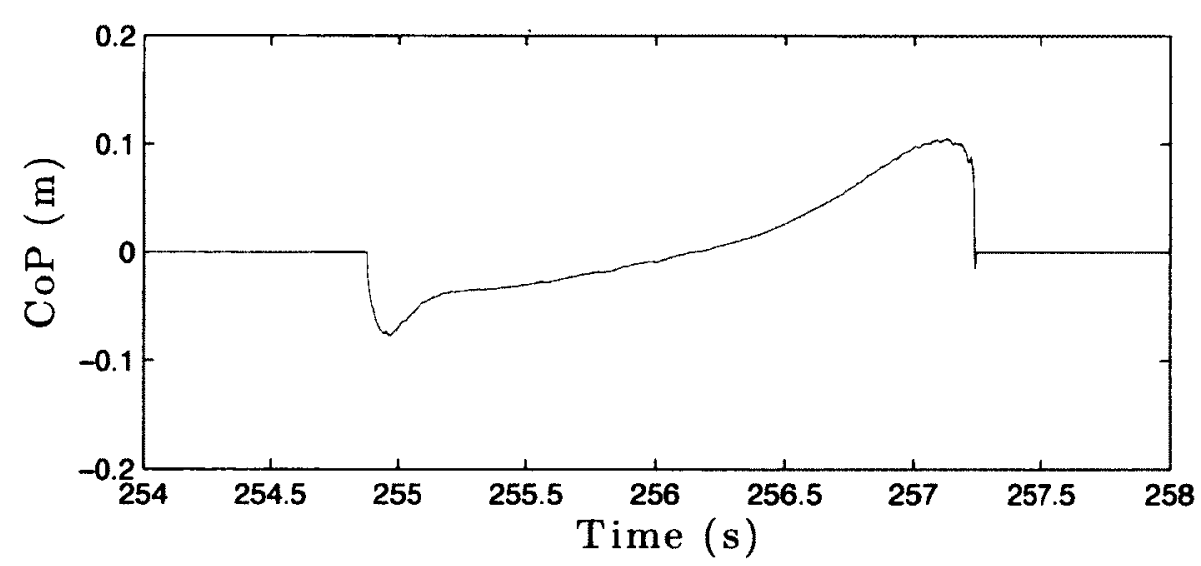
Shape

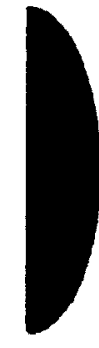

Figure 4.21: Ground Reaction Forces and CoP for a hybrid virtual tool. $F_{m}$ and $F_{d}$ represent the measured forces at the foot plate and desired forces from the haptic rendering algorithm. 


\section{Discussion:}

Normal Force: The hybrid virtual tool was developed to address the issues of both the rectangular and circular geometries. This tool combines the flat bottom of the rectangular tool with the rounded edges of the circular tool to provide smooth transition between sections in the stance phase. The normal force shown in Figure 4.21 follows the characteristic double hump which is seen in the vertical force of Figure 4.3. As stated above, the double hump is caused by a period of weight acceptance at the beginning of the stance phase, and then an acceleration of the body's centre of mass at the end. Although the subject is not applying their full body weight to the foot plate of ViGRR, there is a force peak during heel down which decelerates the foot, and another peak at toe off which accelerates the foot. This force profile however, has no relation to the body's centre of mass as the subject's torso was in a fixed position throughout the experiment.

Based on gait data, the peaks in the vertical ground reaction force should reach around $110 \%$ of the user's body weight, or in our case $636 \mathrm{~N}$. This is substantially higher than the $65 \mathrm{~N}$ peaks which are recorded in Figure 4.21. However, ViGRR was developed as a device to help retrain gait for patient's who are severely immobilized and is not intended to be operated at those loads.

Centre of Pressure: Compared to the rectangular virtual tool, the hybrid yields a smooth transition of CoP from heel to toe. The rounded geometry simulates the ductility of a human shoe, while still allowing a moment to be applied at the toe and heel.

Subject Feedback: Out of the three virtual tools, the user stated that the hybrid geometry best matched the feeling of an actual stance phase.

\subsubsection{Virtual Tool Summary}

The objective of this section was to display a scaled version of the vertical ground reaction force seen during normal walking. It was proven that although ground reaction forces can be generated with all three virtual tool profiles, the most accurate representation of forces seen through normal walking are recreated with the hybrid geometry. This geometry also 
allows for a smooth tracking of the CoP of the foot during the stance phase.

Although the stance phase experiments were conducted for a single subject only, results show that the virtual tool geometry has an impact on the vertical ground reaction forces and CoP. The results from this section, however beg the need for further tests with a variety of tool geometries in order to increase the accuracy of the GRF profile and fidelity of the stance phase.

\subsubsection{Stance Phase General Discussion}

Single Leg Experiments: Simulating walking in ViGRR introduces several limitations on the user which may affect the quality of the exercise. One obvious limitation of the ViGRR prototype is that only a single leg is following the gait trajectory while the other is stationary. Not only is this an unnatural motion for the user, but it also affects the ground reaction force data since there is a period of double stance in which forces are being generated by both feet. Therefore, construction of the second robot arm is essential in order to properly recreate a full gait cycle.

Seat Angle: A result which has not been studied before is the effect of simulated walking while in a seated position. There have been many robotic devices which employ the use of body weight support during the gait cycle, however the patient's torso is always oriented vertically. By changing the orientation of the user, there is now a portion of the gravity vector which is acting against the leg during the swing phase. As future work, research into the effects of varying seat angle, and therefore the gravity vector, on the forces and torques exerted by the user will give insight into this phenomenon.

Motion Constraints: Much like the HapticWalker, the ViGRR prototype constrains the motion of the leg to the $X Y$ plane. This constraint can have adverse long term effects on gait dynamics [57]. It is recommended that an extra rotational degree of freedom (Figure 4.22) be placed at the base of the robot to allow for lateral motion of the leg and pelvis about the $Y$ axis. 


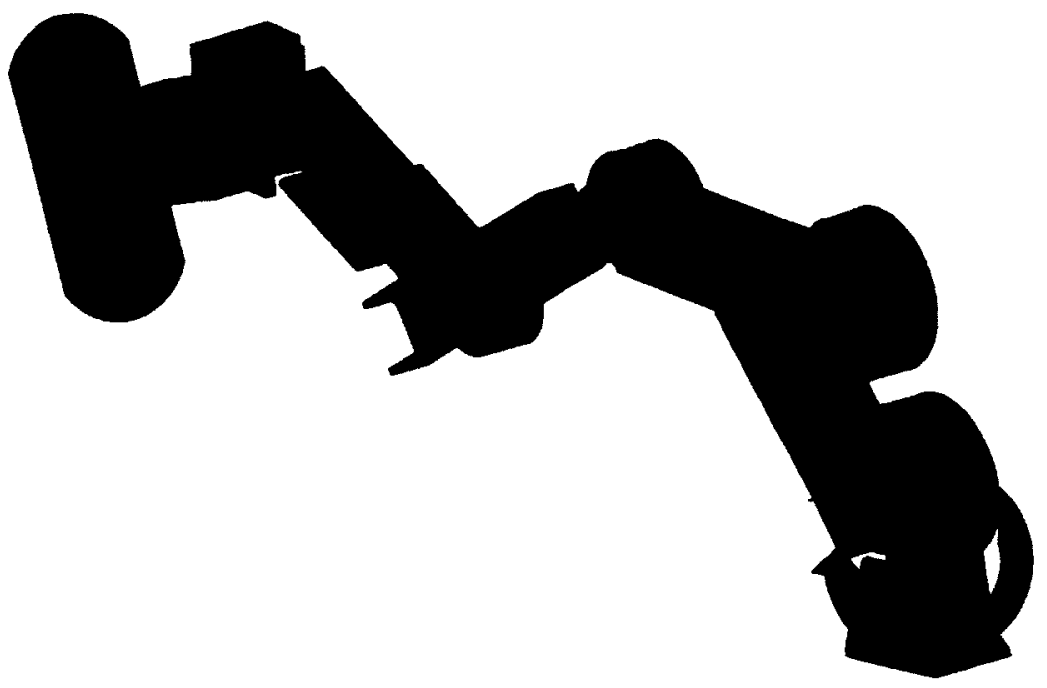

Figure 4.22: Suggested extra rotational degree of freedom about the $Y$ axis.

Heelstrike Transient: The heelstrike transient which appears in most GRF profiles is missing from the stance phase plots shown above. Since a typical heelstrike transient lasts around $10-20 \mathrm{~ms}$, it is unlikely that the FT sensor on ViGRR is unable to measure it [21]. A more plausible suggestion is that the haptic ground stiffness is too soft to generate the momentary force spike.

\subsection{Virtual Reality Exercises}

Several virtual reality exercises have been developed for ViGRR in order to showcase the capabilities of the platform. These exercises are developed with the use of the haptic rendering algorithm in order to provide an engaging and fun experience. The exercises presented below were tested with a healthy subject with the intention of receiving feedback about the level of engagement and value with respect to therapy.

\section{Proposed Applications to Rehabilitation}

As stated in Chapter 1, neural plasticity can only be achieved if the patient is actively engaged in the task they are performing. The virtual reality exercises developed for ViGRR 
are targeted at two main areas for optimizing neural plasticity: engagement and salience. These experiments are developed with the purpose of demonstrating how the ViGRR platform can be used as a rehabilitation tool and are subjective to the author's opinion regarding rehabilitation. Collaboration with occupational therapists is needed to generate exercises which comply with standard rehabilitation practices.

The merit of each exercise will be evaluated based on salience, level of engagement, force input, impairment level of the user, and therapy type. A description of each criteria is listed below.

\section{Salience}

The salience of a given exercise relates to how applicable the therapy is to everyday functions.

\section{Engagement}

Relating to the concept of neural plasticity, the level of engagement of the patient during an exercise is crucial to the quality of their therapy. This criteria determines how engaged the patient is during the exercise.

\section{Force Input}

This criteria relates to how high the force intensity of each exercise can be increased to. A high force input for an exercise can provide large forces to a patient leg whereas a low force input requires a smaller force effort.

\section{Impairment Level}

Some of the exercises that were developed require a minimum level of range of motion and leg strength. For this reason, a maximum impairment level of the patient is chosen for each exercise.

\section{Therapy Type}

Each exercise provides a specific type of therapy which is useful for targeting the needs of a variety of patients. These types include balance, strength, range of motion, and speed. 


\subsubsection{Exercise 1: Leg Press}

The first virtual reality exercise developed for ViGRR is designed to simulate a weighted leg press. The patient will push on a weight by extending their leg in the $X$ direction, while gravity is acting in the opposite direction. This weight, shown as the grey box in Figure 4.23 , can be varied depending on the strength of the given patient. Results from the leg press exercise with a healthy subject can be seen in Figure 4.24 below for a weight of $5 \mathrm{~kg}$ and $10 \mathrm{~kg}$.

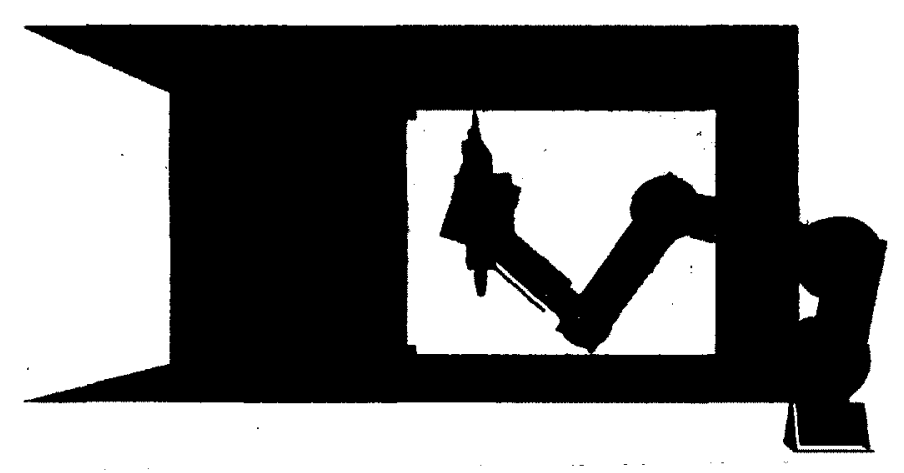

(a)

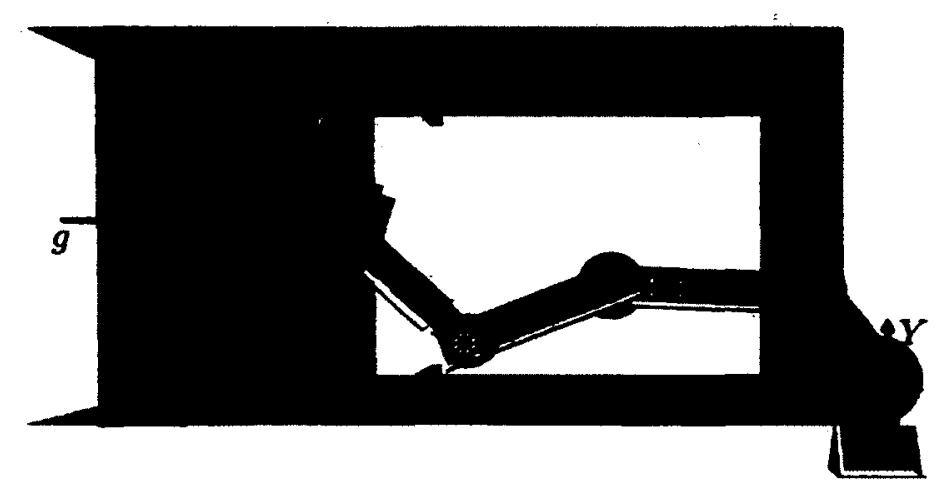

(b)

Figure 4.23: Leg press exercise with ViGRR. (a) The virtual tool and weight are shown as the red and grey boxes respectively. (b) The patient pushes in the $X$ direction, acting against gravity. 

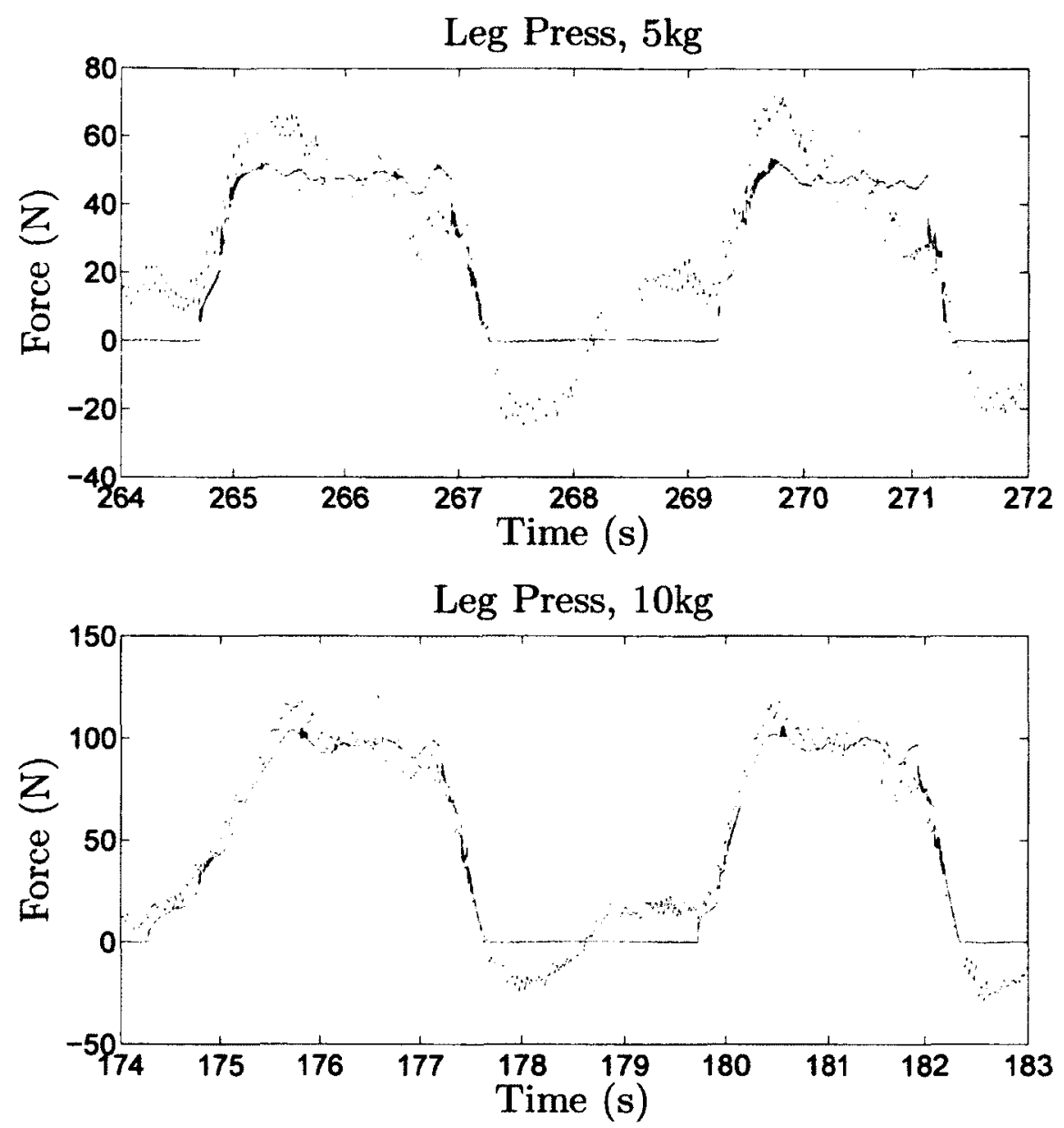

Figure 4.24: Leg Press experiment for a $5 \mathrm{~kg}$ and $10 \mathrm{~kg}$ weight. The blue line represents the measured forces at the FT sensor $\left(F_{m}\right)$ and the red line represents the haptic interaction forces $\left(F_{d}\right)$.

\section{Proposed Applications to Rehabilitation}

This exercise is targeted at strength training for the patient. A weak patient can start with a small load which gradually increases as they progress. In terms of salience, this exercise helps build muscle for sit-to-stand. The level of engagement of this exercise, however is fairly low as there is no particular objective associated with completing the exercise. A summary of the rehabilitation criteria can be seen in Table 4.6 below. 
Table 4.6: Leg Press Exercise Summary.

\begin{tabular}{c|c}
\hline Salience & Sit-to-stand training \\
\hline Engagement & Low \\
\hline Force Level & Low to High \\
\hline Impairment Level & Mild to Severe \\
\hline Therapy Type & Strength \\
\hline \hline
\end{tabular}

\subsubsection{Exercise 2: Brickbreaker}

Figure 4.25 below shows an exercise which involves bouncing a ball on a paddle in order to make all of the coloured bricks disappear. The position and orientation of the paddle is defined by the position and orientation of the foot plate which is controlled by the user. Force and moment plots of a single trial of the brickbreaker exercise can be seen in Figure 4.26. The graphs show that throughout the experiment the subject produced rapid changes in the force and moment exerted at the foot plate. These changes can relate to the rapid response time necessary to prevent from tripping or avoiding obstacles during walking. 


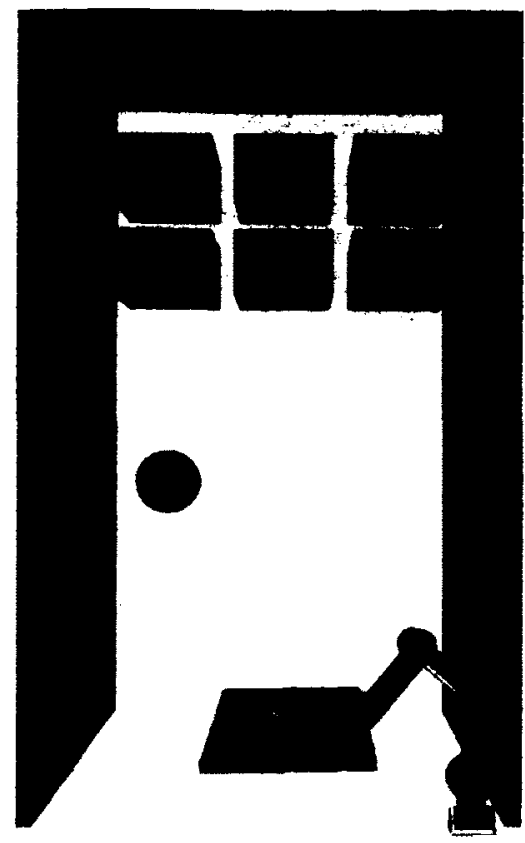

(a)

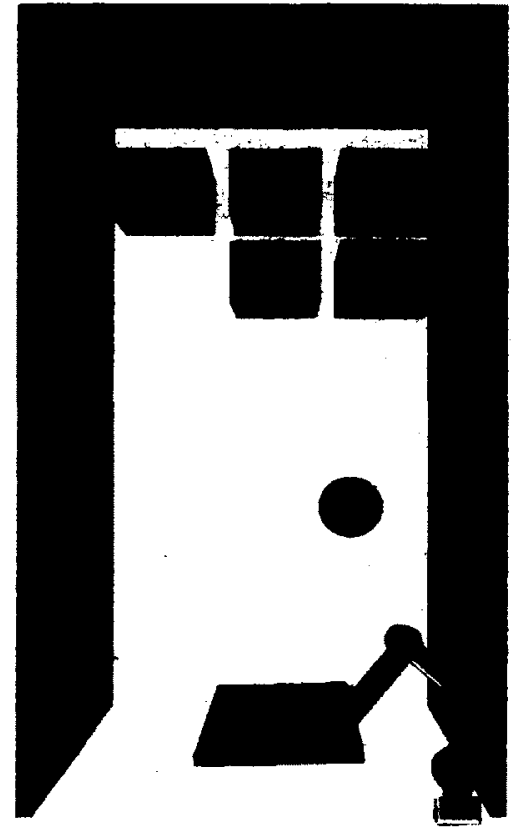

(b)

Figure 4.25: Brickbreaker exercise for ViGRR. (a) Bricks are enabled in the rigid body simulation at startup. (b) Once contacted, the bricks disappear. 

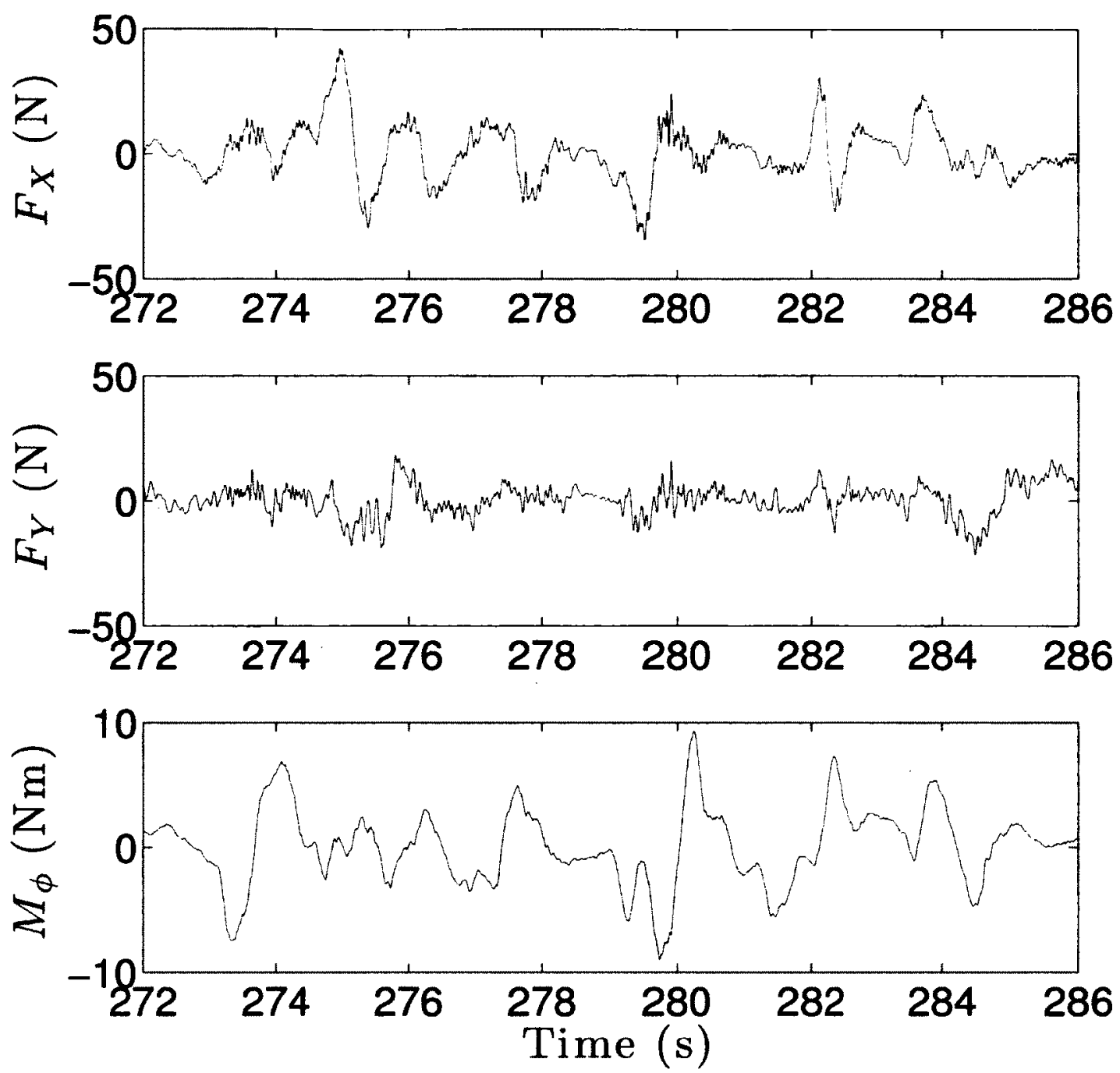

Figure 4.26: Subject interaction loads during the brickbreaker exercise.

\section{Proposed Applications to Rehabilitation}

The main focus of this exercise is to increase the muscle activation speed of the patient. The game initially starts out with a single ball moving at slow speeds. As the patient progresses, difficulty can be increased by adding multiple balls, increasing their speed, and decreasing the size of the paddle. This exercise provides merit in real life applications by training patients for foot placement and obstacle avoidance during the walking. However, 
this exercise could prove too demanding for severely impaired patients if they have a low range of motion. A summary of the rehabilitation criteria can be seen in Table 4.7 below.

Table 4.7: Brickbreaker Exercise Surnmary.

\begin{tabular}{c|c}
\hline \hline Salience & Obstacle Avoidance and Foot Placement \\
\hline Engagement & High \\
\hline Force Input & Low \\
\hline Impairment Level & Mild \\
\hline Therapy Type & Muscle Activation/Speed \\
\hline
\end{tabular}

\subsubsection{Exercise 3: Maze}

This exercise requires the user to navigate a sphere through a maze as seen in Figure 4.27. If the sphere touches a wall, its colour turns red. As with the previous game, the position of the sphere is controlled by the position of the user's foot. The position of the sphere through the maze for one trial can be seen in Figure 4.28 below.

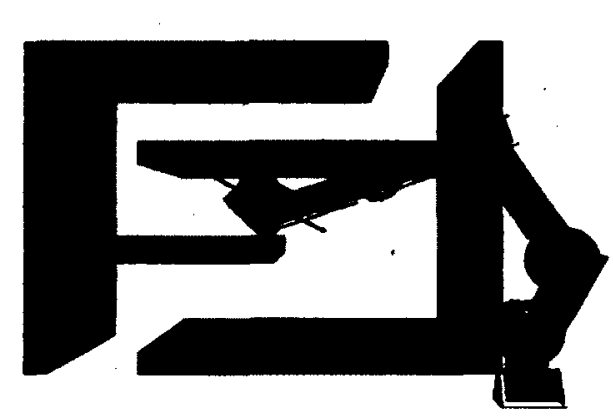

(a)

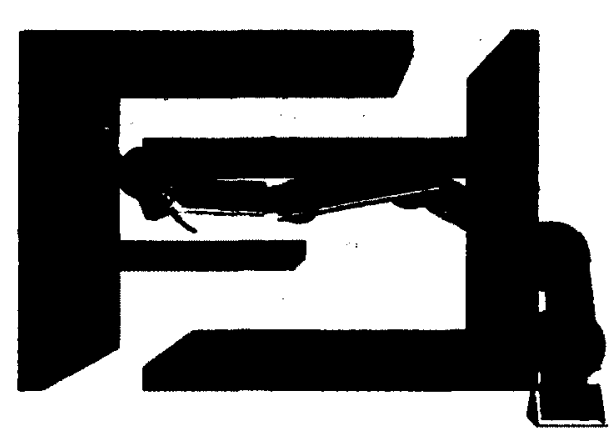

(b)

Figure 4.27: Maze exercise for ViGRR. (a) Virtual tool in free space. (b) Virtual tool in contact with walls. 


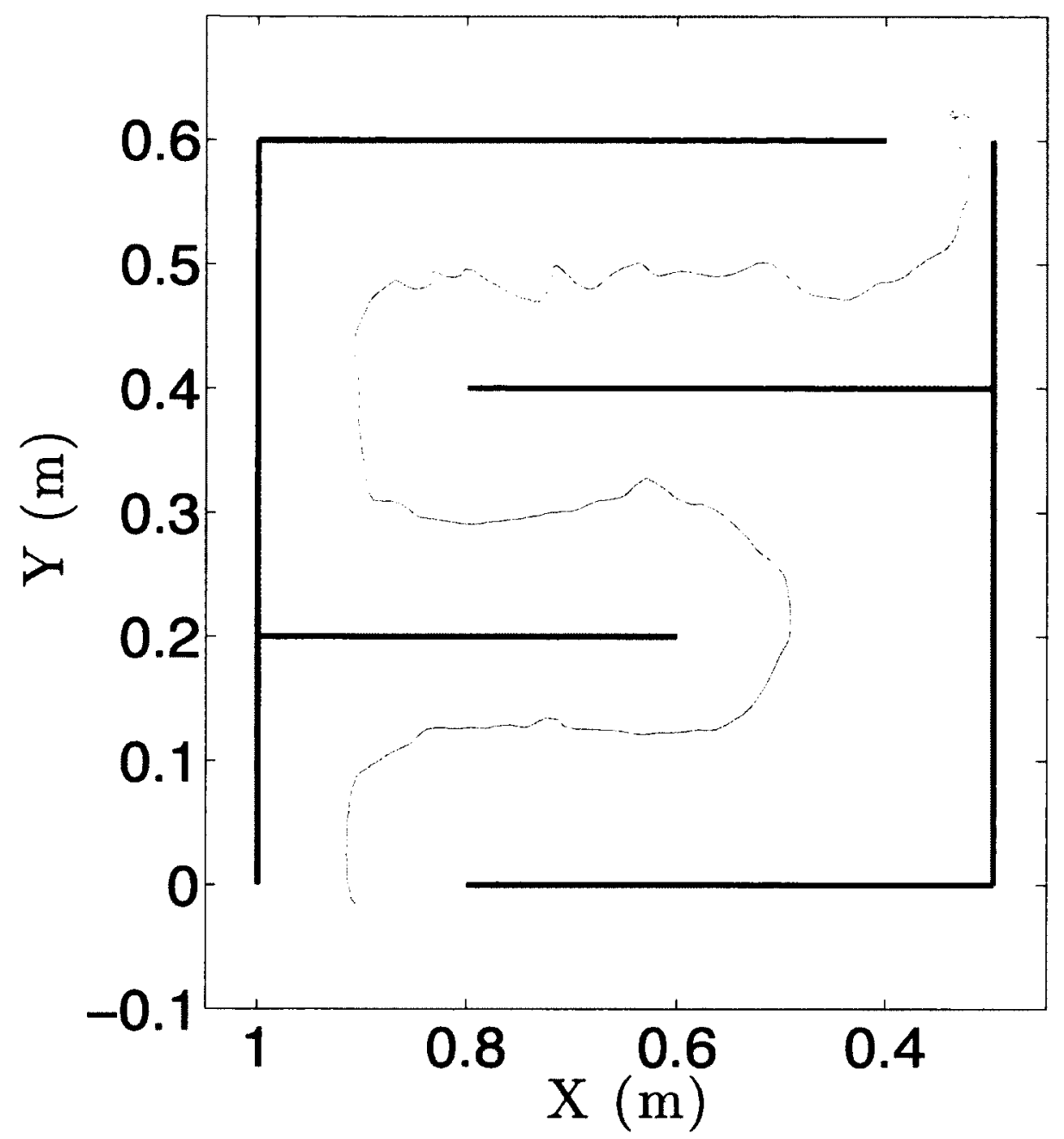

Figure 4.28: Position of the end-effector during the maze exercise. The red lines represent the maze walls and the blue line represents the end-effector position.

\section{Proposed Applications to Rehabilitation}

This exercise focuses on increasing the range of motion of the patient and isolating different muscles. The maze starts simple, with a small sphere and only a few channels to navigate through. Much like the previous exercise, complexity is increased as the patient progresses by increasing the size of the sphere and decreasing the channel width. A summary of the rehabilitation criteria can be seen in Table 4.8 below. 
Table 4.8: Maze Exercise Summary.

\begin{tabular}{c|c}
\hline \hline Salience & Obstacle Avoidance and Foot Placement \\
\hline Engagement & High \\
\hline Force Input & Low \\
\hline Impairment Level & Mild \\
\hline Therapy Type & Range of motion \\
\hline \hline
\end{tabular}

\subsubsection{Exercise 4: Ball Balance}

This final exercise requires the user to balance a ball on a paddle for a specified amount of time as seen in Figure 4.29. If the ball falls off the paddle, the patient must start over. Difficulty can be increased by decreasing the size of the paddle, or increasing the acceleration due to gravity.

Figure 4.30 and 4.31 show the results of the ball balance test with an acceleration due to gravity of $1 \frac{m}{s^{2}}$ and $3 \frac{m}{s^{2}}$ respectively. As can be seen from Figure 4.30, the subject was easily able to balance the ball on the paddle for an extended period of time. However, as the acceleration of gravity increased, the subject could only keep the ball balanced for roughly 4 seconds as seen in Figure 4.31. 


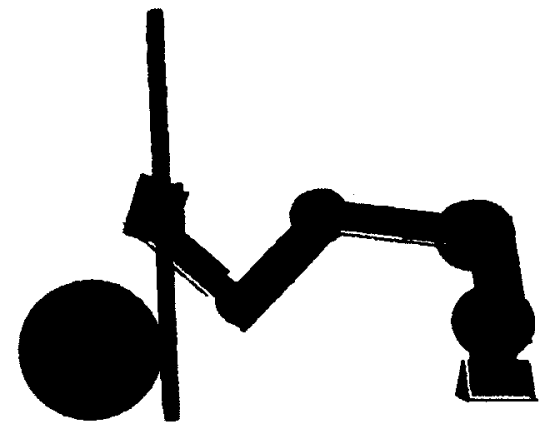

(a)

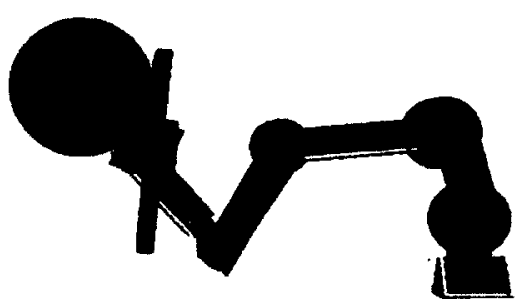

(b)

Figure 4.29: Balance training exercise for ViGRR. (a) The balance block starts large for severely impaired patients. (b) Smaller balance block for increased difficulty. 

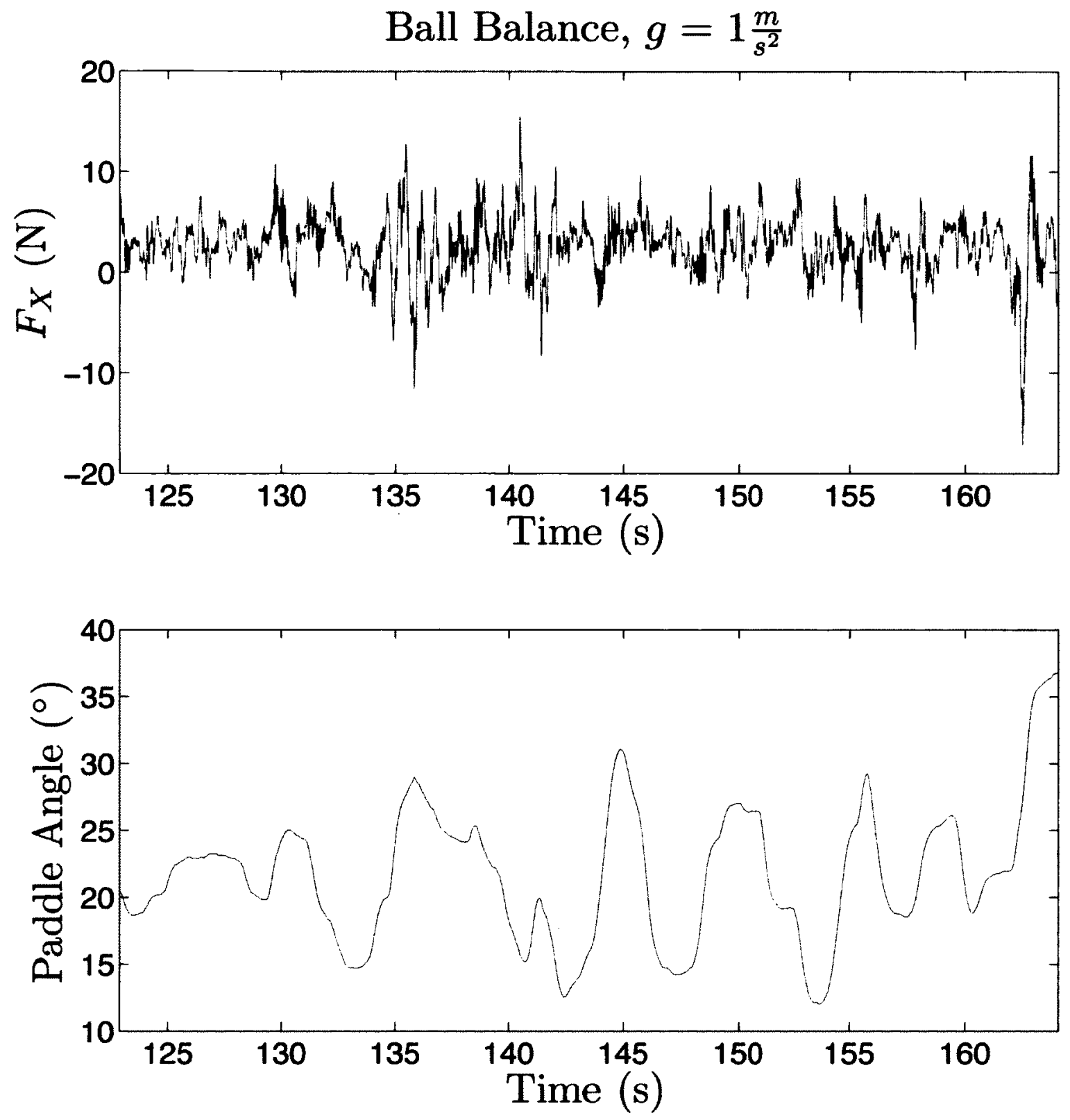

Figure 4.30: Force and paddle angle for the ball balance exercise with $g=1 \frac{m}{s^{2}}$. 

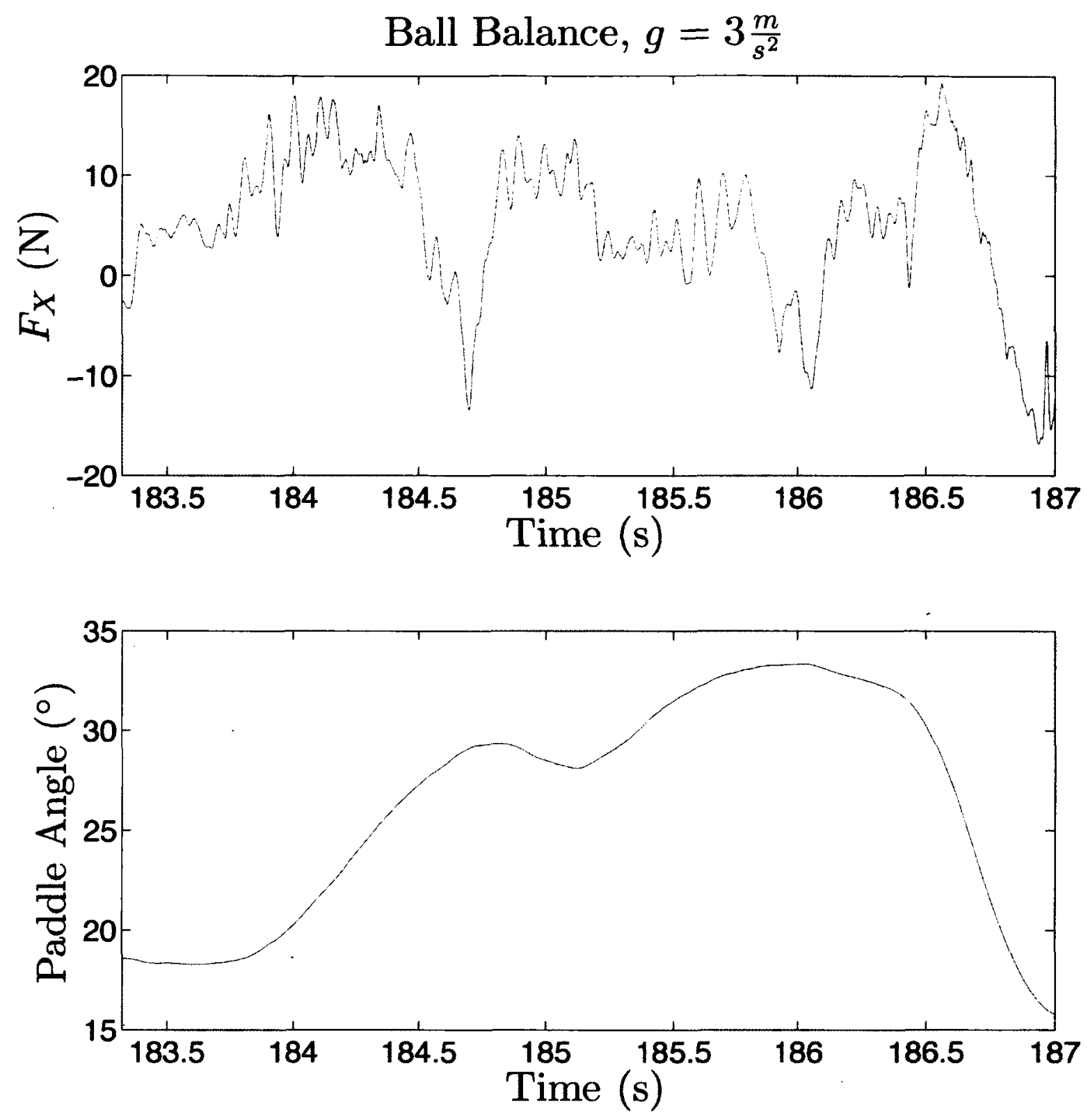

Figure 4.31: Force and paddle angle for the ball balance exercise with $g=1 \frac{m}{s^{2}}$.

\section{Proposed Applications to Rehabilitation}

This final exercise is developed for balance training of patients who have trouble standing or walking on their own. At the moment this exercise provides a moderate level of engagement, however this can be increased by adding a timer to the visualization which encourages the 
patient to improve on their previous score. Once again, this exercise is not intended for severely impaired patients since a fair amount of range of motion is required to complete the task. A summary of the rehabilitation criteria can be seen in Table 4.9 below.

Table 4.9: Ball Balance Exercise Summary.

\begin{tabular}{c|c}
\hline Salience & Standing/Walking \\
\hline Engagement & Moderate \\
\hline Force Input & Low to High \\
\hline Impairment Level & Mild to Moderate \\
\hline Therapy Type & Balance \\
\hline \hline
\end{tabular}

\subsubsection{Summary}

This chapter focused on the development of ViGRR into a lower limb haptic rehabilitation robot. In particular, ViGRR was evaluated on its ability to reproduce the swing and stance phase of a gait cycle. Results show that although portions of the swing phase were tracked with minimal error, the impedance parameters of the robot were too high to allow for a smooth, unhindered motion. As expected, these tracking errors increased with the increase in walking speed. For the stance phase, several virtual foot geometries were introduced in order to determine the best possible solution for recreating the vertical ground reaction force seen through normal, flat-ground walking. Of these profiles, a hybrid virtual tool consisting of a flat bottom with curved ends was chosen as the ideal geometry. The hybrid geometry was capable of producing a moving CoP along the subject's foot during the stance phase. Also, the virtual tool produced a smooth normal force with a characteristic "double hump" seen in most GRF plots. The source of the "double hump" profile seen during normal walking, however, does not appear to be related to the profile obtained through ViGRR since the centre of mass of the subject remained fixed at all times throughout the stance phase.

Virtual reality exercises were then developed and tested in order to highlight the capabilities of ViGRR with respect to rehabilitation. These exercises are focused on providing 
engaging and functional tasks which will benefit both the therapist and patient. After testing with a human subject, the quality of each exercise was evaluated based on salience, level of engagement, force input, patient impairment level, and therapy type. 


\section{Chapter 5}

\section{Conclusions and Future Work}

Rehabilitation robotics is emerging as a critical tool for providing targeted and effective therapy to patients suffering from neurological disorders. Not only does this provide benefit to the patients, but also to the therapists as they are relieved of physical strain while maintaining high level control of the tasks at hand.

There have been many robotic rehabilitation devices developed for the upper extremities. Advancements in the field of lower extremity rehabilitation, however, have been fewer due to the safety concerns associated with the high payloads of the actuators needed for the larger muscle groups. Of the lower extremity devices that have been developed, there is a lack of versatility in the exercises which can be performed. A device which provides only a single exercise can quickly become tiresome and repetitive for a patient.

ViGRR was developed in order to provide a flexible rehabilitation environment for the lower extremities. The large workspace and redundant design makes ViGRR ideal for a wide range of patient sizes and applications.

\subsection{Conclusions}

The realization of ViGRR from concept to prototype was achieved by two previous Master's students from the ABL lab at Carleton University. This initial prototype was developed and tested as a 4-link planar robot, capable of hand controlled force interaction. In order to fully develop ViGRR into a lower limb haptic rehabilitation platform, enhancements in hardware, 
software, and safety were needed. An overview of the completed design enhancements and conclusions drawn from the preliminary lower limb experiments are shown below.

\section{ViGRR Design Enhancements}

The main design enhancements necessary for ViGRR to be safe to conduct human experiments included a stronger base platform, a patient seat, an interface board, upgraded limit switches, a new kinematic calibration, and a detachable foot-robot interface for safe interaction.

The design requirements for the detachable foot-robot interface included complete detachment from the robot, redundant safety mechanisms, variable force safety release, and a lightweight design. The design chosen incorporated electromagnets which can detach if the current in the magnets decrease to zero, or the force applied is greater than the holding force of the magnet and can be seen in Figures 2.20(a) and 2.20(b). The holding force of the interface can also be varied by controlling the amount of current supplied to the electromagnets as seen in Figures 2.22, 2.23, and 2.24.

\section{Haptic Implementation and Verification}

A haptic implementation on ViGRR is essential to providing realistic forces to the user. The requirements for the haptic rendering algorithm were defined with respect to the needs of lower limb fidelity and rehabilitation. These requirements include 6-DoF rendering, friction, variable contact parameters, and real-time QNX compatibility. Several open source haptic rendering solutions were evaluated, however none met all of the requirements listed above. Therefore, a haptic rendering approach was developed with the integration of Open Dynamics Engine. This haptic approach was then implemented on a real-time QNX system and evaluated to ensure the requirements were met as seen in Figures 3.12, 3.14, and 3.16.

\section{Gait Experimentation}

Experiments were conducted with a healthy subject regarding the capability of ViGRR to reproduce the swing and stance phase of a gait cycle. The trajectory tracking swing phase 
experiments can be seen in Figure 4.8, with the corresponding tracking errors in Figures 4.9 and 4.10. These figures show that there is a substantial increase in tracking error during periods of high velocity and acceleration. Feedback from the subject confirms that at low velocity, the tracking of a gait trajectory was easier than at high velocities. The subject also felt a significant amount of muscle fatigue from the high impedance parameters of the controller. From these experiments, it is concluded that the impedance parameters of the controller are too large to obtain a smooth, unhindered motion.

For the stance phase, tests were conducted to determine the optimal virtual tool geometry which will produce contact forces similar to the GRF profile normally seen through walking. The three virtual tools considered were a rectangular geometry, a circular geometry, and a hybrid geometry seen in Figure 4.17. The stance phase experiments for these virtual tools can be seen in Figures 4.19, 4.20, and 4.21. From these tests, it was determined that the hybrid virtual tool best reproduced vertical ground reaction forces normally seen through walking.

\section{Virtual Reality Experimentation}

Virtual reality exercises were developed and tested in order to highlight the capabilities of ViGRR with respect to rehabilitation. These exercises are focused on providing engaging and functional tasks which will benefit both the therapist and patient. Tests with a healthy subject for each exercise can be seen in Figures 4.24, 4.26, 4.28, 4.30, and 4.31. Based on subject feedback, each exercise was evaluated for rehabilitation criteria such as salience, level of engagement, force input, patient impairment level, and therapy type. Tables 4.6, $4.7,4.8$, and 4.9 .

\subsection{Future Work}

The developments presented in this thesis realize ViGRR as a haptic rehabilitation robot for the lower extremity. That being said, there are still some limitations which have been identified throughout this thesis that should be addressed for future iterations of ViGRR. 
First, hardware modifications are presented in future work which will enable ViGRR to be used in clinical studies with patients. Then, software modifications and suggested research directions are proposed for future work.

\section{Hardware Modifications}

The magnetically detachable foot plate designed in Chapter 2 met all of the requirements necessary to function safely for a healthy subject. When the foot plate detaches, the subject must be able to hold the added weight of aluminum. A severely impaired patient, however could be injured due to to this added weight. It is suggested that a second foot plate be designed which will provide less strain on the patient's leg if detached. At the expense of strength, this foot plate could be manufactured out of ABS plastic since a severely impaired patient would not have a high force capacity. For the same purpose as the foot plate, a safety tether should be implemented which prevents the patient's leg from falling if a detachment occurs.

In order to improve upon the fidelity of gait simulation with ViGRR, it is suggested that an extra degree of rotational freedom about the $Y$ axis be implemented at the base of the robot. This will allow for out of plane motion for the user's leg and hip. Caution must be taken in designing the extra degree of freedom as it is now possible for the robot to contact the patient while in the seat. One way to solve this issue would be to install hard limits which physically prevent the robot from rotating into the patient.

Now that a single robot has been developed, implemented, and tested with ViGRR, a second robot for the user's right leg can be designed and manufactured. This robot should take successful elements from the first design, and improve upon the issues associated with the joint and base stiffness.

\section{Control Modifications and Research Directions}

During the gait experiments in Chapter 4, the healthy subject started noticing signs of fatigue. This is partly caused by the relatively high impedance parameters of the admittance controller, and partly due to the effort required by the subject to hold their leg against 
gravity. A gravity compensation scheme which directly cancels the effect of gravity requires an accurate model of the human leg. The human leg is extremely complex and difficult to model, however a suggested gravity compensation method which approximates the human leg as a 2 bar linkage is proposed in Chapter 4 but was not implemented due to time constraints.

The hybrid virtual tool geometry which was tested in Chapter 4, produced a more accurate representation of the vertical ground reaction force compared to the other two geometries. This geometry, however, was chosen with an arbitrary curve at the heel and toe and was not optimized. An optimization of the virtual tool geometry could allow for a higher fidelity of walking.

One direction of potential research for ViGRR is to determine the effects of varying the direction of gravity during walking. This area of research was not possible with other lower extremity platforms, however through ViGRR, this can be achieved by varying the seat angle and virtual ground angle. 


\section{List of References}

[1] O. Lambercy, L. Dovat, R. Gassert, E. Burdet, C. L. Teo, and T. Milner, "A haptic knob for rehabilitation of hand function," IEEE Trans Neural Syst Rehabil Eng, vol. 15, no. 3, pp. 356-366, 2007.

[2] P. Lam, D. Hebert, J. Boger, H. Lacheray, D. Gardner, J. Apkarian, and A. Mihailidis, "A haptic-robotic platform for upper-limb reaching stroke therapy: preliminary design and evaluation results," J Neuroeng Rehabil, vol. 5, p. 15, 2008.

[3] Sensable, "Phantom omni haptic device." Internet.

[4] J. Broeren, M. Rydmark, and K. S. Sunnerhagen, "Virtual reality and haptics as a training device for movement rehabilitation after stroke: a single-case study," Arch Phys Med Rehabil, vol. 85, pp. 1247-1250, Aug 2004.

[5] M. J. Johnson, H. F. M. Van der Loos, C. G. Burgar, P. Shor, and L. J. Leifer, "Experimental results using force-feedback cueing in robot-assisted stroke therapy," IEEE Trans Neural Syst Rehabil Eng, vol. 13, pp. 335-348, Sep 2005.

[6] M. Bouzit, G. Burdea, G. Popescu, and R. Boian, "The rutgers master ii-new design force-feedback glove," IEEE Trans on Mechatronics, vol. 7, no. 2, pp. 256-263, 2002.

[7] VRLOGIC, "Cybergrasp specifications." Internet.

[8] H. Schmidt, "Hapticwalker - a novel haptic device for walking simulation," in Proceedings of EuroHaptics, pp. 60-67, 2004.

[9] S. Hussein, H. Schmidt, S. Hesse, and J. Kruger, "Effect of different training modes on ground reaction forces during robot assisted floor walking and stair climbing," in Proc. IEEE Int. Conf. Rehabilitation Robotics ICORR 2009, pp. 845-850, 2009.

[10] S. Hussein, M. Buchel, and J. Kruger, "Stable, adaptive interaction and contact transition control of a high inertia haptic interface for haptic simulation in gait rehabilitation," in Proc. IEEE Int Robotics and Automation (ICRA) Conf, pp. 4176-4181, 2011. 
[11] M. Girone, G. Burdea, M. Bouzit, and V. Popescu, "A stewart platform-based system for ankle telerehabilitation," Autonomous Robots, vol. 10, pp. 203-212, 2001.

[12] R. F. Boian, J. E. Deutsch, C. S. Lee, G. C. Burdea, and J. Lewis, "Haptic effects for virtual reality-based post-stroke rehabilitation," in Proc. 11th Symp. Haptic Interfaces for Virtual Environment and Teleoperator Systems HAPTICS 2003, pp. 247-253, 2003.

[13] R. F. Boian, M. Bouzit, G. C. Burdea, and J. E. Deutsch, "Dual stewart platform mobility simulator," in Proc. 26th Annual Int. Conf. of the IEEE Engineering in Medicine and Biology Society IEMBS '04, vol. 2, pp. 4848-4851, 2004.

[14] R. Boian, G. Burdea, J. E. Deutsch, and W. S.H., "Street crossing using a virtual environment mobility simulator," in Proceedings of IWVR, 2004.

[15] M. J.-D. Otis, M. Mokhtari, C. du Tremblay, D. Laurendeau, F.-M. De Rainville, and C. M. Gosselin, "Hybrid control with multi-contact interactions for 6dof haptic foot platform on a cable-driven locomotion interface," in Proc. symposium Haptic interfaces for virtual environment and teleoperator systems haptics 2008, pp. 161-168, 2008.

[16] J. Yoon, B. Novandy, C.-H. Yoon, and K.-J. Park, "A 6-dof gait rehabilitation robot with upper and lower limb connections that allows walking velocity updates on various terrains," IEEE Trans on Mechatronics, vol. 15, no. 2, pp. 201-215, 2010.

[17] H. Iwata, H. Yano, and F. Nakaizumi, "Gait master: a versatile locomotion interface for uneven virtual terrain," in Proc. IEEE Virtual Reality, pp. 131-137, 2001.

[18] M. A. Otaduy and M. C. Lin, "A modular haptic rendering algorithm for stable and transparent 6-dof manipulation," IEEE Trans on Robotics, vol. 22, no. 4, pp. 751-762, 2006.

[19] R. Smith, "Ode, open dynamics engine." http://www.ode.org/.

[20] M. W. Whittle, Gait Analysis: An Introduction. Butterworth Heineman Elsevier, 2007.

[21] C. Kirtley, Clinical Gait Analysis: Theory and Practice. Churchill Livingstone Elsevier, 2006.

[22] W. H. Organization, "10 facts on ageing and the life course." Online, September 2011.

[23] Heart and S. F. of Canada, "Stroke statistics." http://www.heartandstroke.com/, 2012.

[24] H. Krebs, L. Dipietro, S. Levy-Tzedek, S. Fasoli, A. Rykman-Berland, J. Zipse, J. Fawcett, J. Stein, H. Poizner, A. Lo, B. Volpe, and N. Hogan, "A paradigm shift for rehabilitation robotics," IEEE Engineering in Medicine and Biology Magazine, vol. 27, no. 4 , pp. $61-70,2008$. 
[25] S. Hesse, H. Schmidt, C. Werner, and A. Bardeleben, "Upper and lower extremity robotic devices for rehabilitation and for studying motor control," Curr Opin Neurol, vol. 16, pp. 705-710, Dec 2003.

[26] J.-M. Belda-Lois, S. Mena-Del Horno, I. Bermejo-Bosch, J. C. Moreno, J. L. Pons, D. Farina, M. Iosa, M. Molinari, F. Tamburella, A. Ramos, A. Caria, T. Solis-Escalante, C. Brunner, and M. Rea, "Rehabilitation of gait after stroke: a review towards a topdown approach," J Neuroeng Rehabil, vol. 8, p. 66, Dec 2011.

[27] H. I. Krebs and N. Hogan, "Therapeutic robotics: A technology push: Stroke rehabilitation is being aided by robots that guide movement of shoulders and elbows, wrists, hands, arms and ankles to significantly improve recovery of patients," Proc IEEE Inst Electr Electron Eng, vol. 94, pp. 1727-1738, Sep 2006.

[28] G. Colombo, M. Jorg, and V. Dietz, "Driven gait orthosis to do locomotor training of paraplegic patients," in Proc. 22nd Annual Int Engineering in Medicine and Biology Society Conf. of the IEEE, vol. 4, pp. 3159-3163, 2000.

[29] R. Rupp, H. Plewa, E. P. Hofer, and M. Knestel, "Motiontherapy@home - a robotic device for automated locomotion therapy at home," in Proc. IEEE Int. Conf. Rehabilitation Robotics ICORR 2009, pp. 395-400, 2009.

[30] R. Boian, A. Sharma, C. Han, A. Merians, G. Burdea, S. Adamovich, M. Recce, M. Tremaine, and H. Poizner, "Virtual reality-based post-stroke hand rehabilitation," Stud Health Technol Inform, vol. 85, pp. 64-70, 2002.

[31] M. McLaughlin, A. Rizzo, Y. Jung, W. Peng, S. Yeh, and W. Zhu, "Haptics-enhanced virtual environments for stroke rehabilitation." 2005.

[32] D. Jack, R. Boian, A. S. Merians, M. Tremaine, G. C. Burdea, S. V. Adamovich, M. Recce, and H. Poizner, "Virtual reality-enhanced stroke rehabilitation," IEEE Trans Neural Syst Rehabil Eng, vol. 9, pp. 308-318, Sep 2001.

[33] L. Marchal-Crespo and D. J. Reinkensmeyer, "Review of control strategies for robotic movement training after neurologic injury," $J$ Neuroeng Rehabil, vol. 6, p. 20, 2009.

[34] A. L. Behrman, M. G. Bowden, and P. M. Nair, "Neuroplasticity after spinal cord injury and training: an emerging paradigm shift in rehabilitation and walking recovery," Phys Ther, vol. 86, pp. 1406-1425, Oct 2006.

[35] L. W. Forrester, L. A. Wheaton, and A. R. Luft, "Exercise-mediated locomotor recovery and lower-limb neuroplasticity after stroke," J Rehabil Res Dev, vol. 45, no. 2, pp. 205$220,2008$.

[36] O. Lambercy, L. Dovat, H. Yun, S. K. Wee, C. W. Kuah, K. S. Chua, R. Gassert, T. E. Milner, C. L. Teo, and E. Burdet, "Effects of a robot-assisted training of grasp 
and pronation/supination in chronic stroke: a pilot study," $J$ Neuroeng Rehabil, vol. 8 , p. 63, Nov 2011.

[37] A. Jarillo-Silva, "Haptic training method for a therapy on upper limb," in Biomedical Engineering and Informatics, pp. 1750-1754, 2010.

[38] H. Schmidt, S. Hesse, and R. Bernhardt, "Safety concept for robotic gait trainers," in Proc. 26th Annual Int. Conf. of the IEEE Engineering in Medicine and Biology Society IEMBS '04, vol. 1, pp. 2703-2706, 2004.

[39] M. J.-D. Otis, S. Perreault, T.-L. Nguyen-Dang, P. Lambert, M. Gouttefarde, D. Laurendeau, and C. Gosselin, "Determination and management of cable interferences between two 6-dof foot platforms in a cable-driven locomotion interface," IEEE Transactions on Systems, Man and Cybernetics, Part A: Systems and Humans, vol. 39, no. 3, pp. 528-544, 2009.

[40] SenseGraphics, "H3d api manual." http://www.h3dapi.org/, September 2009.

[41] L. W. Forrester, L. A. Wheaton, and A. R. Luft, "Exercise-mediated locomotor recovery and lower-limb neuroplasticity after stroke," J Rehabil Res Dev, vol. 45, no. 2, pp. 205$220,2008$.

[42] M. Visintin, H. Barbeau, N. Korner-Bitensky, and N. E. Mayo, "A new approach to retrain gait in stroke patients through body weight support and treadmill stimulation," Stroke, vol. 29, pp. 1122-1128, Jun 1998.

[43] N. D. Neckel, N. Blonien, D. Nichols, and J. Hidler, "Abnormal joint torque patterns exhibited by chronic stroke subjects while walking with a prescribed physiological gait pattern," J Neuroeng Rehabil, vol. 5, pp. 5-19, 2008.

[44] M. Wellner, M. Guidali, J. von Zitzewitz, and R. Riener, "Using a robotic gait orthosis as haptic display - a perception-based optimization approach," in Proc. IEEE 10th Int. Conf. Rehabilitation Robotics ICORR 2007, pp. 81-88, 2007.

[45] J. F. Veneman, R. Kruidhof, E. E. G. Hekman, R. Ekkelenkamp, E. H. F. Van Asseldonk, and H. van der Kooij, "Design and evaluation of the lopes exoskeleton robot for interactive gait rehabilitation," IEEE Trans Neural Syst Rehabil Eng, vol. 15, no. 3, pp. 379-386, 2007.

[46] S. K. Banala, S. H. Kim, S. K. Agrawal, and J. P. Scholz, "Robot assisted gait training with active leg exoskeleton (alex)," IEEE Trans Neural Syst Rehabil Eng, vol. 17, no. 1, pp. 2-8, 2009.

[47] K. Chisholm, "Design and control for a gait rehabilitation robot," Master's thesis, Carleton University, 2010. 
[48] A. Mullins, "Design and implementation of a gait rehabilitation robot manipulator," Master's thesis, Carleton University, 2010.

[49] J. A. Kleim and T. A. Jones, "Principles of experience-dependent neural plasticity: implications for rehabilitation after brain damage.," J Speech Lang Hear Res, vol. 51, pp. S225-S239, Feb 2008.

[50] M. Grunwald, Human Haptic Perception: Basics and Applications. Birkhauser Basel, 2008.

[51] C. B. Zilles and J. K. Salisbury, "A constraint-based god-object method for haptic display," in Proc. IEEE/RSJ Int Intelligent Robots and Systems 95. Human Robot Interaction and Cooperative Robots Conf, vol. 3, pp. 146-151, 1995.

[52] R. E. S. Richard W. Cottle, Jong-Shi Pang, The Linear Complimentary Problem. Society for Industrial and Applied Mathematics (SIAM), 1992.

[53] V. Hayward and O. R. Astley, "Performance measures for haptic interfaces," in In Robotics Research: The 7th Int. Symposium. Giralt, G., Hirzinger, G., Eds., 1996.

[54] B. Siciliano, Robotics: Modelling, Planning and Control. Springer, 2008.

[55] "Iso 9283:1998 manipulating industrial robots - performance criteria and related test methods."

[56] D. A. Winter, The Biomechanics and Motor Control of Human Gait: Normal, Elderly and Pathological. University of Waterloo Press, 1991.

[57] J. F. Veneman, J. Menger, E. H. F. van Asseldonk, F. C. T. van der Helm, and H. van der Kooij, "Fixating the pelvis in the horizontal plane affects gait characteristics," Gait Posture, vol. 28, pp. 157-163, Jul 2008. 


\section{Appendix A}

\section{Ethics Clearance Form}
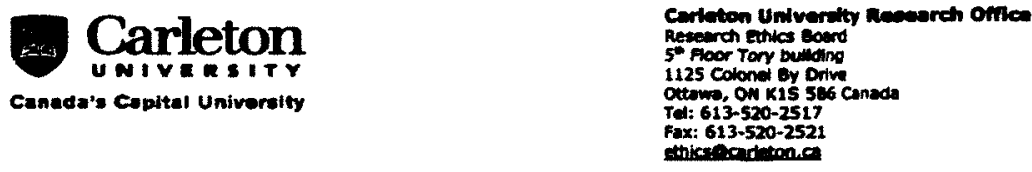

Ethice Clearence form

This is to certify that the Carieton University Research Ethics Board has examined the application for ettical ctearance. The REB found the research project to meet appropriate ethical standards as outlined in the Thi-Council Pollcy Statement: Ethical Conduct for Research Involving Humans, $2^{\text {no }}$ edition and, the Corleton University Policies and Procedures for the Ethical Conduct of Research.

- New charance

$X$ Remewrel of artginal clearence

Original date of cherance: 7 Moy 2010

Date of renewal

Resalecher

Status

11 May 2012

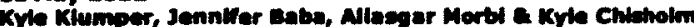

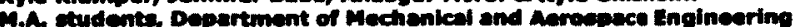

Supervisor

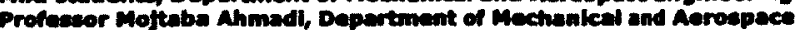
englineoring

Funding status

Project number

Marmunde?

Project

Dealen and Implememtation of the Haptle Gile Rehablitation Robotic

Pletform

Clearance expires: $31 \mathrm{May} 2013$

All researchers are cowerned by the following condtitions:

Anmul status Report: You are required to submit on Amnual Status Report to efther renew dearance or close the file. Faliure to submit the Annual Status Report will reatt in the immedinte suspension of the project. Funded projects will hove sccounts suspended undil the report is submitted and approved.

Chanese to the project: Any changes to the project must be submitted to the Carleton University Resesrch Ethics bound for coprovel. Al changes must be approved prior to the continuance of the research.

adverea mentag Should any participant suffer edverealy from their participation in the project you are required to report the matter to the Carteton Untwersty Research Ethica Board. You must submit a written record of the event and indicate what steps you have taken to resolve the situsetion.

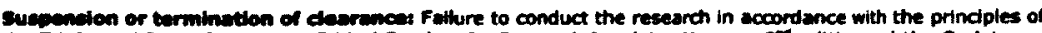
thw Tri-Council Polk Strtement: Ethical Conduct for Reseanch Imvolving Humens $2^{m}$ edition and the Carteton Universty Aollder and Procedures for the Ethical Conduct of Reseyrch may result in the suspention or termination of the research project.

Antonio R. Gualtieri, Chair

Carteton University Research Ethics Board

Figure A.1: Carleton University Ethics Clearance Form 


\section{Appendix B}

\section{Interface Board Diagrams}

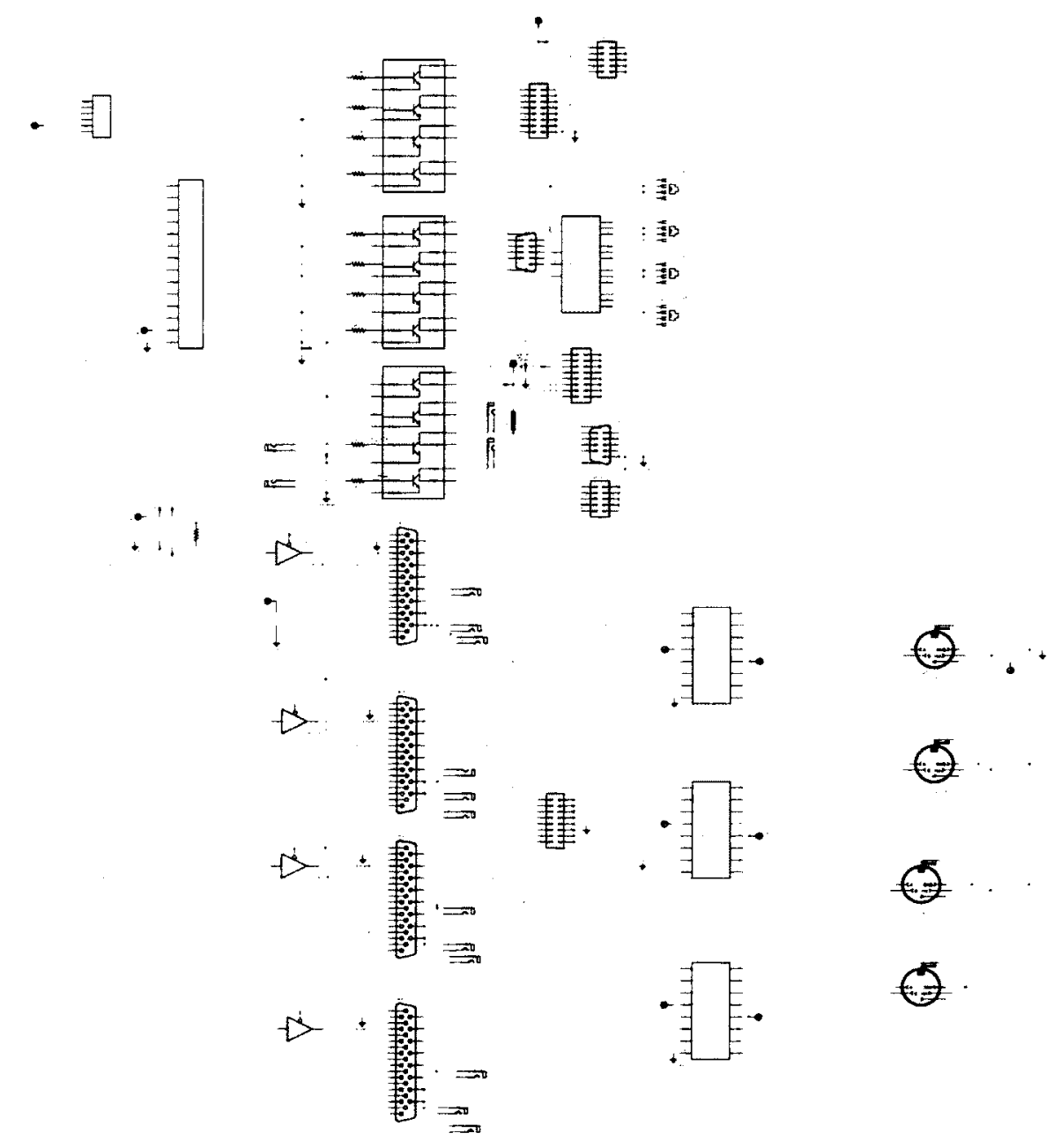

Figure B.1: Interface board schematic diagram 


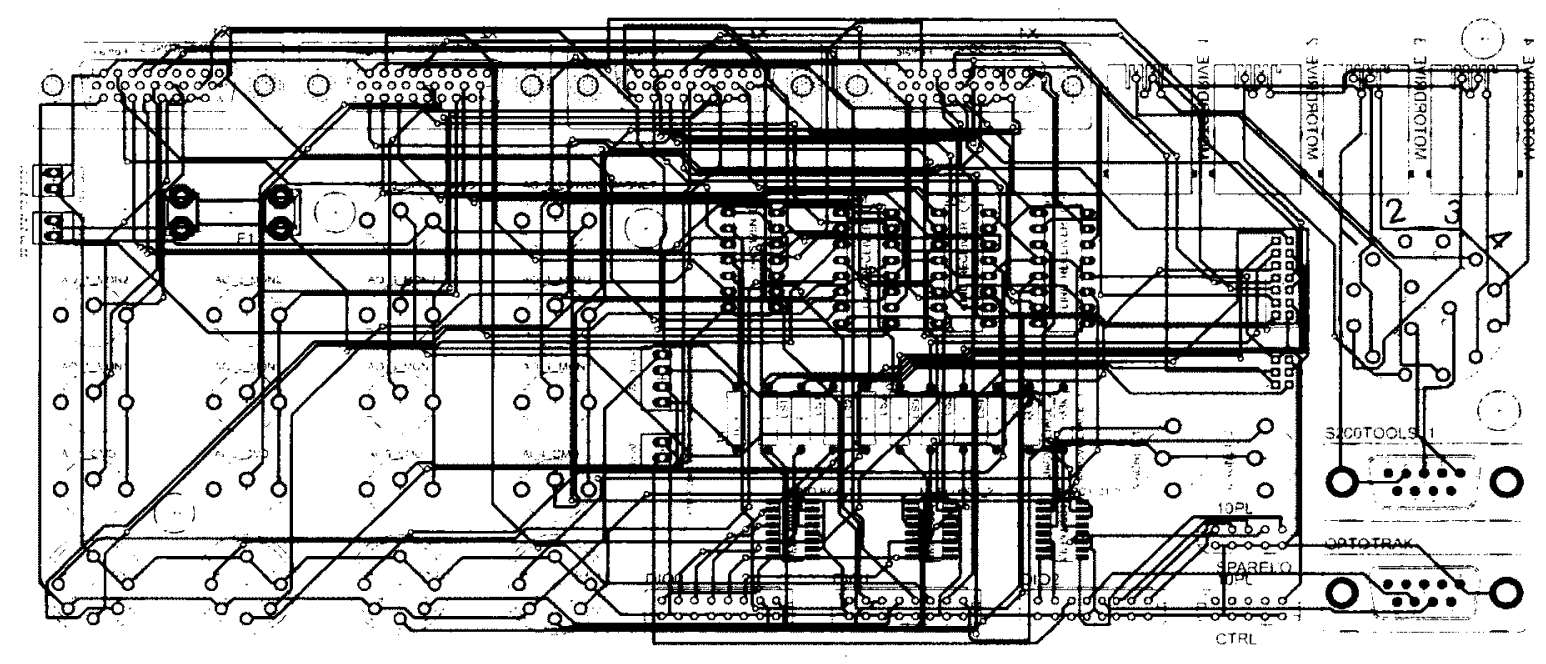

Figure B.2: Interface board pcb view 


\section{Appendix C}

\section{Foot-Robot Coupling}

As stated in Chapter 4, there were oscillations present during the stance phase experiments with a $20^{\circ}$ incline which prevented the user from making a stable contact with the virtual ground. Several possible sources of the oscillations are addressed below, however the root cause was not determined. It is believed that the oscillations are a product of high user loading, robot flexibility and the coupled dynamics of the impedance parameters and human leg, however further biomedical testing is needed to support these claims.

\section{C.1 Contact Parameters}

A first attempt at stabilizing the contact forces between the robot and user was to lower the wall stiffness and damping. Figure C.1 below shows plots of the forces for a similar ground contact with varied stiffness and damping parameters. The graph on the left shows a ground contact with a stiffness of $1500 \frac{\mathrm{N}}{\mathrm{m}}$ and damping of $100 \frac{\mathrm{Ns}}{\mathrm{m}}$, and the graph on the right shows a similar contact with a stiffness of $1000 \frac{\mathrm{N}}{\mathrm{m}}$ and damping of $50 \frac{\mathrm{Ns}}{\mathrm{m}}$. A fourier analysis of the two force plots reveal peaks at $f=6.65 \mathrm{~Hz}$ and can be seen in Figure C.2 below. It was determined that lowering the stiffness and damping constants appeared to have no effect on the oscillatory behavior of the system since the same frequency can be seen for different ground stiffnesses. 

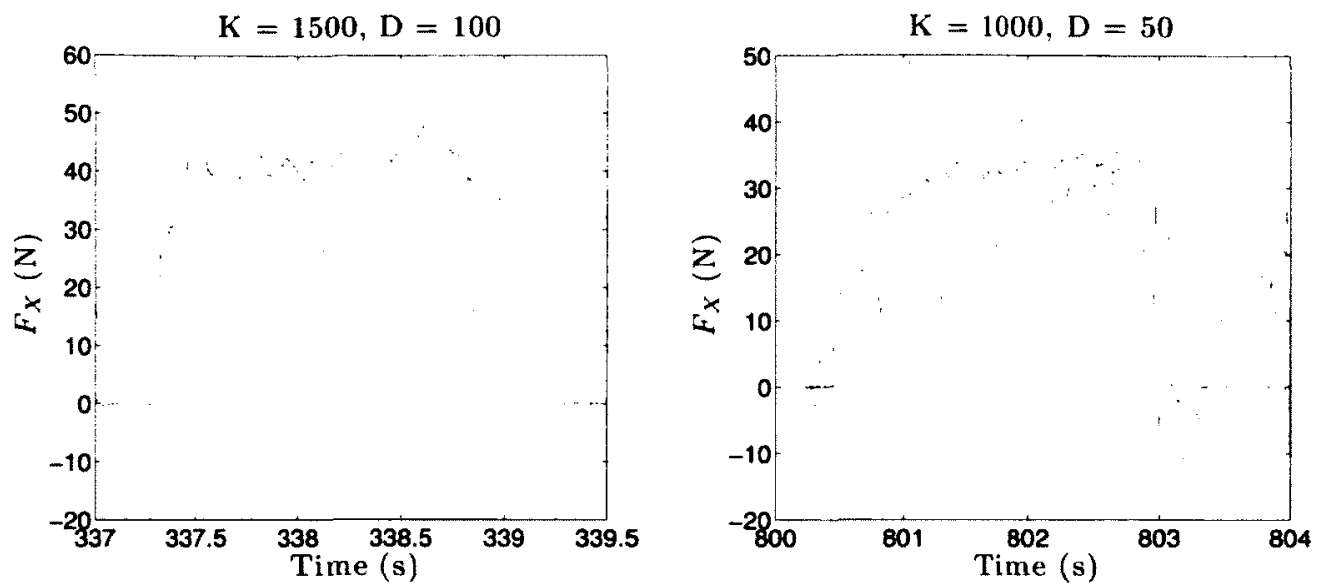

Figure C.1: Haptic interaction force for a subject following a gait trajectory into a virtual ground. The red line show the desired haptic force $F_{d}$ and the blue line represents the measured force $F_{m}$.
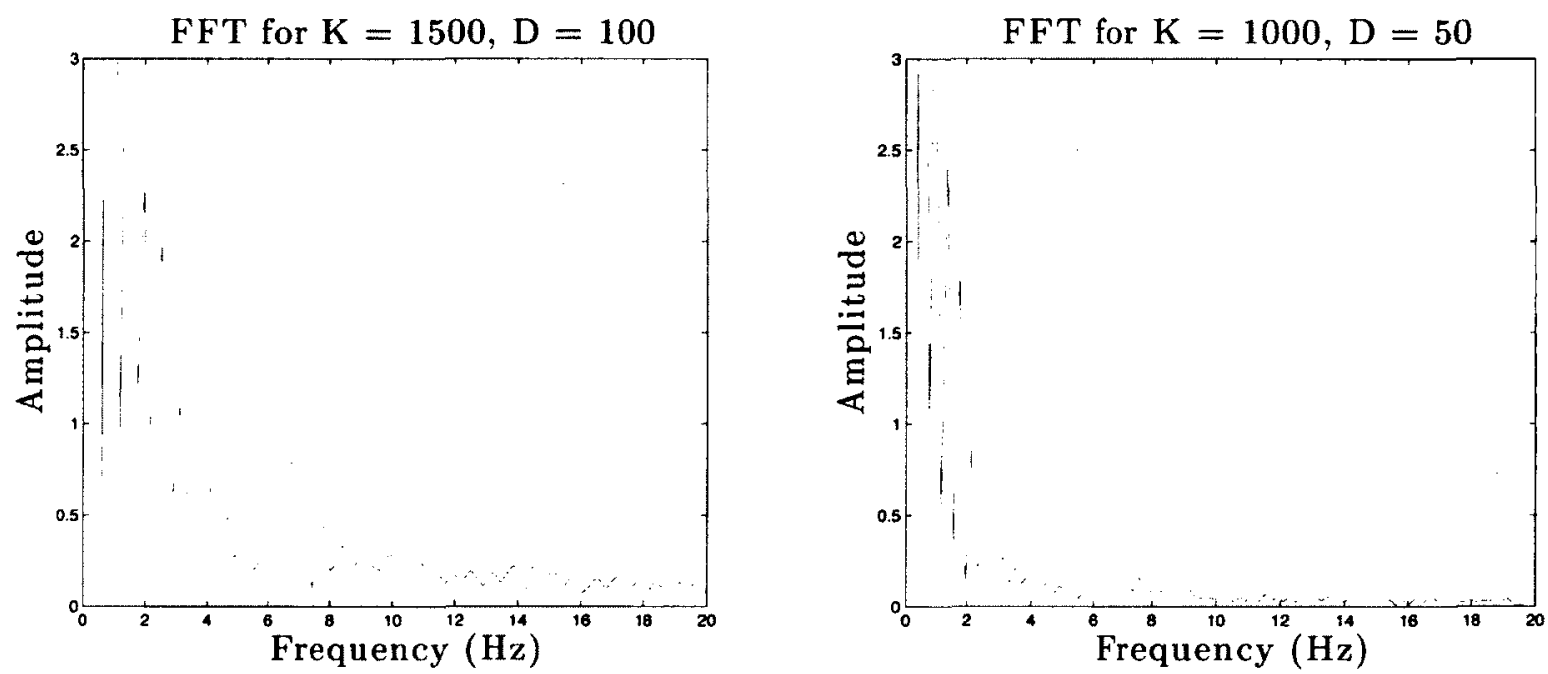

Figure C.2: FFT of the oscillatory haptic interaction forces with varying stiffness and damping parameters. The frequency spike seen in each plot corresponds to $6.65 \mathrm{~Hz}$.

\section{C.2 Robot Flexibility}

The structural design of the robot links allows for rigidity from bending along the direction of the I-beam, however it is susceptible to bending along its major axis. A simple test was conducted which recorded the amount of deflection seen at the robot end-effector in the $Z$ 
direction from a force impulse. The deflection was recorded with the Optotrak measurement system and can be seen in Figure C.3 below. The FFT analysis of the corresponding deflection experiment yielded a structural frequency in the $Z$ direction of $4.12 \mathrm{~Hz}$.
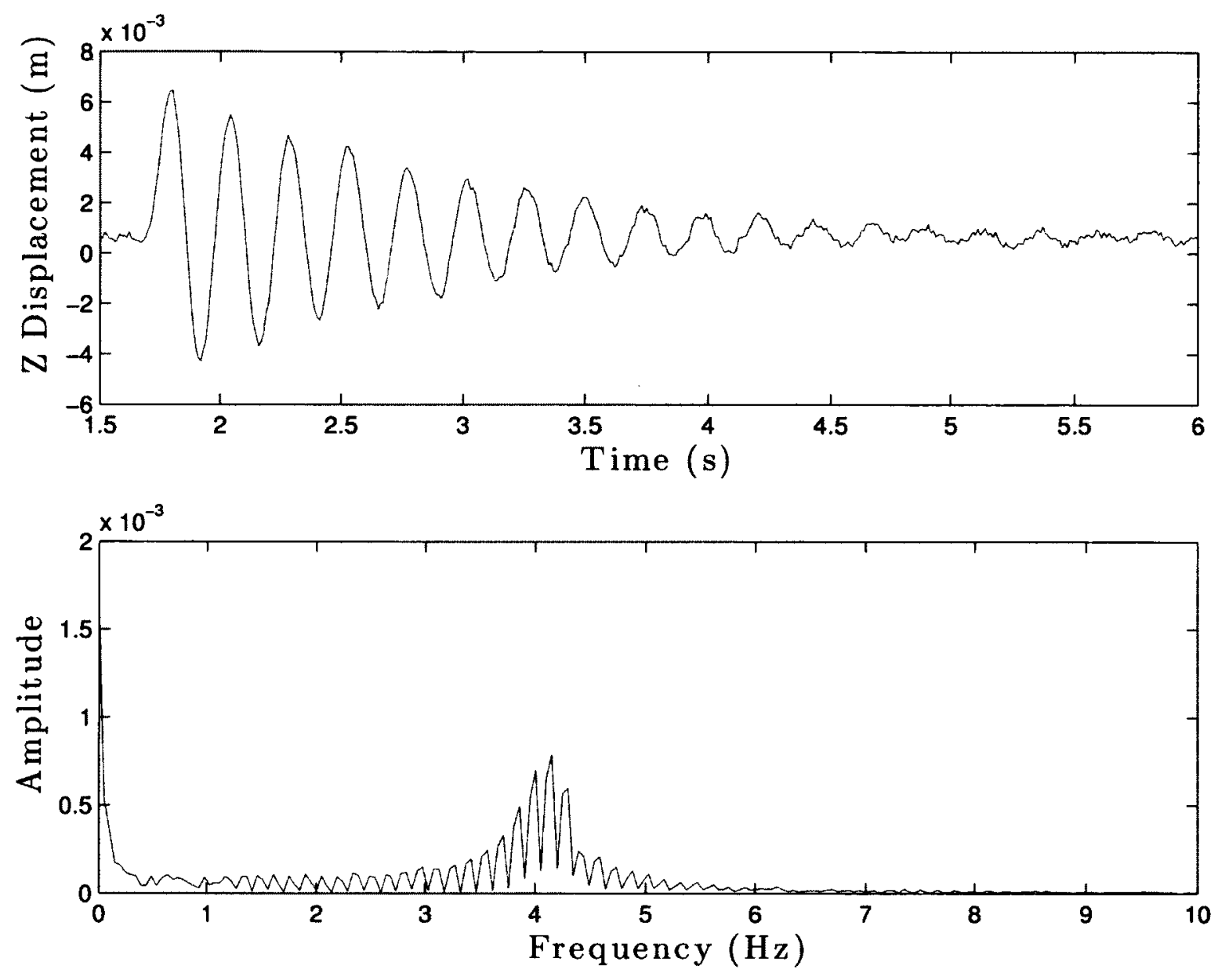

Figure C.3: $Z$ Deflection at the end-effector of the robot and corresponding FFT from a force impulse.

In an attempt to increase the structural frequency of the robot, a steel link was manufactured to replace the aluminum design of link 1 and can be seen in Figure C.4. The deflection experiment was repeated, and a subsequent resonant frequency of $4.3 \mathrm{~Hz}$ was measured as seen in Figure C.5. Although there was a slight increase in the rigidity of the robot, the stiffness associated with link 1 did not seem to affect the overall structural resonance. From the findings obtained, it appears that the overall flexibility of the robot in the $Z$ direction 
is a combination of the flexibility in both the harmonic drives and robot base, however a more in depth study into the robot rigidity is recommended for future work.

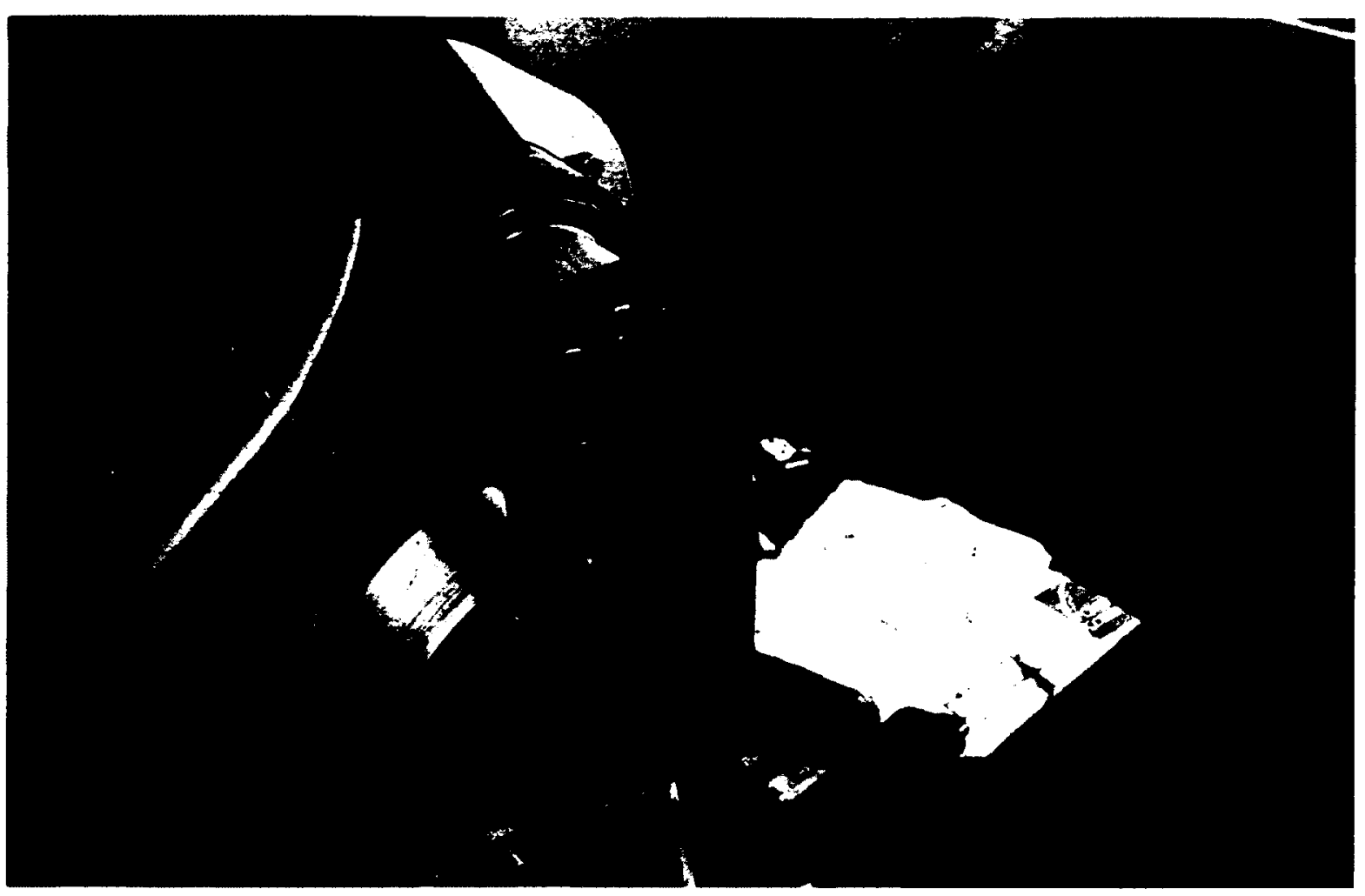

Figure C.4: Steel link replacement for ViGRR link 1. 

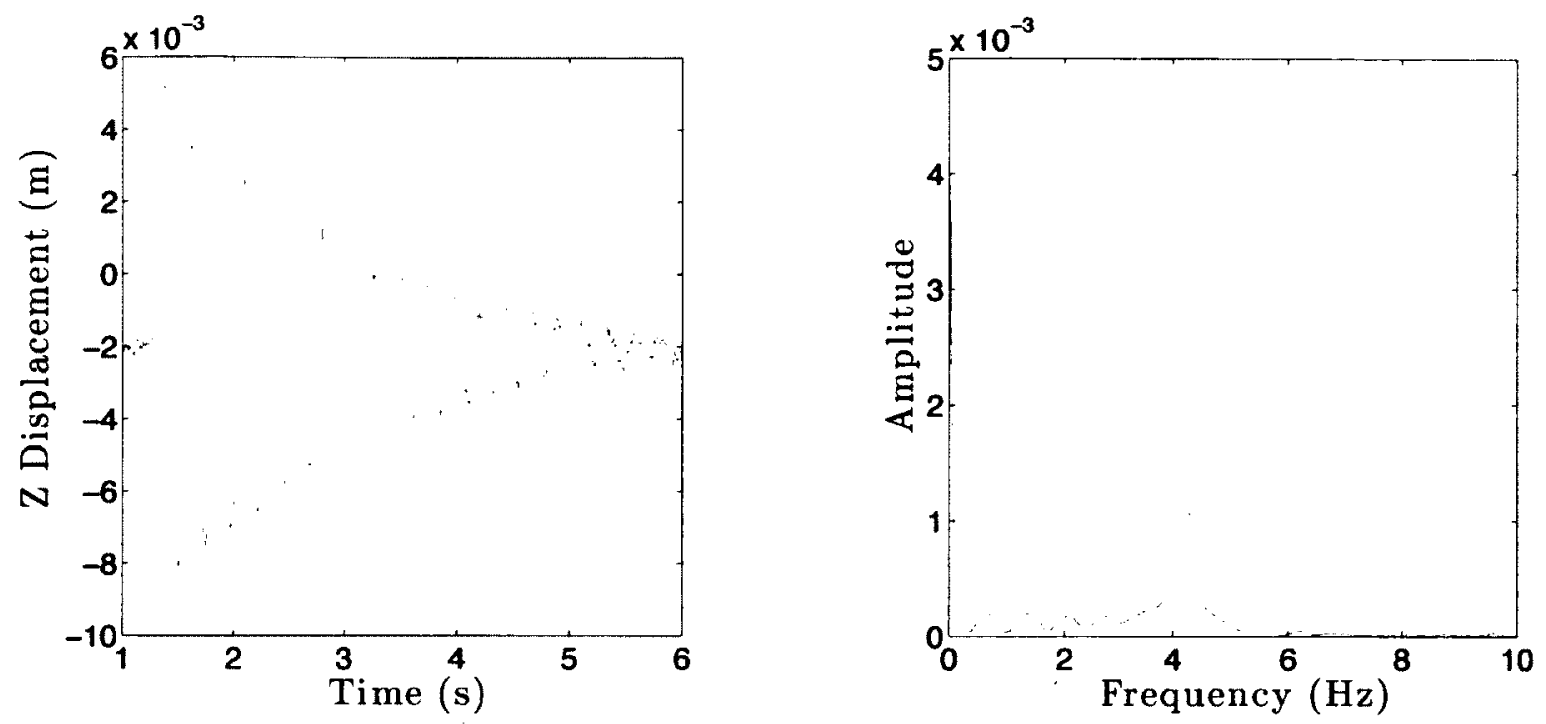

Figure C.5: $Z$ Deflection at the end-effector of the robot and corresponding FFT from a force impulse with a steel first link. 